Supporting Information for

\title{
Ruthenium-Catalyzed Hydroamination of Unactivated Terminal Alkenes with Stoichiometric Amounts of Alkene and an Ammonia Surrogate by Sequential Oxidation and Reduction
}

\author{
Senjie Ma, Christopher K. Hill, Casey L. Olen, John F. Hartwig*
}

Department of Chemistry, University of California, Berkeley, California 94720, United States

\section{Table of Contents}

1. General Information..................................................

2. Procedures for the Synthesis of Ruthenium Complexes......................2

3. Development of Reaction Conditions...................................4

4. Procedures for the Catalytic Hydroamination Reaction.......................4

5. Removal of Pyridyl Group from the Hydroamination Product.................17

6. Determination of the Catalyst Resting State...............................17

7. Kinetic Studies on Catalytic Hydroamination................................19

8. Studies on the Origin of $\mathrm{PEt}_{3}$ Inhibition................................27

9. Deuterium Labelling Experiment.....................................28

10. Catalytic Hydroamination with Acetone as an Additive.....................28

11. Synthesis, Characterization, and Reactivity of the Hydridoruthenium

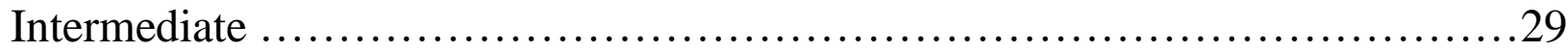

12. DFT Computational Studies.........................................29 
13. X-ray Structures of Ruthenium Complexes..................................48

14. References.......................................................... 53

15. Copies of Spectroscopy data.........................................54

\section{General Information}

All manipulations were performed in a nitrogen-filled glovebox or on a Schlenk manifold unless otherwise noted. Glassware was dried at $150{ }^{\circ} \mathrm{C}$ for at least 4 hours before use. Pentane, $\mathrm{Et}_{2} \mathrm{O}$, THF and hexane were collected from a solvent purification system containing a $0.33 \mathrm{~m}$ column of activated alumina under nitrogen. Diisopropyl ether, 1,2-dichlorobenzene and olefin substrates were degassed and subjected to $4 \AA$ molecular sieves for at least 12 hours prior to use. Anhydrous methanol was purchased from a commercial source, degassed and stored in the glovebox before use. cis-[Ru(DMSO $\left.)_{4} \mathrm{Cl}_{2}\right]^{1}$ and $\left[\mathrm{Ru}_{2}\left(\mathrm{PEt}_{3}\right)_{6}(\mathrm{OTf})_{3}\right](\mathrm{OTf})^{2}$ were prepared following published procedures. All other reagents were purchased from commercial suppliers, stored in the glove box and used as received.

${ }^{1} \mathrm{H},{ }^{13} \mathrm{C},{ }^{19} \mathrm{~F}$ and ${ }^{31} \mathrm{P}$ NMR spectra were recorded on a Bruker 400,500 or $600 \mathrm{MHz}$ spectrometer. ${ }^{1} \mathrm{H}$ chemical shifts are reported in parts per million relative residual protiated solvent as a reference $\left(\mathrm{CHCl}_{3}\right.$ in $\mathrm{CDCl}_{3}: \delta 7.27 \mathrm{ppm} ; \mathrm{CH}_{n} \mathrm{D}_{2-\mathrm{n}}$ in $\left.\mathrm{CDCl}_{2}: \delta 5.32 \mathrm{ppm}\right) .{ }^{13} \mathrm{C}$ chemical shifts are reported in parts per million relative to the deuterated solvent as a reference. ${ }^{31} \mathrm{P}$ chemical shifts were reported in parts per million relative to an $85 \% \mathrm{H}_{3} \mathrm{PO}_{4}$ external standard. ${ }^{19} \mathrm{~F}$ chemical shifts were reported in parts per million relative to an external standard of $\mathrm{CFCl}_{3}$. Elemental analyses were performed at the Microanalytical Facility at the University of California, Berkeley. X-ray crystal structures were obtained at the Small Molecule X-ray Crystallography Facility at the University of California, Berkeley. High-resolution mass spectra were obtained on a highresolution mass spectrometer at the QB3/Chemistry Mass Spectrometry Facility at UC Berkeley and on the Perkin Elmer AxION2 TOF MS operated by the LBNL Catalysis Facility.

\section{Procedures for the Synthesis of Ruthenium Complexes}

cis- $\mathrm{Ru}\left(\mathrm{PMe}_{3}\right)_{4} \mathrm{Cl}_{2}$

cis-[Ru(DMSO) $\left.)_{4} \mathrm{Cl}_{2}\right](0.24 \mathrm{~g}, 0.50 \mathrm{mmol}), \mathrm{PMe}_{3}(1.0 \mathrm{M}$ in toluene, $2.2 \mathrm{~mL}, 2.2 \mathrm{mmol}, 4.4$ equiv), and trifluoroethanol $(2.0 \mathrm{~mL})$ were combined and heated at $100{ }^{\circ} \mathrm{C}$ for 2 hours with stirring. Over this period, all the material dissolved, and the supernatant turned bright orange. The reaction was allowed to cool to room temperature, and the solvent was removed under vacuum. The resulting powder was recrystallized using DCM and ${ }^{i} \operatorname{Pr}_{2} \mathrm{O}$ to yield light yellow crystals. The resulting crystals were rinsed with ${ }^{\mathrm{i}} \mathrm{Pr}_{2} \mathrm{O}(2 \times 5.0 \mathrm{~mL})$ and pentane $(5.0 \mathrm{~mL})$ and dried under high vacuum. Yield $=0.21 \mathrm{~g}(87 \%) .{ }^{1} \mathrm{H}$ NMR $(600 \mathrm{MHz}$, chloroform- $d) \delta 1.56(\mathrm{t}, J=3.2 \mathrm{~Hz}, 36 \mathrm{H})$, 1.47 (m, 36H). ${ }^{31} \mathrm{P}$ NMR (243 MHz, chloroform-d) $\delta$ 20.05. Anal. Calc'd C:30.26 H:7.62 Found $\mathrm{C}: 30.45 \mathrm{H}: 7.84$.

$\left[\mathrm{Ru}_{2}\left(\mathrm{PEt}_{3}\right)_{6} \mathrm{Cl}_{3}\right][\mathrm{Cl}]$ 
cis-[Ru(DMSO) $\left.{ }_{4} \mathrm{Cl}_{2}\right]$ (3.0 g, $\left.6.2 \mathrm{mmol}\right), \mathrm{PEt}_{3}(3.0 \mathrm{~mL}, 20 \mathrm{mmol}, 3.3$ equiv), and $\mathrm{MeOH}(10 \mathrm{~mL})$ were combined and heated at $65^{\circ} \mathrm{C}$ for 90 minutes with stirring. Over this period, all the material dissolved, and the supernatant turned green and then bright orange. The reaction was allowed to cool to room temperature and the solvent was removed under vacuum. The resulting powder was recrystallized using DCM and $\mathrm{Et}_{2} \mathrm{O}$ to yield light yellow crystals. The resulting crystals were rinsed with $\mathrm{Et}_{2} \mathrm{O}(2 \times 10 \mathrm{~mL})$ and pentane $(10 \mathrm{~mL})$ and dried under high vacuum. Yield $=2.9 \mathrm{~g}$ (88\%). ${ }^{1} \mathrm{H}$ NMR (400 MHz, chloroform- $d$ ) $\delta 1.92$ (m, 36H), $1.21(\mathrm{~m}, 54 \mathrm{H}) .{ }^{31} \mathrm{P}$ NMR (162 MHz, chloroform- $d$ ) $\delta$ 34.06. Anal. Calc'd C:41.07 H:8.62 Found C:41.13 H:8.46.

\section{$\left[\mathrm{Ru}_{2}\left(\mathbf{P}^{\mathrm{n}} \mathbf{P r}_{3}\right)_{6} \mathbf{C l}_{3}\right][\mathrm{Cl}]$}

cis-[Ru(DMSO $\left.)_{4} \mathrm{Cl}_{2}\right](0.24 \mathrm{~g}, 0.50 \mathrm{mmol}), \operatorname{PPr}_{3}(0.31 \mathrm{~mL}, 0.25 \mathrm{~g}, 1.6 \mathrm{mmol}, 3.1$ equiv), and a magnetic stir bar were added to a Schlenk flask in a $\mathrm{N}_{2}$ filled glove box. Outside the glove box, degassed $\mathrm{H}_{2} \mathrm{O}(0.80 \mathrm{~mL})$ was added via syringe under nitrogen pressure. The Schlenk flask was sealed and heated at $100{ }^{\circ} \mathrm{C}$ for 2 hours with vigorous stirring. The reaction turned green and the product precipitated as a yellow solid. The reaction was allowed to cool to room temperature, and the yellow precipitation was collected by filtration. The yellow solid was rinsed with $\mathrm{H}_{2} \mathrm{O}$ ( 3 x $5.0 \mathrm{~mL})$ and hexanes $(3 \times 5.0 \mathrm{~mL})$ and dried under high vacuum. Yield $=0.28 \mathrm{~g}(86 \%) .{ }^{1} \mathrm{H}$ NMR (600 MHz, chloroform- $d$ ) $\delta 1.76(\mathrm{~m}, 36 \mathrm{H}), 1.57(\mathrm{~m}, 36 \mathrm{H}), 0.99(\mathrm{t}, J=7.2 \mathrm{~Hz}, 54 \mathrm{H}) .{ }^{31} \mathrm{P}$ NMR (243 MHz, chloroform- $d$ ) $\delta$ 29.16. Anal. Calc'd C:49.69 H:9.73. Found C:49.87 H:9.60.

\section{[Ru2( $\left.\left.\mathrm{PMePh}_{2}\right)_{6} \mathrm{Cl}_{3}\right][\mathrm{Cl}]$}

cis-[Ru(DMSO) $\left.)_{4} \mathrm{Cl}_{2}\right]$ (0.63 g, $\left.1.3 \mathrm{mmol}\right), \mathrm{PPh}_{2} \mathrm{Me}(0.80 \mathrm{~mL}, 0.86 \mathrm{~g}, 4.3 \mathrm{mmol}, 3.3$ equiv), and $\mathrm{MeOH}(1.0 \mathrm{~mL})$ were combined and stirred at room temperature for 90 minutes. The mixture was then placed in the freezer $\left(-30{ }^{\circ} \mathrm{C}\right)$ overnight and the precipitation was collected by filtration, rinsed with $\mathrm{Et}_{2} \mathrm{O}(2 \times 5.0 \mathrm{~mL})$, and dried under vacuum. Combined yield $=0.83 \mathrm{~g}(83 \%) .{ }^{1} \mathrm{H}$ NMR (600 MHz, chloroform- $\left.d_{1}\right) \delta 7.27(\mathrm{t}, \mathrm{J}=7.5 \mathrm{~Hz}, 12 \mathrm{H}), 7.07(\mathrm{~m}, 24 \mathrm{H}), 7.02(\mathrm{~m}, 24 \mathrm{H}), 1.82$ $(\mathrm{m}, 18 \mathrm{H}) .{ }^{13} \mathrm{C}$ NMR $(151 \mathrm{MHz}$, chloroform- $d) \delta 136.70(\mathrm{~m}), 132.93,129.58,127.95,19.88(\mathrm{~m})$. ${ }^{31}$ P NMR (243 MHz, chloroform- $d$ ) $\delta$ 18.93. Anal. Calc'd C:60.63 H:5.09 Found C:60.34 $\mathrm{H}: 5.18$.

\section{cis- $\mathbf{R u}\left(\mathrm{Et}_{2} \mathbf{P}\left(\mathrm{CH}_{2}\right)_{4} \mathbf{P E t}_{2}\right)_{2} \mathbf{C l}_{2}$}

cis-[Ru(DMSO $\left.)_{4} \mathrm{Cl}_{2}\right](0.15 \mathrm{~g}, 0.31 \mathrm{mmol}), \mathrm{Et}_{2} \mathrm{P}\left(\mathrm{CH}_{2}\right)_{4} \mathrm{PEt}_{2}(0.16 \mathrm{~g} 0.68 \mathrm{mmol}, 2.2$ equiv), and $\mathrm{MeOH}(1.0 \mathrm{~mL})$ were combined and heated at $65{ }^{\circ} \mathrm{C}$ for 14 hours with stirring. Over this period, all the material dissolved, and the reaction turned bright red and then orange. The mixture was allowed to cool to room temperature and layered with ${ }^{\mathrm{i}} \mathrm{Pr}_{2} \mathrm{O}(19 \mathrm{~mL})$ to yield yellow crystals. The crystals were collected by filtration, rinsed with ${ }^{i} \operatorname{Pr}_{2} \mathrm{O}(2 \times 5.0 \mathrm{~mL})$ and pentane $(1 \mathrm{~mL})$, and dried under high vacuum. Yield $=0.17 \mathrm{~g}(83 \%) .{ }^{1} \mathrm{H}$ NMR $\left(600 \mathrm{MHz}\right.$, methylene chloride- $\left.d_{2}\right) \delta$ 2.20-2.75 (m, 8H), 1.45-2.05 (m, 20H), 0.95-1.35 (m, 28H). ${ }^{31} \mathrm{P}$ NMR (243 MHz, $\left.\mathrm{CD}_{2} \mathrm{Cl}_{2}\right) \delta$ $32.27(\mathrm{t}, \mathrm{J}=29 \mathrm{~Hz}), 4.62(\mathrm{t}, \mathrm{J}=29 \mathrm{~Hz})$. Anal. Calc'd C:45.00 H:8.81 Found C:45.33 H:9.05.

\section{$\left[\mathbf{R u}_{2}\left(\mathbf{N}\left(\mathrm{CH}_{2} \mathbf{P E t}_{2}\right)_{3}\right)_{2} \mathbf{C l}_{3}\right][\mathbf{C l}]$}

cis-[Ru(DMSO $\left.)_{4} \mathrm{Cl}_{2}\right](0.17 \mathrm{~g}, 0.35 \mathrm{mmol}), \mathrm{N}\left(\mathrm{CH}_{2} \mathrm{PEt}_{2}\right)_{3}(0.11 \mathrm{~g}, 0.35 \mathrm{mmol}, 1.0$ equiv), $\mathrm{MeOH}$ $(1 \mathrm{~mL})$, and a magnetic stir bar were combined and heated at $65^{\circ} \mathrm{C}$ for 5 hours with stirring. The mixture was allowed to cool to room temperature and layered with ${ }^{\mathrm{i}} \operatorname{Pr}_{2} \mathrm{O}(12 \mathrm{~mL})$. The yellow microcrystalline precipitation was collected by filtration, rinsed with ${ }^{\mathrm{i}} \operatorname{Pr}_{2} \mathrm{O}(2 \times 5.0 \mathrm{~mL})$ and dried under high vacuum. Yield $=83 \mathrm{mg}(71 \%) .{ }^{1} \mathrm{H}$ NMR $(600 \mathrm{MHz}$, chloroform- $d) \delta 2.87(\mathrm{~s}$, 
12H), 2.19 (h, $J=9.8,9.1 \mathrm{~Hz}, 12 \mathrm{H}), 1.80$ (dt, $J=15.1,7.5 \mathrm{~Hz}, 12 \mathrm{H}), 1.18$ (p, $J=7.2 \mathrm{~Hz}, 36 \mathrm{H})$.

${ }^{31} \mathrm{P}$ NMR (243 MHz, chloroform- $d$ ) $\delta$ 28.08. Anal. Calc'd C:36.37 H:7.33 N:2.83 Found C:36.53 $\mathrm{H}: 7.19 \mathrm{~N}: 2.64$.

$\operatorname{Ru}\left(\text { PEt }_{3}\right)_{3}\left(\text { NTf }_{2}\right)_{2}(\mathbf{R u}-1)$

$\left[\mathrm{Ru}_{2} \mathrm{Cl}_{3}\left(\mathrm{PEt}_{3}\right)_{6}\right][\mathrm{Cl}](0.38 \mathrm{~g}, 0.36 \mathrm{mmol}), \operatorname{AgNTf}_{2}(0.56 \mathrm{~g}, 1.4 \mathrm{mmol}, 4.0$ equiv) and DCM (5.0 $\mathrm{mL}$ ) were combined and stirred at room temperature for 3 hours. The mixture turned red and orange precipitation was formed during the reaction. The mixture was then filtered, and the solvent was removed under vacuum. The resulting orange solid was recrystallized with DCM and ${ }^{\mathrm{i}} \mathrm{Pr}_{2} \mathrm{O}$ at $-35{ }^{\circ} \mathrm{C}$, washed with ${ }^{\mathrm{i}} \mathrm{Pr}_{2} \mathrm{O}(2 \times 5.0 \mathrm{~mL})$ and dried under high vacuum. Yield $=0.65$ g (89\%). ${ }^{1} \mathrm{H}$ NMR (400 MHz, Methylene Chloride- $\left.\mathrm{d}_{2}\right) \delta 1.91(\mathrm{~m}, 18 \mathrm{H}), 1.24(\mathrm{~m}, 27 \mathrm{H}) .{ }^{31} \mathrm{P}$ NMR $(162 \mathrm{MHz}$, Methylene Chloride-d 2 ) $\delta$ 50.83. Anal. Calc'd C:26.01 H:4.47 N:2.76 Found C:25.91 $\mathrm{H}: 4.49 \mathrm{~N}: 2.70$. The identity of the product was confirmed by x-ray crystallography.

\section{Development of Reaction Conditions}

\section{Preparation of ruthenium complexes for in situ examination of catalytic activity}

The complexes Ru-1 to Ru-6 were generated for in situ for the examination of catalytic activity for convenience. To generate the ruthenium triflimide complex from the corresponding ruthenium chloride precursor in situ, ruthenium chloride complex $(0.020 \mathrm{mmol}$ for dimeric complex or $0.040 \mathrm{mmol}$ for monomeric complex), $\operatorname{AgNTf}_{2}(31 \mathrm{mg}, 0.080 \mathrm{mmol}, 2.0$ equiv relative to $[\mathrm{Ru}])$, trifluoroethanol $(0.50 \mathrm{~mL})$, and a magnetic stir bar were combined in a $1 \mathrm{dram}$ vial. The vial was capped and heated at $80{ }^{\circ} \mathrm{C}$ for $2 \mathrm{~h}$ with stirring and then allowed to cool to room temperature. The solution was filtered, and the volatile materials were evaporated using high vacuum to form a residue. This residue was then triturated with ${ }^{i} \operatorname{Pr}_{2} \mathrm{O}$ and dried. The purity and identity of the ruthenium triflimide complex was verified by NMR spectroscopy.

\section{Conditions for the examination of catalysts for hydroamination of alkenes}

1-dodecene (0.20 mmol, $44 \mu \mathrm{L}, 34 \mathrm{mg}), 2$-amino-5-methylpyridine (0.20 mmol, $22 \mathrm{mg})$, ruthenium complex $(0.010 \mathrm{mmol}, 5.0 \mathrm{~mol} \%[\mathrm{Ru}])$, and solvent $(60 \mu \mathrm{L})$ were combined with a magnetic stir bar in a one-dram vial to form a solution. The vial threads were wrapped with 1 layer of Teflon tape and the resulting mixture was heated at $80{ }^{\circ} \mathrm{C}$ with stirring for 48 hours. The reaction was allowed to cool to room temperature, and yields were determined by ${ }^{1} \mathrm{H}$ NMR spectroscopy using 1,3,5-trimethoxybenzene as the internal standard.

\section{Procedures for the Catalytic Hydroamination Reactions}

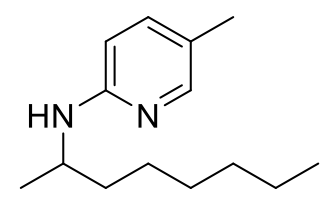

5-methyl- $N$-(octan-2-yl)pyridine-2-amine (2a) 
1-Octene (31 $\mu \mathrm{L}, 23 \mathrm{mg}, 0.20 \mathrm{mmol}), 2$-amino-5-methylpyridine (22 $\mathrm{mg}, 0.20 \mathrm{mmol}$ ), and $\mathrm{Ru}\left(\mathrm{PEt}_{3}\right)_{3}\left(\mathrm{NTf}_{2}\right)_{2}(10 \mathrm{mg}, 0.010 \mathrm{mmol}, 5.0 \mathrm{~mol} \%)$ were combined with $\mathrm{DCB}(60 \mu \mathrm{L})$ and a magnetic stir bar in a one dram vial. The vial threads were wrapped with 1 layer of Teflon tape and the resulting mixture was heated at $80{ }^{\circ} \mathrm{C}$ with stirring for 48 hours. The reaction was allowed to cool, and all of the volatile materials were evaporated. The crude product was purified by silica gel chromatography, eluting with $15 \%$ ethyl acetate in hexanes. Yield $=28 \mathrm{mg}(62 \%)$. ${ }^{1} \mathrm{H}$ NMR $(600 \mathrm{MHz}$, chloroform- $d$ ) $\delta 7.88(\mathrm{~s}, 1 \mathrm{H}), 7.23(\mathrm{dd}, \mathrm{J}=8.4,2.2 \mathrm{~Hz}, 1 \mathrm{H}), 6.28(\mathrm{~d}, \mathrm{~J}=8.4$ $\mathrm{Hz}, 1 \mathrm{H}), 4.16(\mathrm{~d}, \mathrm{~J}=8.1 \mathrm{~Hz}, 1 \mathrm{H}), 3.67(\mathrm{~m}, 1 \mathrm{H}), 2.16(\mathrm{~s}, 3 \mathrm{H}), 1.57-1.24(\mathrm{~m}, 10 \mathrm{H}), 1.17(\mathrm{~d}, \mathrm{~J}=$ $6.4 \mathrm{~Hz}, 3 \mathrm{H}), 0.87(\mathrm{t}, \mathrm{J}=6.9 \mathrm{~Hz}, 3 \mathrm{H}) .{ }^{13} \mathrm{C}$ NMR $(126 \mathrm{MHz}$, chloroform- $d$ ) $\delta 156.8,147.8,138.58$, 121.2, 106.4, 47.5, 37.4, 32.0, 29.5, 26.2, 22.8, 21.1, 17.5, 14.2. GC-MS (EI+): 220 (M), 205 (M$\mathrm{CH}_{3}$ ), 135 ([N-ethyl-5-methylpyridin-2-amine $]^{\circ}$ ).<smiles>Cc1ccc(NC(C)C)nc1</smiles>

\section{5-methyl- $N$-(dodecan-2-yl)pyridine-2-amine (3a)}

1-Dodecene (44 $\mu \mathrm{L}, 34 \mathrm{mg}, 0.20 \mathrm{mmol}$ ), 2-amino-5-methylpyridine (22 $\mathrm{mg}, 0.20 \mathrm{mmol}$ ), and $\mathrm{Ru}\left(\mathrm{PEt}_{3}\right)_{3}\left(\mathrm{NTf}_{2}\right)_{2}(10 \mathrm{mg}, 0.010 \mathrm{mmol}, 5.0 \mathrm{~mol} \%)$ were combined with DCB $(60 \mu \mathrm{L})$ and a magnetic stir bar in a one dram vial. The vial threads were wrapped with 1 layer of Teflon tape and the resulting mixture was heated at $80{ }^{\circ} \mathrm{C}$ with stirring for 48 hours. The reaction was allowed to cool, and all of the volatile materials were evaporated. The crude product was purified by silica gel chromatography, eluting with $15 \%$ ethyl acetate in hexanes. Yield $=35 \mathrm{mg}(63 \%)$. ${ }^{1} \mathrm{H}$ NMR $(600 \mathrm{MHz}$, chloroform- $d) \delta 7.87(\mathrm{~m}, 1 \mathrm{H}), 7.21(\mathrm{dd}, J=8.5,2.4 \mathrm{~Hz}, 1 \mathrm{H}), 6.27(\mathrm{~d}, J=$ $8.4 \mathrm{~Hz}, 1 \mathrm{H}), 4.21(\mathrm{~d}, J=8.6 \mathrm{~Hz}, 1 \mathrm{H}), 3.66(\mathrm{dh}, J=8.5,6.4 \mathrm{~Hz}, 1 \mathrm{H}), 2.14(\mathrm{~s}, 3 \mathrm{H}), 1.51$ (dddd, $J=$ $12.9,10.0,6.5,5.2 \mathrm{~Hz}, 1 \mathrm{H}), 1.44(\mathrm{ddt}, J=12.9,9.5,6.0 \mathrm{~Hz}, 1 \mathrm{H}), 1.39-1.31(\mathrm{~m}, 2 \mathrm{H}), 1.30-$ $1.22(\mathrm{~m}, 14 \mathrm{H}), 1.16(\mathrm{~d}, J=6.4 \mathrm{~Hz}, 3 \mathrm{H}), 0.87(\mathrm{t}, J=7.0 \mathrm{~Hz}, 3 \mathrm{H}) .{ }^{13} \mathrm{C} \mathrm{NMR}(151 \mathrm{MHz}$, chloroform- $d$ ) $\delta 156.8,147.9,138.4,138.4,121.0,106.3,47.4,37.4,32.0,29.8,29.7,29.4,26.2$,

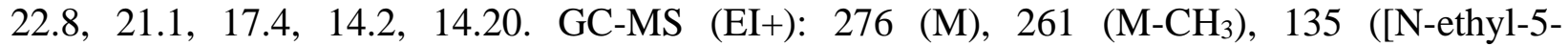
methylpyridin-2-amine] ${ }^{\circ}$.

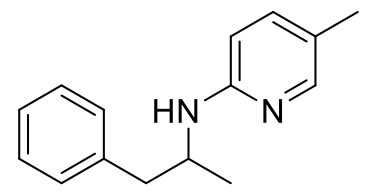

\section{5-methyl- $N$-(1-phenylpropan-2-yl)pyridine-2-amine (4a)}

Allylbenzene (27 $\mu \mathrm{L}, 24 \mathrm{mg}, 0.20 \mathrm{mmol}), 2$-amino-5-methylpyridine (22 $\mathrm{mg}, 0.20 \mathrm{mmol})$, and $\mathrm{Ru}\left(\mathrm{PEt}_{3}\right)_{3}\left(\mathrm{NTf}_{2}\right)_{2}(10 \mathrm{mg}, 0.010 \mathrm{mmol}, 5.0 \mathrm{~mol} \%)$ were combined with DCB $(60 \mu \mathrm{L})$ and a magnetic stir bar in a one dram vial. The vial threads were wrapped with 1 layer of Teflon tape and the resulting mixture was heated at $80{ }^{\circ} \mathrm{C}$ with stirring for 48 hours. The reaction was allowed to cool, and all of the volatile materials were evaporated. The crude product was purified by silica gel chromatography, eluting with $15 \%$ ethyl acetate in hexanes. Yield $=30 \mathrm{mg}(66 \%)$. ${ }^{1} \mathrm{H}$ NMR $(600 \mathrm{MHz}$, chloroform- $d) \delta 7.92(\mathrm{~s}, 1 \mathrm{H}), 7.29(\mathrm{t}, J=7.5 \mathrm{~Hz}, 2 \mathrm{H}), 7.24(\mathrm{dd}, J=8.4,2.4$ $\mathrm{Hz}, 1 \mathrm{H}), 7.23-7.17(\mathrm{~m}, 3 \mathrm{H}), 6.32(\mathrm{~d}, J=8.4 \mathrm{~Hz}, 1 \mathrm{H}), 4.24(\mathrm{~d}, J=8.6 \mathrm{~Hz}, 1 \mathrm{H}), 4.03(\mathrm{dq}, J=8.7$, $6.7 \mathrm{~Hz}, 1 \mathrm{H}), 2.92(\mathrm{dd}, J=13.4,5.1 \mathrm{~Hz}, 1 \mathrm{H}), 2.75(\mathrm{dd}, J=13.4,7.1 \mathrm{~Hz}, 1 \mathrm{H}), 2.17(\mathrm{~s}, 3 \mathrm{H}), 1.16(\mathrm{~d}$, $J=6.5 \mathrm{~Hz}, 3 \mathrm{H}) .{ }^{13} \mathrm{C}$ NMR $(151 \mathrm{MHz}$, chloroform- $d$ ) $\delta 156.4,148.1,138.7,138.5,129.7,128.4$, 
126.4, 121.6, 106.9, 48.3, 42.7, 20.3, 17.5. GC-MS (EI+): 226 (M), $211\left(\mathrm{M}-\mathrm{CH}_{3}\right), 135$ ([N-ethyl5-methylpyridin-2-amine]").<smiles>COc1ccc(CC(C)Nc2ccc(C)cn2)cc1OC</smiles>

$N$-(1-(3,4-dimethoxyphenyl)propan-2-yl)-5-methylpyridin-2-amine (5a)

3,4-Dimethoxy-allylbenzene (36 mg, $0.20 \mathrm{mmol}$ ), 2-amino-5-methylpyridine (22 $\mathrm{mg}, 0.20$ $\mathrm{mmol})$, and $\mathrm{Ru}\left(\mathrm{PEt}_{3}\right)_{3}\left(\mathrm{NTf}_{2}\right)_{2}(10 \mathrm{mg}, 0.010 \mathrm{mmol}, 5.0 \mathrm{~mol} \%)$ were combined with $\mathrm{DCB}(60 \mu \mathrm{L})$ were combined along with a magnetic stir bar in a one dram vial. The vial threads were wrapped with 1 layer of Teflon tape and the resulting mixture was heated at $80{ }^{\circ} \mathrm{C}$ with stirring for 48 hours. The reaction was allowed to cool, and all of the volatile materials were evaporated. The crude product was purified by silica gel chromatography, eluting with $40 \%$ ethyl acetate in hexanes. Yield $=30 \mathrm{mg}(52 \%) .{ }^{1} \mathrm{H}$ NMR $(600 \mathrm{MHz}$, chloroform- $d) \delta 7.91(\mathrm{~d}, J=2.3 \mathrm{~Hz}, 1 \mathrm{H})$, $7.23(\mathrm{dd}, J=8.5,2.4 \mathrm{~Hz}, 1 \mathrm{H}), 6.79(\mathrm{~d}, J=8.1 \mathrm{~Hz}, 1 \mathrm{H}), 6.72(\mathrm{dd}, J=8.1,2.0 \mathrm{~Hz}, 1 \mathrm{H}), 6.70(\mathrm{~d}, J$ $=2.0 \mathrm{~Hz}, 1 \mathrm{H}), 6.29(\mathrm{~d}, J=8.4 \mathrm{~Hz}, 1 \mathrm{H}), 4.23(\mathrm{~d}, J=8.6 \mathrm{~Hz}, 1 \mathrm{H}), 4.01$ (hept, $J=6.5 \mathrm{~Hz}, 1 \mathrm{H})$, $3.85(\mathrm{~d}, J=10.2 \mathrm{~Hz}, 6 \mathrm{H}), 2.83(\mathrm{dd}, J=13.5,5.3 \mathrm{~Hz}, 1 \mathrm{H}), 2.72(\mathrm{dd}, J=13.6,6.8 \mathrm{~Hz}, 1 \mathrm{H}), 2.17$ (s, $3 \mathrm{H}), 1.16(\mathrm{~d}, J=6.5 \mathrm{~Hz}, 3 \mathrm{H}) .{ }^{13} \mathrm{C} \mathrm{NMR}(151 \mathrm{MHz}$, chloroform- $d$ ) $\delta 156.5,148.9,148.0,147.7$, 138.5, 131.2, 121.7, 121.6, 113.0, 111.3, 107.1, 56.1, 56.0, 48.4, 42.3, 20.5, 17.5. GC-MS (EI+): 286 (M), 135 ([N-ethyl-5-methylpyridin-2-amine $\left.]^{\circ}\right)$.<smiles>Cc1ccc(NC(C)Cc2ccc(F)cc2)nc1</smiles>

\section{5-methyl- $N$-(1-(4-fluoro-phenyl)propan-2-yl)pyridine-2-amine (6a)}

p-Fluoro-allylbenzene (27 mg, $0.20 \mathrm{mmol}), 2$-amino-5-methylpyridine (22 $\mathrm{mg}, 0.20 \mathrm{mmol}$ ), and $\mathrm{Ru}\left(\mathrm{PEt}_{3}\right)_{3}\left(\mathrm{NTf}_{2}\right)_{2}(10 \mathrm{mg}, 0.010 \mathrm{mmol}, 5.0 \mathrm{~mol} \%)$ were combined with DCB $(60 \mu \mathrm{L})$ and a magnetic stir bar in a one dram vial. The vial threads were wrapped with 1 layer of Teflon tape and the resulting mixture was heated at $80{ }^{\circ} \mathrm{C}$ with stirring for 48 hours. The reaction was allowed to cool, and all of the volatile materials were evaporated. The crude product was purified by silica gel chromatography, eluting with $15 \%$ ethyl acetate in hexanes. Yield $=28 \mathrm{mg}(56 \%)$. ${ }^{1} \mathrm{H}$ NMR (600 MHz, chloroform- $d$ ) $\delta 7.92(\mathrm{~s}, 1 \mathrm{H}), 7.24(\mathrm{~d}, \mathrm{~J}=8.4 \mathrm{~Hz}, 1 \mathrm{H}), 7.14(\mathrm{dd}, \mathrm{J}=8.0,5.8$ $\mathrm{Hz}, 2 \mathrm{H}), 6.96(\mathrm{t}, \mathrm{J}=8.6 \mathrm{~Hz}, 2 \mathrm{H}), 6.29(\mathrm{~d}, \mathrm{~J}=8.4 \mathrm{~Hz}, 1 \mathrm{H}), 4.14(\mathrm{~d}, \mathrm{~J}=7.8 \mathrm{~Hz}, 1 \mathrm{H}), 4.01(\mathrm{~m}, 1 \mathrm{H})$, 2.87 (dd, J = 13.7, 5.2 Hz, 1H), 2.74 (dd, J = 13.6, 6.9 Hz, 1H), 2.17 (s, 3H), 1.14 (d, J = 6.4 Hz, $3 \mathrm{H}) .13 \mathrm{C}$ NMR $(101 \mathrm{MHz}$, chloroform- $d) \delta 162.9,160.5,156.3,148.0,138.5,134.3(\mathrm{~d}, J=3.2$ $\mathrm{Hz}), 131.1$ (d, $J=7.7 \mathrm{~Hz}), 121.7,115.2(\mathrm{~d}, J=21.1 \mathrm{~Hz}), 107.0,48.3$, 41.8, 20.3, 17.5. GC-MS (EI+): $244(\mathrm{M}), 229\left(\mathrm{M}-\mathrm{CH}_{3}\right), 135$ ([N-ethyl-5-methylpyridin-2-amine] $]^{\circ}$.<smiles>CCN(CC)C(=O)CCCCCCCC(C)Nc1ccc(C)cn1</smiles>

$N, N$-diethyl-9-((5-methylpyridin-2-yl)amino)decanamide (7a) 
9-decenoic acid diethyl amide (45 mg, $0.20 \mathrm{mmol}$ ), 2-amino-5-methylpyridine (22 $\mathrm{mg}, 0.20$ $\mathrm{mmol})$, and $\mathrm{Ru}\left(\mathrm{PEt}_{3}\right)_{3}\left(\mathrm{NTf}_{2}\right)_{2}(10 \mathrm{mg}, 0.010 \mathrm{mmol}, 5.0 \mathrm{~mol} \%)$ were combined with $\mathrm{DCB}(60 \mu \mathrm{L})$ and a magnetic stir bar in a one dram vial. The vial threads were wrapped with 1 layer of Teflon tape and the resulting mixture was heated at $80{ }^{\circ} \mathrm{C}$ with stirring for 48 hours. The reaction was allowed to cool, and all of the volatile materials were evaporated. The crude product was purified by silica gel chromatography, eluting with $40 \%$ ethyl acetate in hexanes. Yield $=44 \mathrm{mg}(66 \%)$. ${ }^{1} \mathrm{H}$ NMR $(600 \mathrm{MHz}$, chloroform-d) $\delta 7.86(\mathrm{~s}, 1 \mathrm{H}), 7.21$ (dd, J = 8.4, 2.4 Hz, 1H), 6.27 (d, J = 8.4 $\mathrm{Hz}, 1 \mathrm{H}), 4.18(\mathrm{~d}, \mathrm{~J}=8.6 \mathrm{~Hz}, 1 \mathrm{H}), 3.65(\mathrm{dq}, \mathrm{J}=8.3,6.2 \mathrm{~Hz}, 1 \mathrm{H}), 3.34(\mathrm{q}, \mathrm{J}=7.1 \mathrm{~Hz}, 2 \mathrm{H}), 3.27$ (q, $\mathrm{J}=7.1 \mathrm{~Hz}, 2 \mathrm{H}), 2.26(\mathrm{~d}, \mathrm{~J}=8.5 \mathrm{~Hz}, 1 \mathrm{H}), 2.24(\mathrm{~d}, \mathrm{~J}=8.5 \mathrm{~Hz}, 1 \mathrm{H}), 2.14(\mathrm{~s}, 3 \mathrm{H}), 1.60(\mathrm{p}, \mathrm{J}=7.5$ $\mathrm{Hz}, 2 \mathrm{H}), 1.50(\mathrm{~m}, 1 \mathrm{H}), 1.43(\mathrm{~m}, 1 \mathrm{H}), 1.39-1.21(\mathrm{~m}, 8 \mathrm{H}), 1.14(\mathrm{t}, \mathrm{J}=6.5 \mathrm{~Hz}, 6 \mathrm{H}, 2$ methyl groups), $1.08(\mathrm{t}, \mathrm{J}=7.1 \mathrm{~Hz}, 3 \mathrm{H}) .{ }^{13} \mathrm{C} \mathrm{NMR}(151 \mathrm{MHz}$, chloroform- $d$ ) $\delta 172.4,156.8,147.9$, 138.5, 121.1, 106.4, 47.5, 42.1, 40.1, 37.3, 33.2, 29.6, 29.5, 29.5, 26.1, 25.6, 21.1, 17.6, 14.5, 13.2. GC-MS (EI+): $333(\mathrm{M}), 318$ (M - Me), 135 ([N-ethyl-5-methylpyridin-2-amine $\left.]^{\circ}\right)$.

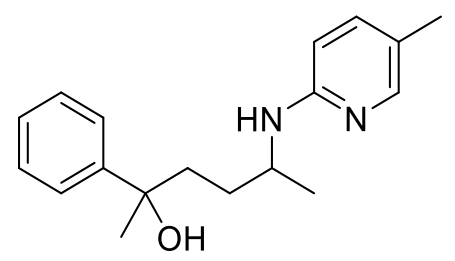

\section{5-((5-methylpyridin-2-yl)amino)-2-phenylhexan-2-ol (8a)}

2-phenylhex-5-en-2-ol (35 mg, $0.20 \mathrm{mmol})$, 2-amino-5-methylpyridine (22 $\mathrm{mg}, 0.20 \mathrm{mmol})$, and $\mathrm{Ru}\left(\mathrm{PEt}_{3}\right)_{3}\left(\mathrm{NTf}_{2}\right)_{2}(20 \mathrm{mg}, 0.020 \mathrm{mmol}, 10 \mathrm{~mol} \%)$ were combined with DCB $(60 \mu \mathrm{L})$ and a magnetic stir bar in a one dram vial. The vial threads were wrapped with 1 layer of Teflon tape and the resulting mixture was heated at $80{ }^{\circ} \mathrm{C}$ with stirring for 48 hours. The reaction was allowed to cool, and all of the volatile materials were evaporated. The crude product was purified by silica gel chromatography, eluting with $40 \%$ ethyl acetate in hexanes. Yield $=24 \mathrm{mg}(42 \%$, 52:48 dr). The peaks derived from diastereomer 1 are labelled and those derived from diastereomer 2 are given without labels. ${ }^{1} \mathrm{H}$ NMR $(400 \mathrm{MHz}$, chloroform- $d) \delta 7.88(\mathrm{~s}, 1 \mathrm{H}), 7.87$ (s, $1 \mathrm{H}$, diastereomer 1), $7.47(\mathrm{~d}, J=8.1 \mathrm{~Hz}, 4 \mathrm{H}$, both diastereomers), $7.34(\mathrm{~d}, J=7.7 \mathrm{~Hz}, 2 \mathrm{H}$, diastereomer 1), $7.32(\mathrm{~d}, \mathrm{~J}=7.7 \mathrm{~Hz}, 2 \mathrm{H}), 7.22(\mathrm{~m}, 4 \mathrm{H}$, both diastereomers), $6.26(\mathrm{~d}, J=8.5 \mathrm{~Hz}$, $2 \mathrm{H}$, both diastereomers), $4.11(\mathrm{~s}, 2 \mathrm{H}$, both diastereomers), 3.89 (h, $J=6.0 \mathrm{~Hz}, 2 \mathrm{H}$, both diastereomers), $2.16(\mathrm{~s}, 6 \mathrm{H}$, both diastereomers), $1.91(\mathrm{t}, \mathrm{J}=7.5 \mathrm{~Hz}, 4 \mathrm{H}$, both diastereomers) $1.52(\mathrm{~s}, 6 \mathrm{H}$, both diastereomers), $1.50-1.38(\mathrm{~m}, 4 \mathrm{H}$, both diastereomers), 1.25 (s, 2H, both diastereomers), 1.10 (d, $J=6.4 \mathrm{~Hz}, 6 \mathrm{H}$, both diastereomers). ${ }^{13} \mathrm{C}$ NMR (101 MHz, chloroform- $d$ ) $\delta 156.3$ (diastereomer 1), 156.1, 148.8, 148.4 (diastereomer 1), 146.6 (diastereomer 1), 146.6, 139.0 (diastereomer 1), 138.9, 128.2 (diastereomer 1), 128.2, 126.4 (diastereomer 1), 126.4, 125.0, 125.0 (diastereomer 1), 121.35, 121.30 (diastereomer 1), 107.90, 107.71 (diastereomer 1), 74.87, 74.62 (diastereomer 1), 48.1, 47.5 (diastereomer 1), 39.8 (diastereomer 1), 39.7, 32.6 (diastereomer 1), 32.1, 31.4 (diastereomer 1), 31.1, 29.9, 21.7, 21.4 (diastereomer 1), 17.5 (diastereomer 1). GC-MS (EI+): 284 (M), 135 ([N-ethyl-5-methylpyridin-2-amine]").<smiles>Cc1ccc(NC(C)C2CC=CCC2)nc1</smiles> 


\section{$N$-(1-(cyclohex-3-en-1-yl)ethyl)-5-methylpyridin-2-amine (9a)}

4-ethenyl-cyclohexene (26 $\mu \mathrm{L}, 22 \mathrm{mg}, 0.20 \mathrm{mmol}$ ), 2-amino-5-methylpyridine (22 $\mathrm{mg}, 0.20$ $\mathrm{mmol})$, and $\mathrm{Ru}\left(\mathrm{PEt}_{3}\right)_{3}\left(\mathrm{NTf}_{2}\right)_{2}(20 \mathrm{mg}, 0.020 \mathrm{mmol}, 10 \mathrm{~mol} \%)$ were combined with $\mathrm{DCB}(60 \mu \mathrm{L})$ and a magnetic stir bar in a one dram vial. The vial threads were wrapped with 1 layer of Teflon tape and the resulting mixture was heated at $100{ }^{\circ} \mathrm{C}$ with stirring for 48 hours. The reaction was allowed to cool, and all of the volatile materials were evaporated. The crude product was purified by silica gel chromatography, eluting with $15 \%$ ethyl acetate in hexanes. Yield $=27 \mathrm{mg}(61 \%)$. ${ }^{1} \mathrm{H}$ NMR (600 MHz, chloroform- $d$ ) $\delta$ Major isomer: 7.88 (s, 1H, major), 7.22 (dd, $J=8.5,2.4 \mathrm{~Hz}$, $1 \mathrm{H}$, major), 6.30 (d, $J=8.4 \mathrm{~Hz}, 1 \mathrm{H}$, major), 5.67 (m, 2H, major), 4.19 (d, $J=9.3 \mathrm{~Hz}, 1 \mathrm{H}$, major), 3.67 (h, J = $7 \mathrm{~Hz}, 1 \mathrm{H}$, major), $2.20-1.98$ (m, 3H, major), 2.16 (s, 3H, major), $1.88-1.83$ (m, $2 \mathrm{H}$, major), $1.69(\mathrm{~m}, 1 \mathrm{H}$, major), $1.28(\mathrm{~m}, 1 \mathrm{H}$, major), $1.16(\mathrm{t}, J=6.4 \mathrm{~Hz}, 3 \mathrm{H}$, major). Minor isomer: $7.88(\mathrm{~s}, 1 \mathrm{H}), 7.22(\mathrm{dd}, J=8.5,2.4 \mathrm{~Hz}, 1 \mathrm{H}), 6.29(\mathrm{~d}, J=8.4 \mathrm{~Hz}, 1 \mathrm{H}), 5.67(\mathrm{~m}, 2 \mathrm{H}), 4.24$ $(\mathrm{d}, J=9.3 \mathrm{~Hz}, 1 \mathrm{H}), 3.67(\mathrm{~h}, \mathrm{~J}=7 \mathrm{~Hz}, 1 \mathrm{H}), 2.20-1.98(\mathrm{~m}, 3 \mathrm{H}), 2.16(\mathrm{~s}, 3 \mathrm{H}), 1.90(\mathrm{~m}, 1 \mathrm{H}), 1.78$ $(\mathrm{m}, 1 \mathrm{H}), 1.71(\mathrm{~m}, 1 \mathrm{H}), 1.36(\mathrm{~m}, 1 \mathrm{H}), 1.16(\mathrm{t}, J=6.4 \mathrm{~Hz}, 3 \mathrm{H}) .{ }^{13} \mathrm{C}$ NMR $(151 \mathrm{MHz}$, chloroform- $d)$ $\delta$ 157.0, 156.9 (major), 147.8 (major), 147.8, 138.5 (major), 138.5, 127.2, 127.1 (major), 126.4 (major), 126.4, 121.1, 121.1 (major), 106.5, 106.4 (major), 51.3, 51.1 (major), 39.7, 39.5 (major), 28.3 (major), 28.0, 25.8, 25.5 (major), 25.4, 25.0 (major), 18.1, 18.0 (major), 17.4, 17.4(major). GC-MS (EI+): 216 (M), 201 (M - Me), 135 ([N-ethyl-5-methylpyridin-2-amine $\left.]^{\circ}\right)$.

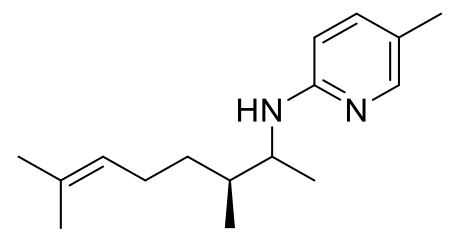

\section{$N$-((3S)-3,7-dimethyloct-6-en-2-yl)-5-methylpyridin-2-amine (10a)}

(+)- $\beta$-Citronellene (28 mg, $0.20 \mathrm{mmol}), 2$-amino-5-methylpyridine $(22 \mathrm{mg}, 0.20 \mathrm{mmol}$ ), and $\mathrm{Ru}\left(\mathrm{PEt}_{3}\right)_{3}\left(\mathrm{NTf}_{2}\right)_{2}(20 \mathrm{mg}, 0.020 \mathrm{mmol}, 10 \mathrm{~mol} \%)$ were combined with DCB $(60 \mu \mathrm{L})$ and a magnetic stir bar in a one dram vial. The vial threads were wrapped with 1 layer of Teflon tape and the resulting mixture was heated at $100{ }^{\circ} \mathrm{C}$ with stirring for 48 hours. The reaction was allowed to cool, and all of the volatile materials were evaporated. The crude product was purified by silica gel chromatography, eluting with $20 \%$ ethyl acetate in hexanes. Yield $=27 \mathrm{mg}(54 \%$, 7:4 dr). ${ }^{1} \mathrm{H}$ NMR $(600 \mathrm{MHz}$, chloroform- $d){ }^{1} \mathrm{H}$ NMR $(600 \mathrm{MHz}$, Chloroform-d) $\delta 7.87(\mathrm{~s}, 1 \mathrm{H}$, major), 7.87 (s, 1H, minor), 7.22 (d, $J=8.2 \mathrm{~Hz}, 1 \mathrm{H}$, major), 7.22 (d, $J=8.2 \mathrm{~Hz}, 1 \mathrm{H}$, minor), 6.28 (d, $J=8.5 \mathrm{~Hz}, 1 \mathrm{H}$, major), 6.27 (d, J = 8.4 Hz, 1H, minor), 5.10 (ddt, $J=7.1,5.7,1.5 \mathrm{~Hz}, 1 \mathrm{H}$, minor), 5.05 (tt, $J=7.2,1.5 \mathrm{~Hz}, 1 \mathrm{H}$, major), 4.31 (d, $J=9.1 \mathrm{~Hz}, 1 \mathrm{H}$, minor), 4.27 (d, $J=9.1 \mathrm{~Hz}$, $1 \mathrm{H}$, major), 3.68 (dtd, $J=13.1,6.6,3.2 \mathrm{~Hz}, 1 \mathrm{H}$, major), 3.63 (ddd, $J=8.9,6.8,5.0 \mathrm{~Hz}, 1 \mathrm{H}$, minor), 2.15 (s, 3H, major), 2.15 (s, 3H, minor), 2.06 (m, 1H, minor), 2.04 (m, 1H, major), 1.96 (m, 1H, minor), $1.92(\mathrm{~m}, 1 \mathrm{H}$, major), $1.68(\mathrm{~s}, 3 \mathrm{H}$, minor), $1.67(\mathrm{~m}, 1 \mathrm{H}$, minor), $1.65(\mathrm{~m}, 1 \mathrm{H}$, major), 1.65 (s, 3H, major), 1.60 (s, 3H, minor), 1.57 (s, 3H, major), 1.48 (m, 1H, minor), 1.46 (m, 1H, major), $1.19(\mathrm{~m}, 1 \mathrm{H}$, minor), $1.18(\mathrm{~m}, 1 \mathrm{H}$, major), $1.12(\mathrm{~d}, J=6.6 \mathrm{~Hz}, 3 \mathrm{H}$, major), 1.09 (d, $J=6.6 \mathrm{~Hz}, 3 \mathrm{H}$, minor), $0.95\left(\mathrm{~d}, J=6.9 \mathrm{~Hz}, 3 \mathrm{H}\right.$, major), $0.89\left(\mathrm{~d}, J=6.8 \mathrm{~Hz}, 3 \mathrm{H}\right.$, minor). ${ }^{13} \mathrm{C}$ NMR (151 MHz, chloroform-d) $\delta 157.0$ (major), 156.8 (minor), 148.0 (minor), 147.9 (major), 138.5 (minor), 138.5 (major), 131.6 (minor), 131.5 (major), 124.8 (major), 124.7 (minor), 121.1 (minor), 121.1 (major), 106.3 (major), 106.3 (minor), 51.2 (minor), 51.1 (major), 37.5 (major), 36.9 (minor), 33.8 (minor), 32.8 (major), 26.0 (minor), 26.0 (major), 25.8 (minor), 25.8 (major), 
17.8 (major), 17.8 (minor), 17.5 (major), 16.4 (minor), 15.5 (major), 14.5 (minor). GC-MS (EI+): 246 (M), 135 ([N-ethyl-5-methylpyridin-2-amine $]^{\circ}$ ).<smiles>C/C=C/CCC(C)Nc1ccc(C)cn1</smiles>

(E)-N-(hept-5-en-2-yl)-5-methylpyridin-2-amine (11a)

1,5-heptadiene (19 mg, $0.20 \mathrm{mmol}), 2$-amino-5-methylpyridine (22 $\mathrm{mg}, 0.20 \mathrm{mmol})$, and $\mathrm{Ru}\left(\mathrm{PEt}_{3}\right)_{3}\left(\mathrm{NTf}_{2}\right)_{2}(10 \mathrm{mg}, 0.010 \mathrm{mmol}, 5.0 \mathrm{~mol} \%)$ were combined with $\mathrm{DCB}(60 \mu \mathrm{L})$ and a magnetic stir bar in a one dram vial. The vial threads were wrapped with 1 layer of Teflon tape and the resulting mixture was heated at $80{ }^{\circ} \mathrm{C}$ with stirring for 48 hours. The reaction was allowed to cool, and all of the volatile materials were evaporated. The crude product was purified by silica gel chromatography, eluting with $15 \%$ ethyl acetate in hexanes. Yield $=27 \mathrm{mg}(67 \%)$. ${ }^{1} \mathrm{H}$ NMR (600 MHz, chloroform- $\left.d\right) \delta 7.88(\mathrm{~s}, 1 \mathrm{H}), 7.23(\mathrm{~d}, \mathrm{~J}=8.5 \mathrm{~Hz}, 1 \mathrm{H}), 6.28(\mathrm{~d}, \mathrm{~J}=8.3 \mathrm{~Hz}$, $1 \mathrm{H}), 5.50-5.30(\mathrm{~m}, 2 \mathrm{H}), 4.19(\mathrm{bs}, 1 \mathrm{H}), 3.69$ (dt, J = 13.8, 7.0 Hz, 1H), $2.16(\mathrm{~s}, 3 \mathrm{H}), 2.14-2.00$ $(\mathrm{m}, 2 \mathrm{H}), 1.71-1.43(\mathrm{~m}, 5 \mathrm{H}), 1.18(\mathrm{~d}, \mathrm{~J}=6.4 \mathrm{~Hz}, 3 \mathrm{H}) .{ }^{13} \mathrm{C}$ NMR $(151 \mathrm{MHz}$, chloroform- $d) \delta$ 156.6, 147.7, 138.3, 130.6, 125.2, 121.0, 106.2, 46.9, 37.0, 29.1, 20.8, 17.8, 17.3. GC-MS (EI+): 204 (M), 149 (M-butene), 135 ([N-ethyl-5-methylpyridin-2-amine $\left.]^{\circ}\right)$.<smiles>Cc1ccc(NC(C)CCCCCCCC(=O)O[C@H]2CC[C@H]3[C@H]4CCC5=CC(=O)C=C[C@]5(C)[C@H]4CC[C@]23C)nc1</smiles>

\section{Aminopyridyl boldenone undecylenate (12a)}

Boldenone undecylenate $(91 \mathrm{mg}, 0.20 \mathrm{mmol}), 2$-amino-5-methylpyridine (22 $\mathrm{mg}, 0.20 \mathrm{mmol})$, and $\mathrm{Ru}\left(\mathrm{PEt}_{3}\right)_{3}\left(\mathrm{NTf}_{2}\right)_{2}(30 \mathrm{mg}, 0.030 \mathrm{mmol}, 15 \mathrm{~mol} \%)$ were combined with DCB $(60 \mu \mathrm{L})$ and a magnetic stir bar in a one dram vial. The vial threads were wrapped with 1 layer of Teflon tape and the resulting mixture was heated at $80{ }^{\circ} \mathrm{C}$ with stirring for 48 hours. The reaction was allowed to cool, and all of the volatile materials were evaporated. The crude product was purified by silica gel chromatography, eluting with a gradient of $20 \%$ to $60 \%$ ethyl acetate in hexanes. Yield $=38 \mathrm{mg}(34 \%)$. Both diastereomers gave identical NMR and mass spectra. ${ }^{1} \mathrm{H}$ NMR $(500$ MHz, chloroform- $d$ ) $\delta 7.87(\mathrm{~s}, 1 \mathrm{H}), 7.23(\mathrm{dd}, 1 \mathrm{H}), 7.03(\mathrm{~d}, J=10.2 \mathrm{~Hz}, 1 \mathrm{H}), 6.28(\mathrm{~d}, J=8.5 \mathrm{~Hz}$, $1 \mathrm{H}), 6.22(\mathrm{dd}, J=10.1,1.5 \mathrm{~Hz}, 1 \mathrm{H}), 6.06(\mathrm{~s}, 1 \mathrm{H}), 4.58(\mathrm{t}, J=8.5 \mathrm{~Hz}, 1 \mathrm{H}), 4.18(\mathrm{~d}, J=8.4 \mathrm{~Hz}$, $1 \mathrm{H}), 3.66$ (hept, $J=6.6 \mathrm{~Hz}, 1 \mathrm{H}), 2.46(\mathrm{td}, J=13.4,4.7 \mathrm{~Hz}, 1 \mathrm{H}), 2.36(\mathrm{~d}, J=12.6 \mathrm{~Hz}, 1 \mathrm{H}), 2.27$ $(\mathrm{t}, J=7.5 \mathrm{~Hz}, 2 \mathrm{H}), 2.15(\mathrm{~s}, 3 \mathrm{H}), 1.94(\mathrm{~d}, J=14.2 \mathrm{~Hz}, 2 \mathrm{H}), 1.86-1.54(\mathrm{~m}, 9 \mathrm{H}), 1.54-1.41(\mathrm{~m}$, $4 \mathrm{H}), 1.38-1.32(\mathrm{~m}, 2 \mathrm{H}), 1.31-1.24(\mathrm{~m}, 6 \mathrm{H}), 1.22(\mathrm{~s}, 3 \mathrm{H}), 1.16(\mathrm{~d}, J=6.3 \mathrm{~Hz}, 3 \mathrm{H}), 1.10-0.98$ $(\mathrm{m}, 4 \mathrm{H}), 0.85(\mathrm{~s}, 3 \mathrm{H}) .{ }^{13} \mathrm{C}$ NMR $(151 \mathrm{MHz}$, chloroform- $d) \delta 186.4,173.9,168.9,156.2,155.8$, 146.5, 139.3, 127.6, 124.0, 121.2, 106.8, 82.1, 52.3, 50.0, 47.6, 43.6, 42.8, 37.2, 36.6, 35.4, 34.6, 33.1, 32.8, 29.6, 29.4, 29.2, 29.2, 27.5, 26.1, 25.1, 23.8, 22.4, 20.9, 18.8, 17.4, 12.2. ESI-MS (+): Calc. for $\mathrm{C}_{36} \mathrm{H}_{53} \mathrm{O}_{3} \mathrm{~N}_{2}$ : 561.4051. Found: 563.4048 . 
<smiles>Cc1ccnc(NC(C)C)c1</smiles>

\section{4-methyl- $N$-(dodecan-2-yl)pyridine-2-amine (13a)}

1-Dodecene (44 $\mu \mathrm{L}, 34 \mathrm{mg}, 0.20 \mathrm{mmol}$ ), 2-amino-4-methylpyridine (22 $\mathrm{mg}, 0.20 \mathrm{mmol}$ ), and $\mathrm{Ru}\left(\mathrm{PEt}_{3}\right)_{3}\left(\mathrm{NTf}_{2}\right)_{2}(10 \mathrm{mg}, 0.010 \mathrm{mmol}, 5.0 \mathrm{~mol} \%)$ were combined with $\mathrm{DCB}(60 \mu \mathrm{L})$ and a magnetic stir bar in a one dram vial. The vial threads were wrapped with 1 layer of Teflon tape and the resulting mixture was heated at $80{ }^{\circ} \mathrm{C}$ with stirring for 48 hours. The reaction was allowed to cool, and all of the volatile materials were evaporated. The crude product was purified by silica gel chromatography, eluting with $15 \%$ ethyl acetate in hexanes. Yield $=35 \mathrm{mg}(63 \%)$. ${ }^{1} \mathrm{H}$ NMR $(500 \mathrm{MHz}$, Chloroform- $d$ ) $\delta 7.94(\mathrm{~d}, J=5.2 \mathrm{~Hz}, 1 \mathrm{H}), 6.39(\mathrm{~d}, J=5.2 \mathrm{~Hz}, 1 \mathrm{H}), 6.18(\mathrm{~s}$, $1 \mathrm{H}), 4.29(\mathrm{~d}, J=8.5 \mathrm{~Hz}, 1 \mathrm{H}), 3.72(\mathrm{dq}, J=8.4,6.3 \mathrm{~Hz}, 1 \mathrm{H}), 2.24(\mathrm{~s}, 3 \mathrm{H}), 1.60-1.50(\mathrm{~m}, 1 \mathrm{H})$, $1.51-1.43(\mathrm{~m}, 1 \mathrm{H}), 1.43-1.35(\mathrm{~m}, 2 \mathrm{H}), 1.20-1.30(\mathrm{~m}, 14 \mathrm{H}), 1.20(\mathrm{~d}, J=6.3 \mathrm{~Hz}, 3 \mathrm{H}), 0.90(\mathrm{t}, J$ $=6.9 \mathrm{~Hz}, 3 \mathrm{H}) .{ }^{13} \mathrm{C}$ NMR $(126 \mathrm{MHz}$, Chloroform- $d$ ) $\delta 158.6,148.2,147.9,113.9,106.7,47.1$, 37.3, 31.9, 29.6, 29.6, 29.3, 26.0, 22.7, 21.2, 21.0, 14.1. GC-MS (EI+): 276 (M), $261\left(\mathrm{M}-\mathrm{CH}_{3}\right)$, 135 ([N-ethyl-5-methylpyridin-2-amine $]^{\circ}$ ).<smiles>CCCCCCC(C)Nc1ccc(OC)cn1</smiles>

\section{5-methoxy- $N$-(octan-2-yl)pyridin-2-amine (14a)}

1-octene $(31 \mu \mathrm{L}, 23 \mathrm{mg}, 0.20 \mathrm{mmol})$, 5-methoxypyridin-2-amine $(25 \mathrm{mg}, 0.20 \mathrm{mmol})$, and $\mathrm{Ru}\left(\mathrm{PEt}_{3}\right)_{3}\left(\mathrm{NTf}_{2}\right)_{2}(10 \mathrm{mg}, 0.010 \mathrm{mmol}, 5.0 \mathrm{~mol} \%)$ were combined with DCB $(60 \mu \mathrm{L})$ and a magnetic stir bar in a one dram vial. The vial threads were wrapped with 1 layer of Teflon tape and the resulting mixture was heated at $80{ }^{\circ} \mathrm{C}$ with stirring for 48 hours. The reaction was allowed to cool, and all of the volatile materials were evaporated. The crude product was purified by silica gel chromatography, eluting with $25 \%$ ethyl acetate in hexanes. Yield $=32 \mathrm{mg}(68 \%)$. ${ }^{1} \mathrm{H}$ NMR $(600 \mathrm{MHz}$, chloroform- $d) \delta 7.80(\mathrm{~d}, J=3.0 \mathrm{~Hz}, 1 \mathrm{H}), 7.09(\mathrm{dd}, J=8.9,3.0 \mathrm{~Hz}, 1 \mathrm{H})$, $6.33(\mathrm{~d}, J=9.0 \mathrm{~Hz}, 1 \mathrm{H}), 4.05(\mathrm{~s}, 1 \mathrm{H}), 3.77(\mathrm{~s}, 3 \mathrm{H}), 3.64(\mathrm{dt}, J=11.2,5.1 \mathrm{~Hz}, 2 \mathrm{H}), 1.53$ (dddd, $J$ $=12.8,9.9,6.3,5.1 \mathrm{~Hz}, 1 \mathrm{H}), 1.49-1.40(\mathrm{~m}, 1 \mathrm{H}), 1.41-1.31(\mathrm{~m}, 2 \mathrm{H}), 1.33-1.21(\mathrm{~m}, 6 \mathrm{H}), 1.17$ $(\mathrm{d}, J=6.4 \mathrm{~Hz}, 3 \mathrm{H}), 0.87(\mathrm{t}, J=7.0 \mathrm{~Hz}, 3 \mathrm{H}) .{ }^{13} \mathrm{C}$ NMR $(151 \mathrm{MHz}$, chloroform- $d) \delta 153.8,148.5$, 133.8, 125.8, 107.3, 56.7, 48.0, 37.4, 31.9, 29.5, 26.2, 22.7, 21.1, 14.2. GC-MS (EI+): 236 (M), $221\left(\mathrm{M}-\mathrm{CH}_{3}\right), 151$ ([N-ethyl-5-methoxypyridin-2-amine $\left.]^{\circ}\right)$.

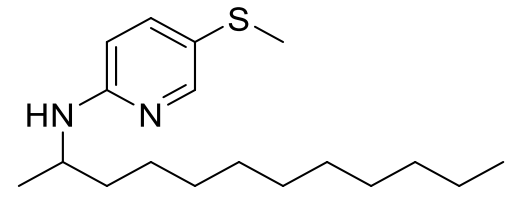

$\mathrm{N}$-(dodecan-2-yl)-5-(methylthio)pyridin-2-amine (15a)

1-dodecene (34 mg, $0.20 \mathrm{mmol}$ ), 5-(methylthio)pyridin-2-amine (28 mg, $0.20 \mathrm{mmol}$ ), and $\mathrm{Ru}\left(\mathrm{PEt}_{3}\right)_{3}\left(\mathrm{NTf}_{2}\right)_{2}(10 \mathrm{mg}, 0.010 \mathrm{mmol}, 5.0 \mathrm{~mol} \%)$ were combined with DCB $(60 \mu \mathrm{L})$ and a magnetic stir bar in a one dram vial. The vial threads were wrapped with 1 layer of Teflon tape 
and the resulting mixture was heated at $100{ }^{\circ} \mathrm{C}$ with stirring for 48 hours. The reaction was allowed to cool, and all of the volatile materials were evaporated. The crude product was purified by silica gel chromatography, eluting with $40 \%$ ethyl acetate in hexanes. Yield $=26 \mathrm{mg}(42 \%)$. ${ }^{1} \mathrm{H}$ NMR (600 MHz, chloroform- $d$ ) $\delta 8.12(\mathrm{~d}, J=2.2 \mathrm{~Hz}, 1 \mathrm{H}), 7.47(\mathrm{dd}, J=8.7,2.4 \mathrm{~Hz}, 1 \mathrm{H})$, $6.29(\mathrm{~d}, J=8.7 \mathrm{~Hz}, 1 \mathrm{H}), 4.44(\mathrm{~d}, J=8.3 \mathrm{~Hz}, 1 \mathrm{H}), 3.73-3.66(\mathrm{~m}, 1 \mathrm{H}), 2.35$ (s, 3H), 1.49 (dtdd, $J$ $=25.4,13.2,9.7,5.9 \mathrm{~Hz}, 2 \mathrm{H}), 1.34(\mathrm{dtt}, J=18.8,8.4,4.9 \mathrm{~Hz}, 2 \mathrm{H}), 1.29-1.22(\mathrm{~m}, 14 \mathrm{H}), 1.17(\mathrm{~d}$, $J=6.4 \mathrm{~Hz}, 3 \mathrm{H}), 0.87(\mathrm{t}, J=7.0 \mathrm{~Hz}, 3 \mathrm{H}) .{ }^{13} \mathrm{C} \mathrm{NMR}(151 \mathrm{MHz}$, chloroform- $d) \delta 157.6,151.4$, 141.3, 119.9, 106.9, 47.4, 37.3, 32.0, 29.7, 29.7, 29.7, 29.4, 26.1, 22.7, 21.0, 20.4, 14.2. GC-MS (EI+): 308 (M), $292\left(\mathrm{M}-\mathrm{CH}_{4}\right), 167$ ([N-ethyl-5-(methylthio)pyridin-2-amine] $\left.]^{\circ}\right)$.

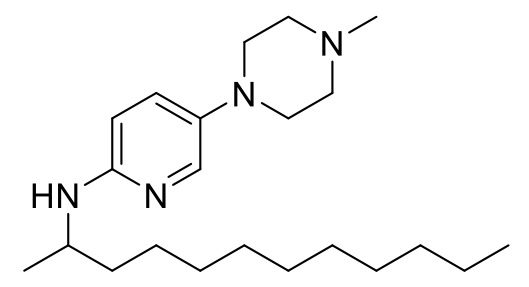

\section{$N$-(dodecan-2-yl)-5-(4-methylpiperazin-1-yl)pyridin-2-amine (16a)}

1-dodecene (34 mg, $0.20 \mathrm{mmol}$ ), 5-(4-methylpiperazin-1-yl)pyridin-2-amine (39 mg, 0.20 $\mathrm{mmol})$, and $\mathrm{Ru}\left(\mathrm{PEt}_{3}\right)_{3}\left(\mathrm{NTf}_{2}\right)_{2}(10 \mathrm{mg}, 0.010 \mathrm{mmol}, 5.0 \mathrm{~mol} \%)$ were combined with $\mathrm{DCB}(60 \mu \mathrm{L})$ and a magnetic stir bar in a one dram vial. The vial threads were wrapped with 1 layer of Teflon tape and the resulting mixture was heated at $100{ }^{\circ} \mathrm{C}$ with stirring for 48 hours. The reaction was allowed to cool, and all of the volatile materials were evaporated. The crude product was purified by silica gel chromatography, eluting with $5 \% \mathrm{MeOH}$ in DCM. Yield $=34 \mathrm{mg}(47 \%)$. ${ }^{1} \mathrm{H}$ NMR $(600 \mathrm{MHz}$, chloroform- $d) \delta 7.78(\mathrm{~d}, J=2.8 \mathrm{~Hz}, 1 \mathrm{H}), 7.18(\mathrm{dd}, J=9.0,2.9 \mathrm{~Hz}, 1 \mathrm{H}), 6.33(\mathrm{~d}, J=$ $8.9 \mathrm{~Hz}, 1 \mathrm{H}), 4.18(\mathrm{~s}, 1 \mathrm{H}), 3.63(\mathrm{~h}, J=6.4 \mathrm{~Hz}, 1 \mathrm{H}), 3.06-3.01(\mathrm{~m}, 4 \mathrm{H}), 2.60-2.55(\mathrm{~m}, 4 \mathrm{H})$, $2.35(\mathrm{~s}, 3 \mathrm{H}), 1.52(\mathrm{ddd}, J=16.1,12.2,5.7 \mathrm{~Hz}, 1 \mathrm{H}), 1.44(\mathrm{ddd}, J=13.0,6.4,4.2 \mathrm{~Hz}, 1 \mathrm{H}), 1.41-$ $1.31(\mathrm{~m}, 2 \mathrm{H}), 1.30-1.23(\mathrm{~m}, 14 \mathrm{H}), 1.16(\mathrm{~d}, J=6.3 \mathrm{~Hz}, 3 \mathrm{H}), 0.87(\mathrm{t}, J=7.0 \mathrm{~Hz}, 3 \mathrm{H}) .{ }^{13} \mathrm{C} \mathrm{NMR}$ (151 MHz, chloroform- $d$ ) $\delta 153.9,139.4,137.5,129.3,107.1,55.3,51.1,47.8,46.2,37.4,32.0$,

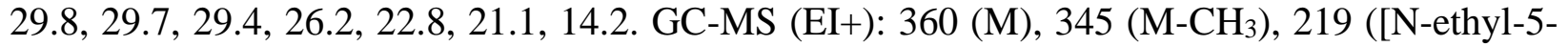
(4-methylpiperazin-1-yl)pyridin-2-amine] $]^{\circ}$.<smiles>Cc1ccnc(NC(C)C2CCCCC2)c1</smiles>

\section{$N$-(1-cyclohexylethyl)-4-methylpyridin-2-amine (17a)}

Vinylcyclohexane $(27 \mu \mathrm{L}, 22 \mathrm{mg}, 0.20 \mathrm{mmol}), 2$-amino-4-methylpyridine (22 $\mathrm{mg}, 0.20 \mathrm{mmol})$, $\mathrm{Ru}\left(\mathrm{PEt}_{3}\right)_{3}\left(\mathrm{NTf}_{2}\right)_{2}(20 \mathrm{mg}, 0.010 \mathrm{mmol}, 10 \mathrm{~mol} \%), 1,2-\mathrm{DCB}(60 \mu \mathrm{L})$ and a magnetic stir bar were combined in a one dram vial. The vial threads were wrapped with 1 layer of Teflon tape and the resulting mixture was heated at $100{ }^{\circ} \mathrm{C}$ with stirring for 48 hours. The mixture was allowed to cool, and all of the volatile materials were removed. The crude product was purified by silica gel chromatography, eluting with $20 \%$ ethyl acetate in hexanes. Yield $=28 \mathrm{mg}(63 \%)$. ${ }^{1} \mathrm{H}$ NMR (500 MHz, Chloroform- $\left.d\right) \delta 7.96(\mathrm{~d}, J=5.2 \mathrm{~Hz}, 1 \mathrm{H}), 6.40(\mathrm{dd}, J=5.2,1.4 \mathrm{~Hz}, 1 \mathrm{H})$, $6.20(\mathrm{~s}, 1 \mathrm{H}), 4.38(\mathrm{~d}, J=9.1 \mathrm{~Hz}, 1 \mathrm{H}), 3.64(\mathrm{dt}, J=9.1,6.3 \mathrm{~Hz}, 1 \mathrm{H}), 2.27(\mathrm{~s}, 3 \mathrm{H}), 1.95-1.65$ (m, 
$5 \mathrm{H}), 1.46(\mathrm{ddp}, J=14.6,8.6,3.0 \mathrm{~Hz}, 1 \mathrm{H}), 1.35-0.99(\mathrm{~m}, 9 \mathrm{H}) \cdot{ }^{13} \mathrm{C}$ NMR $(126 \mathrm{MHz}$, Chloroform- $d$ ) $\delta 159.2,148.6,148.2,114.2,107.1,51.9,43.8,29.9,29.2,26.9,26.8,26.7,21.67$, 18.2. GC-MS (EI+): $218(\mathrm{M}), 203\left(\mathrm{M}-\mathrm{CH}_{3}\right)$.<smiles>Cc1ccnc(NC(C)c2ccccc2)c1</smiles>

\section{4-methyl- $N$-(1-phenylethyl)pyridin-2-amine (18a)}

Styrene (23 $\mu \mathrm{L}, 21 \mathrm{mg}, 0.20 \mathrm{mmol}), 2$-amino-4-methylpyridine (22 $\mathrm{mg}, 0.20 \mathrm{mmol}$ ), $\mathrm{Ru}\left(\mathrm{PEt}_{3}\right)_{3}\left(\mathrm{NTf}_{2}\right)_{2}(20 \mathrm{mg}, 0.010 \mathrm{mmol}, 10 \mathrm{~mol} \%), 1,2-\mathrm{DCB}(60 \mu \mathrm{L})$ and a magnetic stir bar were combined in a one dram vial. The vial threads were wrapped with 1 layer of Teflon tape and the resulting mixture was heated at $100{ }^{\circ} \mathrm{C}$ with stirring for 48 hours. The mixture was allowed to cool, and all of the volatile materials were removed. The crude product was purified by silica gel chromatography, eluting with $30 \%$ ethyl acetate in hexanes. Yield $=27 \mathrm{mg}(62 \%)$. ${ }^{1} \mathrm{H}$ NMR $(600 \mathrm{MHz}$, Chloroform- $d$ ) $\delta 7.93(\mathrm{dd}, J=5.2,0.8 \mathrm{~Hz}, 1 \mathrm{H}), 7.40-7.36(\mathrm{~m}, 2 \mathrm{H}), 7.32$ $(\mathrm{m}, J=8.5,6.9 \mathrm{~Hz}, 2 \mathrm{H}), 7.25-7.21(\mathrm{~m}, 1 \mathrm{H}), 6.39(\mathrm{ddd}, J=5.1,1.4,0.7 \mathrm{~Hz}, 1 \mathrm{H}), 6.03(\mathrm{dt}, J=$ $1.6,0.8 \mathrm{~Hz}, 1 \mathrm{H}), 4.92(\mathrm{~d}, J=6.4 \mathrm{~Hz}, 1 \mathrm{H}), 4.74(\mathrm{p}, J=6.71 \mathrm{~Hz}, 1 \mathrm{H}), 2.13(\mathrm{~d}, J=0.7 \mathrm{~Hz}, 3 \mathrm{H})$, $1.54(\mathrm{~d}, J=6.8 \mathrm{~Hz}, 3 \mathrm{H}) .{ }^{13} \mathrm{C}$ NMR $\left(151 \mathrm{MHz}, \mathrm{CDCl}_{3}\right) \delta 158.3,148.5,147.9,144.9,128.75$, 127.0, 125.9, 114.7, 107.1, 51.9, 24.4, 21.3. GC-MS (EI+): $212(\mathrm{M}), 197\left(\mathrm{M}_{-} \mathrm{CH}_{3}\right)$.<smiles>Cc1ccc(C(C)Nc2cc(C)ccn2)cc1</smiles>

\section{4-methyl-N-(1-(p-tolyl)ethyl)pyridin-2-amine (19a)}

4-Methylstyrene ( $26 \mu \mathrm{L}, 24 \mathrm{mg}, 0.20 \mathrm{mmol}), 2$-amino-4-methylpyridine ( $22 \mathrm{mg}, 0.20 \mathrm{mmol}$ ), $\mathrm{Ru}\left(\mathrm{PEt}_{3}\right)_{3}\left(\mathrm{NTf}_{2}\right)_{2}(20 \mathrm{mg}, 0.010 \mathrm{mmol}, 10 \mathrm{~mol} \%), 1,2-\mathrm{DCB}(60 \mu \mathrm{L})$ and a magnetic stir bar were combined in a one dram vial. The vial threads were wrapped with 1 layer of Teflon tape and the resulting mixture was heated at $100{ }^{\circ} \mathrm{C}$ with stirring for 48 hours. The mixture was allowed to cool, and all of the volatile materials were removed. The crude product was purified by silica gel chromatography, eluting with $30 \%$ ethyl acetate in hexanes. Yield $=27 \mathrm{mg}(59 \%)$. ${ }^{1} \mathrm{H}$ NMR $(600 \mathrm{MHz}$, Chloroform- $d$ ) $\delta 7.94(\mathrm{~d}, J=5.2 \mathrm{~Hz}, 1 \mathrm{H}), 7.28(\mathrm{~d}, J=8.0 \mathrm{~Hz}, 2 \mathrm{H}), 7.14$ (d, $J=7.8 \mathrm{~Hz}, 2 \mathrm{H}), 6.39(\mathrm{dd}, J=5.2,1.4 \mathrm{~Hz}, 1 \mathrm{H}), 6.05(\mathrm{~s}, 1 \mathrm{H}), 5.07(\mathrm{~d}, J=6.5 \mathrm{~Hz}, 1 \mathrm{H}), 4.72(\mathrm{p}, J=$ $6.6 \mathrm{~Hz}, 1 \mathrm{H}), 2.33(\mathrm{~s}, 3 \mathrm{H}), 2.14(\mathrm{~s}, 3 \mathrm{H}), 1.53(\mathrm{~d}, J=6.8 \mathrm{~Hz}, 3 \mathrm{H}) .{ }^{13} \mathrm{C} \mathrm{NMR}\left(126 \mathrm{MHz}, \mathrm{CDCl}_{3}\right) \delta$ $158.3,148.7,147.7,141.9,136.6,129.4,125.9,114.7,107.1,51.6,24.5,21.4,21.1$. GC-MS (EI+): $226(\mathrm{M}), 211\left(\mathrm{M}-\mathrm{CH}_{3}\right)$. 
<smiles>Cc1ccnc(NC(C)c2ccc(F)cc2)c1</smiles>

\section{N-(1-(4-fluorophenyl)ethyl)-4-methylpyridin-2-amine (20a)}

4-Fluorostyrene ( $24 \mu \mathrm{L}, 25 \mathrm{mg}, 0.20 \mathrm{mmol}), 2$-amino-4-methylpyridine (22 $\mathrm{mg}, 0.20 \mathrm{mmol}$ ), $\mathrm{Ru}\left(\mathrm{PEt}_{3}\right)_{3}\left(\mathrm{NTf}_{2}\right)_{2}(20 \mathrm{mg}, 0.010 \mathrm{mmol}, 10 \mathrm{~mol} \%), 1,2-\mathrm{DCB}(60 \mu \mathrm{L})$ and a magnetic stir bar were combined in a one dram vial. The vial threads were wrapped with 1 layer of Teflon tape and the resulting mixture was heated at $120{ }^{\circ} \mathrm{C}$ with stirring for 48 hours. The mixture was allowed to cool, and all of the volatile materials were removed. The crude product was purified by silica gel chromatography, eluting with $30 \%$ ethyl acetate in hexanes. Yield = $26 \mathrm{mg}(57 \%)$. ${ }^{1} \mathrm{H}$ NMR (600 MHz, Chloroform- $d$ ) $\delta 7.92(\mathrm{dd}, J=5.1,0.7 \mathrm{~Hz}, 1 \mathrm{H}), 7.36-7.30(\mathrm{~m}, 2 \mathrm{H}), 7.03-$ $6.95(\mathrm{~m}, 2 \mathrm{H}), 6.40(\mathrm{ddd}, J=5.2,1.4,0.7 \mathrm{~Hz}, 1 \mathrm{H}), 6.00(\mathrm{dt}, J=1.6,0.8 \mathrm{~Hz}, 1 \mathrm{H}), 4.91(\mathrm{~d}, J=6.4$ $\mathrm{Hz}, 1 \mathrm{H}), 4.73(\mathrm{p}, J=6.7 \mathrm{~Hz}, 1 \mathrm{H}), 2.14(\mathrm{~s}, 3 \mathrm{H}), 1.51(\mathrm{~d}, J=6.8 \mathrm{~Hz}, 3 \mathrm{H}) .{ }^{13} \mathrm{C} \mathrm{NMR}(151 \mathrm{MHz}$, $\left.\mathrm{CDCl}_{3}\right) \delta 162.7,161.1,158.2,148.6,148.0,140.7(\mathrm{~d}, J=3.0 \mathrm{~Hz}), 127.4(\mathrm{~d}, J=7.9 \mathrm{~Hz}), 115.5(\mathrm{~d}$, $J=21.4 \mathrm{~Hz}), 114.9,107.1,51.2,24.5,21.3 .{ }^{19} \mathrm{~F} \mathrm{NMR}\left(565 \mathrm{MHz}, \mathrm{CDCl}_{3}\right) \delta-116.18$. GC-MS (EI+): $230(\mathrm{M}), 215\left(\mathrm{M}-\mathrm{CH}_{3}\right)$.<smiles>COc1ccc(C(C)Nc2cc(C)ccn2)cc1</smiles>

\section{N-(1-(4-methoxyphenyl)ethyl)-4-methylpyridin-2-amine (21a)}

4-Methoxystyrene $(27 \mu \mathrm{L}, 27 \mathrm{mg}, 0.20 \mathrm{mmol}), 2$-amino-4-methylpyridine (22 mg, $0.20 \mathrm{mmol}$ ), $\mathrm{Ru}\left(\mathrm{PEt}_{3}\right)_{3}\left(\mathrm{NTf}_{2}\right)_{2}(20 \mathrm{mg}, 0.010 \mathrm{mmol}, 10 \mathrm{~mol} \%), 1,2-\mathrm{DCB}(60 \mu \mathrm{L})$ and a magnetic stir bar were combined in a one dram vial. The vial threads were wrapped with 1 layer of Teflon tape and the resulting mixture was heated at $100{ }^{\circ} \mathrm{C}$ with stirring for 48 hours. The mixture was allowed to cool, and all of the volatile materials were removed. The crude product was purified by silica gel chromatography, eluting with $30 \%$ ethyl acetate in hexanes. Yield $=30 \mathrm{mg}(61 \%)$. ${ }^{1} \mathrm{H}$ NMR $(600 \mathrm{MHz}$, Chloroform- $d$ ) $\delta 7.93(\mathrm{dd}, J=5.1,0.8 \mathrm{~Hz}, 1 \mathrm{H}), 7.32-7.27(\mathrm{~m}, 2 \mathrm{H}), 6.88-$ $6.82(\mathrm{~m}, 2 \mathrm{H}), 6.38(\mathrm{ddd}, J=5.2,1.5,0.7 \mathrm{~Hz}, 1 \mathrm{H}), 6.03(\mathrm{dt}, J=1.6,0.8 \mathrm{~Hz}, 1 \mathrm{H}), 4.91(\mathrm{~d}, J=6.5$ $\mathrm{Hz}, 1 \mathrm{H}), 4.69(\mathrm{p}, J=6.7 \mathrm{~Hz}, 1 \mathrm{H}), 3.78(\mathrm{~s}, 3 \mathrm{H}), 2.14(\mathrm{~d}, J=0.7 \mathrm{~Hz}, 3 \mathrm{H}), 1.51(\mathrm{~d}, J=6.7 \mathrm{~Hz}$, $3 \mathrm{H}) .{ }^{13} \mathrm{C} \mathrm{NMR}\left(151 \mathrm{MHz}, \mathrm{CDCl}_{3}\right) \delta 158.6,158.4,148.5,147.9,137.0,127.0,114.6,114.0$, 107.1, 55.3, 51.3, 24.4, 21.3. GC-MS (EI+): 242 (M), $227\left(\mathrm{M}-\mathrm{CH}_{3}\right)$. 
<smiles>Cc1ccnc(NC(C)c2ccc(C(F)(F)F)cc2)c1</smiles>

\section{4-methyl-N-(1-(4-(trifluoromethyl)phenyl)ethyl)pyridin-2-amine (22a)}

4-Trifluoromethylstyrene ( $30 \mu \mathrm{L}, 34 \mathrm{mg}, 0.20 \mathrm{mmol}$ ), 2-amino-4-methylpyridine (22 $\mathrm{mg}, 0.20$ $\mathrm{mmol}), \mathrm{Ru}\left(\mathrm{PEt}_{3}\right)_{3}\left(\mathrm{NTf}_{2}\right)_{2}(20 \mathrm{mg}, 0.010 \mathrm{mmol}, 10 \mathrm{~mol} \%), 1,2-\mathrm{DCB}(60 \mu \mathrm{L})$ and a magnetic stir bar were combined in a one dram vial. The vial threads were wrapped with 1 layer of Teflon tape and the resulting mixture was heated at $120{ }^{\circ} \mathrm{C}$ with stirring for 48 hours. The mixture was allowed to cool, and all of the volatile materials were removed. The crude product was purified by silica gel chromatography, eluting with $30 \%$ ethyl acetate in hexanes. Yield $=33 \mathrm{mg}(60 \%)$. ${ }^{1} \mathrm{H}$ NMR (600 MHz, Chloroform- $d$ ) $\delta 7.93(\mathrm{~d}, J=5.2 \mathrm{~Hz}, 1 \mathrm{H}), 7.57(\mathrm{~d}, J=8.1 \mathrm{~Hz}, 2 \mathrm{H}), 7.49(\mathrm{~d}$, $J=8.1 \mathrm{~Hz}, 2 \mathrm{H}), 6.44-6.37(\mathrm{~m}, 1 \mathrm{H}), 4.95(\mathrm{~b}, 1 \mathrm{H}), 4.88-4.77(\mathrm{p}, J=6.8 \mathrm{~Hz}, 1 \mathrm{H}), 2.14(\mathrm{~s}, 3 \mathrm{H})$, $1.54(\mathrm{~d}, J=6.8 \mathrm{~Hz}, 3 \mathrm{H}) .{ }^{13} \mathrm{C}$ NMR $\left(151 \mathrm{MHz}, \mathrm{CDCl}_{3}\right) \delta 158.0,149.3,148.7,148.0,129.3(\mathrm{q}, J=$ $32.1 \mathrm{~Hz}), 124.3(\mathrm{q}, J=272.0 \mathrm{~Hz}), 126.3,125.7,115.1,107.2,51.5,24.4,21.3 .{ }^{19} \mathrm{~F}$ NMR $(565$ $\left.\mathrm{MHz}, \mathrm{CDCl}_{3}\right) \delta-62.37$. GC-MS (EI+): $280(\mathrm{M}), 265\left(\mathrm{M}-\mathrm{CH}_{3}\right)$.<smiles>Cc1ccnc(NC(C)c2ccc(Cl)cc2)c1</smiles>

\section{N-(1-(4-chlorophenyl)ethyl)-4-methylpyridin-2-amine (23a)}

4-Chlorostyrene $(24 \mu \mathrm{L}, 28 \mathrm{mg}, 0.20 \mathrm{mmol}), 2$-amino-4-methylpyridine (22 $\mathrm{mg}, 0.20 \mathrm{mmol}$ ), $\mathrm{Ru}\left(\mathrm{PEt}_{3}\right)_{3}\left(\mathrm{NTf}_{2}\right)_{2}(20 \mathrm{mg}, 0.010 \mathrm{mmol}, 10 \mathrm{~mol} \%), 1,2-\mathrm{DCB}(60 \mu \mathrm{L})$ and a magnetic stir bar were combined in a one dram vial. The vial threads were wrapped with 1 layer of Teflon tape and the resulting mixture was heated at $120{ }^{\circ} \mathrm{C}$ with stirring for 48 hours. The mixture was allowed to cool, and all of the volatile materials were removed. The crude product was purified by silica gel chromatography, eluting with $30 \%$ ethyl acetate in hexanes. Yield $=24 \mathrm{mg}(49 \%)$. ${ }^{1} \mathrm{H}$ NMR $(600 \mathrm{MHz}$, Chloroform- $d) \delta 7.92(\mathrm{~d}, J=5.2 \mathrm{~Hz}, 1 \mathrm{H}), 7.35-7.21(\mathrm{~m}, 4 \mathrm{H}), 6.39(\mathrm{dd}, J=$ $5.2,1.4 \mathrm{~Hz}, 1 \mathrm{H}), 5.99(\mathrm{~s}, 1 \mathrm{H}), 4.99(\mathrm{~d}, J=6.4 \mathrm{~Hz}, 1 \mathrm{H}), 4.72(\mathrm{p}, J=6.7 \mathrm{~Hz}, 1 \mathrm{H}), 2.13(\mathrm{~s}, 3 \mathrm{H})$, $1.50(\mathrm{~d}, J=6.8 \mathrm{~Hz}, 3 \mathrm{H}) .{ }^{13} \mathrm{C} \mathrm{NMR}\left(151 \mathrm{MHz}, \mathrm{CDCl}_{3}\right) \delta 158.1,148.6,147.9,143.6,132.6,128.8$, 127.3, 114.9, 107.1, 51.3, 24.4, 21.3. GC-MS (EI+): 248 (M+2), 246 (M), $231\left(\mathrm{M}-\mathrm{CH}_{3}\right)$.<smiles>Cc1ccnc(NC(C)c2ccc(Br)cc2)c1</smiles> 
N-(1-(4-bromophenyl)ethyl)-4-methylpyridin-2-amine (24a)

4-Bromostyrene ( $26 \mu \mathrm{L}, 37 \mathrm{mg}, 0.20 \mathrm{mmol}), 2$-amino-4-methylpyridine (22 $\mathrm{mg}, 0.20 \mathrm{mmol}$ ), $\mathrm{Ru}\left(\mathrm{PEt}_{3}\right)_{3}\left(\mathrm{NTf}_{2}\right)_{2}(20 \mathrm{mg}, 0.010 \mathrm{mmol}, 10 \mathrm{~mol} \%), 1,2-\mathrm{DCB}(60 \mu \mathrm{L})$ and a magnetic stir bar were combined in a one dram vial. The vial threads were wrapped with 1 layer of Teflon tape and the resulting mixture was heated at $120{ }^{\circ} \mathrm{C}$ with stirring for 48 hours. The mixture was allowed to cool, and all of the volatile materials were removed. The crude product was purified by silica gel chromatography, eluting with $20 \%$ ethyl acetate in hexanes. Yield $=27 \mathrm{mg}(46 \%)$. ${ }^{1} \mathrm{H}$ NMR $(500 \mathrm{MHz}$, Chloroform- $d$ ) $\delta 7.92(\mathrm{~d}, J=5.2 \mathrm{~Hz}, 1 \mathrm{H}), 7.47-7.40(\mathrm{~m}, 2 \mathrm{H}), 7.30-7.19$ $(\mathrm{m}, 2 \mathrm{H}), 6.41(\mathrm{dd}, J=5.2,1.4 \mathrm{~Hz}, 1 \mathrm{H}), 5.99(\mathrm{~s}, 1 \mathrm{H}), 4.89(\mathrm{~d}, J=6.2 \mathrm{~Hz}, 1 \mathrm{H}), 4.70(\mathrm{p}, J=6.7 \mathrm{~Hz}$, $1 \mathrm{H}), 2.14(\mathrm{~s}, 3 \mathrm{H}), 1.51(\mathrm{~d}, J=6.8 \mathrm{~Hz}, 3 \mathrm{H}) .{ }^{13} \mathrm{C} \mathrm{NMR}\left(151 \mathrm{MHz}, \mathrm{CDCl}_{3}\right) \delta$ 158.1, 148.6, 148.0, 144.1, 131.8, 127.7, 120.7, 115.0, 107.1, 51.4, 24.4, 21.3. GC-MS (EI+): 292 (M+2), 290 (M), $275\left(\mathrm{M}-\mathrm{CH}_{3}\right)$.<smiles>Cc1ccnc(NC(C)c2ccccc2C)c1</smiles>

\section{4-methyl-N-(1-(o-tolyl)ethyl)pyridin-2-amine (25a)}

2-Methylstyrene ( $26 \mu \mathrm{L}, 24 \mathrm{mg}, 0.20 \mathrm{mmol}), 2$-amino-4-methylpyridine $(22 \mathrm{mg}, 0.20 \mathrm{mmol}$ ), $\mathrm{Ru}\left(\mathrm{PEt}_{3}\right)_{3}\left(\mathrm{NTf}_{2}\right)_{2}(20 \mathrm{mg}, 0.010 \mathrm{mmol}, 10 \mathrm{~mol} \%), 1,2-\mathrm{DCB}(60 \mu \mathrm{L})$ and a magnetic stir bar were combined in a one dram vial. The vial threads were wrapped with 1 layer of Teflon tape and the resulting mixture was heated at $120{ }^{\circ} \mathrm{C}$ with stirring for 48 hours. The mixture was allowed to cool, and all of the volatile materials were removed. The crude product was purified by silica gel chromatography, eluting with $30 \%$ ethyl acetate in hexanes. Yield $=18 \mathrm{mg}(40 \%) .{ }^{1} \mathrm{H}$ NMR $(600$ MHz, Chloroform- $d$ ) $\delta 7.93(\mathrm{~d}, J=5.1 \mathrm{~Hz}, 1 \mathrm{H}), 7.42-7.38(\mathrm{~m}, 1 \mathrm{H}), 7.20-7.11(\mathrm{~m}, 3 \mathrm{H}), 6.38$ $(\mathrm{dd}, J=5.2,1.4 \mathrm{~Hz}, 1 \mathrm{H}), 5.92(\mathrm{~d}, J=1.5 \mathrm{~Hz}, 1 \mathrm{H}), 4.97-4.89(\mathrm{~m}, 1 \mathrm{H}), 4.82(\mathrm{~d}, J=6.5 \mathrm{~Hz}, 1 \mathrm{H})$, $2.44(\mathrm{~s}, 3 \mathrm{H}), 2.13(\mathrm{~s}, 3 \mathrm{H}), 1.50(\mathrm{~d}, J=6.6 \mathrm{~Hz}, 3 \mathrm{H}) \cdot{ }^{13} \mathrm{C} \mathrm{NMR}\left(151 \mathrm{MHz}, \mathrm{CDCl}_{3}\right) \delta 158.3,148.5$, 148.0, 142.6, 135.0, 130.7, 126.9, 126.6, 124.8, 114.7, 106.8, 48.3, 22.6, 21.4, 19.2. GC-MS (EI+): $226(\mathrm{M}), 211\left(\mathrm{M}-\mathrm{CH}_{3}\right)$.<smiles>COc1ccc(C(C)Nc2ccc(C)cn2)cc1OC</smiles>

$N$-(1-(3,4-dimethoxyphenyl)ethyl)-5-methylpyridine-2-amine (26a)

3,4-dimethoxy-vinylbenzene (33 mg, $0.20 \mathrm{mmol}$ ), 2-amino-5-methylpyridine (22 $\mathrm{mg}, 0.20$ $\mathrm{mmol}), \mathrm{Ru}\left(\mathrm{PEt}_{3}\right)_{3}\left(\mathrm{NTf}_{2}\right)_{2}(20 \mathrm{mg}, 0.010 \mathrm{mmol}, 10 \mathrm{~mol} \%), 1,2-\mathrm{DCB}(60 \mu \mathrm{L})$ and a magnetic stir bar were combined in a one dram vial. The vial threads were wrapped with 1 layer of Teflon tape and the resulting mixture was heated at $100{ }^{\circ} \mathrm{C}$ with stirring for 48 hours. The reaction was allowed to cool, and all of the volatile materials were evaporated. The crude product was purified by silica gel chromatography, eluting with $30 \%$ ethyl acetate in hexanes. Yield $=35 \mathrm{mg}(64 \%)$. 
${ }^{1} \mathrm{H}$ NMR (600 MHz, chloroform- $\left.d\right) \delta 7.90(\mathrm{~s}, 1 \mathrm{H}), 7.16(\mathrm{~d}, \mathrm{~J}=10.0 \mathrm{~Hz}, 1 \mathrm{H}), 6.90(\mathrm{~d}, \mathrm{~J}=10.7 \mathrm{~Hz}$, $2 \mathrm{H}), 6.81(\mathrm{~d}, \mathrm{~J}=8.1 \mathrm{~Hz}, 1 \mathrm{H}), 6.14(\mathrm{~d}, \mathrm{~J}=8.4 \mathrm{~Hz}, 1 \mathrm{H}), 4.76(\mathrm{~d}, \mathrm{~J}=5.5 \mathrm{~Hz}, 1 \mathrm{H}), 4.61(\mathrm{p}, \mathrm{J}=6.5$ $\mathrm{Hz}, 1 \mathrm{H}), 3.85(\mathrm{~s}, 6 \mathrm{H}), 2.14(\mathrm{~s}, 3 \mathrm{H}), 1.52(\mathrm{~d}, \mathrm{~J}=6.7 \mathrm{~Hz}, 3 \mathrm{H}) .{ }^{13} \mathrm{C}$ NMR (151 MHz, chloroform- $d$ ) $\delta 156.1,149.1,147.8,147.4,138.5,137.4,121.8,117.7,111.1,109.0,106.4,55.8,55.8,51.9$, 24.4, 17.3. GC-MS (EI+): 272 (M), $267\left(\mathrm{M}-\mathrm{CH}_{3}\right), 165$ (M-[p-Me-aminopyridine] $\left.{ }^{\circ}\right)$.<smiles>Cc1ccc(NC(C)c2ccc3ccccc3c2)nc1</smiles>

\section{$N$-(ethyl-naphthalene-2-yl)-5-methylpyridin-2-amine (27a)}

Vinyl naphthalene (31 mg, $0.20 \mathrm{mmol}$ ), 2-amino-5-methylpyridine (22 $\mathrm{mg}, 0.20 \mathrm{mmol}$ ), $\mathrm{Ru}\left(\mathrm{PEt}_{3}\right)_{3}\left(\mathrm{NTf}_{2}\right)_{2}(20 \mathrm{mg}, 0.010 \mathrm{mmol}, 10 \mathrm{~mol} \%), 1,2-\mathrm{DCB}(60 \mu \mathrm{L})$ and a magnetic stir bar were combined in a one dram vial. The vial threads were wrapped with 1 layer of Teflon tape and the resulting mixture was heated at $100{ }^{\circ} \mathrm{C}$ with stirring for 48 hours. The reaction was allowed to cool, and all of the volatile materials were evaporated. The crude product was purified by silica gel chromatography, eluting with $40 \%$ ethyl acetate in hexanes. Yield $=34 \mathrm{mg}(65 \%)$. ${ }^{1} \mathrm{H}$ NMR (600 MHz, chloroform- $d$ ) $\delta 7.93-7.90(\mathrm{~m}, 1 \mathrm{H}), 7.84-7.77(\mathrm{~m}, 4 \mathrm{H}), 7.50$ (dd, $J=8.5$, $1.7 \mathrm{~Hz}, 1 \mathrm{H}), 7.45(\mathrm{pd}, J=6.8,1.5 \mathrm{~Hz}, 2 \mathrm{H}), 7.11(\mathrm{dd}, J=8.4,2.4 \mathrm{~Hz}, 1 \mathrm{H}), 6.16(\mathrm{~d}, J=8.5 \mathrm{~Hz}$, $1 \mathrm{H}), 4.95(\mathrm{~d}, J=5.9 \mathrm{~Hz}, 1 \mathrm{H}), 4.83(\mathrm{p}, J=6.6 \mathrm{~Hz}, 1 \mathrm{H}), 2.12(\mathrm{~s}, 3 \mathrm{H}), 1.61(\mathrm{~d}, J=6.8 \mathrm{~Hz}, 3 \mathrm{H}) .{ }^{13} \mathrm{C}$ NMR $(151 \mathrm{MHz}$, chloroform- $d$ ) $\delta 156.4,148.0,142.5,138.5,133.6,132.8,128.6,127.9,127.7$, 126.1, 125.6, 124.5, 124.3, 122.0, 106.5, 52.4, 24.6, 17.4. GC-MS (EI+): 262 (M), 155 (M-[pMe-aminopyridine] ${ }^{\circ}$ ).

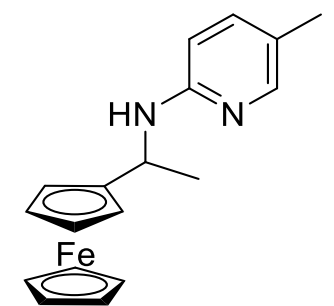

\section{$\mathrm{N}$-(ethylferrocene-2-yl)-5-methylpyridin-2-amine (28a)}

Vinyl ferrocene (42 mg, $0.20 \mathrm{mmol}$ ), 2-amino-5-methylpyridine (22 $\mathrm{mg}, 0.20 \mathrm{mmol}$ ), $\mathrm{Ru}\left(\mathrm{PEt}_{3}\right)_{3}\left(\mathrm{NTf}_{2}\right)_{2}(20 \mathrm{mg}, 0.010 \mathrm{mmol}, 10 \mathrm{~mol} \%), 1,2-\mathrm{DCB}(60 \mu \mathrm{L})$ and a magnetic stir bar were combined in a one dram vial. The vial threads were wrapped with 1 layer of Teflon tape and the resulting mixture was heated at $100{ }^{\circ} \mathrm{C}$ with stirring for 48 hours. The reaction was allowed to cool, and all of the volatile materials were evaporated. The crude product was purified by silica gel chromatography, eluting with $40 \%$ ethyl acetate in hexanes. Yield $=39 \mathrm{mg}(61 \%)$. ${ }^{1} \mathrm{H}$ NMR (600 MHz, chloroform- $\left.d\right) \delta 7.95(\mathrm{~s}, 1 \mathrm{H}), 7.27-7.24(\mathrm{~m}, J=2.0 \mathrm{~Hz}, 1 \mathrm{H}), 6.34(\mathrm{~d}, J=$ $8.4 \mathrm{~Hz}, 1 \mathrm{H}), 4.66(\mathrm{~m}, 1 \mathrm{H}), 4.55(\mathrm{~d}, J=8.2 \mathrm{~Hz}, 1 \mathrm{H}), 4.20(\mathrm{~s}, 5 \mathrm{H}), 4.18(\mathrm{~m}, 2 \mathrm{H}), 4.13(\mathrm{dt}, J=7.7$, $1.7 \mathrm{~Hz}, 2 \mathrm{H}), 2.18(\mathrm{~s}, 3 \mathrm{H}), 1.52(\mathrm{~d}, J=6.5 \mathrm{~Hz}, 3 \mathrm{H}) .{ }^{13} \mathrm{C}$ NMR $(151 \mathrm{MHz}$, chloroform-d) $\delta 156.4$, 147.9, 138.5, 121.5, 107.0, 93.3, 68.5, 67.8, 67.6, 67.0, 66.1, 45.7, 21.3, 17.5. GCMS: (EI+): 320 (M), $305\left(\mathrm{M}^{\left.-\mathrm{CH}_{3}\right),} 213\right.$ (M-[p-Me-aminopyridine] $\left.{ }^{\circ}\right)$.

\section{Removal of Pyridyl Group from the Hydroamination Product}




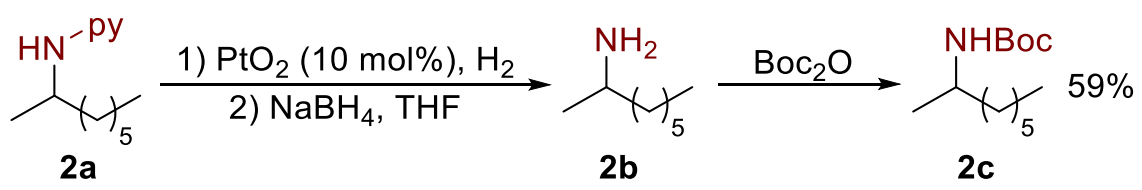

5-methyl- $N$-(octan-2-yl)pyridine-2-amine $2 \mathbf{a}(44 \mathrm{mg}, 0.20 \mathrm{mmol}), \mathrm{HCl}$ in dioxane $(4.0 \mathrm{M}, 0.10$ $\mathrm{mL}, 2.0$ equiv), ethanol $(2.0 \mathrm{~mL})$ and a stir bar were combined in a reaction tube. The tube was capped with a septum-cap and stirred for 15 minutes. $\mathrm{PtO}_{2}(4.6 \mathrm{mg}, 10 \mathrm{~mol} \%)$ was added to the tube and the tube was capped and sealed. The tube was purged with $\mathrm{H}_{2}$ for 10 minutes using a hydrogen balloon and placed in a cold bath set at $0{ }^{\circ} \mathrm{C}$. After 15 hours, the mixture was filtered through celite and the solvent was removed in vacuo. The residue was dissolved in $1.0 \mathrm{~mL}$ of $\mathrm{THF}$ and $0.2 \mathrm{~mL}$ of EtOH and placed in in a cold bath set at $0{ }^{\circ} \mathrm{C}$. Sodium borohydride $(76 \mathrm{mg}$, $2.0 \mathrm{mmol}, 10$ equiv) was added to the mixture and the mixture was allowed to stir for 2 hours. Water $(10 \mathrm{~mL})$ was added to the mixture and the mixture was extracted with DCM $(10 \mathrm{~mL})$ three times. The combined organic layer was dried over anhydrous $\mathrm{Na}_{2} \mathrm{SO}_{4}$. The solvent was then removed in vacuo and the residue was dissolved in $2.0 \mathrm{~mL}$ of THF. $\mathrm{Boc}_{2} \mathrm{O}(87 \mathrm{mg}, 0.40 \mathrm{mmol}$, 2.0 equiv) and 4-Dimethylaminopyridine ( $2.4 \mathrm{mg}, 0.020 \mathrm{mmol}, 0.10$ equiv) was added to the mixture and the mixture was stirred overnight. The solvent was removed and the crude Bocprotected amine product 2c was purified by column chromatography, eluting with $10 \%$ ethyl acetate in hexanes. Yield $=27 \mathrm{mg}(59 \%)$. ${ }^{1} \mathrm{H}$ NMR $\left(600 \mathrm{MHz}, \mathrm{CDCl}_{3}\right) \delta 4.32(\mathrm{~s}, 1 \mathrm{H}), 3.64(\mathrm{~s}$, $1 \mathrm{H}), 1.46(\mathrm{~s}, 9 \mathrm{H}), 1.37-1.42(\mathrm{~m}, 2 \mathrm{H}), 1.35-1.23(\mathrm{~m}, 8 \mathrm{H}), 1.11(\mathrm{~d}, J=6.6 \mathrm{~Hz}, 3 \mathrm{H}), 0.89(\mathrm{t}, J=$ $6.8 \mathrm{~Hz}, 3 \mathrm{H})$.

\section{Determination of the Catalyst Resting State}

\section{Initial Investigation of the Catalyst Resting State}

$\mathrm{Ru}\left(\mathrm{PEt}_{3}\right)_{3}\left(\mathrm{NTf}_{2}\right)_{2}(\mathbf{R u}-\mathbf{1})(10 \mathrm{mg}, 0.010 \mathrm{mmol})$ were combined with $\mathrm{D}_{4}-\mathrm{DCB}(0.50 \mathrm{~mL})$ and appropriate amount of 2-amino-4-methylpyridine (1b) and dodecane in a J-young tube. The tube was heated at $80{ }^{\circ} \mathrm{C}$ for 4 hours and a ${ }^{31} \mathrm{P}$ NMR spectrum of the mixture was taken at $80{ }^{\circ} \mathrm{C}$.

\section{Independent Synthesis of the Catalyst Resting State}

$\operatorname{Ru}\left(\text { PEt }_{3}\right)_{3}$ (amine)(NTf $)_{2}$ (29)

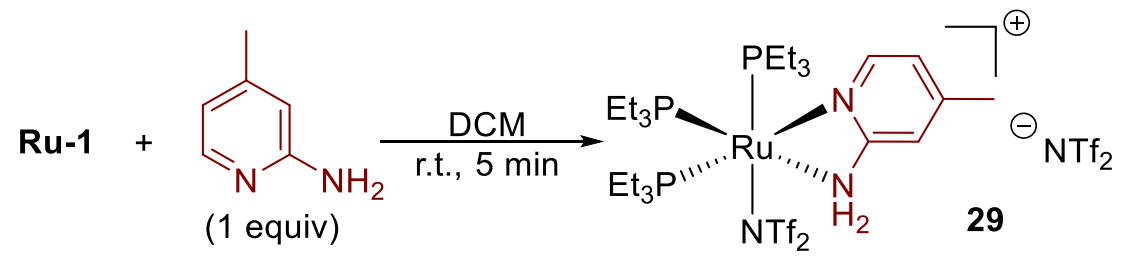

$\mathrm{Ru}\left(\mathrm{PEt}_{3}\right)_{3}\left(\mathrm{NTf}_{2}\right)_{2}(\mathbf{R u}-\mathbf{1})(51 \mathrm{mg}, 0.050 \mathrm{mmol}), 2$-amino-5-methylpyridine (5.4 $\left.\mathrm{mg}, 0.050 \mathrm{mmol}\right)$ and DCM $(2.0 \mathrm{~mL})$ were combined in a $20-\mathrm{mL}$ vial and stirred at room temperature for 5 minutes. The mixture turned bright yellow. The solvent was removed under vacuum and the resulting yellow solid was washed with cold diethyl ether three times. Single crystals suitable for $\mathrm{x}$-ray crystallography analysis was obtained by cooling a $\mathrm{DCM} /{ }^{i} \operatorname{Pr}_{2} \mathrm{O}$ saturated solution of $\mathrm{Ru}\left(\mathrm{PEt}_{3}\right)_{3}$ (amine) $\left(\mathrm{NTf}_{2}\right)_{2}(\mathbf{2 9})$. Yield $=46 \mathrm{mg}(82 \%) .{ }^{1} \mathrm{H}$ NMR $(600 \mathrm{MHz}$, Methylene Chloride$\left.d_{2}\right) \delta 8.16(\mathrm{~s}, 1 \mathrm{H}), 7.84(\mathrm{~d}, J=8.2 \mathrm{~Hz}, 1 \mathrm{H}), 7.28(\mathrm{~d}, J=8.2 \mathrm{~Hz}, 1 \mathrm{H}), 5.14(\mathrm{~s}, 2 \mathrm{H}), 2.44(\mathrm{~s}, 3 \mathrm{H})$, 
$2.00-1.80(\mathrm{~m}, 18 \mathrm{H}), 1.15(\mathrm{~m}, 24 \mathrm{H}) .{ }^{31} \mathrm{P}$ NMR $\left(162 \mathrm{MHz}\right.$, Methylene Chloride- $\left.d_{2}\right) \delta 38.33 .{ }^{19} \mathrm{~F}$ NMR (376 MHz, Methylene Chloride- $d_{2}$ ) $\delta-78.19$. The identity of the product was confirmed by x-ray crystallography. Anal. Calc'd C:28.64 H:4.44 N:4.99 Found C:28.51 H:4.46 N:4.61.

$\operatorname{Ru}\left(\text { PEt }_{3}\right)_{3}\left(\right.$ amido) $\left(\right.$ NTf $\left._{2}\right)(30)$
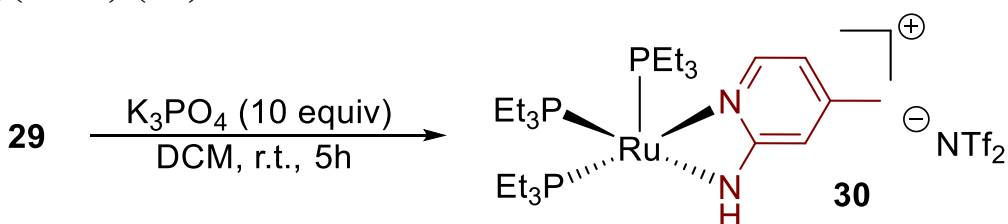

$\mathrm{Ru}\left(\mathrm{PEt}_{3}\right)_{3}($ amine $)\left(\mathrm{NTf}_{2}\right)_{2}(\mathbf{2 9})(56 \mathrm{mg}, 0.050 \mathrm{mmol})$, anhydrous $\mathrm{K}_{3} \mathrm{PO}_{4}(0.11 \mathrm{~g}, 0.50 \mathrm{mmol}, 10$ equiv) and DCM $(3.0 \mathrm{~mL})$ were combined in a $20-\mathrm{mL}$ vial and stirred at room temperature for 5 hours. The black mixture was then filtered, and the solvent was removed under vacuum. The resulting black oil was triturated with cold ${ }^{i} \mathrm{Pr}_{2} \mathrm{O}$ three times to afford black powder. Single crystals suitable for $\mathrm{x}$-ray crystallography analysis was obtained by cooling a $\mathrm{DCM} / \mathrm{Pr}_{2} \mathrm{O}$ saturated solution of $\mathrm{Ru}\left(\mathrm{PEt}_{3}\right)_{3}\left(\right.$ amido) $\left(\mathrm{NTf}_{2}\right)(\mathbf{3 0})$. Yield $=36 \mathrm{mg}(85 \%) .{ }^{1} \mathrm{H}$ NMR $(600 \mathrm{MHz}$, Methylene Chloride- $\left.d_{2}\right) \delta 7.34(\mathrm{~d}, J=5.6 \mathrm{~Hz}, 1 \mathrm{H}), 6.06(\mathrm{~d}, J=5.6 \mathrm{~Hz}, 1 \mathrm{H}), 5.56(\mathrm{~s}, 1 \mathrm{H}), 4.64(\mathrm{~s}$, $1 \mathrm{H}), 2.05(\mathrm{~s}, 3 \mathrm{H}), 1.79(\mathrm{~m}, 18 \mathrm{H}), 1.17(\mathrm{~m}, 24 \mathrm{H}) .{ }^{31} \mathrm{P}$ NMR $\left(243 \mathrm{MHz}\right.$, Methylene Chloride- $\left.d_{2}\right) \delta$ 51.95. ${ }^{19} \mathrm{~F}$ NMR (376 MHz, Methylene Chloride- $d_{2}$ ) $\delta-78.76$. The identity of the product was confirmed by x-ray crystallography. Anal. Calc'd C:36.60 H:5.94 N:5.09 Found C:36.87 H:6.11 $\mathrm{N}: 5.03$.

\section{Examination of the kinetic Competence of $\operatorname{Ru}\left(\mathrm{PEt}_{3}\right)_{3}\left(\operatorname{amido}_{(}\right)\left(\mathrm{NTf}_{2}\right)$}

1-Dodecene (44 $\mu \mathrm{L}, 34 \mathrm{mg}, 0.20 \mathrm{mmol}$ ), 2-amino-4-methylpyridine (22 $\mathrm{mg}, 0.20 \mathrm{mmol}$ ), and $\mathrm{Ru}\left(\mathrm{PEt}_{3}\right)_{3}(\mathrm{amido})\left(\mathrm{NTf}_{2}\right)(\mathbf{3 0})(8.4 \mathrm{mg}, 0.010 \mathrm{mmol}, 5.0 \mathrm{~mol} \%)$ were combined with DCB (60 $\mu \mathrm{L}$ ) and a magnetic stir bar in a one dram vial. The vial was capped, and the resulting mixture was heated at $80{ }^{\circ} \mathrm{C}$ with stirring for 48 hours. The mixture was allowed to cool to room temperature and an NMR yield (65\%) of this reaction was obtained using 1,3,5trimethoxybenzene as the internal standard.

\section{Variable Temperature NMR Spectroscopy Analysis of the Mixture of $\operatorname{Ru}\left(\mathrm{PEt}_{3}\right)_{3}$ (amido)(NTf 2 ) and 2-amino-4-methylpyridine}

$\mathrm{Ru}\left(\mathrm{PEt}_{3}\right)_{3}$ (amido)( $\left.\mathrm{NTf}_{2}\right)(30)(8.4 \mathrm{mg}, 0.010 \mathrm{mmol})$ and 2-amino-4-methylpyridine (22 $\mathrm{mg}, 0.20$ $\mathrm{mmol})$ and were combined with $\mathrm{D}_{4}-\mathrm{DCB}(0.50 \mathrm{~mL})$ in a J-young tube. The mixture turned black and ${ }^{1} \mathrm{H}$ (Figure S1) and ${ }^{31} \mathrm{P}$ NMR spectra of the mixture were taken at $80{ }^{\circ} \mathrm{C}, 60{ }^{\circ} \mathrm{C}, 40{ }^{\circ} \mathrm{C}$ and 25 ${ }^{\circ} \mathrm{C} . \mathrm{Ru}\left(\mathrm{PEt}_{3}\right)_{3}$ (amido)( $\left.\mathrm{NTf}_{2}\right)(30)(8.4 \mathrm{mg}, 0.010 \mathrm{mmol})$ and 2-amino-4-methylpyridine $(22 \mathrm{mg}$, $0.20 \mathrm{mmol})$ and were combined with $\mathrm{D}_{2}-\mathrm{DCM}(0.50 \mathrm{~mL})$ in a J-young tube. The mixture turned black and ${ }^{31} \mathrm{P}$ NMR spectra of the mixture was taken at $0{ }^{\circ} \mathrm{C},-20{ }^{\circ} \mathrm{C}$, and $-40{ }^{\circ} \mathrm{C}$. 

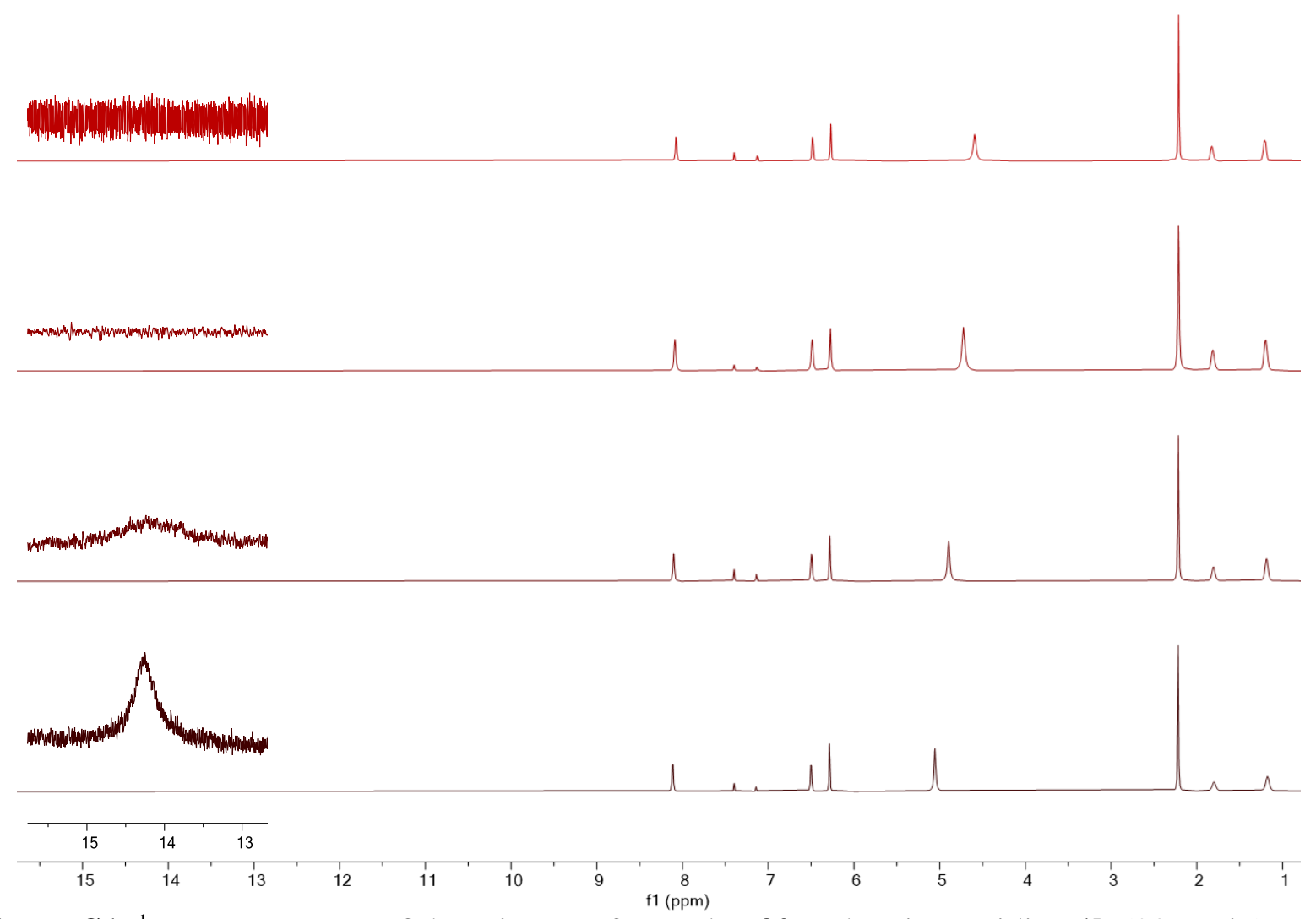

Figure S1. ${ }^{1} \mathrm{H}$ NMR spectra of the mixture of complex $\mathbf{3 0}$ and aminopyridine $\mathbf{1 b}$ (20 equiv) at 80 ${ }^{\circ} \mathrm{C}, 60{ }^{\circ} \mathrm{C}, 40{ }^{\circ} \mathrm{C}$ and $25^{\circ} \mathrm{C}$ (from top to bottom).

\section{Kinetic Studies of Catalytic Hydroamination}

All samples for kinetic experiments were prepared and analyzed according to the following procedures. In a nitrogen-filled glovebox, an appropriate amount of vinylcyclohexane, 2-amino4-methylpyridine, $\mathrm{Ru}\left(\mathrm{PEt}_{3}\right)_{3}\left(\mathrm{NTf}_{2}\right)_{2}$ and 1,3,5-trimethoxybenzene (internal standard) were mixed in a $1.0 \mathrm{~mL}$ volumetric flask. Then, 1,2-dichlorobenzene was added to prepare a $1.0 \mathrm{~mL}$ solution. The mixture was then transferred into a Schlenk tube equipped with a magnetic stir bar. A $t_{0}$ aliquot was then taken and the tube was sealed and heated in an oil bath at $120{ }^{\circ} \mathrm{C}$ outside the glovebox. The Schlenk tube was removed from the oil bath after a certain amount of time of heating and rapidly cooled. The mixture was then taken into the glovebox, and a $50 \mu \mathrm{L}$ aliquot was removed from the mixture. The tube was resealed and returned to the oil bath. The aliquots were analyzed by ${ }^{1} \mathrm{H}$ NMR spectroscopy and concentrations were determined by integration relative to the 1,3,5-trimethoxybenzene internal standard. Initial reaction rates were subsequently determined by measuring product formation at low conversion $(<10 \%)$.

\section{Order in vinylcyclohexane (Figure S2-S5)}




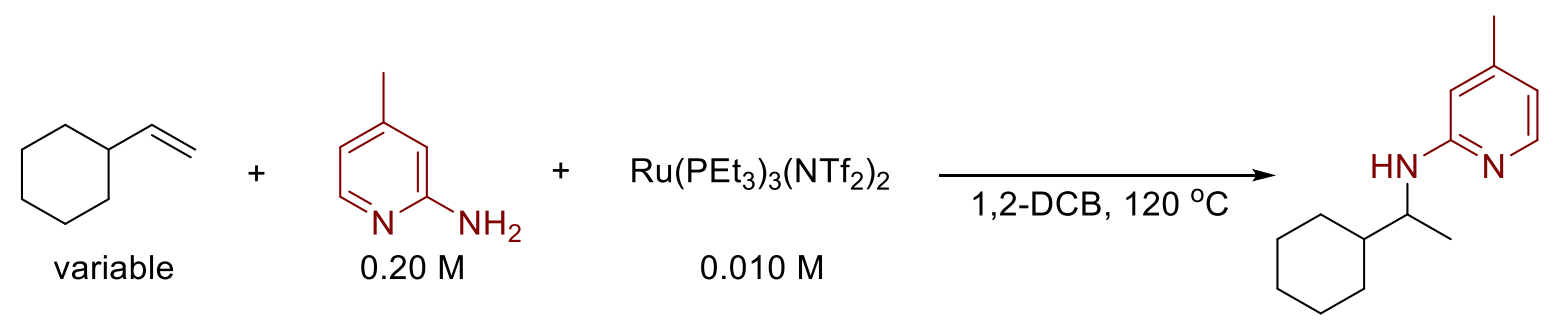

[prod] vs. time: [vinylcyclohexane] $=0.20 \mathrm{M}$

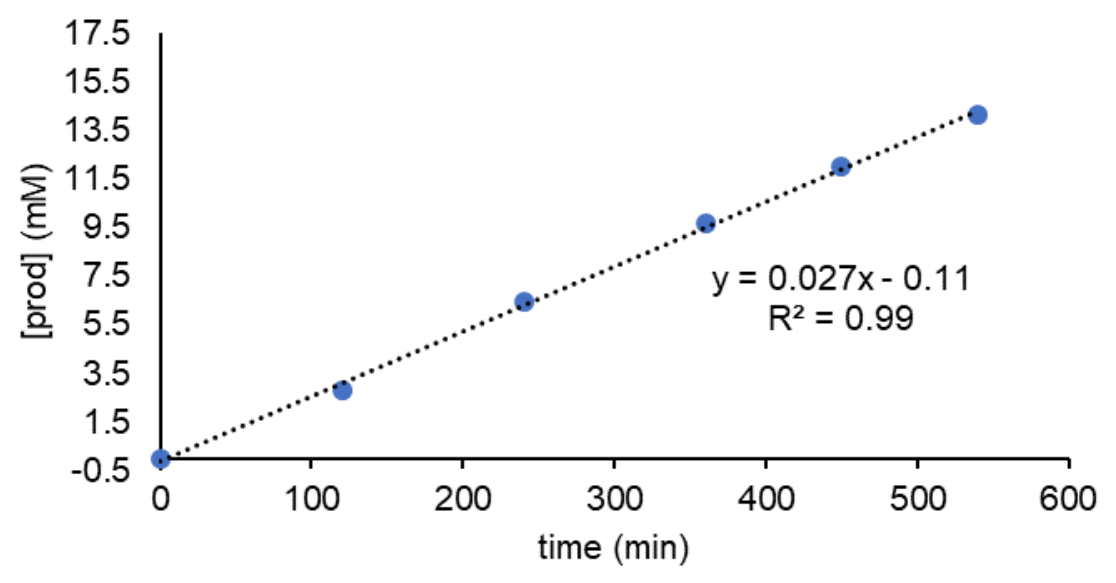

Figure S2. Rate of product formation as a function of time when [vinylcyclohexane] $=0.20 \mathrm{M}$.

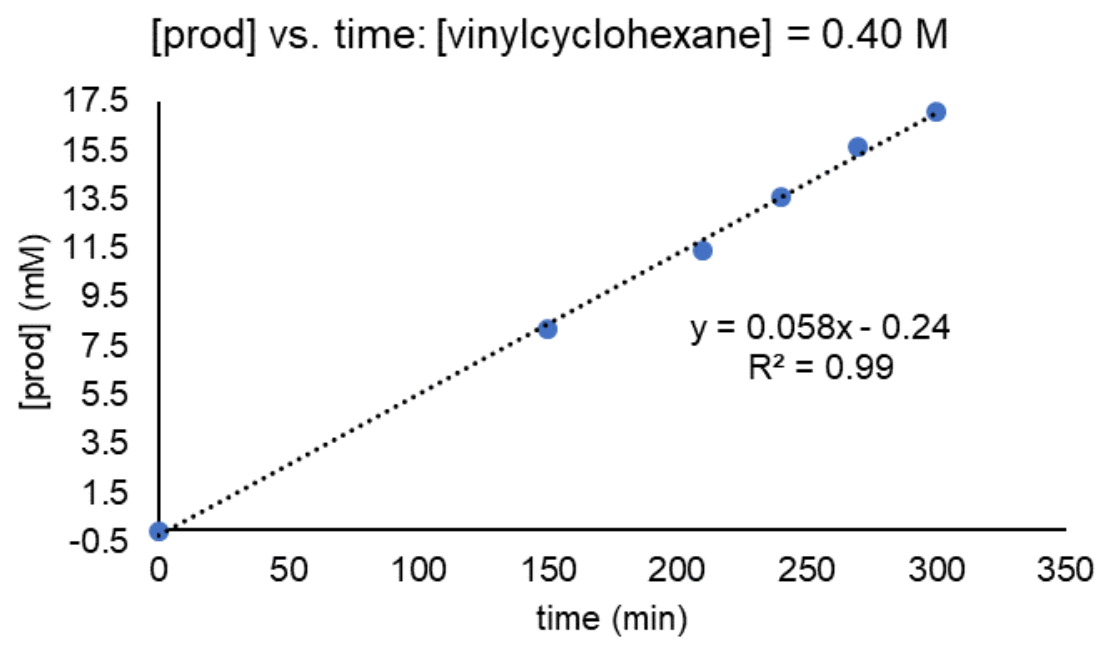

Figure S3. Rate of product formation as a function of time when [vinylcyclohexane] $=0.40 \mathrm{M}$. 


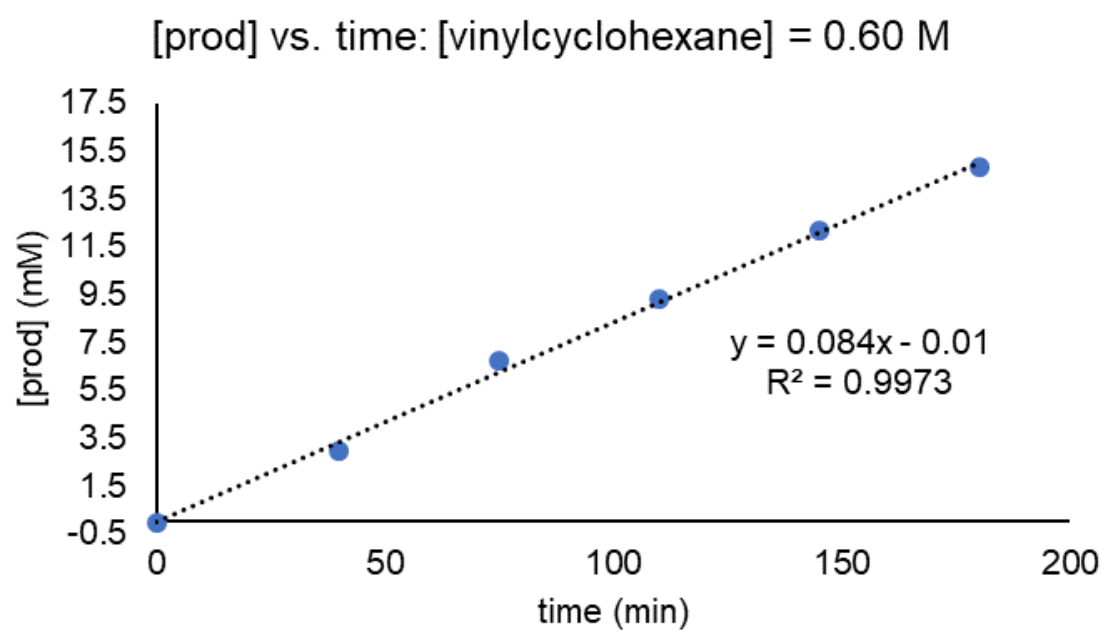

Figure S4. Rate of product formation as a function of time when [vinylcyclohexane] =0.60 M.

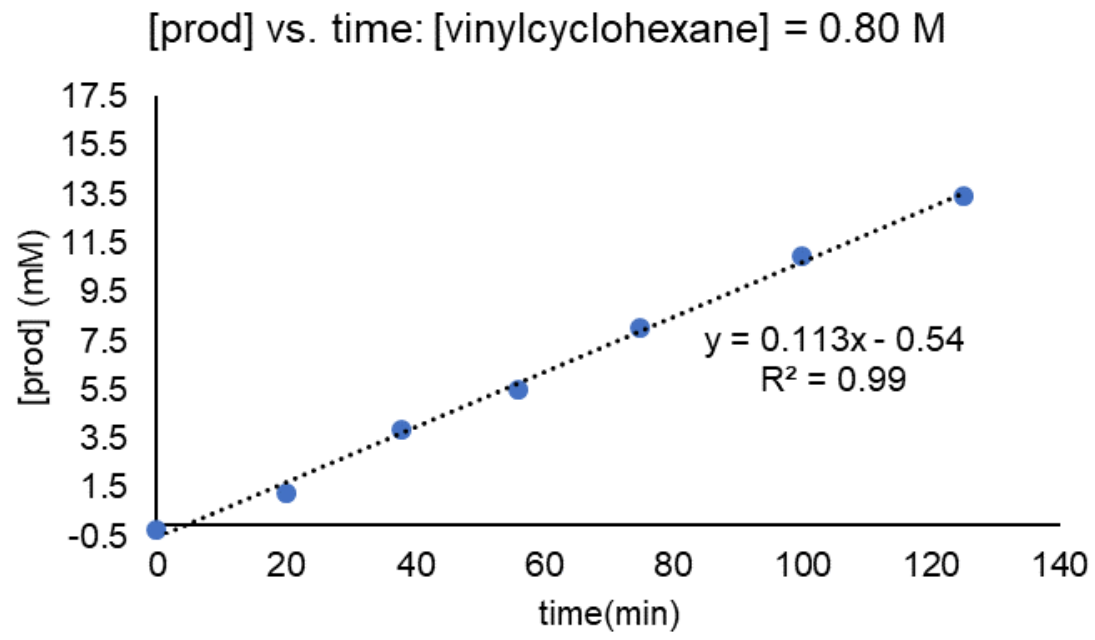

Figure S5. Rate of product formation as a function of time when [vinylcyclohexane] $=0.80 \mathrm{M}$.

\section{Order in 2-amino-4-methylpyridine (1b) (Figure S6-S9)}<smiles>C=CC1CCCCC1</smiles> 


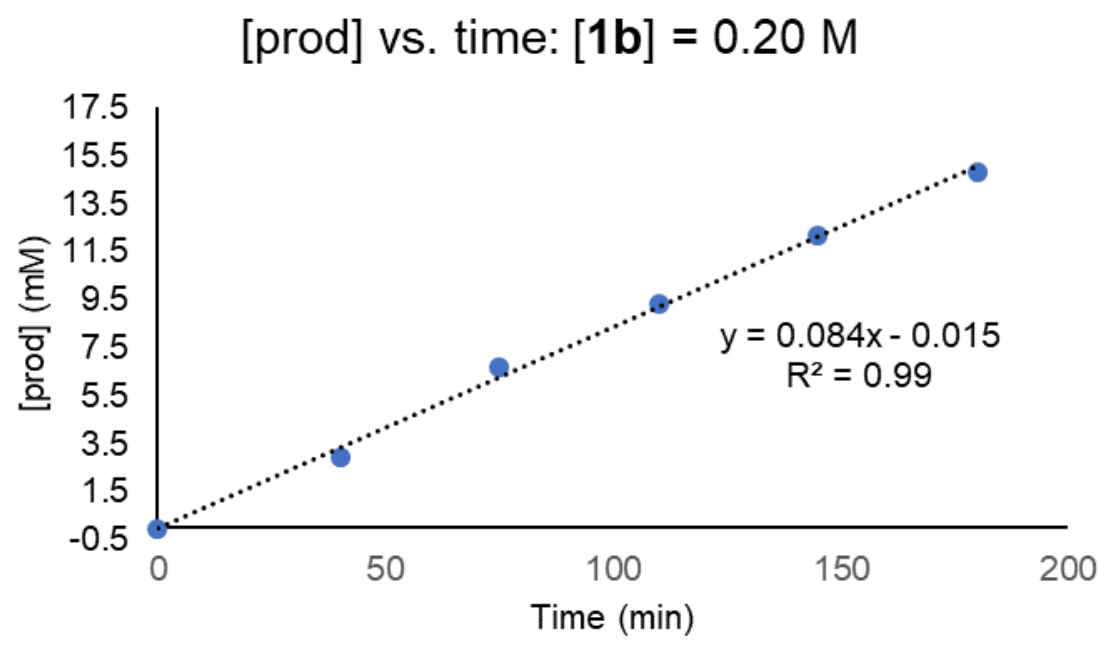

Figure S6. Rate of product formation as a function of time when $[\mathbf{1 b}]=0.20 \mathrm{M}$.

[prod] vs. time: $[1 \mathrm{~b}]=0.30 \mathrm{M}$

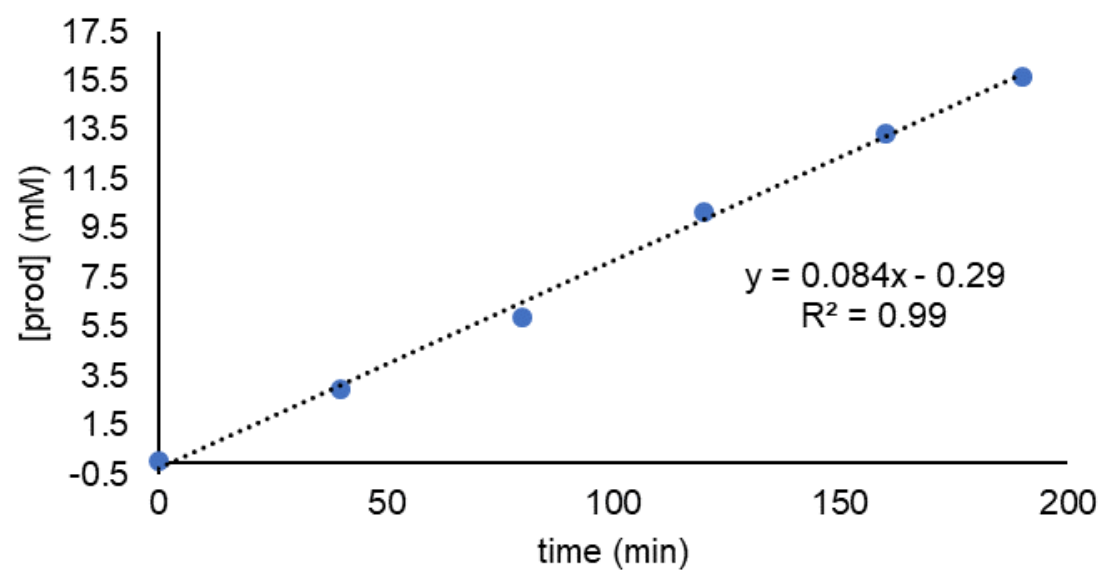

Figure S7. Rate of product formation as a function of time when $[\mathbf{1 b}]=0.30 \mathrm{M}$. 
[prod] vs. time: $[\mathbf{1 b}]=0.40 \mathrm{M}$

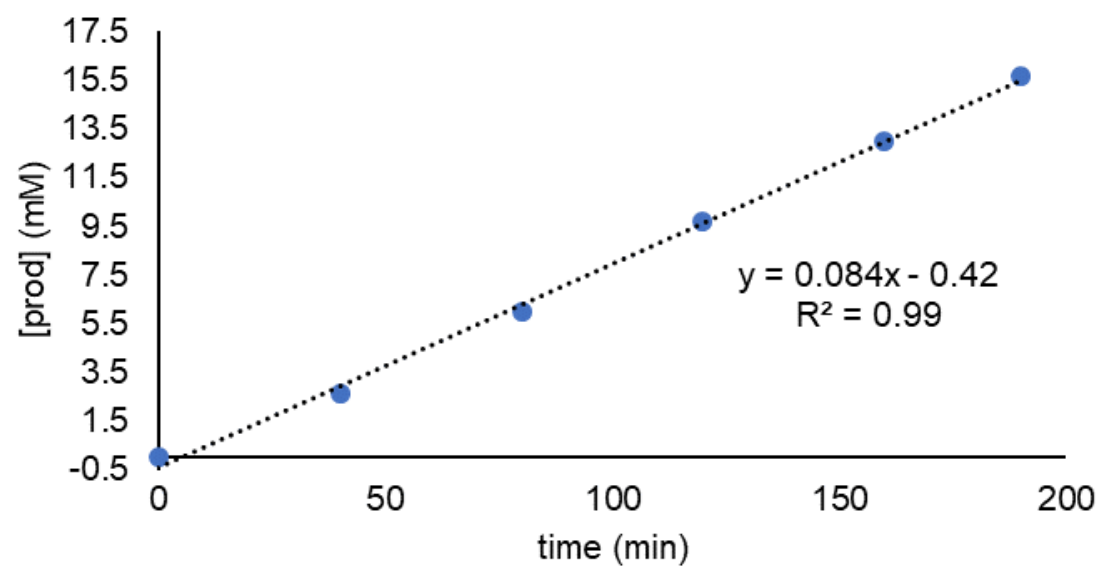

Figure S8. Rate of product formation as a function of time when $[\mathbf{1 b}]=0.40 \mathrm{M}$.

[prod] vs. time: $[\mathbf{1 b}]=0.50 \mathrm{M}$

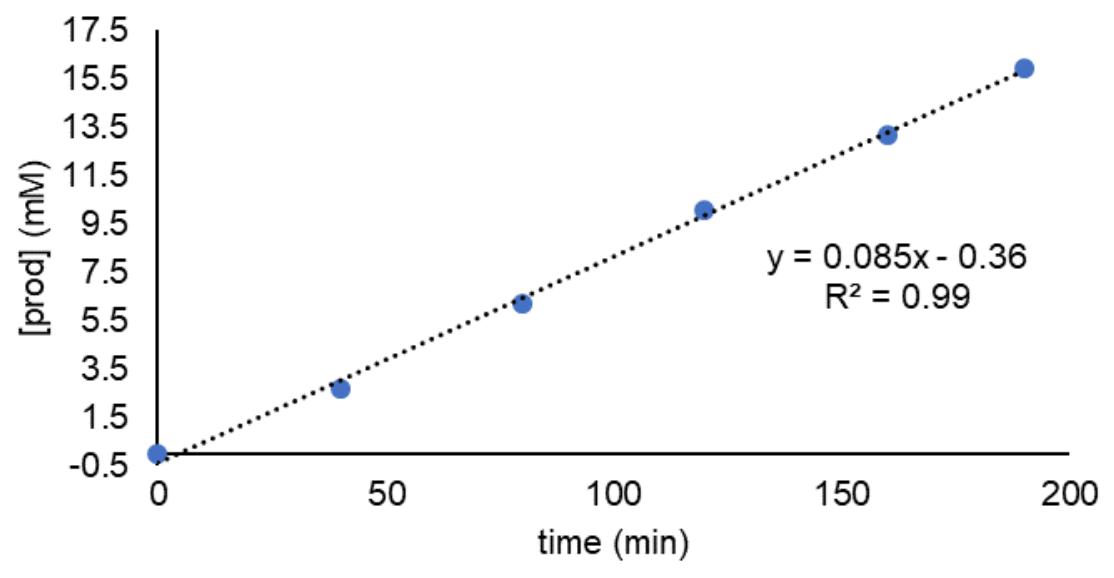

Figure S9. Rate of product formation as a function of time when $[\mathbf{1 b}]=0.50 \mathrm{M}$.

\section{Order in $\operatorname{Ru}\left(\mathbf{P E t}_{3}\right)_{3}\left(\mathrm{NTf}_{2}\right)_{2}($ Ru-1) (Figure S10-S13)}

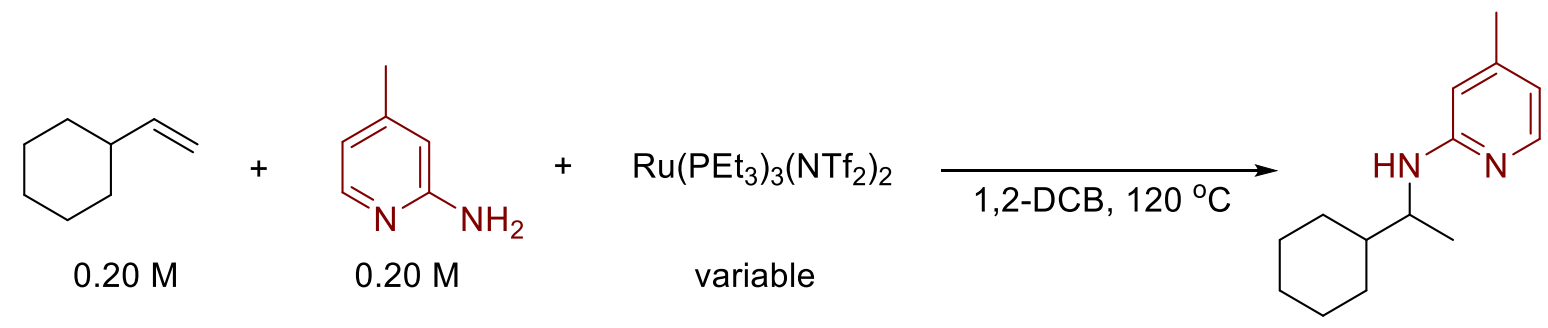




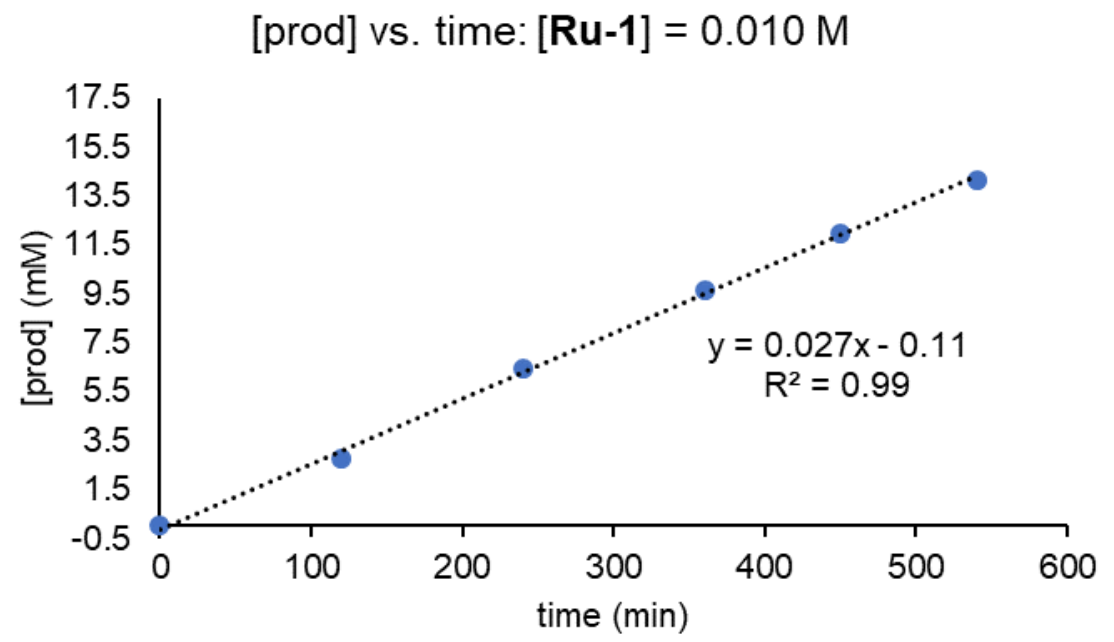

Figure S10. Rate of product formation as a function of time when $[\mathbf{R u}-\mathbf{1}]=0.010 \mathrm{M}$.

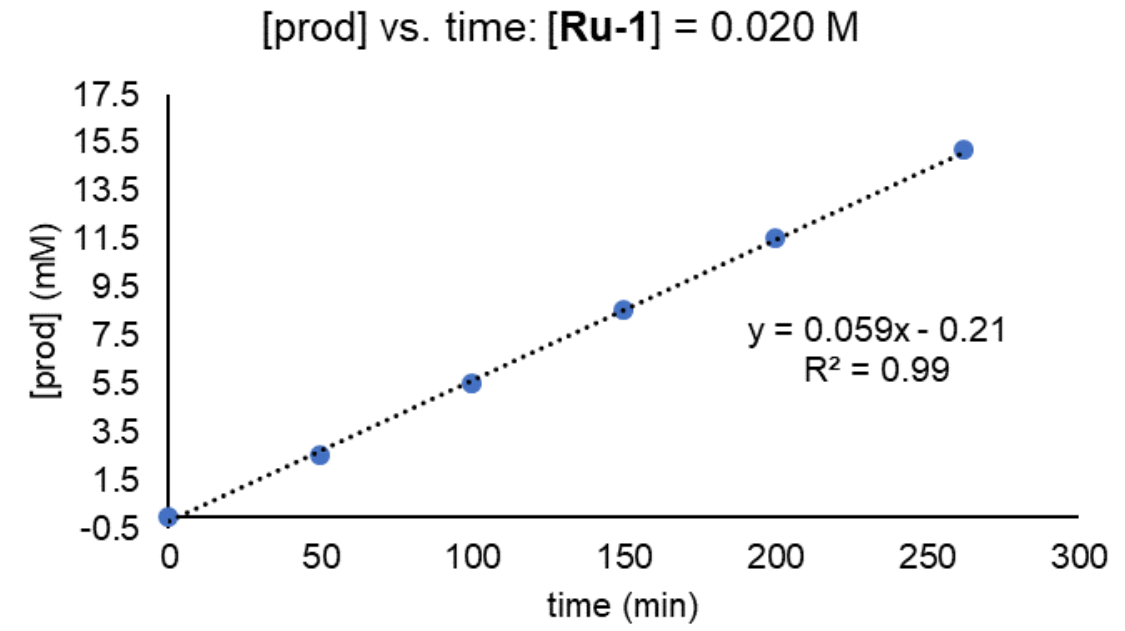

Figure S11. Rate of product formation as a function of time when $[\mathbf{R u - 1}]=0.020 \mathrm{M}$. 


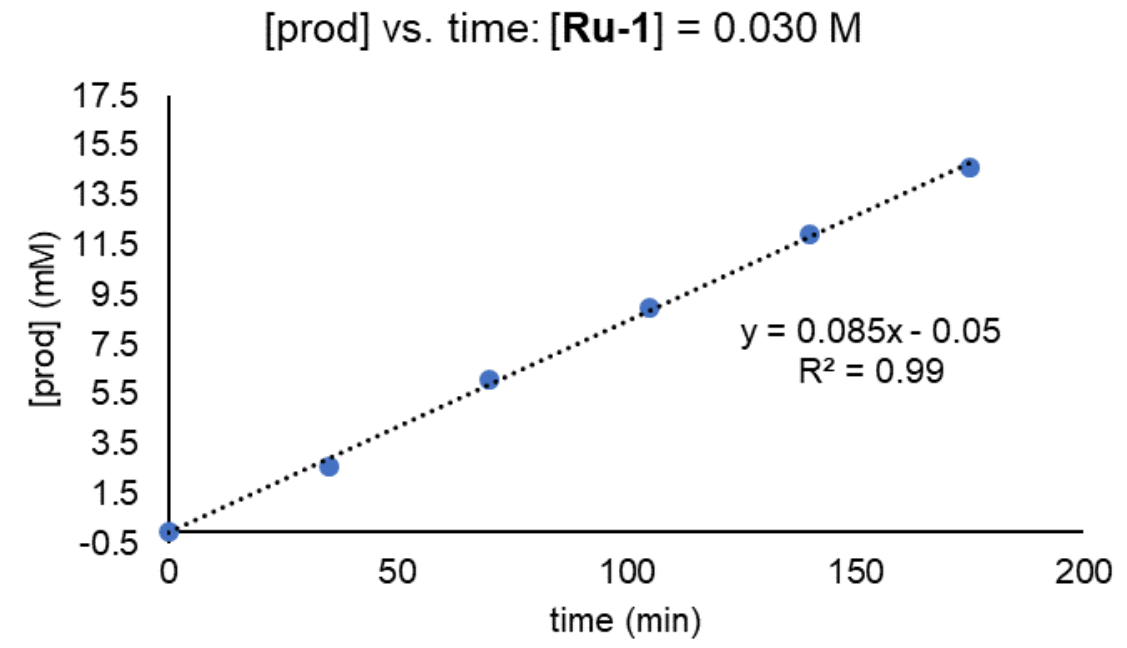

Figure S12. Rate of product formation as a function of time when $[\mathbf{R u - 1}]=0.030 \mathrm{M}$.

[prod] vs. time: $[\mathbf{R u}-1]=0.040 \mathrm{M}$

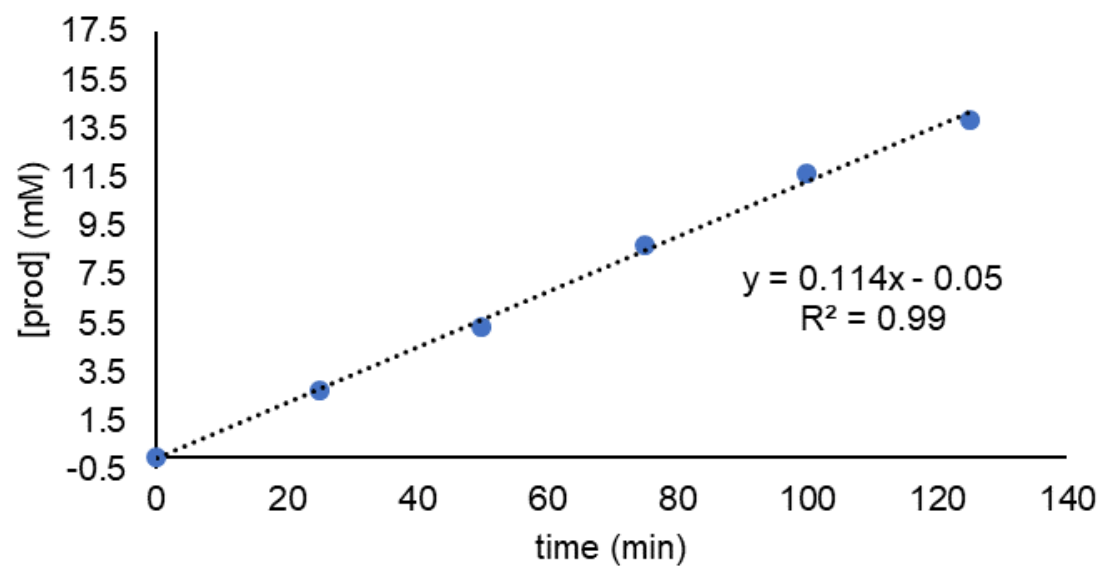

Figure S13. Rate of product formation as a function of time when [Ru-1] $=0.040 \mathrm{M}$.

\section{Order in PEt3 (Figure S14-S17)}

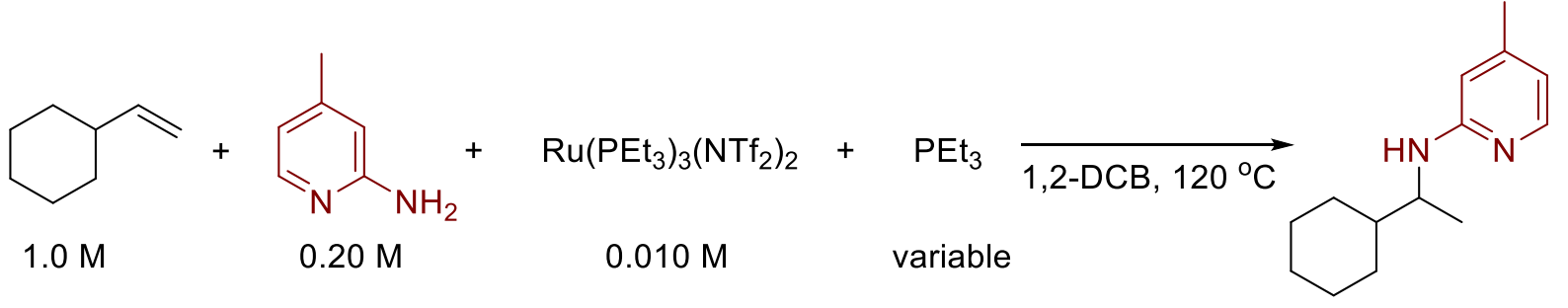




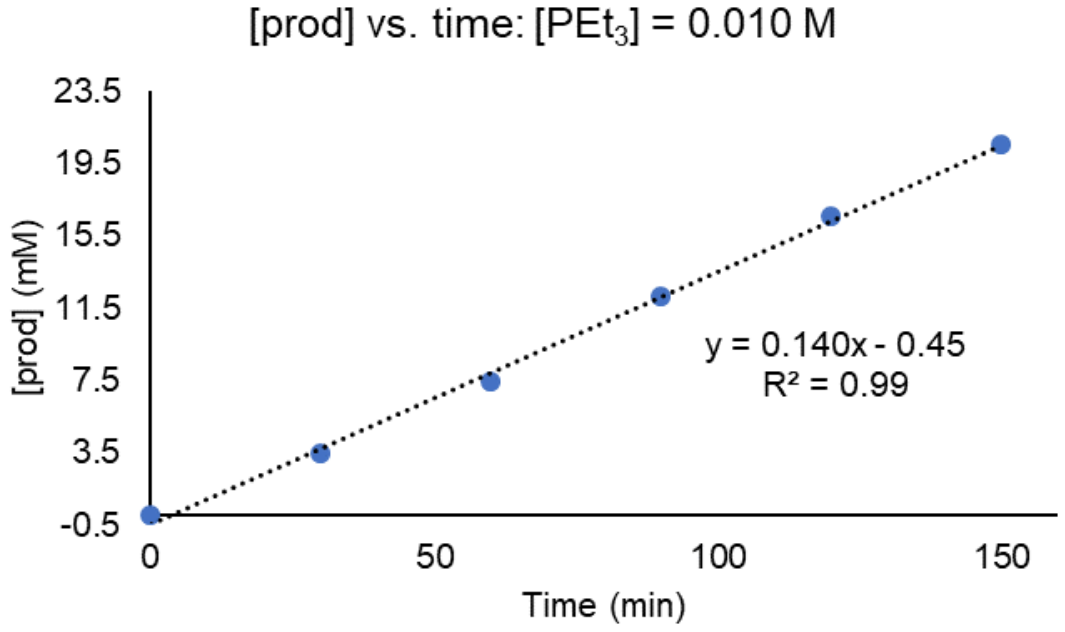

Figure S14. Rate of product formation as a function of time when $\left[\mathrm{PEt}_{3}\right]=0.010 \mathrm{M}$.

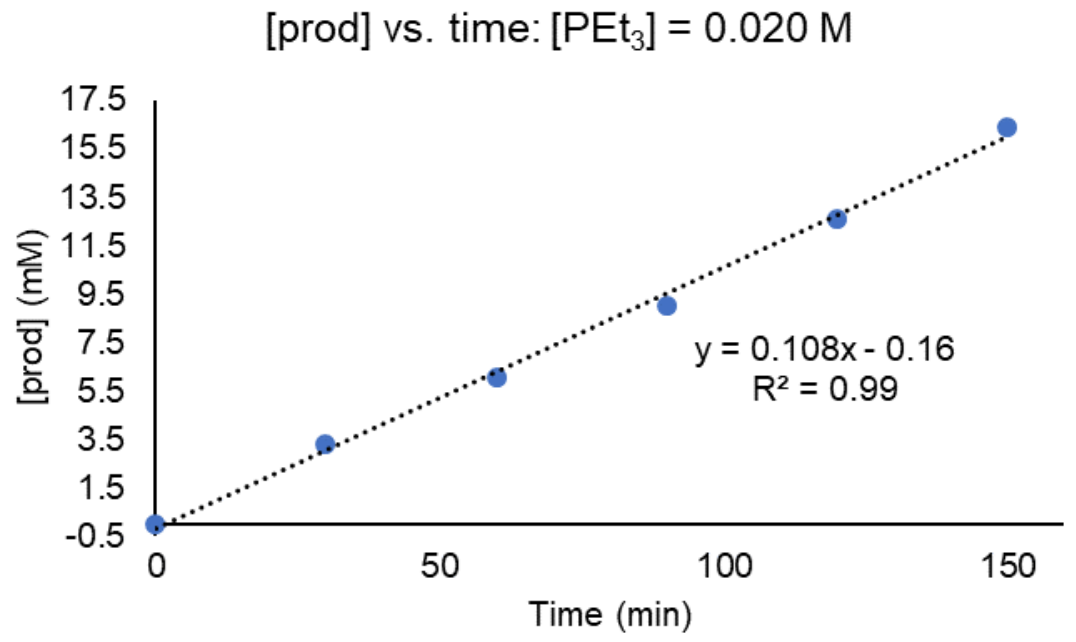

Figure S15. Rate of product formation as a function of time when $\left[\mathrm{PEt}_{3}\right]=0.020 \mathrm{M}$. 
[prod] vs. time: $\left[\mathrm{PEt}_{3}\right]=0.030 \mathrm{M}$

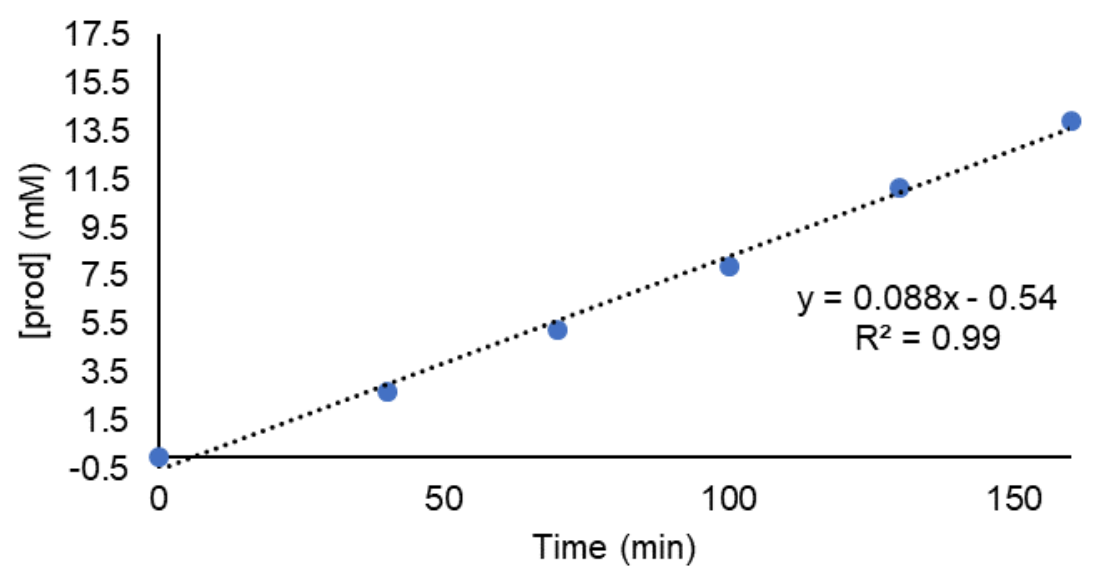

Figure S16. Rate of product formation as a function of time when $\left[\mathrm{PEt}_{3}\right]=0.030 \mathrm{M}$.

[prod] vs. time: $\left[\mathrm{PEt}_{3}\right]=0.040 \mathrm{M}$

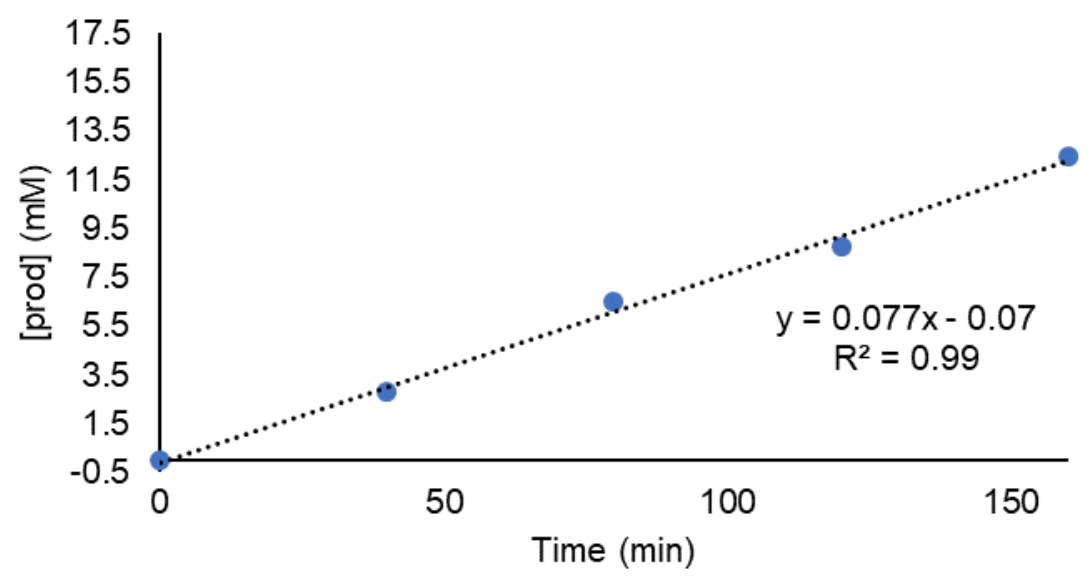

Figure S17. Rate of product formation as a function of time when $\left[\mathrm{PEt}_{3}\right]=0.040 \mathrm{M}$.

\section{Studies on the Origin of PEt 3 Inhibition}

\section{Low Temperature NMR Spectroscopy}

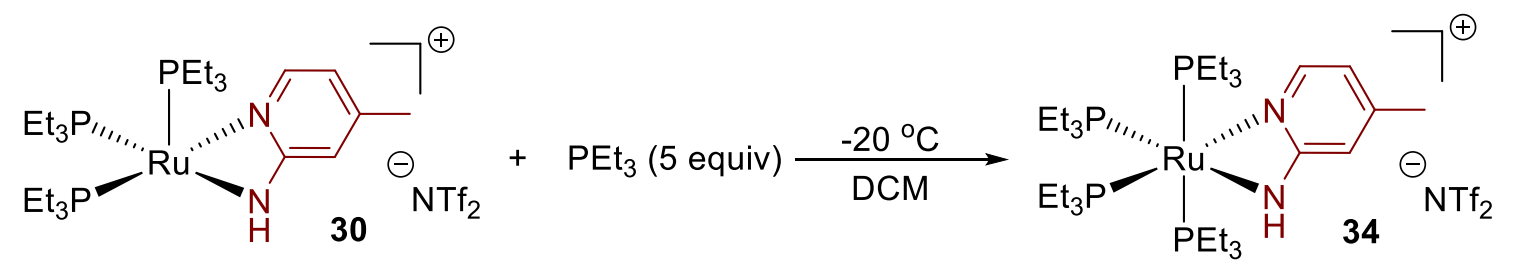

$\mathrm{Ru}\left(\mathrm{PEt}_{3}\right)_{3}$ (amido)(NTf $)\left(\right.$ (30) $(8.4 \mathrm{mg}, 0.010 \mathrm{mmol}), \mathrm{PEt}_{3}(5.9 \mathrm{mg}, 7.4 \mu \mathrm{L}, 0.050 \mathrm{mmol})$ were combined with $\mathrm{CD}_{2} \mathrm{Cl}_{2}(0.50 \mathrm{~mL})$ in a J-young tube and ${ }^{31} \mathrm{P}$ NMR spectrum of this mixture was acquired at $-20{ }^{\circ} \mathrm{C}$ (Figure S18). 


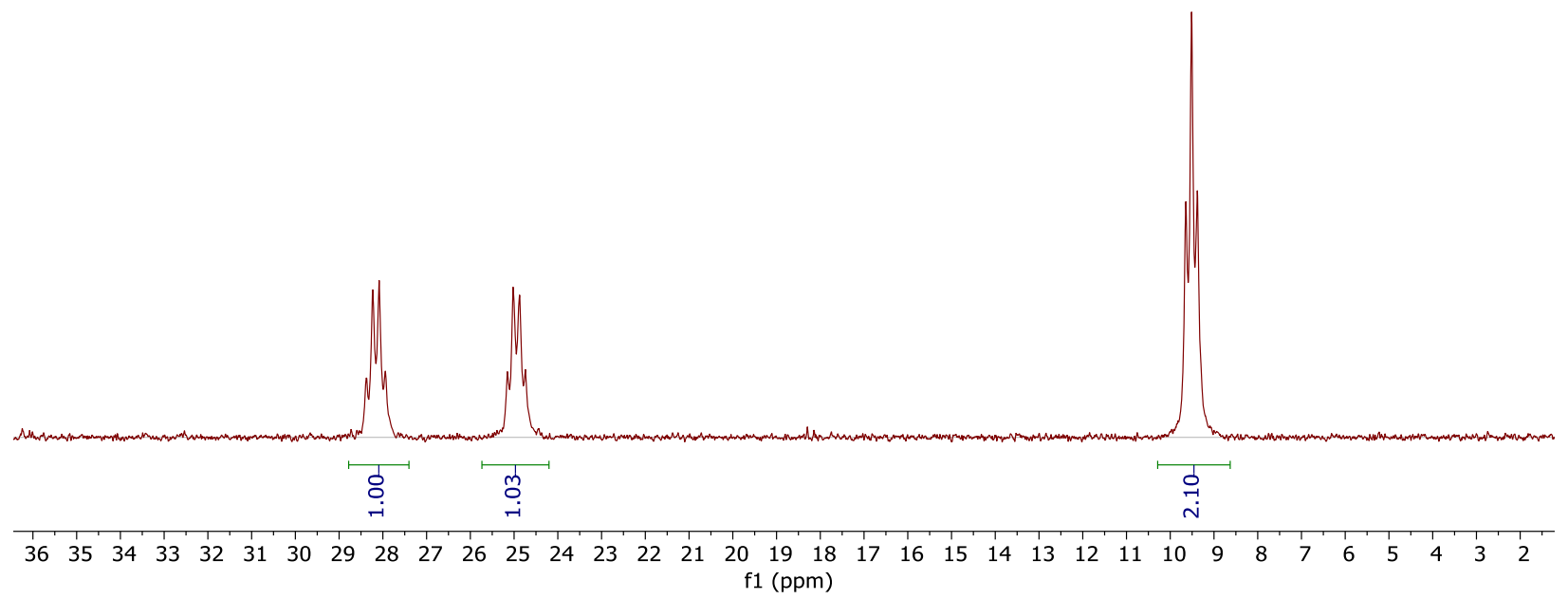

Figure S18. Low T NMR spectrum of Ru-amido complex with excess $\mathrm{PEt}_{3}$ (the $\mathrm{PEt}_{3}$ peak is not shown for clarity).

\section{Deuterium Labelling Experiment}

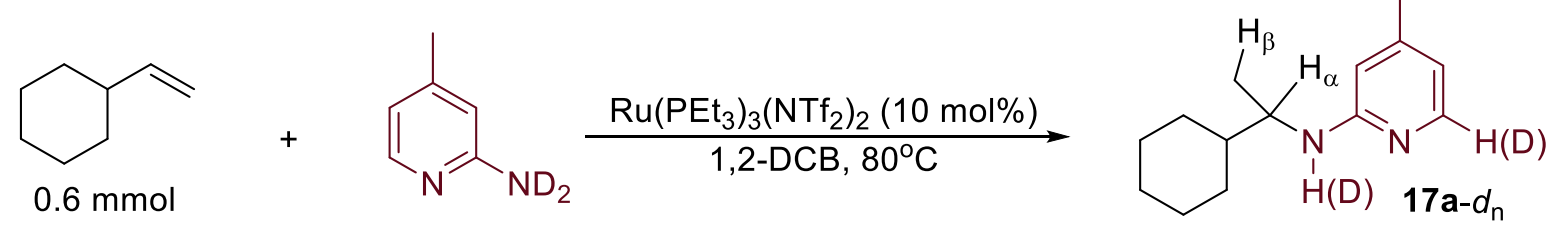

In a nitrogen-filled glovebox, vinylcyclohexane $(66 \mu \mathrm{L}, 53 \mathrm{mg}, 0.48 \mathrm{mmol})$, 2-amino-4methylpyridine- $\mathrm{D}_{2}$ (66 mg, $0.60 \mathrm{mmol}, 80 \%$ deuteration), $\mathrm{Ru}\left(\mathrm{PEt}_{3}\right)_{3}\left(\mathrm{NTf}_{2}\right)_{2}(49 \mathrm{mg}, 0.050 \mathrm{mmol}$, $10 \mathrm{~mol} \%), 1,3,5$-trimethoxybenzene $(11 \mathrm{mg}, 0.060 \mathrm{mmol})$ and DCB-D 4 (11 $\mathrm{mg}, 0.070 \mathrm{mmol})$ were dissolved in DCB $(0.32 \mathrm{~mL})$ in a $4 \mathrm{~mL}$ vial. After all the solid material was dissolved, the mixture was evenly distributed into 4 vials equipped with a stir bar. The mixture in each vial was heated at $80{ }^{\circ} \mathrm{C}$ for $0,24,36$ and 48 hours respectively and analyzed by ${ }^{1} \mathrm{H}$ and ${ }^{2} \mathrm{H}$ NMR spectroscopy (Table S1).

\begin{tabular}{|c|c|c|c|}
\hline Reaction Time & $\%$ Yield & $\% \mathrm{D}$ at $\mathrm{H}_{\alpha}$ position & $\% \mathrm{D}$ at $\mathrm{H}_{\beta}$ position \\
\hline $24 \mathrm{~h}$ & $13 \%$ & $29 \%$ & $29 \%$ \\
\hline $36 \mathrm{~h}$ & $18 \%$ & $31 \%$ & $32 \%$ \\
\hline $48 \mathrm{~h}$ & $25 \%$ & $29 \%$ & $28 \%$ \\
\hline
\end{tabular}

Table S1. \% Yield and \% D incorporation at $\mathrm{H}_{\alpha}$ and $\mathrm{H}_{\beta}$ position at 24, 36 and $48 \mathrm{~h}$ of the reaction between vinylcyclohexane and 2-amino-4-methylpyridine- $\mathrm{D}_{2}$.

\section{Catalytic Hydroamination with Acetone as an Additive}




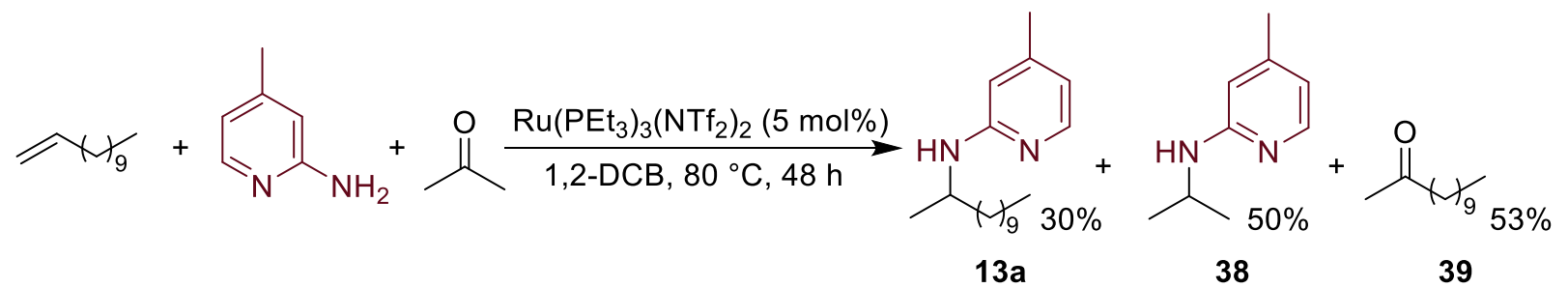

1-Dodecene ( $44 \mu \mathrm{L}, 34 \mathrm{mg}, 0.20 \mathrm{mmol}$ ), 2-amino-4-methylpyridine ( $22 \mathrm{mg}, 0.20 \mathrm{mmol}$ ), acetone $(15 \mu \mathrm{L}, 0.20 \mathrm{mmol})$ and $\mathrm{Ru}\left(\mathrm{PEt}_{3}\right)_{3}\left(\mathrm{NTf}_{2}\right)_{2}(10 \mathrm{mg}, 0.010 \mathrm{mmol}, 5.0 \mathrm{~mol} \%)$ were combined with DCB $(60 \mu \mathrm{L})$ and a magnetic stir bar in a one dram vial. The vial was capped, and the resulting mixture was heated at $80{ }^{\circ} \mathrm{C}$ with stirring for 48 hours. The mixture was allowed to cool and analyzed by ${ }^{1} \mathrm{H}$ NMR spectroscopy using 1,3,5-trimethoxybenzene as the internal standard and gas chromatography.

\section{Synthesis, Characterization, and Reactivity of the Hydridoruthenium Intermediate}

\section{Catalytic Imine Hydrogenation with $\operatorname{Ru}\left(\operatorname{PEt}_{3}\right)_{3}\left(\mathbf{N T f}_{2}\right)_{2}$}

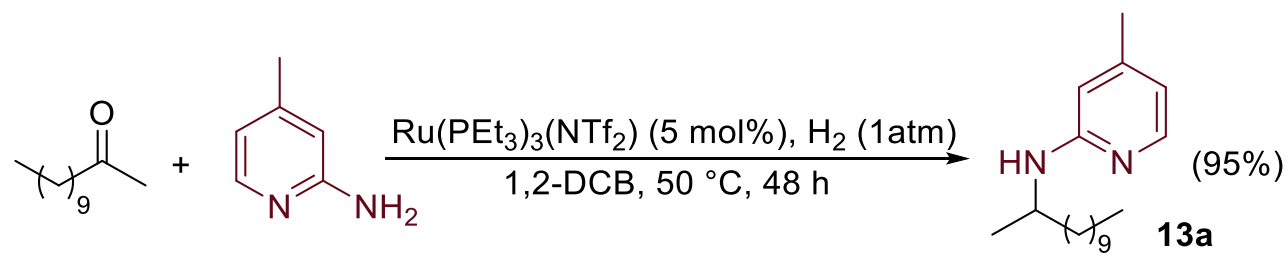

2-dodecanone (37 mg, $0.20 \mathrm{mmol}), \quad$ 2-amino-4-methylpyridine (22 $\mathrm{mg}, 0.20 \mathrm{mmol}$ ), $\mathrm{Ru}\left(\mathrm{PEt}_{3}\right)_{3}$ (amido)(NTf $)(8.4 \mathrm{mg}, 0.010 \mathrm{mmol}, 5.0 \mathrm{~mol} \%), 1,2-\mathrm{DCB}(0.20 \mathrm{~mL})$ and a magnetic stir bar were combined in a sealed tube equipped with a $\mathrm{H}_{2}$ balloon. The flask was heated at 50 ${ }^{\circ} \mathrm{C}$ with stirring for 48 hours. The mixture was allowed to cool and analyzed by ${ }^{1} \mathrm{H} \mathrm{NMR}$ spectroscopy using 1,3,5-trimethoxybenzene as the internal standard.

\section{Synthesis of the Potential Intermediate for Imine Hydrogenation}

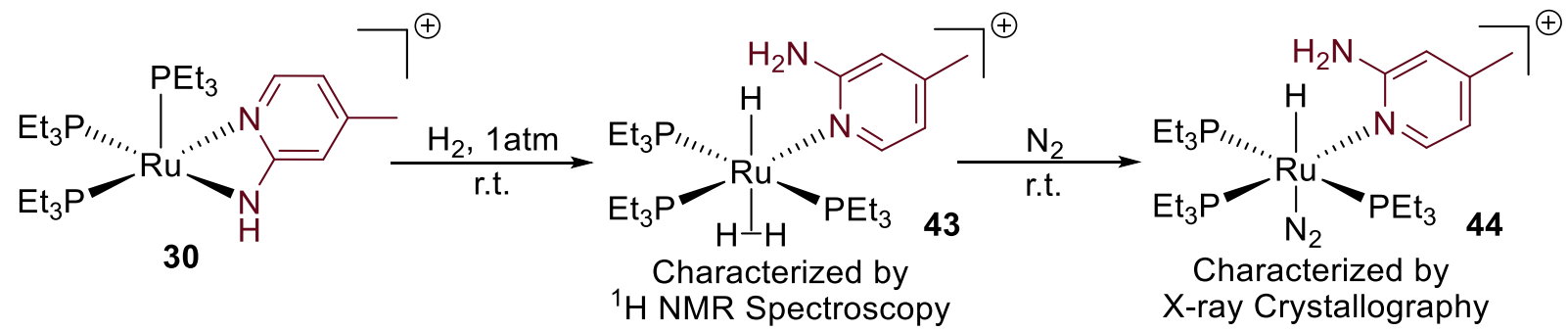

$\mathrm{Ru}\left(\mathrm{PEt}_{3}\right)_{3}$ (amido)(NTf $)_{2}(\mathbf{3 0})(17 \mathrm{mg}, 0.020 \mathrm{mmol})$ was dissolved in DCB-D $4(0.50 \mathrm{~mL})$ in a Jyoung tube. The tube was then sealed and connected to a Schlenk manifold and the $\mathrm{N}_{2}$ atmosphere in the tube was removed by freeze-pump-thaw. $\mathrm{H}_{2}(1.0 \mathrm{~atm})$ was added to the tube and the mixture was allowed to warm to room temperature. The mixture turned yellow, and was analyzed by NMR spectroscopy (88\% NMR yield before crystallization). ${ }^{1} \mathrm{H}$ NMR (400 MHz, $\left.\mathrm{CD}_{2} \mathrm{Cl}_{2}\right) \delta 7.67(\mathrm{~d}, J=5.5 \mathrm{~Hz}, 1 \mathrm{H}), 6.38(\mathrm{~s}, 1 \mathrm{H}), 6.20(\mathrm{~d}, J=5.5 \mathrm{~Hz}, 1 \mathrm{H}), 5.91-6.68(\mathrm{~s}, 2 \mathrm{H}), 2.19$ $(\mathrm{s}, 3 \mathrm{H}), 1.55-1.21(\mathrm{~m}, 18 \mathrm{H}), 1.02(\mathrm{dp}, J=22.2,7.7 \mathrm{~Hz}, 27 \mathrm{H}),-2.98(\mathrm{~s}, 2 \mathrm{H}),-9.22(\mathrm{q}, J=21.7$ 
$\mathrm{Hz}, 1 \mathrm{H}) .{ }^{31} \mathrm{P}$ NMR (162 MHz, $\left.\mathrm{CD}_{2} \mathrm{Cl}_{2}\right) \delta 39.45,28.16$. Single crystals suitable for x-ray crystallography analysis was obtained by layering ${ }^{i} \operatorname{Pr}_{2} \mathrm{O}$ and pentane onto the mixture. Due to the instability of this hydridoruthenium aminopyridyl complex 44, we were unable to obtain high quality NMR spectroscopic data.

\section{DFT Computational Studies}

Initial conformational search and preoptimization of all reported structures were conducted using the (Geometry, Frequency, Noncovalent, eXtended Tight Binding") GFN-xTB method to account for the conformational flexibility of the triethylphosphine ligands. ${ }^{3}$ The GFN2-xTB implementation of DFT tight binding was used and CREST was used for conformational search. ${ }^{4-6}$ The ten GFN2-xTB optimized structures that was found by CREST with the lowest energies were then used as inputs for subsequent DFT optimization.

All DFT calculations were performed with the Gaussian 16 software package. Geometry optimization for all reported structures were performed in the gas-phase using the B3LYP-D3BJ /[6-31G+(d) + Lanl2dz (for Ru)] level of theory with the corresponding Hay-Wadt effective core potential for $\mathrm{Ru}$ and Grimme's empirical dispersion correction with Becke-Johnson damping for B3LYP. ${ }^{7}$ Frequency calculations were performed on all reported structures to ensure that each minimum-energy structure has no imaginary frequencies and each transition state (TS) structure has precisely one imaginary frequency. Thermal corrections for Gibbs free energy (G) and enthalpy $(\mathrm{H})$ were calculated at the same level of theory and the free energies were corrected at $353.15 \mathrm{~K} .{ }^{8}$ The electronic energy of every optimized structure was further recalculated under the B3LYP-D3BJ/[6-311+G(d,p) + SDD (for Ru)] level of theory. Bulk solvent effects were taken into consideration for single point energy calculations using the self-consistent reaction field SMD model (IEF-SMD) with chlorobenzene as the solvent. ${ }^{9}$ The thermal corrections were applied to the recalculated electronic energies to give the final Gibbs free energy and enthalpy values.

Energy Data for all Reported Structures

\begin{tabular}{|c|c|c|c|c|c|}
\hline Structures & E (Hartree) & ZPE (Hartree) & H (Hartree) & qh-G (Hartree) & $\begin{array}{c}\text { Imaginary } \\
\text { Frequency }\left(\mathrm{cm}^{-1}\right)\end{array}$ \\
\hline Propene & -117.954319 & -117.839391 & -117.834906 & -117.9012 & - \\
\hline 1b & -343.108724 & -342.876285 & -342.867932 & -343.01048 & - \\
\hline Enamine & -459.863233 & -459.541634 & -459.529619 & -459.70979 & - \\
\hline Imine & -459.866639 & -459.551747 & -459.539461 & -459.71122 & - \\
\hline Product & -461.093957 & -460.749437 & -460.736895 & -460.91768 & - \\
\hline $\mathbf{3 0}$ & -2175.003901 & -2172.772013 & -2172.728276 & -2174.3525 & - \\
\hline $\mathbf{3 2}$ & -2292.961218 & -2290.616426 & -2290.569029 & -2292.2278 & - \\
\hline TS1 & -2292.941595 & -2290.601895 & -2290.555350 & -2292.2079 & -317.14 \\
\hline $\mathbf{3 3}$ & -2292.97462 & -2290.634056 & -2290.586816 & -2292.2408 & - \\
\hline TS2 & -2292.954251 & -2290.611982 & -2290.565490 & -2292.2229 & -675.89 \\
\hline $\mathbf{4 5}$ & -2292.971315 & -2290.629788 & -2290.581564 & -2292.2443 & - \\
\hline $\mathbf{4 6}$ & -2176.216361 & -2173.964766 & -2173.920112 & -2175.5462 & - \\
\hline
\end{tabular}


Table S2. Electronic energies (E), zero-point energy corrections (ZPE), enthalpies (H), quasiharmonic Gibbs free energies calculated at $\mathrm{T}=353.15 \mathrm{~K}$ (qh-G), and imaginary frequencies of all reported structures.

\section{Cartesian Coordinates (̊) of Optimized Structures}

$\begin{array}{lrrr}\text { Propene } & & & \\ & & & \\ \mathrm{C} & -1.28406500 & -0.22563000 & -0.00001400 \\ \mathrm{H} & -1.29932000 & -1.31508800 & -0.00005000 \\ \mathrm{H} & -2.24911000 & 0.27458000 & -0.00000100 \\ \mathrm{C} & -0.13787900 & 0.46174400 & 0.00001200 \\ \mathrm{H} & -0.18438100 & 1.55116000 & 0.00004700 \\ \mathrm{C} & 1.24131500 & -0.15856800 & 0.00000200 \\ \mathrm{H} & 1.39546000 & -0.79065500 & -0.88371300 \\ \mathrm{H} & 2.02560000 & 0.60527300 & -0.00007300 \\ \mathrm{H} & 1.39552300 & -0.79054300 & 0.88378700\end{array}$

2-amino-4-methylpyridine (1b)

$\begin{array}{lrrr} & & & \\ \mathrm{C} & -0.17223800 & 1.73587100 & 0.00297100 \\ \mathrm{C} & 1.09708300 & 1.17006600 & 0.00476600 \\ \mathrm{C} & 1.20812700 & -0.23240400 & 0.00135600 \\ \mathrm{C} & 0.03309600 & -0.97481300 & -0.00708600 \\ \mathrm{C} & -1.20890700 & -0.30551500 & -0.00701700 \\ \mathrm{~N} & -1.31276700 & 1.03013600 & 0.00176900 \\ \mathrm{H} & -0.29218300 & 2.81770900 & 0.00712400 \\ \mathrm{H} & 1.98143600 & 1.80094200 & 0.01027000 \\ \mathrm{H} & 0.06601300 & -2.06170800 & -0.02373000 \\ \mathrm{C} & 2.55914800 & -0.90161200 & 0.00244200 \\ \mathrm{H} & 3.14255100 & -0.60542100 & -0.87791900 \\ \mathrm{H} & 3.13889600 & -0.61176000 & 0.88735800 \\ \mathrm{H} & 2.46819300 & -1.99236300 & -0.00197500 \\ \mathrm{~N} & -2.39959600 & -1.01293700 & -0.06880600 \\ \mathrm{H} & -2.39936500 & -1.95095600 & 0.30905100 \\ \mathrm{H} & -3.21685800 & -0.46639700 & 0.17449100\end{array}$

4-methyl-N-(prop-1-en-2-yl)pyridin-2-amine (Enamine)<smiles>C=C(C)Nc1cc(C)ccn1</smiles> 


$\begin{array}{lrrr}\mathrm{C} & -2.47834100 & 1.00608800 & 0.00021900 \\ \mathrm{C} & -2.36369200 & -0.39833300 & -0.00004200 \\ \mathrm{C} & -1.08688600 & -0.93843700 & -0.00015200 \\ \mathrm{C} & 0.03903700 & -0.07882700 & 0.00003500 \\ \mathrm{~N} & -0.07482100 & 1.25303200 & 0.00023400 \\ \mathrm{H} & -1.37078300 & 2.85180200 & 0.00049800 \\ \mathrm{H} & -3.45221200 & 1.48742200 & 0.00032900 \\ \mathrm{H} & -0.94829400 & -2.01788400 & -0.00037900 \\ \mathrm{C} & -3.58991100 & -1.27504800 & -0.00021900 \\ \mathrm{H} & -4.21077900 & -1.07872500 & 0.88247000 \\ \mathrm{H} & -4.21078700 & -1.07835500 & -0.88281900 \\ \mathrm{H} & -3.32600300 & -2.33732800 & -0.00044100 \\ \mathrm{~N} & 1.29728200 & -0.65695000 & -0.00001000 \\ \mathrm{H} & 1.28553800 & -1.66711800 & -0.00023400 \\ \mathrm{C} & 3.65415200 & -1.14870100 & 0.00048500 \\ \mathrm{H} & 3.57812200 & -1.79252000 & 0.88800200 \\ \mathrm{H} & 3.57739500 & -1.79446100 & -0.88556300 \\ \mathrm{H} & 4.64614600 & -0.69188700 & -0.00045300 \\ \mathrm{C} & 2.58059300 & -0.08624500 & -0.00018500 \\ \mathrm{C} & 2.87098200 & 1.22688700 & -0.00068100 \\ \mathrm{H} & 3.91408700 & 1.52592400 & -0.00070000 \\ \mathrm{H} & 2.10682700 & 1.98890700 & -0.00097200\end{array}$

N-(4-methylpyridin-2-yl)propan-2-imine (Imine)

$\begin{array}{lrrr} & & \\ \mathrm{C} & 1.30737900 & 1.72942400 & -0.35818100 \\ \mathrm{C} & 2.43384200 & 0.96695000 & -0.06674200 \\ \mathrm{C} & 2.29078100 & -0.42129900 & 0.09666500 \\ \mathrm{C} & 1.01288100 & -0.95302300 & -0.05259100 \\ \mathrm{C} & -0.07222300 & -0.09889500 & -0.32239300 \\ \mathrm{~N} & 0.06926500 & 1.22692900 & -0.47834600 \\ \mathrm{H} & 1.39457600 & 2.80504200 & -0.50226900 \\ \mathrm{H} & 3.40777800 & 1.44135100 & 0.02130600 \\ \mathrm{H} & 0.83258200 & -2.02055900 & 0.03121100 \\ \mathrm{C} & 3.47926900 & -1.29552200 & 0.40695100 \\ \mathrm{H} & 4.25717500 & -1.18688700 & -0.35849700 \\ \mathrm{H} & 3.92940400 & -1.01784700 & 1.36815500 \\ \mathrm{H} & 3.19671200 & -2.35149100 & 0.45757600 \\ \mathrm{~N} & -1.33190100 & -0.67931200 & -0.52111800 \\ \mathrm{C} & -2.38935000 & -0.23799400 & 0.05165900 \\ \mathrm{C} & -2.45443800 & 0.86636500 & 1.08272200 \\ \mathrm{H} & -2.71942100 & 1.81184000 & 0.59377000 \\ \mathrm{H} & -3.23148900 & 0.64401900 & 1.82310300 \\ \mathrm{H} & -1.49839200 & 1.02015900 & 1.58632400 \\ \mathrm{C} & -3.70625600 & -0.87301100 & -0.31167900\end{array}$




$\begin{array}{llll}\mathrm{H} & -4.15272500 & -1.35941500 & 0.56611700 \\ \mathrm{H} & -4.41859900 & -0.10609900 & -0.64435400 \\ \mathrm{H} & -3.57046300 & -1.61140600 & -1.10465500\end{array}$

N-isopropyl-4-methylpyridin-2-amine (Product)

$$
\begin{array}{lrrr} 
& & & \\
\mathrm{C} & & & \\
\mathrm{N} & -1.33402500 & 1.73458400 & -0.33785000 \\
\mathrm{C} & -0.10699300 & 1.18980700 & -0.34158200 \\
\mathrm{C} & -0.02051100 & -0.13596000 & -0.15371300 \\
\mathrm{C} & -1.16241100 & -0.94645300 & 0.05939100 \\
\mathrm{C} & -2.42310000 & -0.36896500 & 0.06477400 \\
\mathrm{H} & -2.50921900 & 1.02225700 & -0.14348200 \\
\mathrm{C} & -3.47003100 & 1.52914900 & -0.14836500 \\
\mathrm{H} & -3.66508200 & -1.19486700 & 0.28513400 \\
\mathrm{H} & -4.34070200 & -1.12157400 & -0.57600000 \\
\mathrm{H} & -4.22013300 & -0.84082400 & 1.16257500 \\
\mathrm{H} & -3.42427900 & -2.25159000 & 0.43862500 \\
\mathrm{~N} & -1.04569600 & -2.01795300 & 0.20846400 \\
\mathrm{H} & 1.23087000 & -0.71107000 & -0.20851600 \\
\mathrm{C} & 1.29215000 & -1.65358500 & 0.15618800 \\
\mathrm{C} & 2.46694200 & 0.07156600 & -0.14800300 \\
\mathrm{H} & 3.62131000 & -0.79669400 & -0.65042400 \\
\mathrm{H} & 3.43224100 & -1.14890200 & -1.67011600 \\
\mathrm{H} & 4.55785600 & -0.22862000 & -0.64535400 \\
\mathrm{C} & 3.76503800 & -1.67395500 & -0.00382500 \\
\mathrm{H} & 2.73107900 & 0.62250700 & 1.26008400 \\
\mathrm{H} & 2.87374300 & -0.19747100 & 1.97648700 \\
\mathrm{H} & 1.88853300 & 1.23588200 & 1.59269900 \\
\mathrm{H} & 3.63476700 & 1.24436700 & 1.27044800 \\
\mathrm{H} & 2.32448800 & 0.91477800 & -0.82956400 \\
& -1.36500900 & 2.81128900 & -0.49703200
\end{array}
$$

\section{Ru-amido Complex (30)}

$$
\begin{array}{llll} 
& & \\
\mathrm{Et}_{3} \mathrm{P}_{2 \ldots \ldots}
\end{array}
$$




\begin{tabular}{|c|c|c|c|}
\hline $\mathrm{N}$ & -1.19644900 & 1.81014100 & 0.84771500 \\
\hline $\mathrm{C}$ & -3.40275800 & -0.80707600 & 0.24402200 \\
\hline $\mathrm{H}$ & -3.25958800 & -1.82580600 & -0.10167300 \\
\hline $\mathrm{C}$ & -4.66839200 & -0.34715600 & 0.56838200 \\
\hline $\mathrm{H}$ & -5.52607900 & -1.00511300 & 0.47486200 \\
\hline $\mathrm{C}$ & -4.82074300 & 0.98579600 & 1.01532000 \\
\hline $\mathrm{C}$ & -6.18543300 & 1.51572100 & 1.36788700 \\
\hline $\mathrm{H}$ & -6.14284100 & 2.55854800 & 1.69446900 \\
\hline $\mathrm{H}$ & -6.63535500 & 0.92221500 & 2.17290200 \\
\hline $\mathrm{H}$ & -6.86085300 & 1.45308800 & 0.50617800 \\
\hline $\mathrm{C}$ & -3.68656100 & 1.78263100 & 1.12576900 \\
\hline $\mathrm{H}$ & -3.75561500 & 2.80651500 & 1.48236400 \\
\hline $\mathrm{C}$ & -2.42582100 & 1.24841800 & 0.78415900 \\
\hline $\mathrm{C}$ & 1.85185800 & 2.95274800 & 0.81181500 \\
\hline $\mathrm{H}$ & 1.37312100 & 3.27062500 & -0.11753700 \\
\hline $\mathrm{H}$ & 1.12405000 & 3.15638900 & 1.60473900 \\
\hline $\mathrm{C}$ & 3.12965100 & 3.76459900 & 1.04886000 \\
\hline $\mathrm{H}$ & 3.83682200 & 3.66077800 & 0.22013400 \\
\hline $\mathrm{H}$ & 3.64075300 & 3.47471900 & 1.97256300 \\
\hline $\mathrm{H}$ & 2.88075500 & 4.82822500 & 1.13390200 \\
\hline $\mathrm{C}$ & 3.49644800 & 0.75593000 & -0.28248800 \\
\hline $\mathrm{H}$ & 3.36091100 & -0.24614700 & -0.69719800 \\
\hline $\mathrm{H}$ & 3.45010500 & 1.44548400 & -1.13476000 \\
\hline $\mathrm{C}$ & 4.87038700 & 0.84545400 & 0.39631100 \\
\hline $\mathrm{H}$ & 5.65711900 & 0.62630800 & -0.33408300 \\
\hline $\mathrm{H}$ & 4.96765100 & 0.11902000 & 1.20848300 \\
\hline $\mathrm{H}$ & 5.06823500 & 1.83647000 & 0.80968400 \\
\hline $\mathrm{C}$ & 2.48158000 & 0.69129400 & 2.49267300 \\
\hline $\mathrm{H}$ & 2.82067300 & -0.34844900 & 2.50967800 \\
\hline $\mathrm{H}$ & 3.34343700 & 1.30625900 & 2.77322900 \\
\hline $\mathrm{C}$ & 1.33609000 & 0.88399200 & 3.49491900 \\
\hline $\mathrm{H}$ & 0.46736200 & 0.26785900 & 3.24188300 \\
\hline $\mathrm{H}$ & 0.99603100 & 1.92316900 & 3.53242000 \\
\hline $\mathrm{H}$ & 1.66340500 & 0.60520900 & 4.50214000 \\
\hline $\mathrm{C}$ & -1.57702100 & 0.28434100 & -2.83364900 \\
\hline $\mathrm{H}$ & -2.28935500 & 0.85432500 & -2.23045300 \\
\hline $\mathrm{H}$ & -1.48349900 & 0.79978500 & -3.79764600 \\
\hline $\mathrm{C}$ & -2.08652200 & -1.14206500 & -3.04341500 \\
\hline $\mathrm{H}$ & -3.06975100 & -1.12097300 & -3.52497200 \\
\hline $\mathrm{H}$ & -2.19214700 & -1.66588100 & -2.09412700 \\
\hline $\mathrm{H}$ & -1.41850800 & -1.72796100 & -3.68334500 \\
\hline $\mathrm{C}$ & 1.18345400 & -0.67504600 & -2.95880100 \\
\hline $\mathrm{H}$ & 2.18532800 & -0.55966800 & -2.53337900 \\
\hline $\mathrm{H}$ & 0.87098500 & -1.70015100 & -2.73657800 \\
\hline $\mathrm{C}$ & 1.22678700 & -0.46051400 & -4.47787300 \\
\hline $\mathrm{H}$ & 0.23218200 & -0.51685200 & -4.93073900 \\
\hline
\end{tabular}




$\begin{array}{lrrr}\mathrm{H} & 1.84419200 & -1.23693400 & -4.94285300 \\ \mathrm{H} & 1.66546400 & 0.50554700 & -4.74341700 \\ \mathrm{C} & 0.58785300 & 2.12797300 & -2.52011200 \\ \mathrm{H} & 1.56785700 & 2.33153500 & -2.07709000 \\ \mathrm{H} & 0.73954000 & 2.09036100 & -3.60438700 \\ \mathrm{C} & -0.41249600 & 3.23728600 & -2.17602200 \\ \mathrm{H} & -1.32876000 & 3.13993300 & -2.76651200 \\ \mathrm{H} & 0.01916500 & 4.21909300 & -2.39872200 \\ \mathrm{H} & -0.69621500 & 3.20554400 & -1.12150900 \\ \mathrm{C} & 0.46357700 & -2.60157700 & 2.11116900 \\ \mathrm{H} & 0.72642800 & -3.66162900 & 2.20326100 \\ \mathrm{H} & 1.21979600 & -2.04182600 & 2.67072200 \\ \mathrm{C} & -0.92834400 & -2.33698000 & 2.70077400 \\ \mathrm{H} & -1.70538600 & -2.90472400 & 2.17900000 \\ \mathrm{H} & -1.20563400 & -1.27971000 & 2.63500300 \\ \mathrm{H} & -0.95596400 & -2.62690000 & 3.75636100 \\ \mathrm{C} & -0.56504800 & -3.27557500 & -0.51754600 \\ \mathrm{H} & -1.57074300 & -2.87376500 & -0.37813200 \\ \mathrm{H} & -0.35702100 & -3.20289100 & -1.59132500 \\ \mathrm{C} & -0.52743500 & -4.74107300 & -0.06890900 \\ \mathrm{H} & -1.19845200 & -5.33883500 & -0.69560500 \\ \mathrm{H} & -0.85861400 & -4.85590300 & 0.96749700 \\ \mathrm{H} & 0.47169500 & -5.18095400 & -0.15240500 \\ \mathrm{C} & 2.24590900 & -2.79891200 & -0.19589900 \\ \mathrm{H} & 2.08068300 & -3.84668400 & -0.46823100 \\ \mathrm{H} & 2.53686700 & -2.28657400 & -1.11736100 \\ \mathrm{C} & 3.38012600 & -2.72495700 & 0.83144400 \\ \mathrm{H} & 4.30324500 & -3.12556100 & 0.39868000 \\ \mathrm{H} & 3.15448200 & -3.31224900 & 1.72627700 \\ \mathrm{H} & 3.58649600 & -1.70035800 & 1.14627200 \\ \mathrm{H} & -1.14145300 & 2.78574500 & 1.11397100\end{array}$

Ru-alkene Complex (32)

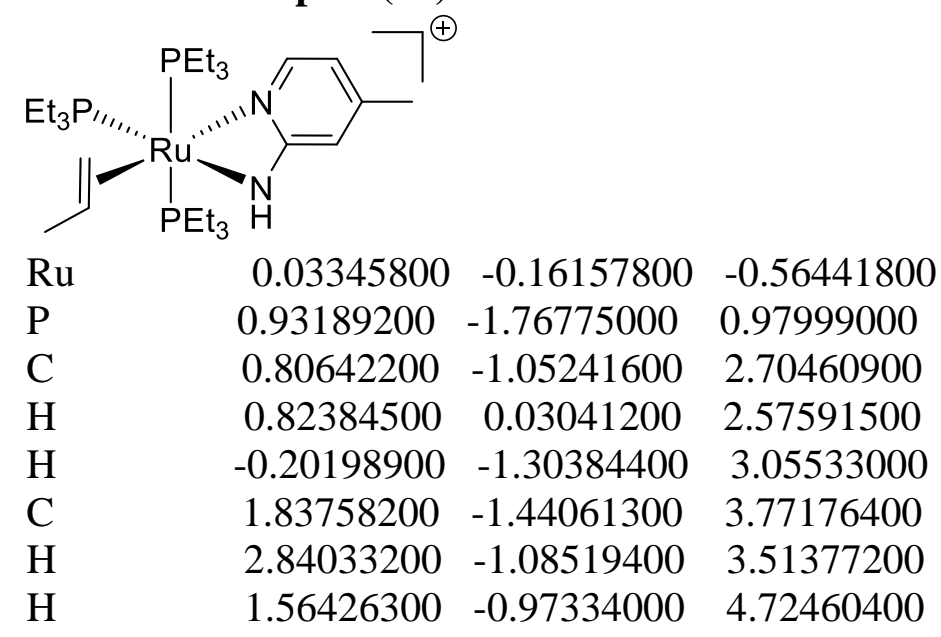




\begin{tabular}{|c|c|c|c|}
\hline $\mathrm{H}$ & 1.89510500 & -2.51904700 & 3.93814100 \\
\hline $\mathrm{C}$ & 2.72086500 & -2.30283700 & 0.89214000 \\
\hline $\mathrm{C}$ & 3.08841300 & -3.40476000 & -0.11041300 \\
\hline $\mathrm{H}$ & 4.14571600 & -3.66428600 & 0.01183600 \\
\hline $\mathrm{H}$ & 2.94408300 & -3.10608400 & -1.14926600 \\
\hline $\mathrm{H}$ & 2.50989700 & -4.31826800 & 0.05564100 \\
\hline $\mathrm{H}$ & 3.32876500 & -1.41333400 & 0.71201200 \\
\hline $\mathrm{H}$ & 2.98832600 & -2.65075200 & 1.89337100 \\
\hline $\mathrm{C}$ & 0.07681900 & -3.41260100 & 1.12765800 \\
\hline $\mathrm{H}$ & -0.98904500 & -3.20076200 & 1.23277700 \\
\hline $\mathrm{C}$ & 0.51892200 & -4.34463500 & 2.26244600 \\
\hline $\mathrm{H}$ & 1.59181000 & -4.55695900 & 2.23770600 \\
\hline $\mathrm{H}$ & 0.27927500 & -3.92272600 & 3.24280800 \\
\hline $\mathrm{H}$ & -0.00711900 & -5.30243400 & 2.18180700 \\
\hline $\mathrm{H}$ & 0.19593100 & -3.91114200 & 0.15911600 \\
\hline $\mathrm{P}$ & 2.16788100 & 1.05852300 & -0.50793600 \\
\hline $\mathrm{C}$ & 2.85823100 & 1.47733700 & 1.17506600 \\
\hline $\mathrm{H}$ & 2.88794500 & 0.55595700 & 1.76384100 \\
\hline $\mathrm{C}$ & 2.09630500 & 2.56778300 & 1.93711100 \\
\hline $\mathrm{H}$ & 2.16364800 & 3.53364900 & 1.42749600 \\
\hline $\mathrm{H}$ & 1.03883200 & 2.33275000 & 2.05520500 \\
\hline $\mathrm{H}$ & 2.53180400 & 2.69404700 & 2.93463800 \\
\hline $\mathrm{H}$ & 3.89823800 & 1.78956700 & 1.04881900 \\
\hline $\mathrm{C}$ & 2.06992900 & 2.73492800 & -1.29989300 \\
\hline $\mathrm{C}$ & 3.36654700 & 3.55074200 & -1.35020200 \\
\hline $\mathrm{H}$ & 4.13187200 & 3.06352600 & -1.96235900 \\
\hline $\mathrm{H}$ & 3.78855700 & 3.72167700 & -0.35468900 \\
\hline $\mathrm{H}$ & 3.17055800 & 4.53273900 & -1.79528800 \\
\hline $\mathrm{H}$ & 1.68512100 & 2.57466800 & -2.31158800 \\
\hline $\mathrm{H}$ & 1.28815900 & 3.28362600 & -0.76636000 \\
\hline $\mathrm{C}$ & 3.63582500 & 0.28098600 & -1.38988300 \\
\hline $\mathrm{C}$ & 5.03623600 & 0.44380000 & -0.78508100 \\
\hline $\mathrm{H}$ & 5.33047100 & 1.49204200 & -0.68675100 \\
\hline $\mathrm{H}$ & 5.11341200 & -0.02068000 & 0.20348400 \\
\hline $\mathrm{H}$ & 5.77098100 & -0.04585700 & -1.43413600 \\
\hline $\mathrm{H}$ & 3.42403400 & -0.78398000 & -1.50376700 \\
\hline $\mathrm{H}$ & 3.62981000 & 0.69626400 & -2.40392800 \\
\hline $\mathrm{H}$ & 0.30984700 & 1.18484700 & -3.72579500 \\
\hline $\mathrm{C}$ & 0.79240500 & 0.20400600 & -3.68911200 \\
\hline $\mathrm{H}$ & 0.74440600 & -0.21331200 & -4.70466700 \\
\hline $\mathrm{H}$ & 1.84606900 & 0.34180900 & -3.44213900 \\
\hline $\mathrm{C}$ & 0.10289500 & -0.74398100 & -2.74602800 \\
\hline $\mathrm{C}$ & 0.76610000 & -1.73052400 & -2.02403300 \\
\hline $\mathrm{H}$ & 0.27610800 & -2.67133500 & -1.80016100 \\
\hline $\mathrm{H}$ & 1.84480500 & -1.77723500 & -2.08220900 \\
\hline $\mathrm{H}$ & -0.93848700 & -0.91231400 & -2.99641600 \\
\hline
\end{tabular}




$\begin{array}{lrrr}\mathrm{P} & -2.20956300 & -1.06475400 & -0.51818800 \\ \mathrm{C} & -3.50243900 & 0.02594800 & -1.28522100 \\ \mathrm{C} & -3.40586100 & 0.17850700 & -2.80617000 \\ \mathrm{H} & -3.47232500 & -0.78430900 & -3.32482600 \\ \mathrm{H} & -4.23260400 & 0.79947700 & -3.16845600 \\ \mathrm{H} & -2.47172100 & 0.66350500 & -3.09518000 \\ \mathrm{H} & -4.48199700 & -0.38038000 & -1.01711300 \\ \mathrm{H} & -3.42417500 & 1.00543000 & -0.80707000 \\ \mathrm{C} & -2.85809800 & -1.36855300 & 1.21672000 \\ \mathrm{H} & -2.03233500 & -1.14307500 & 1.89403400 \\ \mathrm{C} & -4.09547100 & -0.57790200 & 1.65888800 \\ \mathrm{H} & -3.94397500 & 0.50076500 & 1.56206900 \\ \mathrm{H} & -4.98582500 & -0.85362700 & 1.08665900 \\ \mathrm{H} & -4.30531600 & -0.78912500 & 2.71356000 \\ \mathrm{H} & -3.05791100 & -2.44199800 & 1.31269300 \\ \mathrm{C} & -2.51903900 & -2.69652600 & -1.35932900 \\ \mathrm{H} & -1.89288300 & -3.43620400 & -0.85041600 \\ \mathrm{C} & -3.97759900 & -3.17096300 & -1.38448400 \\ \mathrm{H} & -4.03999000 & -4.17218800 & -1.82480800 \\ \mathrm{H} & -4.60993800 & -2.50912700 & -1.98332700 \\ \mathrm{H} & -4.40824400 & -3.22734000 & -0.37884500 \\ \mathrm{H} & -2.13241400 & -2.62632100 & -2.38210000 \\ \mathrm{~N} & -0.81091400 & 1.64388600 & -1.33611100 \\ \mathrm{C} & -1.18931700 & 2.18079200 & -0.18090000 \\ \mathrm{~N} & -0.89783500 & 1.29122200 & 0.82957600 \\ \mathrm{C} & -1.31052900 & 1.56712400 & 2.08141800 \\ \mathrm{C} & -1.96149900 & 2.73931100 & 2.41214700 \\ \mathrm{C} & -2.20860600 & 3.70651300 & 1.40269200 \\ \mathrm{C} & -1.81777500 & 3.42140800 & 0.10541800 \\ \mathrm{H} & -2.00414000 & 4.12127700 & -0.70470800 \\ \mathrm{C} & -2.88990900 & 5.00335200 & 1.75178400 \\ \mathrm{H} & -3.02393500 & 5.63952300 & 0.87229000 \\ \mathrm{H} & -2.30499100 & 5.56157500 & 2.49287300 \\ \mathrm{H} & -3.87594800 & 4.81813300 & 2.19483700 \\ \mathrm{H} & -2.26864200 & 2.91562700 & 3.43772800 \\ \mathrm{H} & -1.10002500 & 0.82331500 & 2.84033200 \\ \mathrm{H} & -0.86423100 & 2.19640300 & -2.18154000\end{array}$

Migratory Insertion TS (TS1)

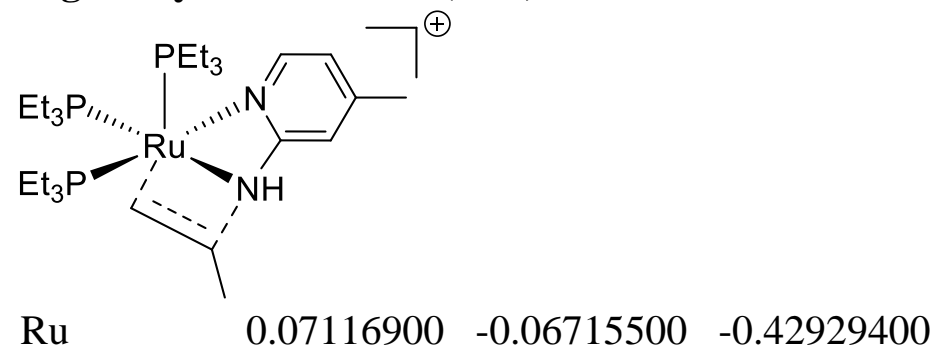




\begin{tabular}{|c|c|c|c|}
\hline $\mathrm{P}$ & 2.10440500 & -1.22477700 & -0.37085200 \\
\hline $\mathrm{P}$ & -0.40697100 & -0.31733700 & 1.88365700 \\
\hline $\mathrm{P}$ & 1.06451100 & 2.02978400 & -0.25046100 \\
\hline $\mathrm{N}$ & -2.08425100 & 0.42635200 & -0.56575500 \\
\hline $\mathrm{C}$ & -3.02086100 & 1.35290600 & -0.29386700 \\
\hline $\mathrm{H}$ & -2.66261700 & 2.34916900 & -0.06291100 \\
\hline $\mathrm{C}$ & -4.37639600 & 1.07204700 & -0.29752600 \\
\hline $\mathrm{H}$ & -5.09022000 & 1.85805900 & -0.07232000 \\
\hline $\mathrm{C}$ & -4.81601900 & -0.23693300 & -0.60005800 \\
\hline $\mathrm{C}$ & -6.28529800 & -0.56622200 & -0.61237800 \\
\hline $\mathrm{H}$ & -6.81155000 & 0.03630000 & -1.36250400 \\
\hline $\mathrm{H}$ & -6.74350900 & -0.34315300 & 0.35851900 \\
\hline $\mathrm{H}$ & -6.46066800 & -1.62163700 & -0.83912400 \\
\hline $\mathrm{C}$ & -3.85276300 & -1.19640400 & -0.88905100 \\
\hline $\mathrm{H}$ & -4.13505000 & -2.21900700 & -1.12437900 \\
\hline $\mathrm{C}$ & -2.48787200 & -0.84481200 & -0.85848000 \\
\hline $\mathrm{C}$ & 1.94797900 & -3.01170800 & 0.12987500 \\
\hline $\mathrm{H}$ & 2.94391000 & -3.40725700 & 0.34768000 \\
\hline $\mathrm{H}$ & 1.38729000 & -3.03706600 & 1.06751000 \\
\hline $\mathrm{C}$ & 1.26266500 & -3.90138500 & -0.91226100 \\
\hline $\mathrm{H}$ & 1.11096300 & -4.90847200 & -0.50901600 \\
\hline $\mathrm{H}$ & 1.86657200 & -4.00034200 & -1.81988000 \\
\hline $\mathrm{H}$ & 0.28790000 & -3.50382700 & -1.19988700 \\
\hline $\mathrm{C}$ & 3.45059300 & -0.56455500 & 0.75369300 \\
\hline $\mathrm{H}$ & 4.07090400 & 0.08985800 & 0.12822800 \\
\hline $\mathrm{H}$ & 2.95889400 & 0.08744800 & 1.47332600 \\
\hline $\mathrm{C}$ & 4.34040800 & -1.55722800 & 1.51182600 \\
\hline $\mathrm{H}$ & 4.88097900 & -2.23468300 & 0.84595200 \\
\hline $\mathrm{H}$ & 3.75824500 & -2.16613400 & 2.21086500 \\
\hline $\mathrm{H}$ & 5.08528200 & -1.00797500 & 2.09896000 \\
\hline $\mathrm{C}$ & 3.01724600 & -1.38264300 & -1.98981900 \\
\hline $\mathrm{H}$ & 2.32574900 & -1.84420900 & -2.70300100 \\
\hline $\mathrm{H}$ & 3.19058800 & -0.36436700 & -2.35448000 \\
\hline $\mathrm{C}$ & 4.34263600 & -2.15315500 & -1.97630500 \\
\hline $\mathrm{H}$ & 4.74922000 & -2.21199900 & -2.99216200 \\
\hline $\mathrm{H}$ & 4.22296300 & -3.17826800 & -1.61162100 \\
\hline $\mathrm{H}$ & 5.09337600 & -1.65805700 & -1.35326500 \\
\hline $\mathrm{C}$ & -1.09741400 & 1.26029900 & 2.63491800 \\
\hline $\mathrm{H}$ & -0.30572600 & 1.69137300 & 3.25889000 \\
\hline $\mathrm{H}$ & -1.23990600 & 1.95333800 & 1.80365600 \\
\hline $\mathrm{C}$ & -2.40075500 & 1.17045100 & 3.43727800 \\
\hline $\mathrm{H}$ & -2.29942400 & 0.54080900 & 4.32600300 \\
\hline $\mathrm{H}$ & -3.21940700 & 0.77263700 & 2.82973500 \\
\hline $\mathrm{H}$ & -2.69487500 & 2.17095800 & 3.77494900 \\
\hline $\mathrm{C}$ & 0.89535800 & -0.78892600 & 3.13278200 \\
\hline $\mathrm{H}$ & 1.37590300 & -1.70643900 & 2.77857400 \\
\hline
\end{tabular}




\begin{tabular}{|c|c|c|c|}
\hline $\mathrm{H}$ & 1.66750900 & -0.01399300 & 3.09516200 \\
\hline $\mathrm{C}$ & 0.42259900 & -0.96696400 & 4.58038500 \\
\hline $\mathrm{H}$ & -0.04524200 & -0.05703000 & 4.96964400 \\
\hline $\mathrm{H}$ & 1.27538800 & -1.19985400 & 5.22789300 \\
\hline $\mathrm{H}$ & -0.29712200 & -1.78503700 & 4.67900100 \\
\hline $\mathrm{C}$ & -1.72364000 & -1.58381000 & 2.23271000 \\
\hline $\mathrm{H}$ & -2.06467300 & -1.46117400 & 3.26437300 \\
\hline $\mathrm{H}$ & -2.57866500 & -1.34964100 & 1.59380200 \\
\hline $\mathrm{C}$ & -1.27273300 & -3.02936500 & 2.00277800 \\
\hline $\mathrm{H}$ & -2.11286800 & -3.71592300 & 2.15575000 \\
\hline $\mathrm{H}$ & -0.48185600 & -3.32428800 & 2.70117300 \\
\hline $\mathrm{H}$ & -0.89835600 & -3.17167000 & 0.98711400 \\
\hline $\mathrm{C}$ & 2.37092400 & 2.38142400 & -1.52967000 \\
\hline $\mathrm{H}$ & 3.16228200 & 1.64173300 & -1.36515100 \\
\hline $\mathrm{H}$ & 1.93415000 & 2.14667100 & -2.50525300 \\
\hline $\mathrm{C}$ & 2.97551000 & 3.78980400 & -1.54563000 \\
\hline $\mathrm{H}$ & 2.22291400 & 4.55634100 & -1.75499500 \\
\hline $\mathrm{H}$ & 3.73910300 & 3.86024400 & -2.32834300 \\
\hline $\mathrm{H}$ & 3.45816100 & 4.03917700 & -0.59534300 \\
\hline $\mathrm{C}$ & -0.11628400 & 3.44494200 & -0.51994200 \\
\hline $\mathrm{H}$ & -0.87698400 & 3.38953600 & 0.26571900 \\
\hline $\mathrm{H}$ & 0.42676600 & 4.37755900 & -0.34403600 \\
\hline $\mathrm{C}$ & -0.77461100 & 3.48867800 & -1.90170600 \\
\hline $\mathrm{H}$ & -1.37222200 & 2.59612400 & -2.09330200 \\
\hline $\mathrm{H}$ & -0.02986500 & 3.57481900 & -2.69984700 \\
\hline $\mathrm{H}$ & -1.43380000 & 4.36086500 & -1.97474300 \\
\hline $\mathrm{C}$ & 1.94799800 & 2.52821100 & 1.33238100 \\
\hline $\mathrm{H}$ & 1.65517900 & 1.80497700 & 2.09476100 \\
\hline $\mathrm{H}$ & 3.01682800 & 2.36451800 & 1.15398700 \\
\hline $\mathrm{C}$ & 1.72785300 & 3.94222500 & 1.88412400 \\
\hline $\mathrm{H}$ & 0.67506900 & 4.12754900 & 2.11974600 \\
\hline $\mathrm{H}$ & 2.05926900 & 4.71837900 & 1.18873200 \\
\hline $\mathrm{H}$ & 2.29924300 & 4.06643100 & 2.81110000 \\
\hline $\mathrm{N}$ & -1.40232200 & -1.62694800 & -1.08694300 \\
\hline $\mathrm{H}$ & -1.59325100 & -2.62208600 & -1.14613900 \\
\hline $\mathrm{C}$ & -0.62186800 & -1.09985600 & -2.97141800 \\
\hline $\mathrm{H}$ & -0.05967200 & -2.03090300 & -3.00551600 \\
\hline $\mathrm{C}$ & -1.90563100 & -1.09516400 & -3.74469200 \\
\hline $\mathrm{H}$ & -1.65290100 & -1.02124800 & -4.81230900 \\
\hline $\mathrm{H}$ & -2.49452900 & -2.00559500 & -3.60005500 \\
\hline $\mathrm{H}$ & -2.52120600 & -0.22588100 & -3.49453800 \\
\hline $\mathrm{C}$ & 0.10438200 & 0.08363800 & -2.72471100 \\
\hline $\mathrm{H}$ & -0.37249300 & 1.00741000 & -3.04066500 \\
\hline $\mathrm{H}$ & 1.16942500 & 0.04592000 & -2.91475700 \\
\hline
\end{tabular}

\section{Ru-alkyl Complex (33)}




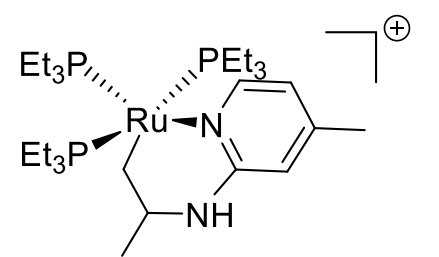

$\begin{array}{lccc}\mathrm{Ru} & -0.16285900 & -0.00105500 & -0.04158900 \\ \mathrm{P} & -2.46903900 & -0.38545800 & 0.09894800 \\ \mathrm{C} & -3.29195100 & -1.05758100 & -1.42954300 \\ \mathrm{H} & -2.74728800 & -1.95730000 & -1.72806300 \\ \mathrm{H} & -4.31167300 & -1.37352300 & -1.19057500 \\ \mathrm{C} & -3.30924400 & -0.05599400 & -2.58955500 \\ \mathrm{H} & -3.92349100 & 0.82018800 & -2.35673100 \\ \mathrm{H} & -2.30305700 & 0.29543100 & -2.83407500 \\ \mathrm{H} & -3.72810700 & -0.51959400 & -3.48916000 \\ \mathrm{C} & -3.57810800 & 1.05471200 & 0.51273400 \\ \mathrm{C} & -5.08651700 & 0.79451700 & 0.58975000 \\ \mathrm{H} & -5.61454000 & 1.72700200 & 0.81949900 \\ \mathrm{H} & -5.33580500 & 0.07742900 & 1.37718500 \\ \mathrm{H} & -5.48920500 & 0.41492700 & -0.35467700 \\ \mathrm{H} & -3.22447400 & 1.43039400 & 1.47829600 \\ \mathrm{H} & -3.37644800 & 1.84729800 & -0.21197800 \\ \mathrm{C} & -2.94885300 & -1.56512000 & 1.47711400 \\ \mathrm{H} & -2.03854300 & -2.08514600 & 1.77909300 \\ \mathrm{C} & -4.06499700 & -2.58193100 & 1.21203700 \\ \mathrm{H} & -3.80844400 & -3.26548800 & 0.39688000 \\ \mathrm{H} & -4.23279300 & -3.18960100 & 2.10870100 \\ \mathrm{H} & -5.01446100 & -2.10356700 & 0.95735000 \\ \mathrm{H} & -3.21830700 & -0.92979600 & 2.32970800 \\ \mathrm{P} & -0.32990300 & 2.37791200 & -0.13988600 \\ \mathrm{C} & -1.18071600 & 2.97795700 & -1.68285600 \\ \mathrm{H} & -2.19794200 & 2.57853100 & -1.68319300 \\ \mathrm{C} & -0.44788900 & 2.51699200 & -2.94865100 \\ \mathrm{H} & 0.54313400 & 2.97435400 & -3.03361800 \\ \mathrm{H} & -0.31554700 & 1.42881700 & -2.96024000 \\ \mathrm{H} & -1.01602200 & 2.78884400 & -3.84445100 \\ \mathrm{H} & -1.26795300 & 4.06830600 & -1.67221600 \\ \mathrm{C} & 1.31088500 & 3.25686700 & -0.26642100 \\ \mathrm{C} & 1.26560300 & 4.78004700 & -0.42772600 \\ \mathrm{H} & 0.75155200 & 5.08054400 & -1.34654700 \\ \mathrm{H} & 2.28439400 & 5.18074000 & -0.47864900 \\ \mathrm{H} & 0.76504100 & 5.26945100 & 0.41363500 \\ \mathrm{H} & 1.87343900 & 2.99002300 & 0.63519500 \\ \mathrm{H} & 1.86376600 & 2.81085000 & -1.09741400 \\ \mathrm{H} & -1.11192700 & 3.31414400 & 1.28381800 \\ & -2.09051700 & 4.44375900 & 0.94269200 \\ \mathrm{H} & -1.61619400 & 5.23736600 & 0.35836000\end{array}$




\begin{tabular}{|c|c|c|c|}
\hline $\mathrm{H}$ & -2.95088900 & 4.07795100 & 0.37346100 \\
\hline $\mathrm{H}$ & -2.47412200 & 4.89574900 & 1.86435600 \\
\hline $\mathrm{H}$ & -1.60491200 & 2.57796200 & 1.92209400 \\
\hline $\mathrm{H}$ & -0.27919400 & 3.71055900 & 1.87720900 \\
\hline $\mathrm{P}$ & 0.37256900 & -2.35707800 & -0.32009900 \\
\hline $\mathrm{C}$ & 1.85535800 & -3.06468900 & 0.55141100 \\
\hline $\mathrm{C}$ & 1.63734600 & -3.39135100 & 2.03201800 \\
\hline $\mathrm{H}$ & 0.84006200 & -4.12887500 & 2.17521200 \\
\hline $\mathrm{H}$ & 2.55190900 & -3.81284000 & 2.46415300 \\
\hline $\mathrm{H}$ & 1.38435600 & -2.49351600 & 2.60020400 \\
\hline $\mathrm{H}$ & 2.16275800 & -3.96426700 & 0.00467300 \\
\hline $\mathrm{H}$ & 2.66739300 & -2.34140200 & 0.43945500 \\
\hline $\mathrm{C}$ & 0.93363300 & -2.53177300 & -2.08519500 \\
\hline $\mathrm{H}$ & 1.11802400 & -3.58741800 & -2.31079900 \\
\hline $\mathrm{C}$ & -0.04263900 & -1.92333600 & -3.09568700 \\
\hline $\mathrm{H}$ & -0.25572900 & -0.87265500 & -2.86260900 \\
\hline $\mathrm{H}$ & 0.36561600 & -1.96376700 & -4.11144000 \\
\hline $\mathrm{H}$ & -1.00082100 & -2.45267500 & -3.10386900 \\
\hline $\mathrm{H}$ & 1.90479000 & -2.02988100 & -2.14769800 \\
\hline $\mathrm{C}$ & -0.86658900 & -3.73750800 & -0.13460400 \\
\hline $\mathrm{H}$ & -1.75851900 & -3.44342800 & -0.69506100 \\
\hline $\mathrm{C}$ & -0.42615400 & -5.14346900 & -0.55729500 \\
\hline $\mathrm{H}$ & -1.23831300 & -5.85658900 & -0.37651200 \\
\hline $\mathrm{H}$ & 0.44375600 & -5.48920200 & 0.00948500 \\
\hline $\mathrm{H}$ & -0.17907100 & -5.19558600 & -1.62212600 \\
\hline $\mathrm{H}$ & -1.16736000 & -3.74883800 & 0.91761800 \\
\hline $\mathrm{C}$ & -0.10531400 & 0.01980300 & 2.04238500 \\
\hline $\mathrm{C}$ & 1.13555600 & 0.68159500 & 2.62198000 \\
\hline $\mathrm{N}$ & 2.38522400 & 0.05751200 & 2.14725700 \\
\hline $\mathrm{C}$ & 2.86555400 & 0.15030400 & 0.86849500 \\
\hline $\mathrm{N}$ & 1.99995800 & 0.24902900 & -0.16238400 \\
\hline $\mathrm{C}$ & 2.51364300 & 0.35490700 & -1.41239400 \\
\hline $\mathrm{C}$ & 3.85893300 & 0.31742400 & -1.70363200 \\
\hline $\mathrm{C}$ & 4.77981700 & 0.16680500 & -0.64124400 \\
\hline $\mathrm{C}$ & 4.26299800 & 0.09309300 & 0.64037200 \\
\hline $\mathrm{H}$ & 4.92842800 & 0.00072000 & 1.49478800 \\
\hline $\mathrm{C}$ & 6.26004300 & 0.11230900 & -0.90520000 \\
\hline $\mathrm{H}$ & 6.83216400 & -0.00138800 & 0.01970800 \\
\hline $\mathrm{H}$ & 6.59871800 & 1.02608800 & -1.40790700 \\
\hline $\mathrm{H}$ & 6.50509000 & -0.72815700 & -1.56555400 \\
\hline $\mathrm{H}$ & 4.19610000 & 0.40229500 & -2.73165700 \\
\hline $\mathrm{H}$ & 1.77880300 & 0.46647400 & -2.20407300 \\
\hline $\mathrm{H}$ & 3.12004300 & 0.04430900 & 2.84364900 \\
\hline $\mathrm{C}$ & 1.14652200 & 0.61529100 & 4.15416900 \\
\hline $\mathrm{H}$ & 2.01591400 & 1.13562600 & 4.57740200 \\
\hline $\mathrm{H}$ & 0.24706700 & 1.09171600 & 4.55463300 \\
\hline
\end{tabular}




$\begin{array}{rrrr}\mathrm{H} & 1.16026200 & -0.42600900 & 4.49675700 \\ \mathrm{H} & 1.14603400 & 1.74182900 & 2.33102000 \\ \mathrm{H} & -0.97364500 & 0.55971500 & 2.44181200 \\ \mathrm{H} & -0.16747600 & -1.00764200 & 2.41685600\end{array}$

BHE TS (TS2)<smiles></smiles>

$\begin{array}{lccc}\mathrm{Ru} & -0.04283900 & -0.19725900 & -0.40840600 \\ \mathrm{P} & -2.37676400 & -0.46095000 & -0.62340700 \\ \mathrm{C} & -3.45019900 & 0.61561200 & 0.47526300 \\ \mathrm{H} & -2.82962200 & 0.89899600 & 1.32412900 \\ \mathrm{H} & -3.63449200 & 1.53796900 & -0.08743000 \\ \mathrm{C} & -4.78164200 & 0.06026900 & 0.99828400 \\ \mathrm{H} & -5.46137400 & -0.23732300 & 0.19635500 \\ \mathrm{H} & -4.63326800 & -0.80658900 & 1.64963300 \\ \mathrm{H} & -5.28815200 & 0.82992900 & 1.59186000 \\ \mathrm{C} & -3.06148300 & -2.16056300 & -0.27319600 \\ \mathrm{C} & -2.54040500 & -3.29246400 & -1.15790500 \\ \mathrm{H} & -2.81281200 & -3.14754300 & -2.20810200 \\ \mathrm{H} & -2.97472500 & -4.24596200 & -0.83767500 \\ \mathrm{H} & -1.45289600 & -3.37986200 & -1.09532100 \\ \mathrm{H} & -2.84022500 & -2.38831800 & 0.77320900 \\ \mathrm{H} & -4.15115100 & -2.10741700 & -0.34854300 \\ \mathrm{C} & -3.07519100 & -0.10674900 & -2.31508300 \\ \mathrm{H} & -2.72251800 & 0.89308100 & -2.58826500 \\ \mathrm{C} & -4.59486400 & -0.18890700 & -2.49603300 \\ \mathrm{H} & -5.11786700 & 0.55796700 & -1.89159100 \\ \mathrm{H} & -4.85382000 & 0.00032500 & -3.54386400 \\ \mathrm{H} & -4.99039700 & -1.17577000 & -2.23533900 \\ \mathrm{H} & -2.58632500 & -0.79296800 & -3.01246300 \\ \mathrm{P} & -0.08552100 & -1.16270200 & 1.80376900 \\ \mathrm{C} & -1.56384900 & -0.93556000 & 2.92723400 \\ \mathrm{H} & -1.65139200 & 0.14160000 & 3.10565200 \\ \mathrm{C} & -1.57269400 & -1.68221100 & 4.26647400 \\ \mathrm{H} & -0.71708800 & -1.41814400 & 4.89532200 \\ \mathrm{H} & -2.47965800 & -1.42746700 & 4.82658200 \\ \mathrm{H} & -1.56843200 & -2.76779300 & 4.13026200 \\ \mathrm{H} & -2.45345200 & -1.21047600 & 2.35652700 \\ \mathrm{C} & 1.28919800 & -0.79646600 & 3.01130100 \\ \mathrm{C} & 1.23778500 & 0.59570400 & 3.64858900 \\ \mathrm{H} & 2.12829600 & 0.77152300 & 4.26217300\end{array}$




\begin{tabular}{|c|c|c|c|}
\hline $\mathrm{H}$ & 0.36482400 & 0.71054200 & 4.29919800 \\
\hline $\mathrm{H}$ & 1.18605900 & 1.39215500 & 2.89961600 \\
\hline $\mathrm{H}$ & 1.25120400 & -1.56331800 & 3.79354100 \\
\hline $\mathrm{H}$ & 2.23744400 & -0.94533700 & 2.48943400 \\
\hline $\mathrm{C}$ & 0.06648800 & -3.01826400 & 1.73356200 \\
\hline $\mathrm{C}$ & 1.33528700 & -3.50693300 & 1.02626600 \\
\hline $\mathrm{H}$ & 1.38485800 & -3.13578500 & -0.00174800 \\
\hline $\mathrm{H}$ & 2.24137000 & -3.17767200 & 1.54654800 \\
\hline $\mathrm{H}$ & 1.35339400 & -4.60192900 & 0.99482600 \\
\hline $\mathrm{H}$ & 0.03695300 & -3.41284500 & 2.75501600 \\
\hline $\mathrm{H}$ & -0.81920800 & -3.39886800 & 1.21363200 \\
\hline $\mathrm{P}$ & -0.20971700 & 2.17516200 & 0.00797900 \\
\hline $\mathrm{C}$ & 1.35118500 & 3.19768800 & -0.07717000 \\
\hline $\mathrm{C}$ & 2.12310200 & 3.10371600 & -1.39641800 \\
\hline $\mathrm{H}$ & 2.98172800 & 3.78396100 & -1.37386400 \\
\hline $\mathrm{H}$ & 2.50141400 & 2.09555900 & -1.57390900 \\
\hline $\mathrm{H}$ & 1.50127500 & 3.38942300 & -2.25134800 \\
\hline $\mathrm{H}$ & 1.06586100 & 4.23713300 & 0.10512900 \\
\hline $\mathrm{H}$ & 2.00003100 & 2.91964700 & 0.75706500 \\
\hline $\mathrm{C}$ & -1.00558300 & 2.80422500 & 1.59463500 \\
\hline $\mathrm{H}$ & -1.01141800 & 1.96042600 & 2.28825100 \\
\hline $\mathrm{C}$ & -0.39757800 & 4.02420000 & 2.29776300 \\
\hline $\mathrm{H}$ & -0.42718300 & 4.92222100 & 1.67434400 \\
\hline $\mathrm{H}$ & -0.96280800 & 4.23965200 & 3.21178100 \\
\hline $\mathrm{H}$ & 0.64338500 & 3.85330200 & 2.59016800 \\
\hline $\mathrm{H}$ & -2.05489300 & 3.00879800 & 1.35517800 \\
\hline $\mathrm{C}$ & -1.23168300 & 3.03163800 & -1.29668900 \\
\hline $\mathrm{H}$ & -2.22418200 & 2.57443500 & -1.25420000 \\
\hline $\mathrm{C}$ & -1.36877100 & 4.55520200 & -1.21190400 \\
\hline $\mathrm{H}$ & -2.02843200 & 4.91626200 & -2.00921800 \\
\hline $\mathrm{H}$ & -0.40708300 & 5.06322900 & -1.33195700 \\
\hline $\mathrm{H}$ & -1.80401200 & 4.87388000 & -0.25909800 \\
\hline $\mathrm{H}$ & -0.80862000 & 2.73753300 & -2.26263300 \\
\hline $\mathrm{C}$ & 0.29958000 & 0.17391400 & -2.53473100 \\
\hline $\mathrm{C}$ & 0.62381800 & -1.21707400 & -2.37592000 \\
\hline $\mathrm{N}$ & 2.00440800 & -1.55489300 & -2.17529000 \\
\hline $\mathrm{C}$ & 2.80333100 & -0.89572100 & -1.28831600 \\
\hline $\mathrm{N}$ & 2.17150700 & -0.18181100 & -0.32771800 \\
\hline $\mathrm{C}$ & 2.95217000 & 0.44243900 & 0.58070700 \\
\hline $\mathrm{C}$ & 4.33048600 & 0.38530800 & 0.58971600 \\
\hline $\mathrm{C}$ & 4.99583900 & -0.35585900 & -0.40956700 \\
\hline $\mathrm{C}$ & 4.20785400 & -0.99329600 & -1.35555200 \\
\hline $\mathrm{H}$ & 4.66141500 & -1.56560800 & -2.15977800 \\
\hline $\mathrm{C}$ & 6.49769800 & -0.43431800 & -0.44556600 \\
\hline $\mathrm{H}$ & 6.88478800 & -0.86976800 & 0.48335000 \\
\hline $\mathrm{H}$ & 6.93618000 & 0.56620700 & -0.54008800 \\
\hline
\end{tabular}




$\begin{array}{lrrr}\mathrm{H} & 6.85142200 & -1.04331400 & -1.28202100 \\ \mathrm{H} & 4.88595000 & 0.91169200 & 1.35932100 \\ \mathrm{H} & 2.42146300 & 1.00515300 & 1.33114000 \\ \mathrm{H} & 2.44341800 & -2.15323200 & -2.86168900 \\ \mathrm{C} & -0.04331200 & -2.26792400 & -3.24159300 \\ \mathrm{H} & -1.10670400 & -2.06929800 & -3.35508900 \\ \mathrm{H} & 0.40555100 & -2.22625700 & -4.24297200 \\ \mathrm{H} & 0.08457600 & -3.27729000 & -2.84022500 \\ \mathrm{H} & 0.04674800 & -1.77551600 & -0.98211200 \\ \mathrm{H} & -0.58020900 & 0.41081900 & -3.12265700 \\ \mathrm{H} & 1.12895800 & 0.85633100 & -2.68515100\end{array}$

\section{Ru-hydridoenamine Complex (45)}

$\begin{array}{lrrr} & & \\ \end{array}$




\begin{tabular}{|c|c|c|c|}
\hline $\mathrm{C}$ & -1.25098100 & 2.55837800 & -2.77098200 \\
\hline $\mathrm{H}$ & -1.55900500 & 1.50483100 & -2.75656900 \\
\hline $\mathrm{H}$ & -0.18878300 & 2.59394600 & -3.03899500 \\
\hline $\mathrm{H}$ & -1.80863200 & 3.05978400 & -3.56935300 \\
\hline $\mathrm{C}$ & -1.30533000 & 3.09866800 & 1.49655300 \\
\hline $\mathrm{H}$ & -1.42335600 & 4.17857800 & 1.34886500 \\
\hline $\mathrm{H}$ & -2.30897100 & 2.68568600 & 1.62819300 \\
\hline $\mathrm{C}$ & -0.46281000 & 2.81333900 & 2.74474400 \\
\hline $\mathrm{H}$ & 0.52744500 & 3.27526200 & 2.68452400 \\
\hline $\mathrm{H}$ & -0.31851100 & 1.73804300 & 2.88571600 \\
\hline $\mathrm{H}$ & -0.95857800 & 3.20586500 & 3.63881500 \\
\hline $\mathrm{C}$ & -3.93662600 & 1.04112500 & -0.32438700 \\
\hline $\mathrm{H}$ & -3.64099400 & 1.87864600 & 0.31221600 \\
\hline $\mathrm{H}$ & -3.71521300 & 1.34720500 & -1.35206500 \\
\hline $\mathrm{C}$ & -5.44008500 & 0.78638900 & -0.16779900 \\
\hline $\mathrm{H}$ & -5.79758000 & -0.00344900 & -0.83536700 \\
\hline $\mathrm{H}$ & -5.99816100 & 1.69669900 & -0.41408500 \\
\hline $\mathrm{H}$ & -5.70497300 & 0.50929800 & 0.85730500 \\
\hline $\mathrm{C}$ & -3.38128500 & -0.92819900 & 1.72111200 \\
\hline $\mathrm{H}$ & -4.43890200 & -1.20045200 & 1.63079500 \\
\hline $\mathrm{H}$ & -2.84193900 & -1.84521200 & 1.96825300 \\
\hline $\mathrm{C}$ & -3.18448800 & 0.10317700 & 2.83497900 \\
\hline $\mathrm{H}$ & -2.13181000 & 0.38384700 & 2.92815700 \\
\hline $\mathrm{H}$ & -3.51282400 & -0.30554000 & 3.79657400 \\
\hline $\mathrm{H}$ & -3.76393300 & 1.01476200 & 2.65200500 \\
\hline $\mathrm{C}$ & -3.49070000 & -1.68795200 & -1.04657900 \\
\hline $\mathrm{H}$ & -3.01409800 & -2.63448900 & -0.77634000 \\
\hline $\mathrm{H}$ & -4.55627100 & -1.80903100 & -0.82408900 \\
\hline $\mathrm{C}$ & -3.28201300 & -1.38549400 & -2.53420100 \\
\hline $\mathrm{H}$ & -2.22686600 & -1.19019100 & -2.75682200 \\
\hline $\mathrm{H}$ & -3.85565400 & -0.50729500 & -2.84874800 \\
\hline $\mathrm{H}$ & -3.60468000 & -2.22754200 & -3.15602000 \\
\hline $\mathrm{C}$ & -0.79213900 & -3.44213100 & 1.08242000 \\
\hline $\mathrm{H}$ & -1.87487500 & -3.39551500 & 0.92960300 \\
\hline $\mathrm{H}$ & -0.50383300 & -4.48392600 & 0.90233600 \\
\hline $\mathrm{C}$ & -0.43092500 & -3.02435300 & 2.51239200 \\
\hline $\mathrm{H}$ & 0.63713800 & -3.15624200 & 2.71262800 \\
\hline $\mathrm{H}$ & -0.98176800 & -3.62871600 & 3.24124600 \\
\hline $\mathrm{H}$ & -0.66810400 & -1.97109700 & 2.69126600 \\
\hline $\mathrm{C}$ & 1.81346700 & -2.74110400 & 0.07689700 \\
\hline $\mathrm{H}$ & 2.36842000 & -2.23567200 & -0.71887500 \\
\hline $\mathrm{H}$ & 2.08592700 & -2.23301700 & 1.00632800 \\
\hline $\mathrm{C}$ & 2.22675200 & -4.21491000 & 0.14682100 \\
\hline $\mathrm{H}$ & 2.00450100 & -4.75291400 & -0.78012200 \\
\hline $\mathrm{H}$ & 1.73711700 & -4.74320300 & 0.97076100 \\
\hline $\mathrm{H}$ & 3.30778200 & -4.28757700 & 0.31203100 \\
\hline
\end{tabular}




$\begin{array}{lrrr}\mathrm{C} & -0.33119000 & -3.34674300 & -1.76064100 \\ \mathrm{H} & -0.00860100 & -4.38190800 & -1.60298300 \\ \mathrm{H} & -1.41423000 & -3.37931300 & -1.90776700 \\ \mathrm{C} & 0.34621500 & -2.75820800 & -3.00240500 \\ \mathrm{H} & 0.12654000 & -3.36616200 & -3.88642100 \\ \mathrm{H} & 1.43480600 & -2.71346800 & -2.89160600 \\ \mathrm{H} & -0.00972200 & -1.74194300 & -3.20428300 \\ \mathrm{H} & -0.44356700 & -0.08614500 & 1.34867700 \\ \mathrm{H} & 1.25988300 & 0.21067400 & 1.55972600 \\ \mathrm{C} & 2.94979200 & -0.10148300 & 2.67664900 \\ \mathrm{C} & 2.21842300 & -0.26464100 & 3.79445700 \\ \mathrm{H} & 1.14462800 & -0.10110000 & 3.80858400 \\ \mathrm{H} & 2.68881000 & -0.55967400 & 4.72420000 \\ \mathrm{C} & 4.43569700 & -0.33238700 & 2.65878400 \\ \mathrm{H} & 4.72873000 & -1.04329900 & 1.87792400 \\ \mathrm{H} & 4.99444500 & 0.59722300 & 2.49910600 \\ \mathrm{H} & 4.74659200 & -0.74079100 & 3.62251300\end{array}$

Ru-hydrido-aminopyridine Complex (46)

$\begin{array}{lccc} & & \\ \end{array}$




$\begin{array}{lrrr}\mathrm{H} & 2.23372800 & 5.14074400 & 0.23936100 \\ \mathrm{H} & 0.65343600 & 5.13406500 & 1.02262800 \\ \mathrm{H} & 0.77093900 & 5.19587500 & -0.74166200 \\ \mathrm{C} & -1.05465300 & 3.06352400 & -1.63246800 \\ \mathrm{H} & -2.14639200 & 3.03735800 & -1.60039500 \\ \mathrm{H} & -0.77134700 & 4.11828900 & -1.70850000 \\ \mathrm{C} & -0.53707600 & 2.27432500 & -2.84126300 \\ \mathrm{H} & -0.83296700 & 1.21892300 & -2.77502500 \\ \mathrm{H} & 0.55633900 & 2.31293800 & -2.90719200 \\ \mathrm{H} & -0.93776900 & 2.67595500 & -3.77827600 \\ \mathrm{C} & -1.40756200 & 3.19932800 & 1.26799900 \\ \mathrm{H} & -1.50838100 & 4.25925300 & 1.00649000 \\ \mathrm{H} & -2.41281400 & 2.77040900 & 1.24478100 \\ \mathrm{C} & -0.81372000 & 3.04345700 & 2.67244100 \\ \mathrm{H} & 0.15960000 & 3.53603500 & 2.75960000 \\ \mathrm{H} & -0.67556700 & 1.98782900 & 2.92586600 \\ \mathrm{H} & -1.47993300 & 3.48648100 & 3.42028100 \\ \mathrm{C} & -3.64321900 & 0.94749400 & -0.80864700 \\ \mathrm{H} & -3.46181700 & 1.83215000 & -0.19375000 \\ \mathrm{H} & -3.26385900 & 1.18884300 & -1.80708800 \\ \mathrm{C} & -5.14972800 & 0.67222700 & -0.87520200 \\ \mathrm{H} & -5.38857700 & -0.16641600 & -1.53616100 \\ \mathrm{H} & -5.67088900 & 1.55287400 & -1.26720600 \\ \mathrm{H} & -5.57139800 & 0.45655000 & 0.11144000 \\ \mathrm{C} & -3.40624900 & -0.88096500 & 1.42471700 \\ \mathrm{H} & -4.42775500 & -1.19564000 & 1.18297400 \\ \mathrm{H} & -2.89184900 & -1.76007100 & 1.82024000 \\ \mathrm{H} & -3.41986500 & 0.22722600 & 2.48062400 \\ \mathrm{C} & -2.40340700 & 0.54828800 & 2.72376000 \\ \mathrm{H} & -3.89362100 & -0.12809400 & 3.40201500 \\ \mathrm{H} & -3.98130300 & 1.10477400 & 2.14171400 \\ \mathrm{H} & -3.06276200 & -1.80748100 & -1.27036300 \\ \mathrm{C} & -2.62134000 & -2.72554800 & -0.87338400 \\ \mathrm{H} & -4.14807400 & -1.93916700 & -1.20475100 \\ \mathrm{H} & -2.63492800 & -1.58976800 & -2.72539600 \\ \mathrm{C} & -1.56221500 & -1.37917600 & -2.79860800 \\ \mathrm{H} & -3.16973600 & -0.74845900 & -3.17843300 \\ \mathrm{H} & -2.84427000 & -2.47641000 & -3.33362900 \\ \mathrm{H} & -0.71641300 & -3.40542900 & 1.33292100 \\ \mathrm{H} & -1.76236000 & -3.37498900 & 1.01250900 \\ \mathrm{H} & -0.58831900 & -2.93534400 & 2.78612700 \\ \mathrm{H} & -3452300 & -3.06065900 & 3.15728200 \\ \mathrm{H} & -3.51577300 & 3.43982100 \\ \mathrm{H} & -1.87743300 & 2.88589100 \\ \mathrm{H} & -2.69463400 & 0.72209100\end{array}$




$\begin{array}{lrrr}\mathrm{H} & 2.67804000 & -2.23535900 & -0.01373900 \\ \mathrm{H} & 2.14345300 & -2.12016800 & 1.64436700 \\ \mathrm{C} & 2.41562600 & -4.15491500 & 0.95081200 \\ \mathrm{H} & 2.32977300 & -4.75547700 & 0.03973000 \\ \mathrm{H} & 1.81425100 & -4.63488200 & 1.72912300 \\ \mathrm{H} & 3.46223100 & -4.20301600 & 1.27242000 \\ \mathrm{C} & 0.19094300 & -3.40979000 & -1.40026300 \\ \mathrm{H} & 0.50573700 & -4.42971900 & -1.15286900 \\ \mathrm{H} & -0.85571000 & -3.47576600 & -1.70930700 \\ \mathrm{C} & 1.03868600 & -2.85259800 & -2.54839200 \\ \mathrm{H} & 0.96202600 & -3.49577800 & -3.43144200 \\ \mathrm{H} & 2.09774100 & -2.78411800 & -2.27826000 \\ \mathrm{H} & 0.70313100 & -1.85111800 & -2.84027100 \\ \mathrm{H} & -0.46250300 & -0.01980400 & 1.51787900 \\ \mathrm{H} & 1.17339600 & 0.26374500 & 2.26636400 \\ \mathrm{H} & 2.71861200 & 0.42639100 & 3.04126600\end{array}$

\section{X-ray Structure of Ruthenium Complexes}

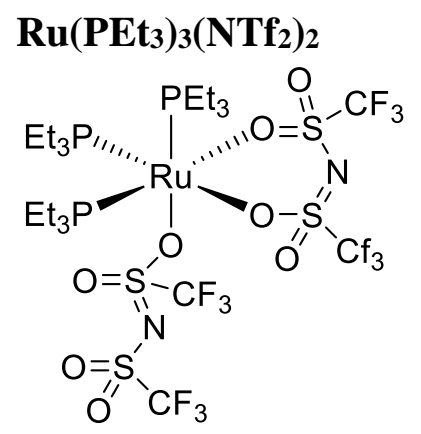

A yellow prism $0.060 \times 0.050 \times 0.050 \mathrm{~mm}$ in size was mounted on a Cryoloop with Paratone oil. Data were collected in a nitrogen gas stream at 100(2) K using and scans. Crystal-to-detector distance was $50 \mathrm{~mm}$ and exposure time was 10 seconds per frame using a scan width of $1.0^{\circ}$. Data collection was $100.0 \%$ complete to $25.000^{\circ}$ in $\theta$. A total of 122543 reflections were collected covering the indices, $-13<=h<=13,-22<=k<=22,-21<=l<=21.7199$ reflections were found to be symmetry independent, with an $\mathrm{R}_{\text {int }}$ of 0.0671 . Indexing and unit cell refinement indicated a primitive, monoclinic lattice. The space group was found to be P 21/c (No. 14). The data were integrated using the Bruker SAINT software program and scaled using the SADABS software program. Solution by iterative methods (SHELXT-2014) produced a complete heavyatom phasing model consistent with the proposed structure. All non-hydrogen atoms were refined anisotropically by full-matrix least-squares (SHELXL-2016). All hydrogen atoms were placed using a riding model. Their positions were constrained relative to their parent atom using the appropriate HFIX command in SHELXL-2016.

Table 1. Crystal data and structure refinement for $\mathrm{Ru}\left(\mathrm{PEt}_{3}\right)_{3}\left(\mathrm{NTf}_{2}\right)_{2}$.

Empirical formula

Formula weight
C22 H45.25 F12 N2 O8.25 P3 Ru S4

1020.14 
Temperature

Wavelength

Crystal system

Space group

Unit cell dimensions

Volume

$\mathrm{Z}$

Density (calculated)

Absorption coefficient

$\mathrm{F}(000)$

Crystal size

Theta range for data collection

Index ranges

Reflections collected

Independent reflections

Completeness to theta $=25.000^{\circ}$

Absorption correction

Max. and min. transmission

Refinement method

Data / restraints / parameters

Goodness-of-fit on $\mathrm{F}^{2}$

Final $\mathrm{R}$ indices [I $>2 \operatorname{sigma}(\mathrm{I})]$

$\mathrm{R}$ indices (all data)

Extinction coefficient

Largest diff. peak and hole
100(2) K

$0.71073 \AA$

Monoclinic

P 21/c

$\mathrm{a}=11.433(3) \AA \quad \alpha=90^{\circ}$.

$\mathrm{b}=18.944(5) \AA \quad \beta=91.423(5)^{\circ}$.

$\mathrm{c}=18.126(5) \AA \quad \gamma=90^{\circ}$.

3924.6(18) $\AA^{3}$

4

$1.727 \mathrm{Mg} / \mathrm{m}^{3}$

$0.836 \mathrm{~mm}^{-1}$

2073

$0.060 \times 0.050 \times 0.050 \mathrm{~mm}^{3}$

1.555 to $25.384^{\circ}$.

$-13<=\mathrm{h}<=13,-22<=\mathrm{k}<=22,-21<=\mathrm{l}<=21$

122543

$7199[\mathrm{R}(\mathrm{int})=0.0671]$

$100.0 \%$

Semi-empirical from equivalents

0.928 and 0.836

Full-matrix least-squares on $\mathrm{F}^{2}$

7199 / 4 / 468

1.092

$\mathrm{R} 1=0.0419, \mathrm{wR} 2=0.0933$

$\mathrm{R} 1=0.0468, \mathrm{wR} 2=0.0965$

$\mathrm{n} / \mathrm{a}$

1.150 and -0.993 e. $\AA^{-3}$

\section{Ru(PEt $\left.)_{3}\right)_{3}$ (Amine)(NTf $)_{2}$ (29)}

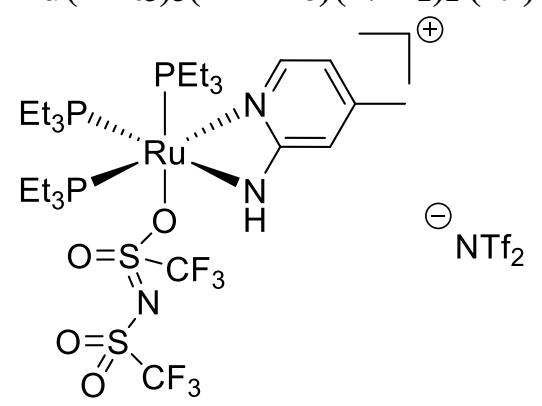

A yellow block $0.24 \times 0.10 \times 0.09 \mathrm{~mm}$ in size was mounted on a Cryoloop with Paratone oil. Data were collected in a nitrogen gas stream at 100(2) K using omega scans. Crystal-to-detector distance was $35 \mathrm{~mm}$ and exposure time was 6.00 seconds per frame using a scan width of $0.5^{\circ}$. Data collection was $100 \%$ complete to $26.372^{\circ}$ in $\theta$. A total of 72140 reflections were collected covering the indices $-13<=\mathrm{h}<=13,-22<=\mathrm{k}<=23,-28<=\mathrm{k}<=30.9834$ reflections were founded to be symmetry independent, with an $\mathrm{R}_{\text {int }}$ of 0.0536 . Indexing and unit cell refinement indicated a primitive, monoclinic lattice. The space group was found to be P 21/c (No. 14). The data were integrated using the CrysAlis ${ }^{\text {Pro }}$ 1.171.40.44a software program and scaled using the SCALE3 
ABSPACK scaling algorithm. Solution by intrinsic phasing (SHELXT-2015) produced a heavyatom phasing model consistent with the proposed structure. All non-hydrogen atoms were refined anisotropically by full-matrix least-squares (SHELXL-2014). All hydrogen atoms were placed using a riding model. Their positions were constrained relative to their parent atom using the appropriate HFIX command in SHELXL-2014.

Table 1. Crystal data and structure refinement for $\mathrm{Ru}\left(\mathrm{PEt}_{3}\right)_{3}(\mathrm{Amine})\left(\mathrm{NTf}_{2}\right)_{2}$.

Empirical formula

Formula weight

Temperature

Wavelength

Crystal system

Space group

Unit cell dimensions

Volume

$\mathrm{Z}$

Density (calculated)

Absorption coefficient

$\mathrm{F}(000)$

Crystal size

Theta range for data collection

Index ranges

Reflections collected

Independent reflections

Completeness to theta $=26.372^{\circ}$

Absorption correction

Max. and min. transmission

Refinement method

Data / restraints / parameters

Goodness-of-fit on $\mathrm{F}^{2}$

Final $\mathrm{R}$ indices [I $>2 \operatorname{sigma}(\mathrm{I})]$

$\mathrm{R}$ indices (all data)

Extinction coefficient

Largest diff. peak and hole
C29 H55 Cl2 F12 N4 O8 P3 Ru S4

1208.89

$100(2) \mathrm{K}$

$0.71073 \AA$

Monoclinic

P 21/c

$\mathrm{a}=10.5702(2) \AA$

$\alpha=90^{\circ}$.

$\mathrm{b}=18.7348(4) \AA$

$\mathrm{c}=24.3472(5) \AA$

$\beta=92.934(2)^{\circ}$.

$\gamma=90^{\circ}$.
4815.17(17) $\AA^{3}$

4

$1.668 \mathrm{Mg} / \mathrm{m}^{3}$

$0.804 \mathrm{~mm}^{-1}$

2464

$0.240 \times 0.100 \times 0.090 \mathrm{~mm}^{3}$

2.738 to $26.372^{\circ}$.

$-13<=\mathrm{h}<=13,-22<=\mathrm{k}<=23,-28<=\mathrm{l}<=30$

72140

$9834[\mathrm{R}(\mathrm{int})=0.0536]$

$99.8 \%$

Semi-empirical from equivalents

1.00000 and 0.55675

Full-matrix least-squares on $\mathrm{F}^{2}$

9834 / 2 / 632

1.029

$\mathrm{R} 1=0.0316, \mathrm{wR} 2=0.0730$

$\mathrm{R} 1=0.0378, \mathrm{wR} 2=0.0754$

$\mathrm{n} / \mathrm{a}$

0.617 and -1.025 e. $\AA^{-3}$

\section{Ru(PEt $\left.)_{3}\right)_{3}$ (Amido)(NTf $)_{2}$ (30)}

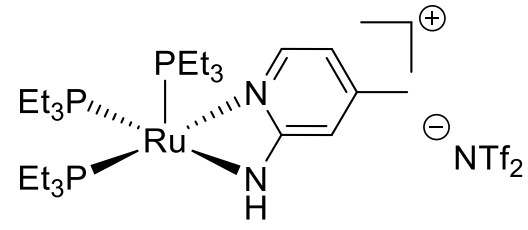

A red block $0.25 \times 0.11 \times 0.09 \mathrm{~mm}$ in size was mounted on a Cryoloop with Paratone oil. Data were collected in a nitrogen gas stream at 100(2) K using omega scans. Crystal-to-detector 
distance was $35 \mathrm{~mm}$ and exposure time was 2.00 seconds per frame using a scan width of $0.5^{\circ}$. Data collection was $100 \%$ complete to $26.369^{\circ}$ in $\theta$. A total of 49254 reflections were collected covering the indices $-15<=\mathrm{h}<=15,-17<=\mathrm{k}<=17,-25<=1<=25.7366$ reflections were founded to be symmetry independent, with an $\mathrm{R}_{\text {int }}$ of 0.0659 . Indexing and unit cell refinement indicated a primitive, monoclinic lattice. The space group was found to be $\mathrm{P} 21 / \mathrm{n}$ (No. 14). The data were integrated using the CrysAlis ${ }^{\text {Pro }}$ 1.171.40.44a software program and scaled using the SCALE3 ABSPACK scaling algorithm. Solution by intrinsic phasing (SHELXT-2015) produced a heavyatom phasing model consistent with the proposed structure. All non-hydrogen atoms were refined anisotropically by full-matrix least-squares (SHELXL-2014). All hydrogen atoms were placed using a riding model. Their positions were constrained relative to their parent atom using the appropriate HFIX command in SHELXL-2014.

Table 1. Crystal data and structure refinement for $\mathrm{Ru}\left(\mathrm{PEt}_{3}\right)_{3}(\mathrm{Amido})\left(\mathrm{NTf}_{2}\right)$.

Empirical formula

Formula weight

Temperature

Wavelength

Crystal system

Space group

Unit cell dimensions

Volume

Z

Density (calculated)

Absorption coefficient

$\mathrm{F}(000)$

Crystal size

Theta range for data collection

Index ranges

Reflections collected

Independent reflections

Completeness to theta $=26.369^{\circ}$

Absorption correction

Max. and min. transmission

Refinement method

Data / restraints / parameters

Goodness-of-fit on $\mathrm{F}^{2}$
C26 H52 F6 N3 O4 P3 Ru S2

842.80

100(2) K

$0.71073 \AA$

Monoclinic

P 21/n

$\mathrm{a}=12.6581(4) \AA$ $\alpha=90^{\circ}$.

$\mathrm{b}=14.3288(4) \AA$ $\beta=106.345(3)^{\circ}$.

$\mathrm{c}=20.7537(6) \AA$ $\gamma=90^{\circ}$.
3612.08(19) $\AA^{3}$

4

$1.550 \mathrm{Mg} / \mathrm{m}^{3}$

$0.749 \mathrm{~mm}^{-1}$

1744

$0.250 \times 0.110 \times 0.090 \mathrm{~mm}^{3}$

2.843 to $26.369^{\circ}$.

$-15<=\mathrm{h}<=15,-17<=\mathrm{k}<=17,-25<=\mathrm{l}<=25$

49254

$7366[\mathrm{R}(\mathrm{int})=0.0659]$

$99.9 \%$

Semi-empirical from equivalents

1.00000 and 0.28562

Full-matrix least-squares on $\mathrm{F}^{2}$

7366 / 6 / 440

1.033 
Final R indices [I $>2 \operatorname{sigma}(\mathrm{I})]$

$\mathrm{R}$ indices (all data)

Extinction coefficient

Largest diff. peak and hole
$\mathrm{R} 1=0.0335, \mathrm{wR} 2=0.0794$

$\mathrm{R} 1=0.0401, \mathrm{wR} 2=0.0819$

$\mathrm{n} / \mathrm{a}$

0.719 and -0.511 e. $\AA^{-3}$

\section{$\operatorname{Ru}(\mathbf{H})\left(\text { PEt3 }_{3}\right)_{3}($ Amine $)\left(\text { NTf }_{2}\right)_{2}$ (44)}<smiles>[H][R](CC)(CC)[n+]1ccc(C)cc1N</smiles>

A colorless block 0.31 x 0.17 x $0.10 \mathrm{~mm}$ in size was mounted on a Cryoloop with Paratone oil. Data were collected in a nitrogen gas stream at 100(2) K using omega scans. Crystal-to-detector distance was $33.00 \mathrm{~mm}$ and exposure time was 4.00 seconds per frame using a scan width of $0.5^{\circ}$. Data collection was $100 \%$ complete to $30.500^{\circ}$ in $\theta$. A total of 60357 reflections were collected covering the indices $-14<=\mathrm{h}<=14,-16<=\mathrm{k}<=16,-23<=\mathrm{l}<=23.11552$ reflections were found to be symmetry independent, with an $\mathrm{R}_{\text {int }}$ of 0.0955 . Indexing and unit cell refinement indicated a primitive, triclinic lattice. The space group was found to be P-1 (No. 2). The data were integrated using the CrysAlis ${ }^{\text {Pro }}$ 1.171.40.54a software program and scaled using the SCALE3 ABSPACK scaling algorithm. Solution by intrinsic phasing (SHELXT-2015) produced a heavyatom phasing model consistent with the proposed structure. All non-hydrogen atoms were refined anisotropically by full-matrix least-squares (SHELXL-2014). All hydrogen atoms were placed using a riding model. Their positions were constrained relative to their parent atom using the appropriate HFIX command in SHELXL-2014.

Table 1. Crystal data and structure refinement for $\mathrm{Ru}(\mathrm{H})\left(\mathrm{PEt}_{3}\right)_{3}(\mathrm{Amine})\left(\mathrm{NTf}_{2}\right)_{2}$.

Empirical formula

Formula weight

Temperature

Wavelength

Crystal system

Space group

Unit cell dimensions

Volume

\section{$\mathrm{Z}$}

Density (calculated)

Absorption coefficient

$\mathrm{F}(000)$
C26 H54 F6 N3.80 O4 P3 Ru S2

856.03

100(2) K

$0.71073 \AA$

Triclinic

$\mathrm{P}-1$

$\mathrm{a}=10.2586(2) \AA \quad \alpha=79.6870(10)^{\circ}$.

$\mathrm{b}=11.4779(2) \AA \quad \beta=82.7900(10)^{\circ}$.

$\mathrm{c}=16.7535(2) \AA \quad \gamma=78.3830(10)^{\circ}$.

2

$1.502 \mathrm{Mg} / \mathrm{m}^{3}$

$0.716 \mathrm{~mm}^{-1}$

887 
Crystal size

Theta range for data collection

Index ranges

Reflections collected

Independent reflections

Completeness to theta $=30.500^{\circ}$

Absorption correction

Max. and min. transmission

Refinement method

Data / restraints / parameters

Goodness-of-fit on $\mathrm{F}^{2}$

Final R indices [I $>2 \operatorname{sigma}(\mathrm{I})]$

$\mathrm{R}$ indices (all data)

Extinction coefficient

Largest diff. peak and hole
$0.310 \times 0.170 \times 0.100 \mathrm{~mm}^{3}$

2.979 to $30.508^{\circ}$.

$-14<=\mathrm{h}<=14,-16<=\mathrm{k}<=16,-23<=\mathrm{l}<=23$

60357

$11552[\mathrm{R}(\mathrm{int})=0.0955]$

$99.9 \%$

Semi-empirical from equivalents

1.00000 and 0.78072

Full-matrix least-squares on $\mathrm{F}^{2}$

11552 / $1 / 555$

1.055

$\mathrm{R} 1=0.0310, \mathrm{wR} 2=0.0736$

$\mathrm{R} 1=0.0392, \mathrm{wR} 2=0.0765$

$\mathrm{n} / \mathrm{a}$

0.684 and -0.750 e. $\AA^{-3}$

\section{References}

1. Evans, I. P.; Spencer, A.; Wilkinson, G., Dichlorotetrakis(dimethyl sulphoxide)ruthenium(II) and its use as a source material for some new ruthenium(II) complexes. J. Chem. Soc., Dalton Trans. 1973, (2), 204-209.

2. Hill, C. K.; Hartwig, J. F., Site-selective oxidation, amination and epimerization reactions of complex polyols enabled by transfer hydrogenation. Nat. Chem. 2017, 9 (12), 1213-1221.

3. Bannwarth, C.; Ehlert, S.; Grimme, S., GFN2-xTB-An Accurate and Broadly Parametrized Self-Consistent Tight-Binding Quantum Chemical Method with Multipole Electrostatics and Density-Dependent Dispersion Contributions. J. Chem. Theory Comput. 2019, 15 (3), 1652-1671.

4. Bursch, M.; Neugebauer, H.; Grimme, S., Structure Optimisation of Large TransitionMetal Complexes with Extended Tight-Binding Methods. Angew. Chem. Int. Ed. 2019, 58 (32), 11078-11087.

5. Grimme, S., Exploration of Chemical Compound, Conformer, and Reaction Space with Meta-Dynamics Simulations Based on Tight-Binding Quantum Chemical Calculations. J. Chem. Theory Comput. 2019, 15 (5), 2847-2862.

6. Grimme, S. grimme-lab / xtb. https://github.com/grimme-lab/xtb/releases.

7. M. J. Frisch, G. W. T., H. B. Schlegel, G. E. Scuseria, M. A.; Robb, J. R. C., G. Scalmani, V. Barone, G. A. Petersson, H. Nakatsuji, X. Li, M.; Caricato, A. V. M., J. Bloino, B. G. Janesko, R. Gomperts, B. Mennucci, H. P. Hratchian,; J. V. Ortiz, A. F. I., J. L. Sonnenberg, D. WilliamsYoung, F. Ding, F. Lipparini, F. Egidi, J.; Goings, B. P., A. Petrone, T. Henderson, D. Ranasinghe, V. G. Zakrzewski, J. Gao, N. Rega,; G. Zheng, W. L., M. Hada, M. Ehara, K. Toyota, R. Fukuda, J. Hasegawa, M. Ishida, T.; Nakajima, Y. H., O. Kitao, H. Nakai, T. Vreven, K. Throssell, J. A. Montgomery, Jr., J. E.; Peralta, F. O., M. J. Bearpark, J. J. Heyd, E. N. Brothers, K. N. Kudin, V. N. Staroverov, T.; A. Keith, R. K., J. Normand, K. Raghavachari, A. P. 
Rendell, J. C. Burant, S. S. Iyengar,; J. Tomasi, M. C., J. M. Millam, M. Klene, C. Adamo, R. Cammi, J. W. Ochterski, R. L.; Martin, K. M., O. Farkas, J. B. Foresman, D. J. Fox, Gaussian 16, Revision A.03. 2016.

8. Paton, R. S. GoodVibes v3.0.1 2019. https://github.com/bobbypaton/GoodVibes.

9. Marenich, A. V.; Cramer, C. J.; Truhlar, D. G., Universal Solvation Model Based on Solute Electron Density and on a Continuum Model of the Solvent Defined by the Bulk Dielectric Constant and Atomic Surface Tensions. J. Phys. Chem. B 2009, 113 (18), 6378-6396.

\section{Copies of Spectroscopy Data} cis- $\mathrm{Ru}\left(\mathrm{PMe}_{3}\right)_{4} \mathrm{Cl}_{2}$

${ }^{1} \mathbf{H}$

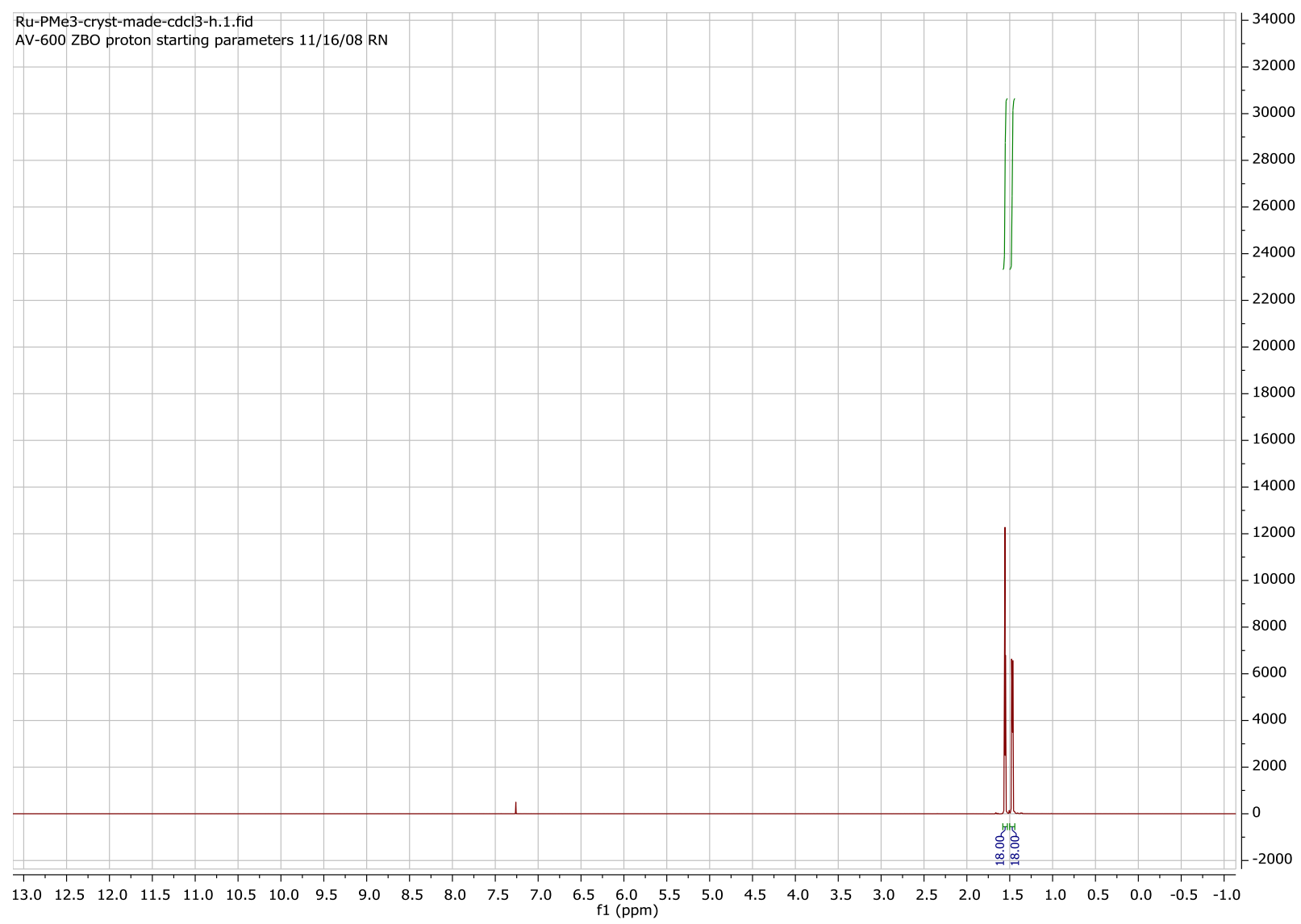


${ }^{31} \mathbf{P}$

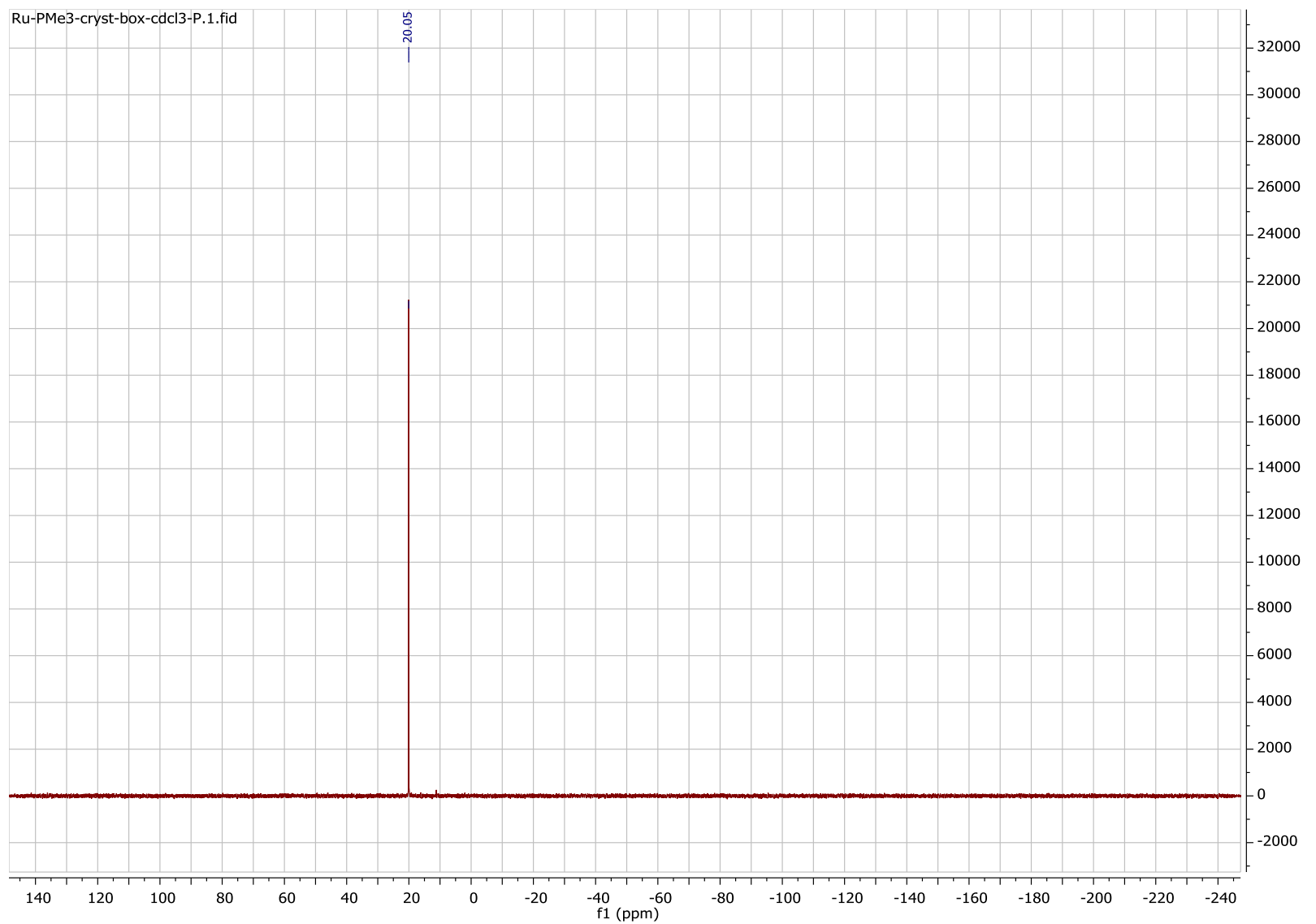




\section{$\left[\mathrm{Ru}_{2}\left(\mathrm{PEt}_{3}\right)_{6} \mathrm{Cl}_{3}\right][\mathrm{Cl}]$}

\section{${ }^{1} \mathbf{H}$}

JM1072A_1H.1.fid

AV-500 TBI probe

$1 \mathrm{H} 1 \mathrm{D}$ NMR

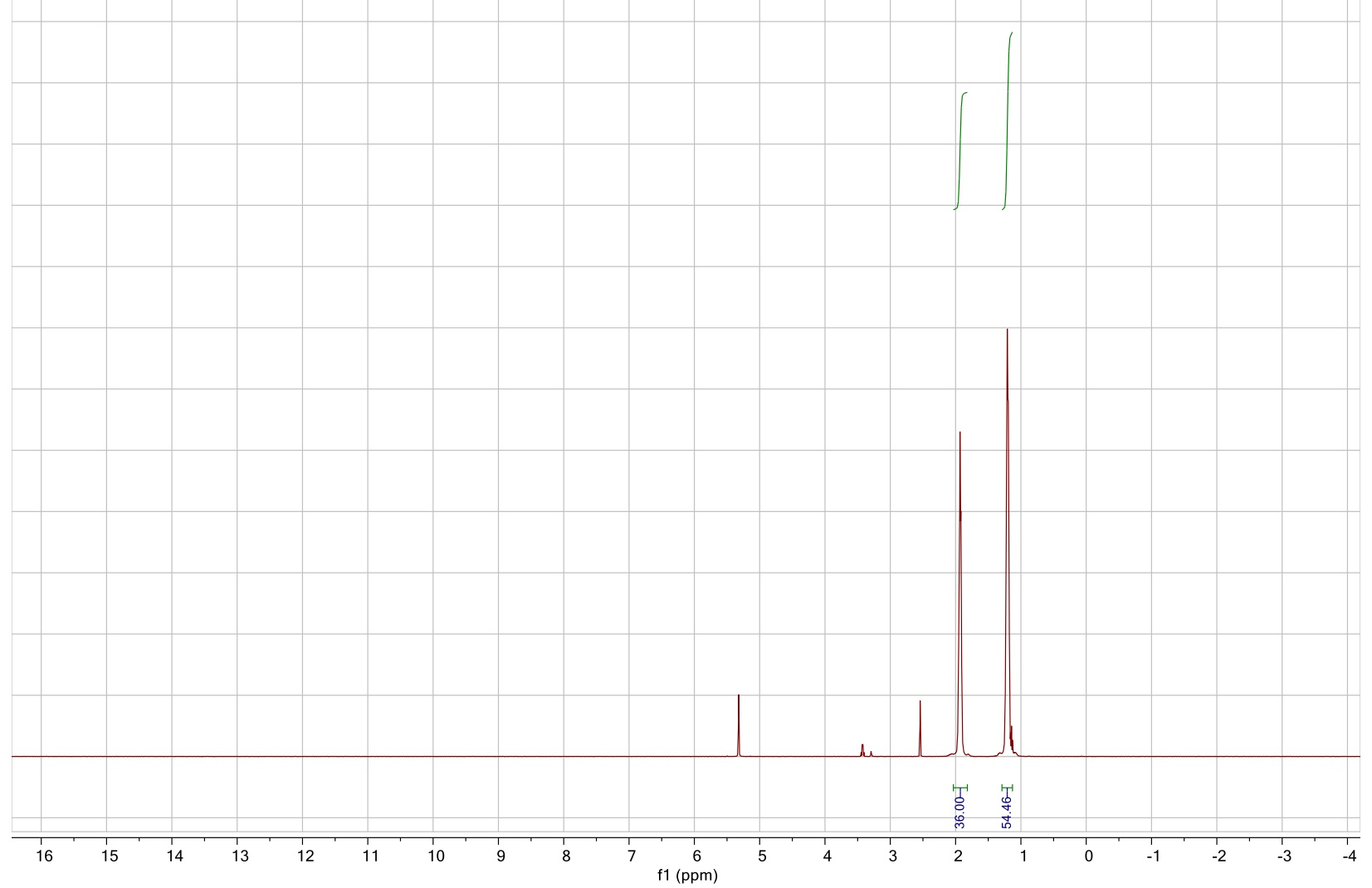


${ }^{31} \mathbf{P}$

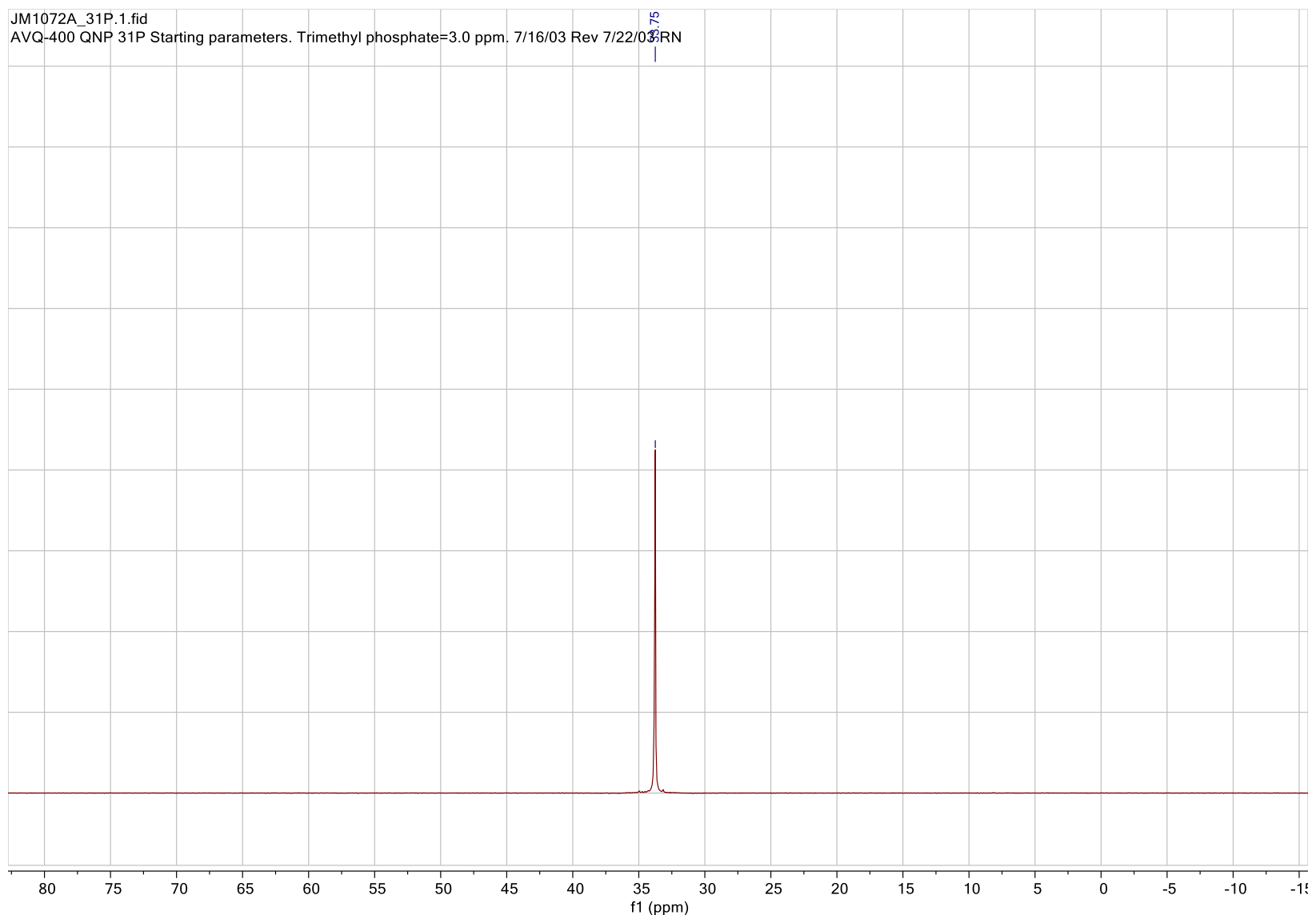


$\left[\mathrm{Ru}\left(\mathrm{P}^{\mathrm{n}} \mathrm{Pr}_{3}\right)_{6} \mathrm{Cl}_{3}\right][\mathrm{Cl}]$

${ }^{1} \mathbf{H}$

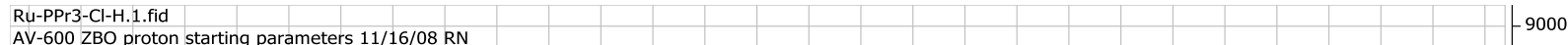

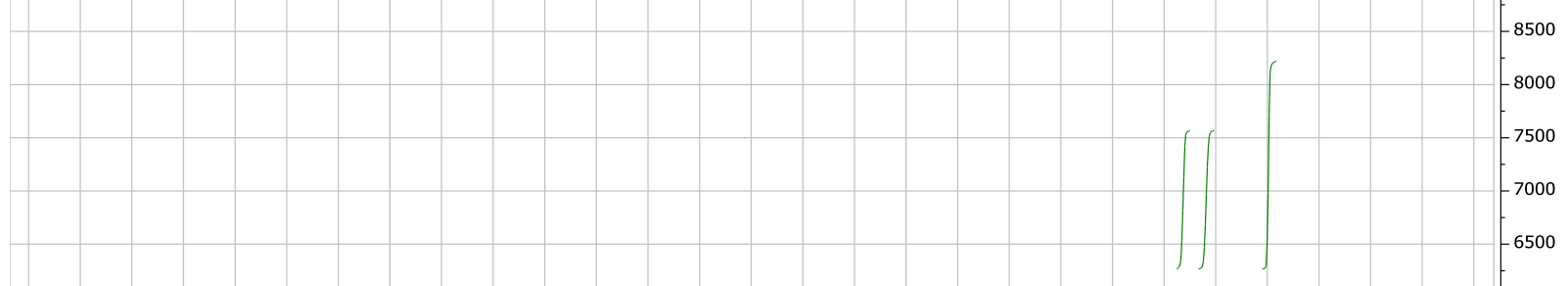

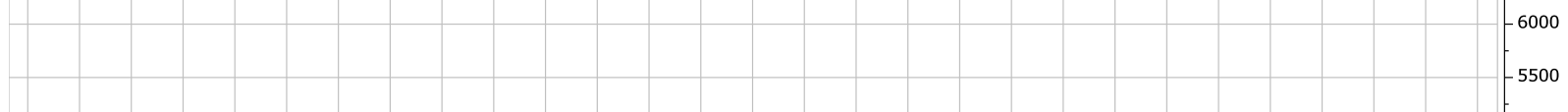

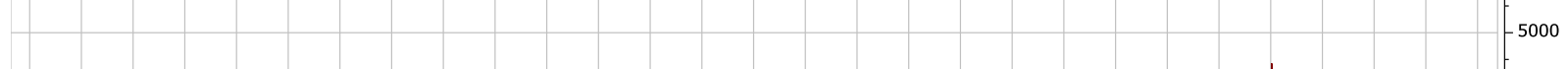
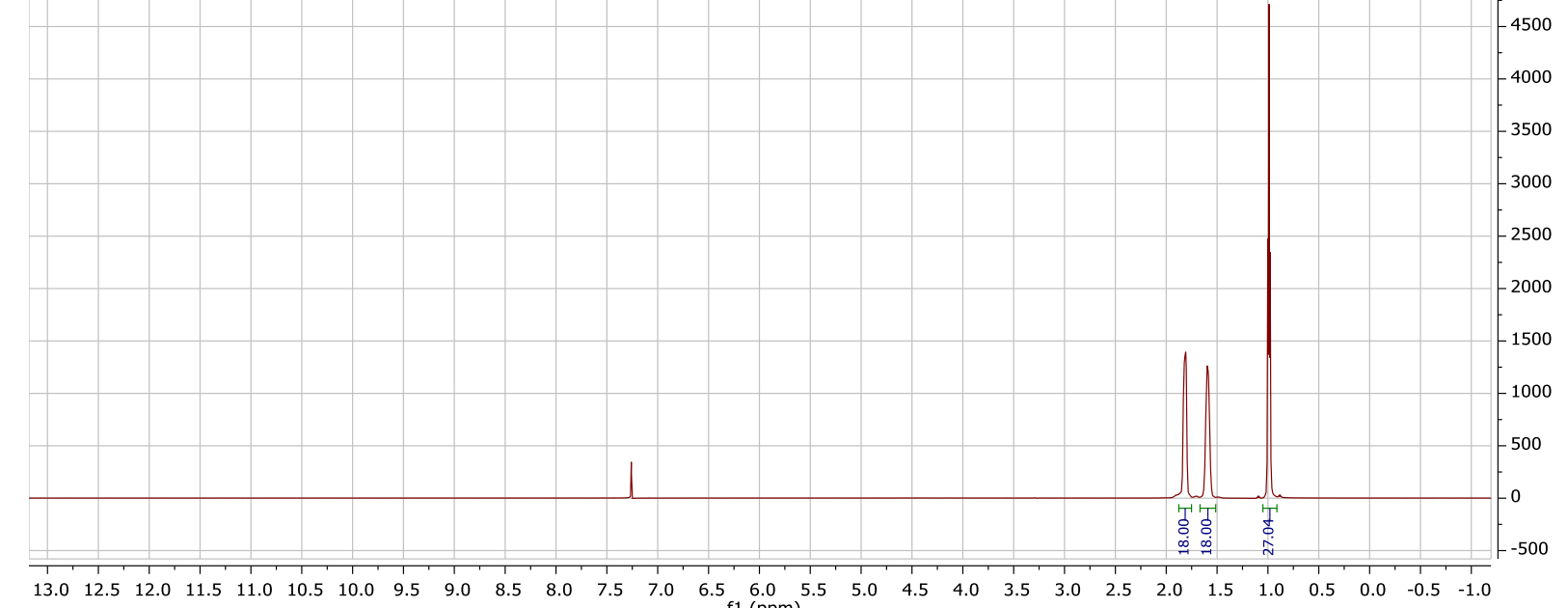


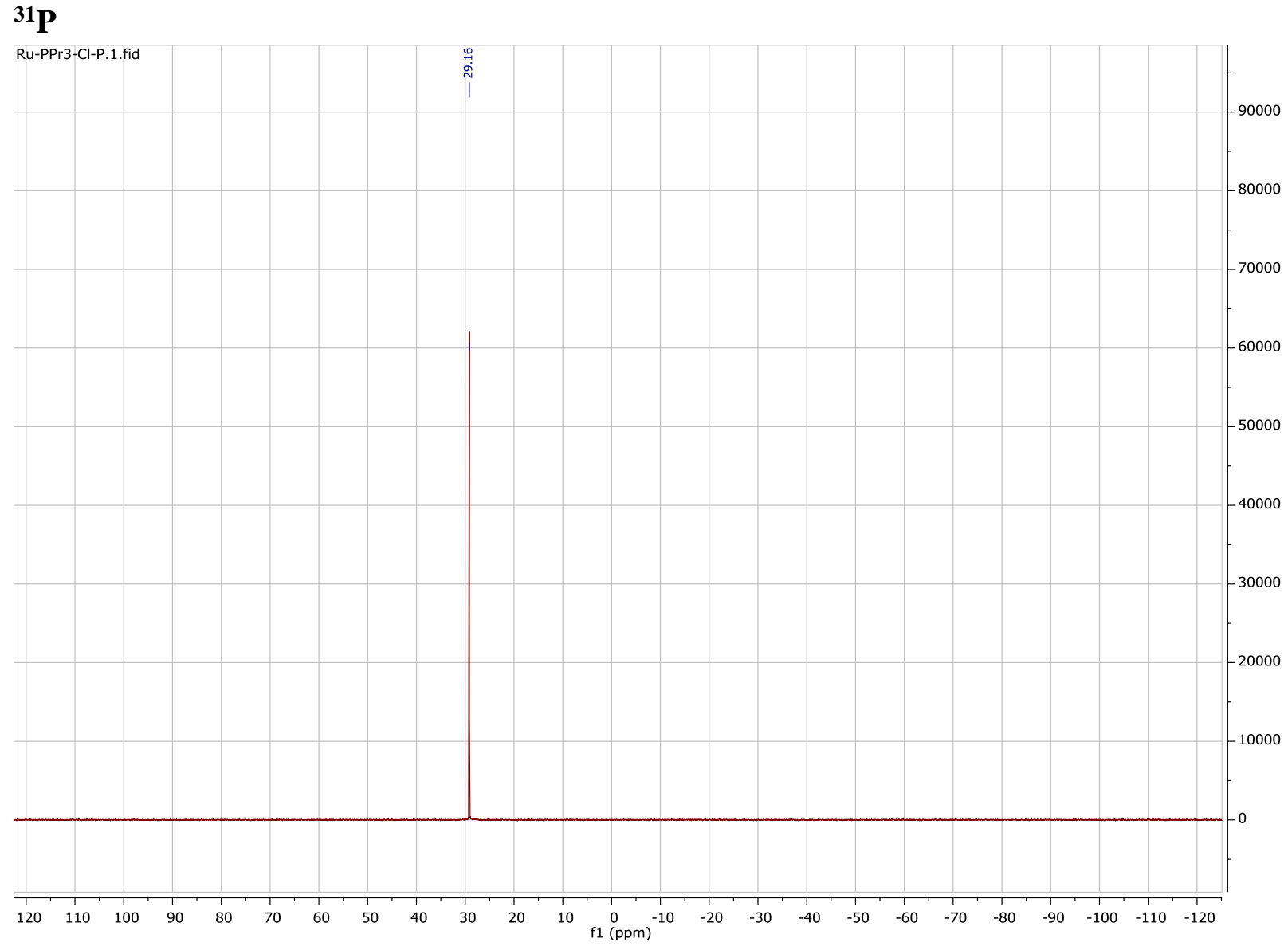




\section{$\left[\mathrm{Ru}_{2}\left(\mathrm{PMePh}_{2}\right)_{6} \mathrm{Cl}_{3}\right][\mathrm{Cl}]$}

\section{${ }^{1} \mathbf{H}$}

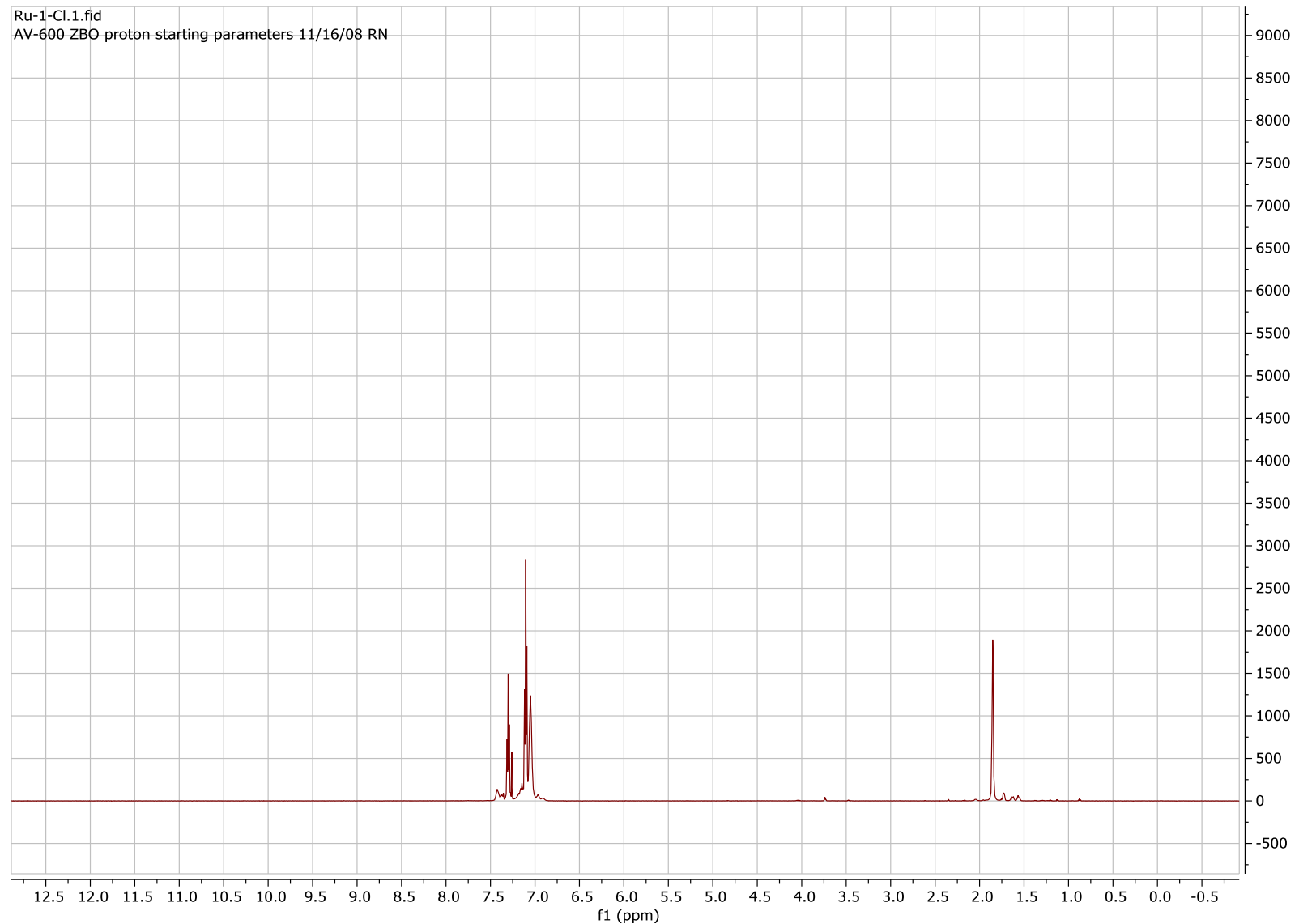




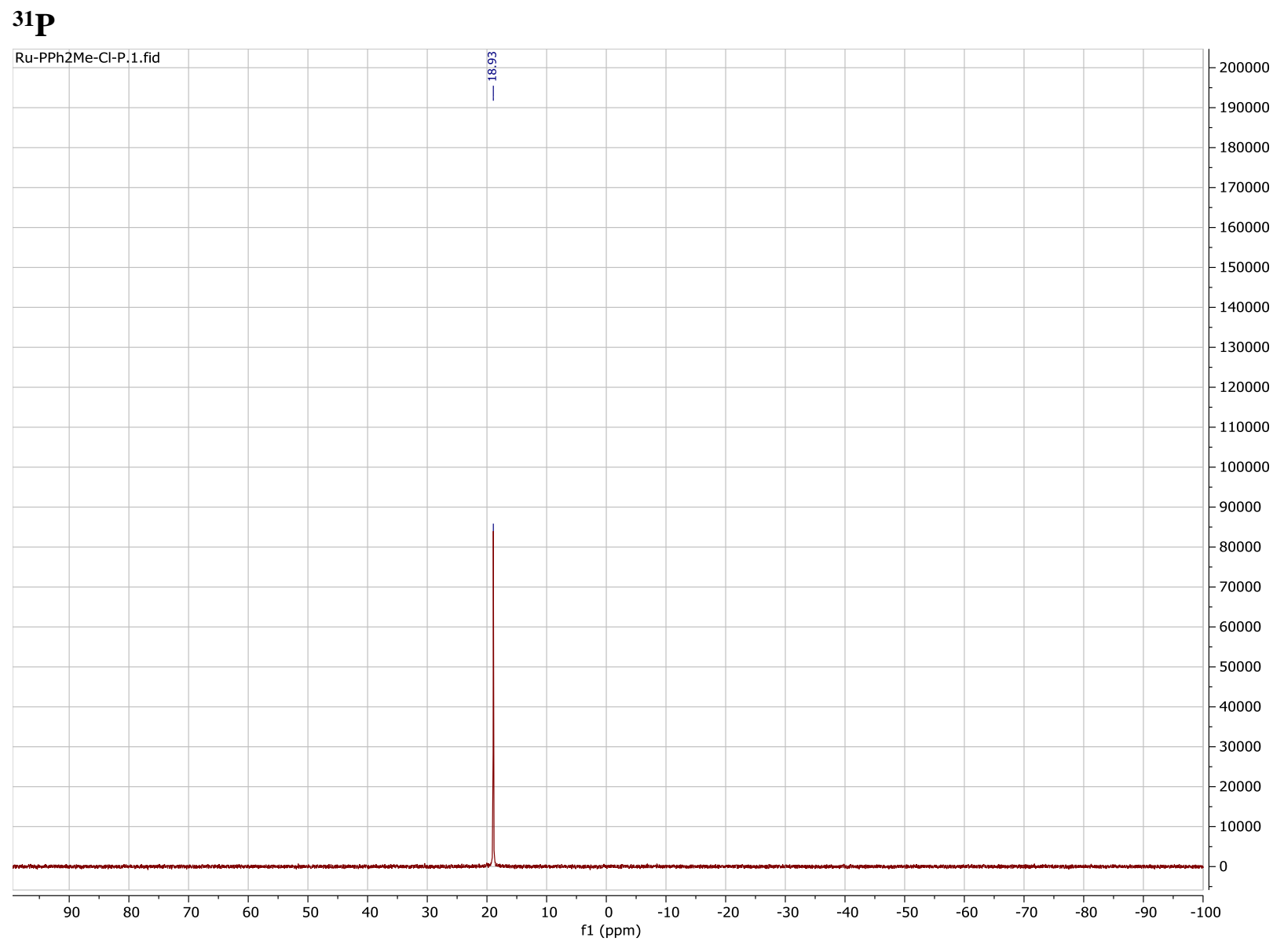


$c i s-\operatorname{Ru}\left(\mathrm{Et}_{2} \mathrm{P}\left(\mathrm{CH}_{2}\right)_{4} \mathrm{PEt}_{2}\right)_{2} \mathrm{Cl}_{2}$

${ }^{1} \mathbf{H}$

\begin{tabular}{l}
$\begin{array}{l}\text { Ru-Et2P-butyl-PEt2-Cl-H.1.fid } \\
\text { AV-600 ZBO proton starting parameters 11/16/08 RN }\end{array}$ \\
\hline
\end{tabular}

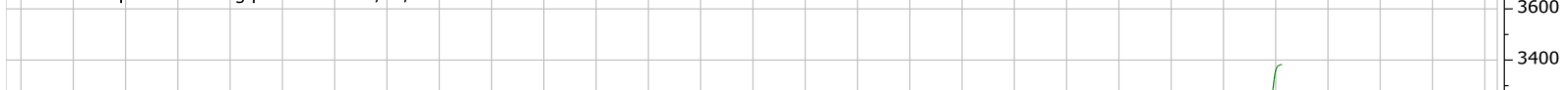

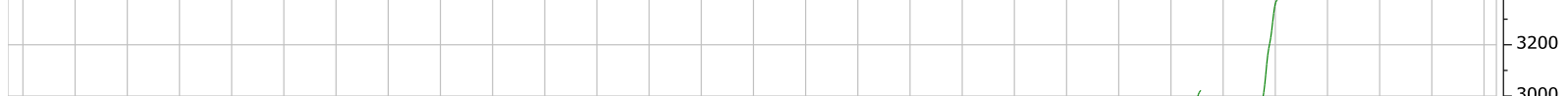

\begin{tabular}{|l|l|l|l|l|l|l|l|l|l|l|l|l|l|l|l|l|l|l|l|}
\hline & & & & \\
\hline
\end{tabular}
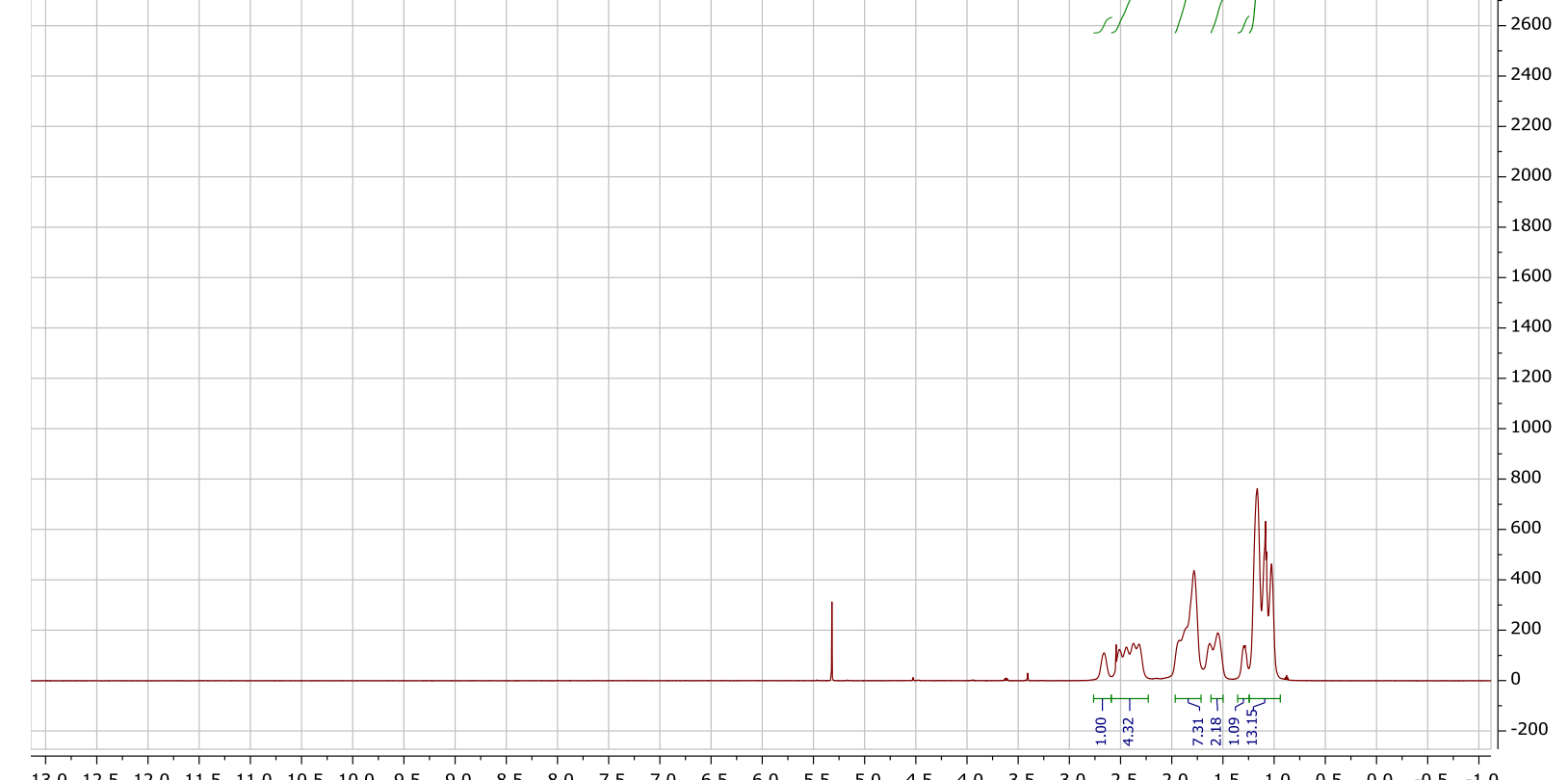

$\begin{array}{llllllllllllllllllllllllllllllllll}13.0 & 12.5 & 12.0 & 11.5 & 11.0 & 10.5 & 10.0 & 9.5 & 9.0 & 8.5 & 8.0 & 7.5 & 7.0 & 6.5 & 6.0 & 5.5 & 5.0 & 4.5 & 4.0 & 3.5 & 3.0 & 2.5 & 2.0 & 1.5 & 1.0 & 0.5 & 0.0 & -0.5 & -1.0\end{array}$ 


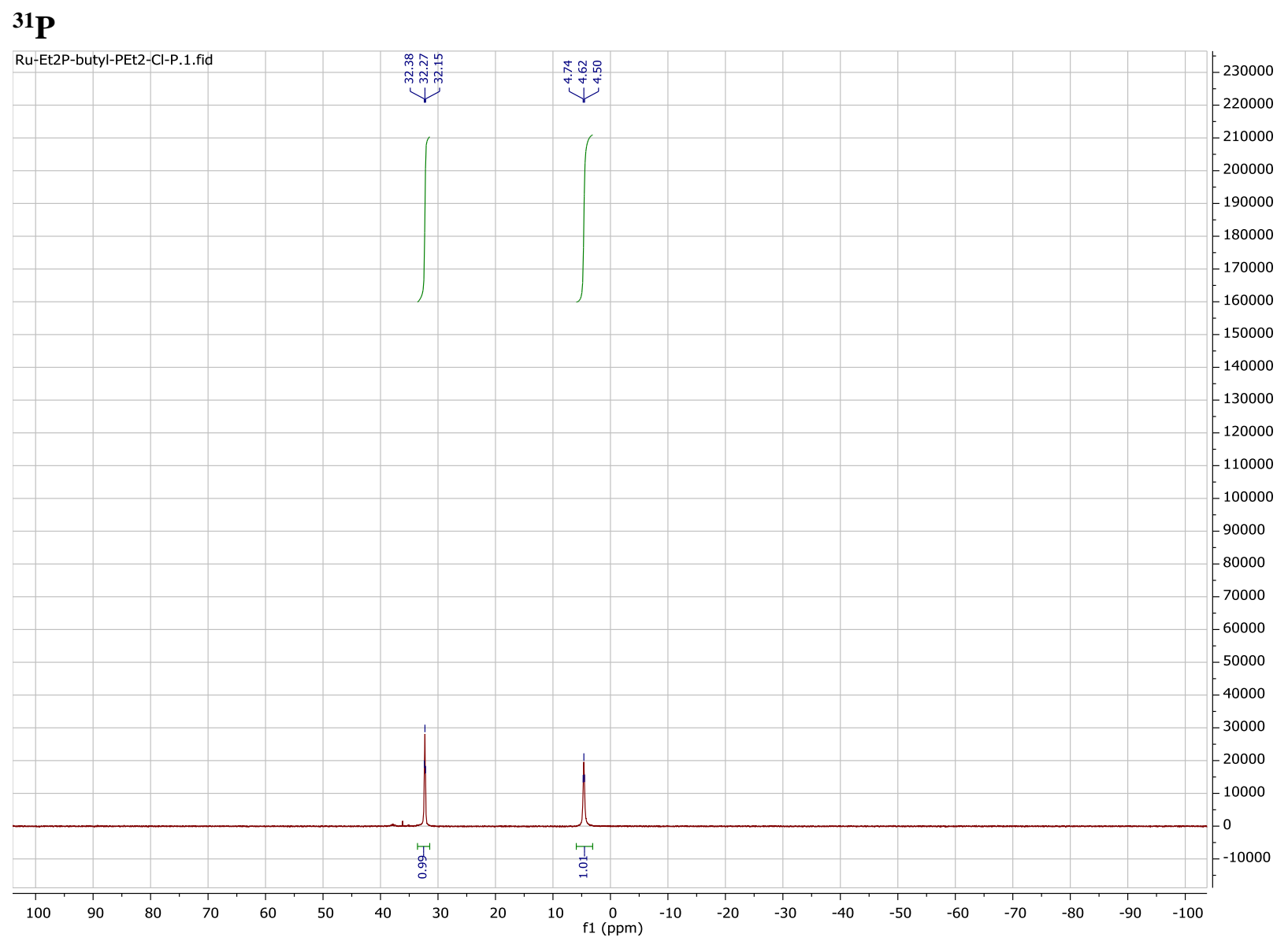

S63 


\section{$\left[\mathrm{Ru}_{2}\left(\mathbf{N}\left(\mathrm{CH}_{2} \mathrm{PEt}_{2}\right)_{3}\right)_{2} \mathrm{Cl}_{3}\right][\mathrm{Cl}]$}

${ }^{1} \mathbf{H}$

Ru-NCH2PEt23-Cl.1.fid

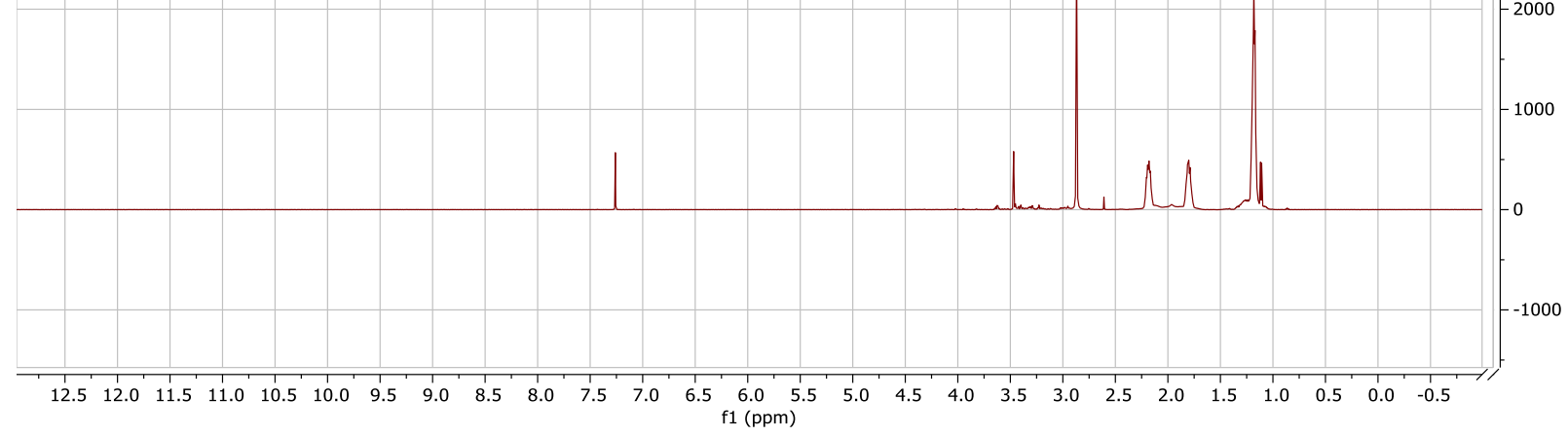




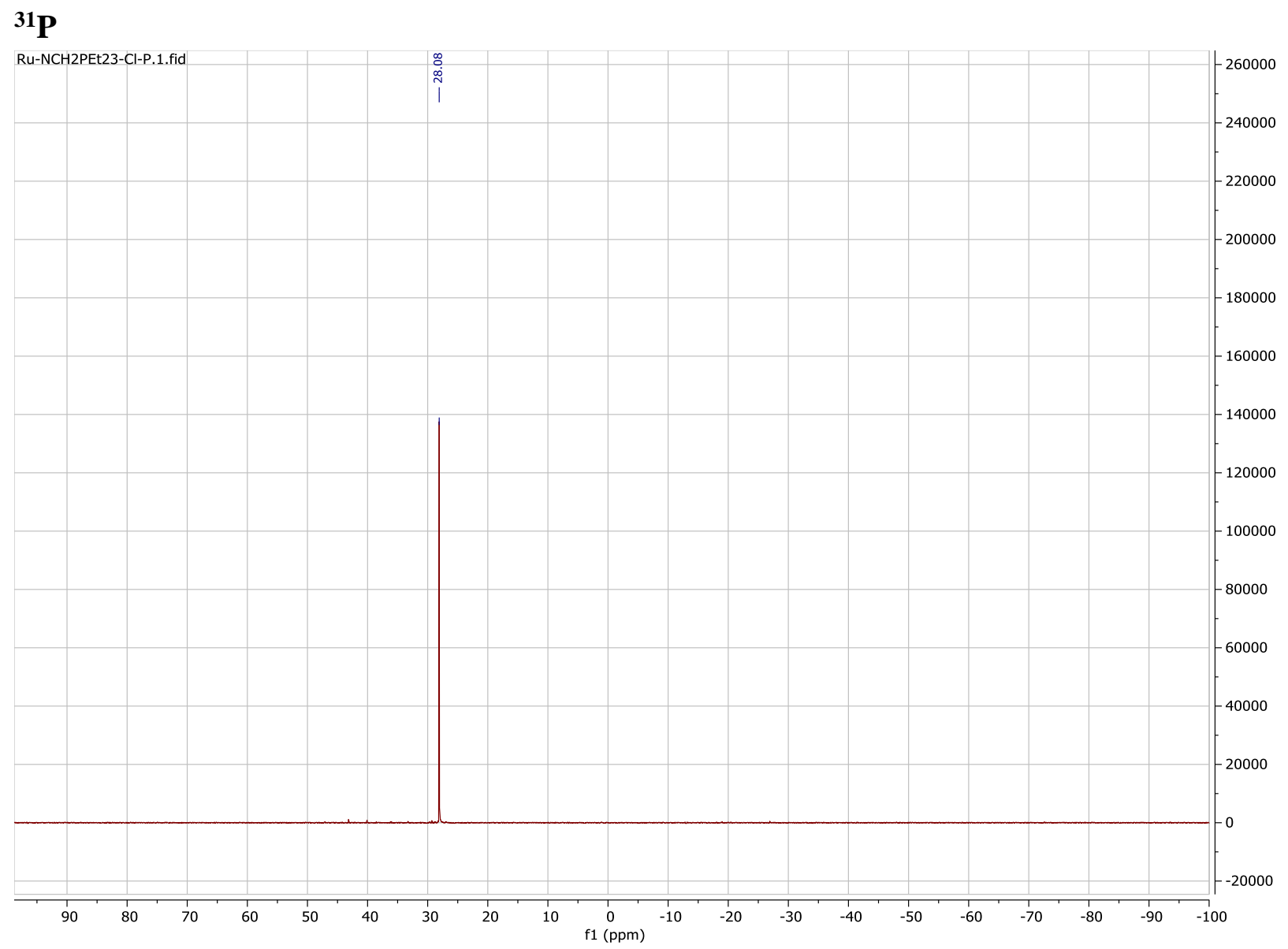




\section{$\operatorname{Ru}_{\left(\text {PEt}_{3}\right)_{3}\left(\text { NTf }_{2}\right)_{2}(\text { Ru-1) }}$}

${ }^{1} \mathbf{H}$

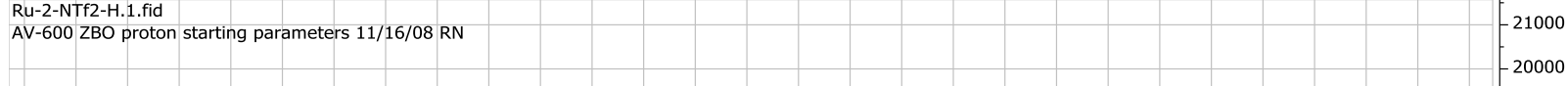

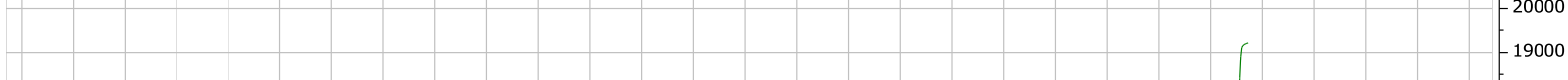

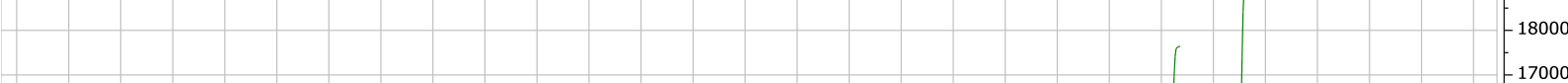

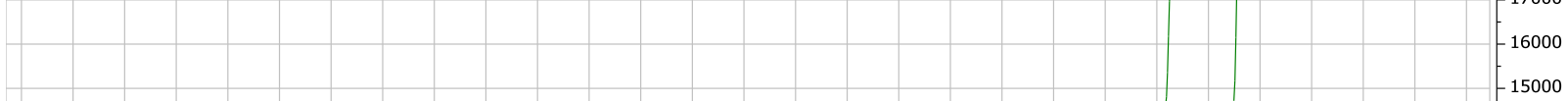

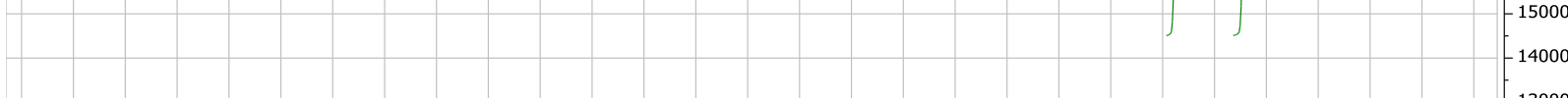
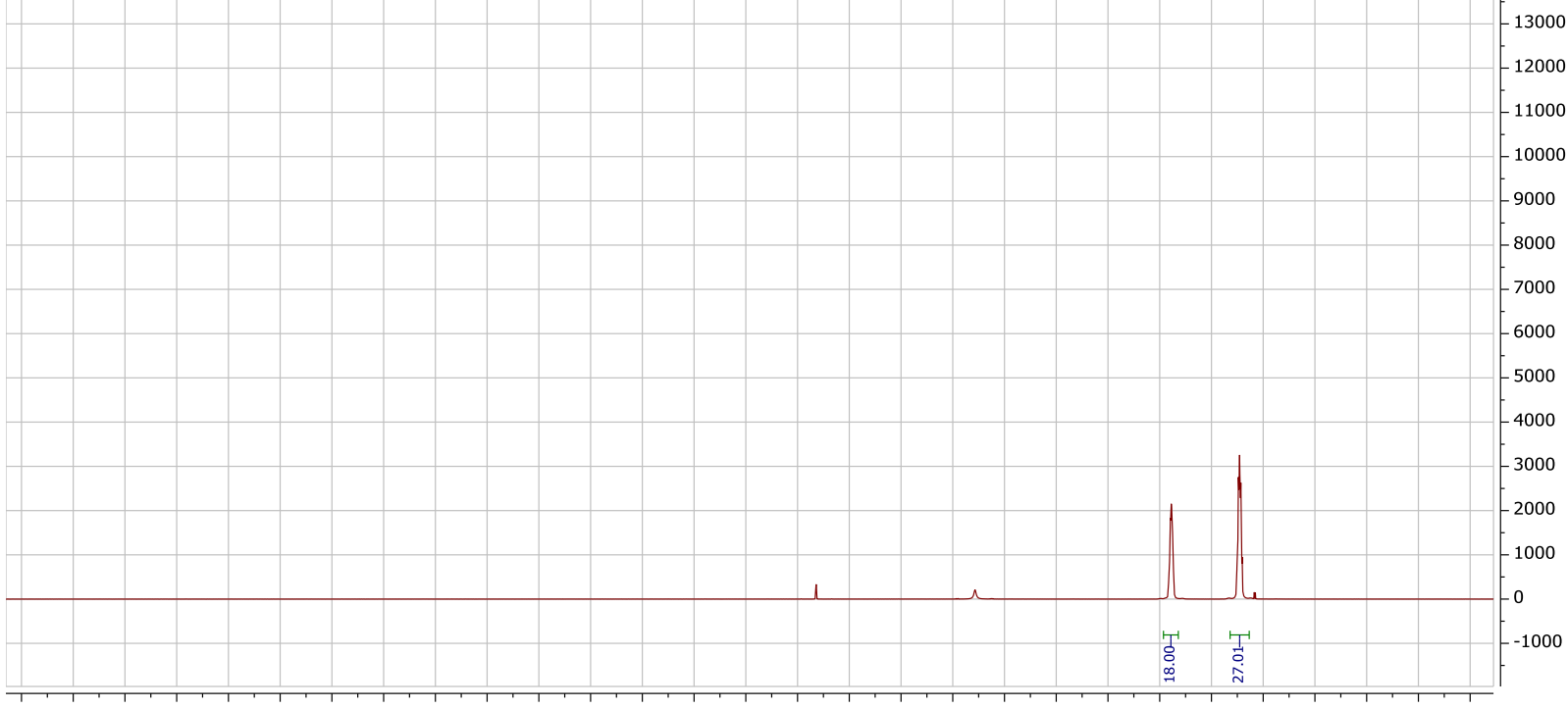

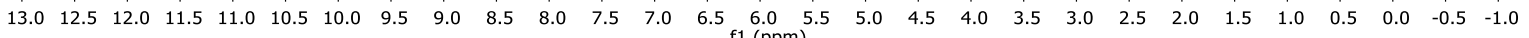
f1 (ppm) 
$31 \mathbf{P}$

JM1064A_31P.1.fid

AVQ-400 QNP 31P Starting parameters. Trimethyl phosphate $=3.0$ ppm. $7 \stackrel{\infty}{\mathrm{L}} 6 / 03 \mathrm{Rev} 7 / 22 / 03 \mathrm{RN}$
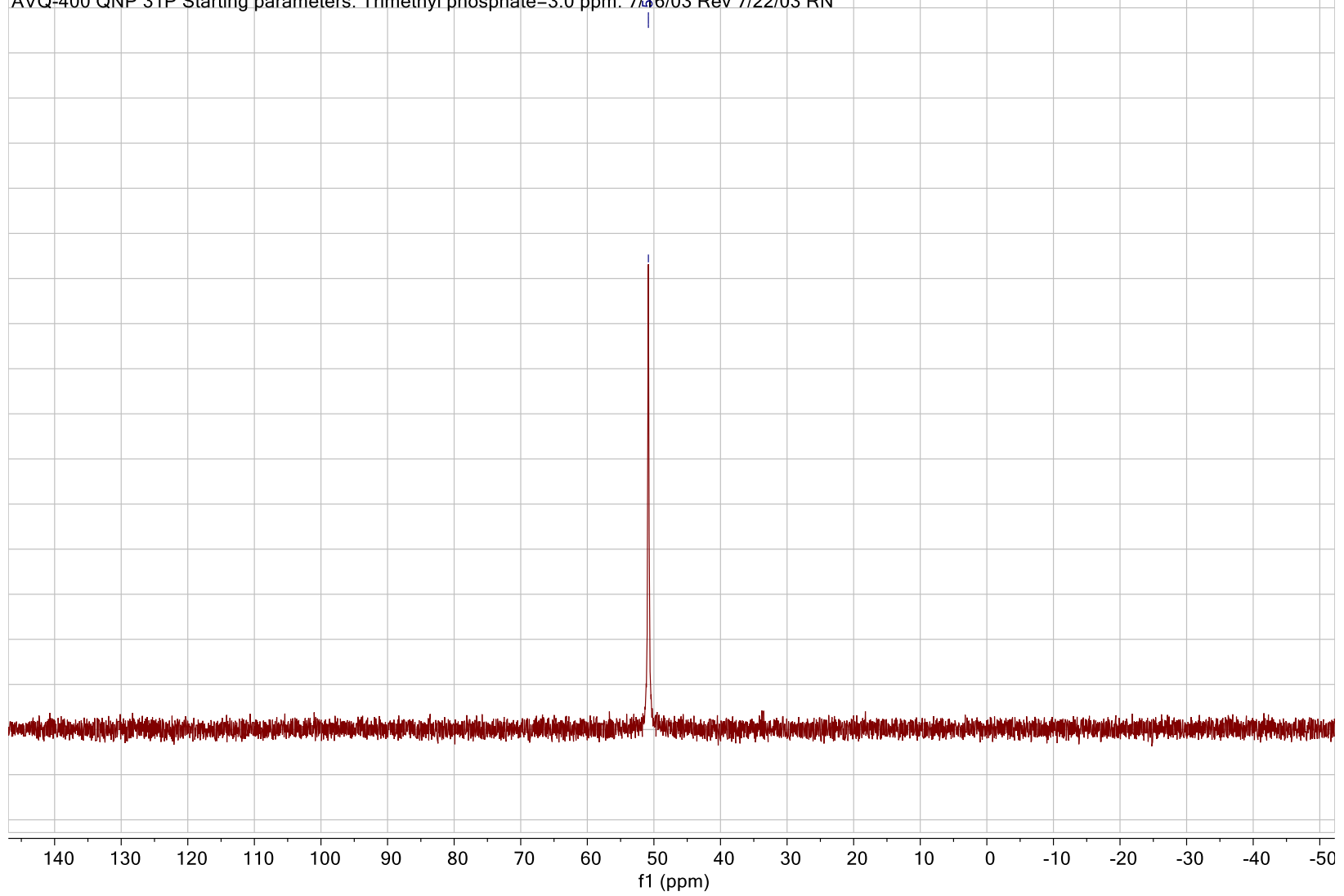


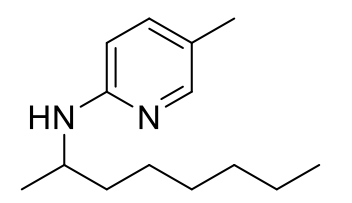

\section{5-methyl- $N$-(octan-2-yl)pyridine-2-amine (2a)}

${ }^{1} \mathbf{H}$

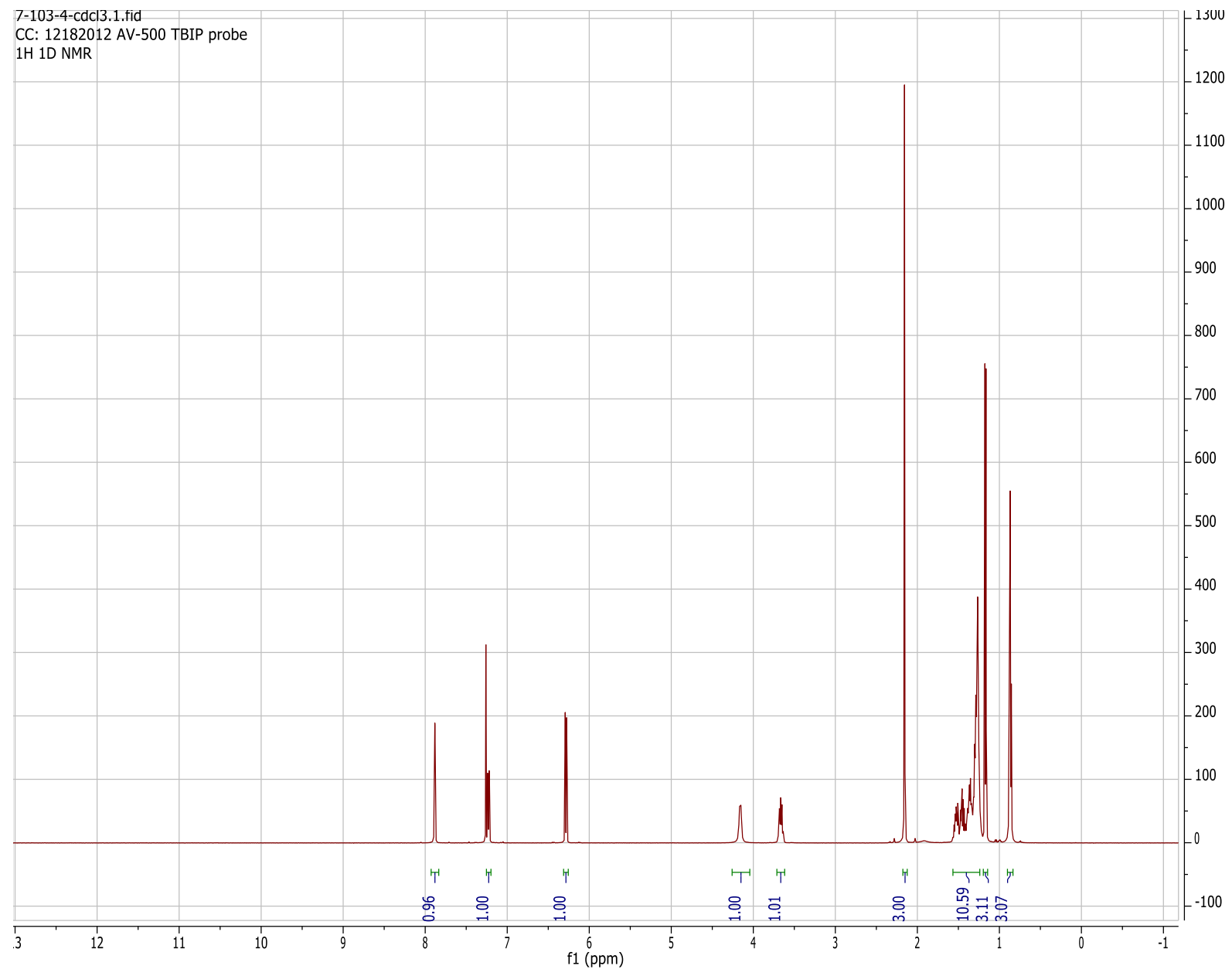


${ }^{13} \mathrm{C}$

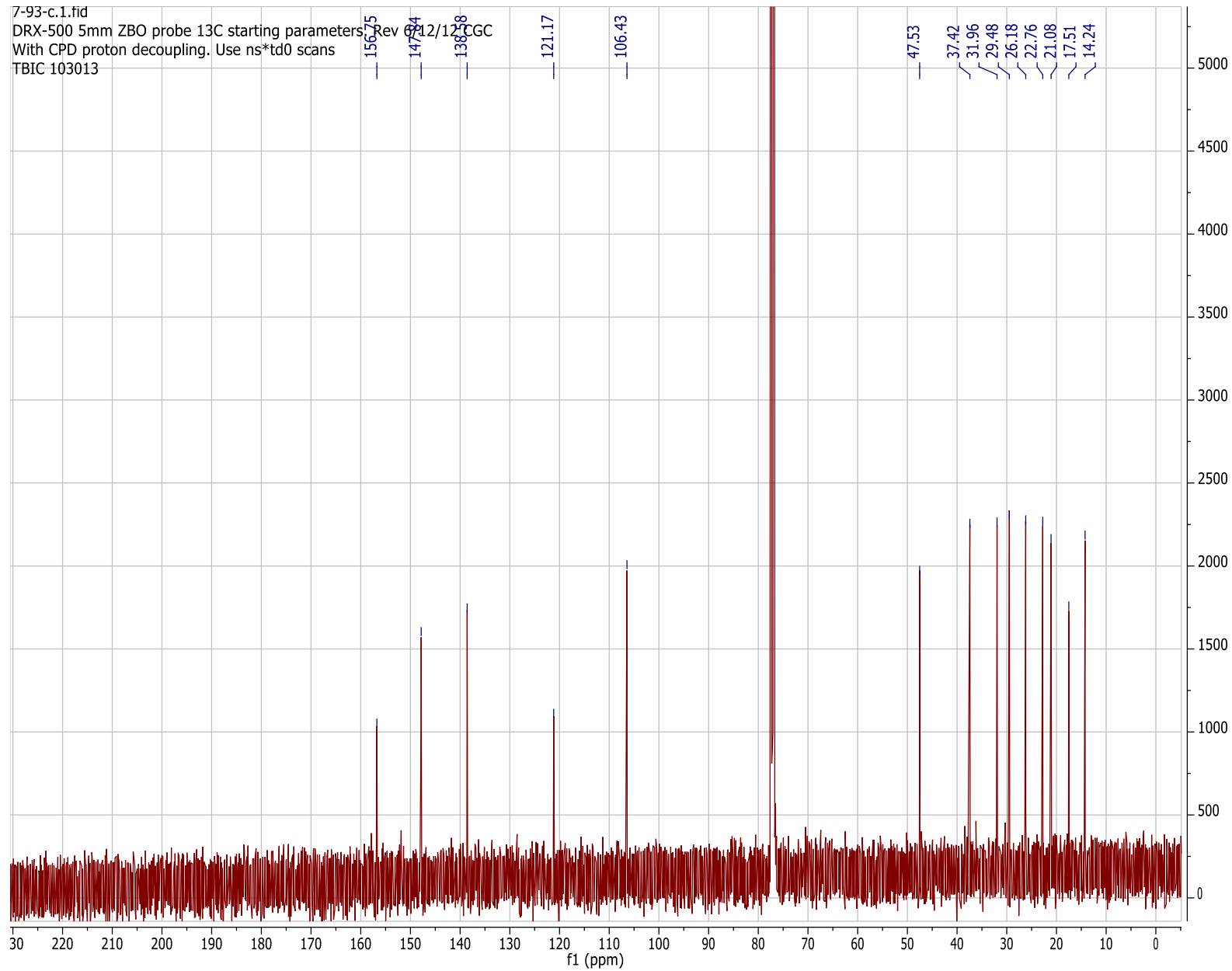


(1)

5-methyl-N-(dodecan-2-yl)pyridine-2-amine (3a)

${ }^{1} \mathbf{H}$

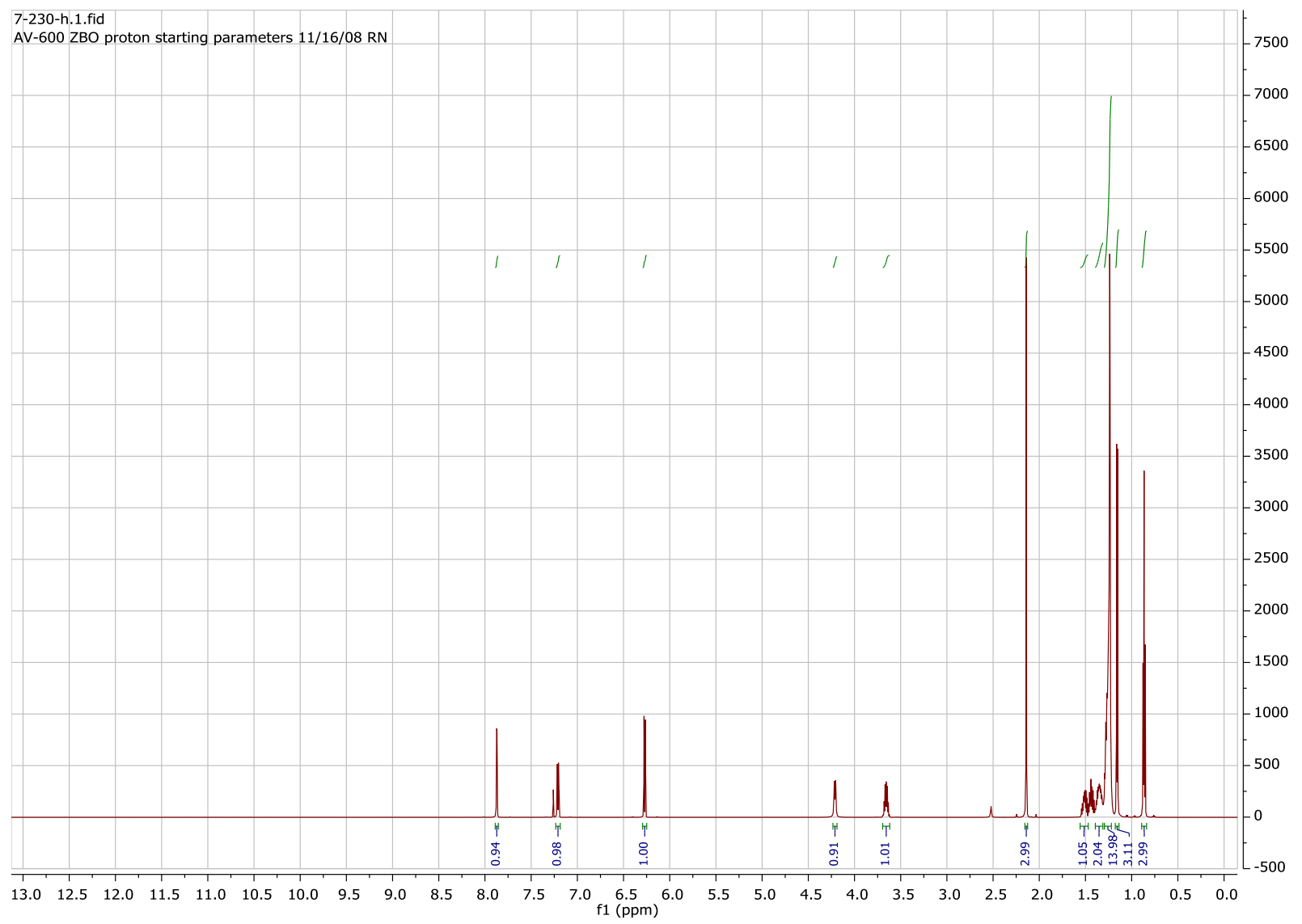


${ }^{13} \mathrm{C}$

7-230-c.1.fid

7-230-c.1.fid

$A Q \_M O D=D Q D$

-600 ZBO carbon starting paramet

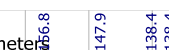

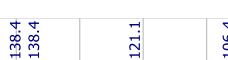

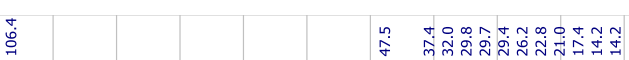

\begin{tabular}{|l|l|l|l|l|l|l|l|l|l|l|l|}
\hline-10000 & \\
\hline-9000
\end{tabular}

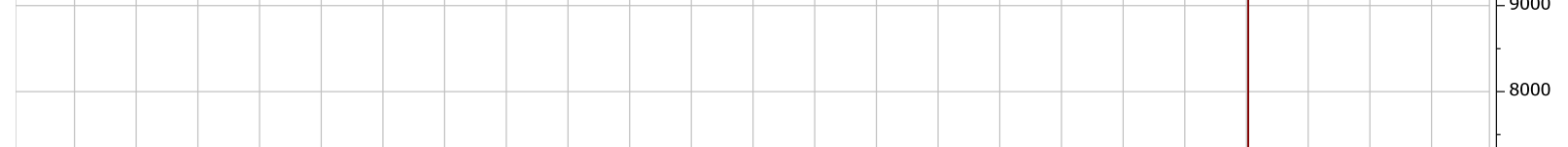
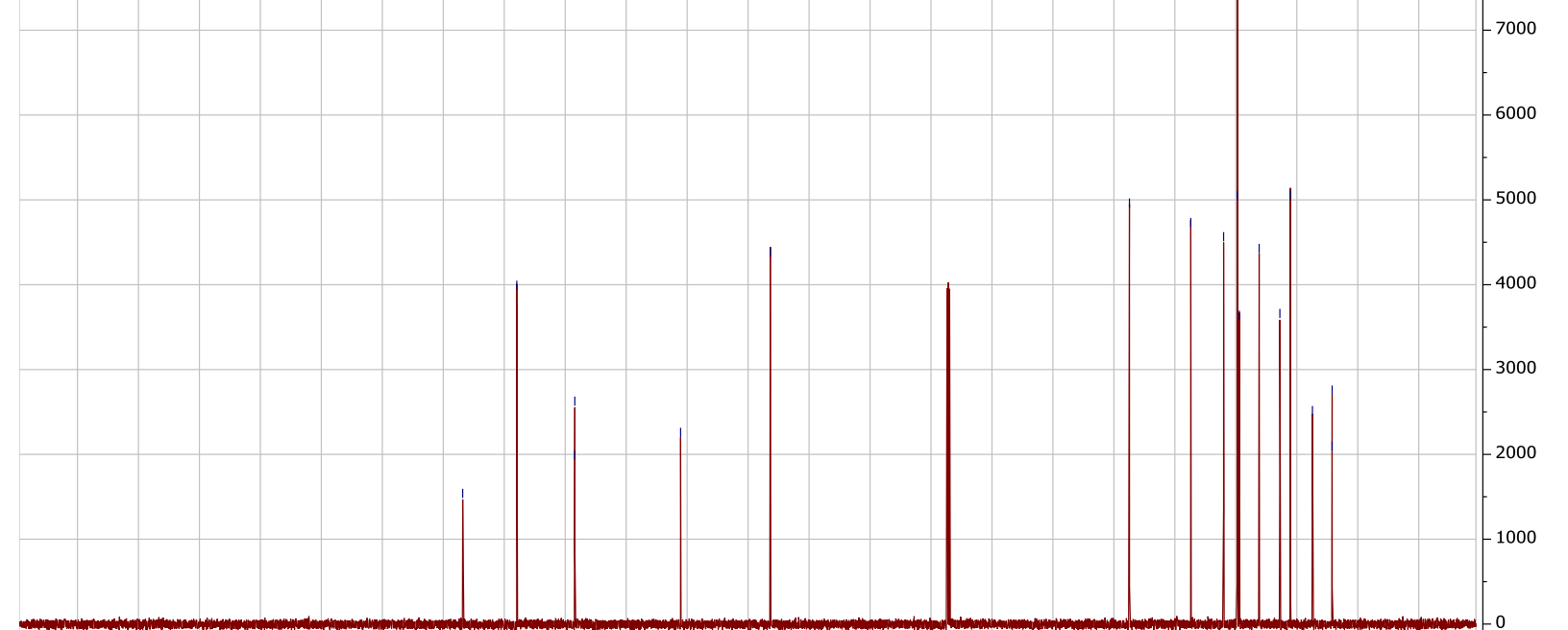

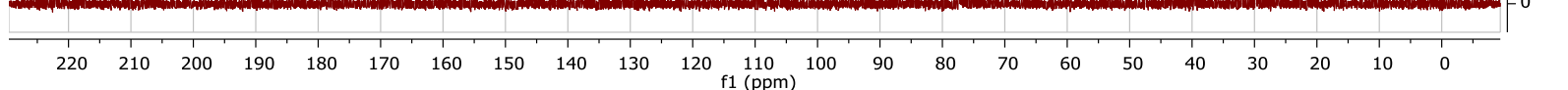


$\overbrace{}^{2}$

5-methyl- $N$-(1-phenylpropan-2-yl)pyridine-2-amine (4a)

${ }^{1} \mathbf{H}$

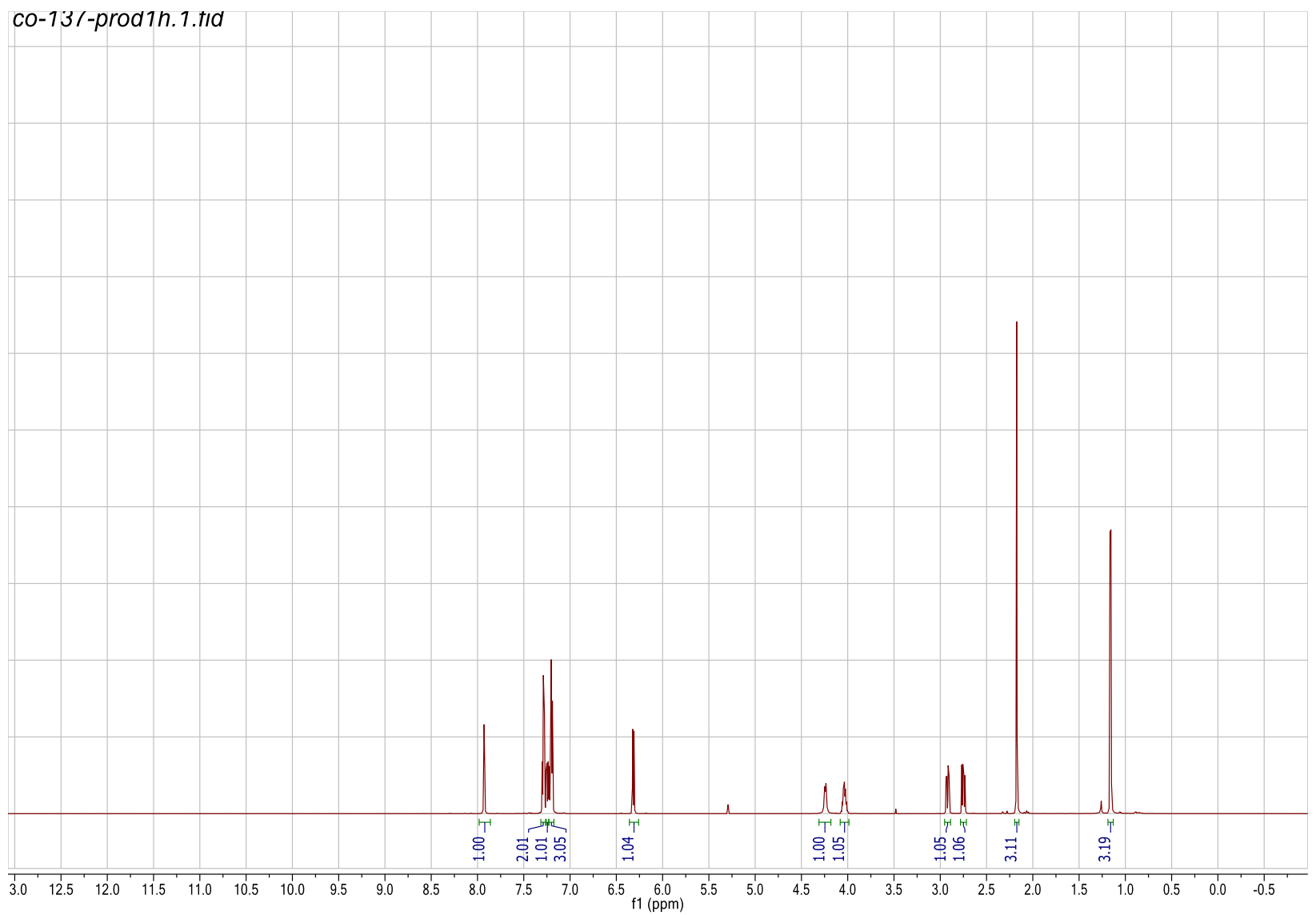


${ }^{13} \mathrm{C}$

CU-13/-prodtınc13.1.tıd
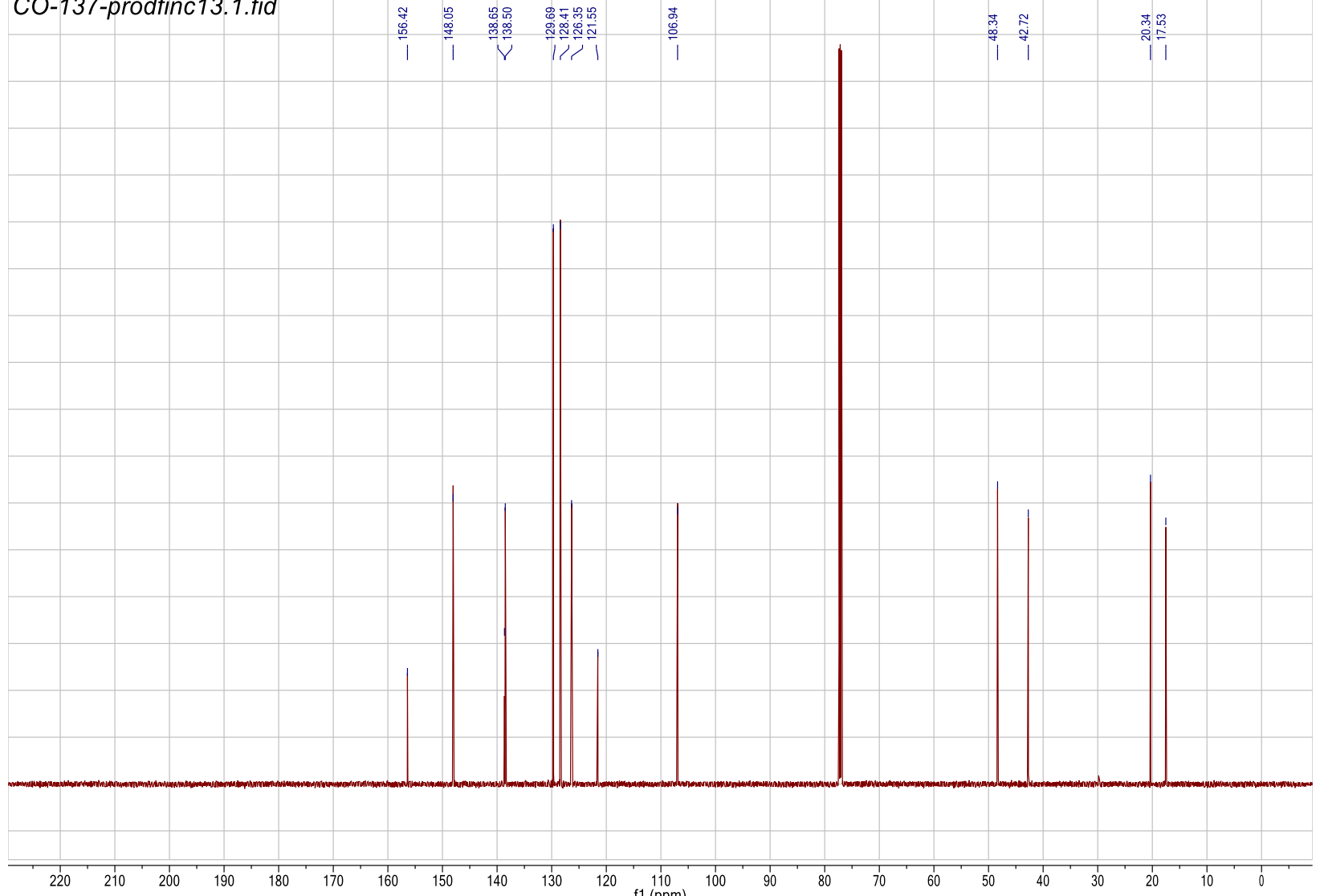
<smiles>COc1ccc(CC(C)Nc2ccc(C)cn2)cc1OC</smiles>

N-(1-(3,4-dimethoxyphenyl)propan-2-yl)-5-methylpyridin-2-amine (5a)

${ }^{1} \mathbf{H}$

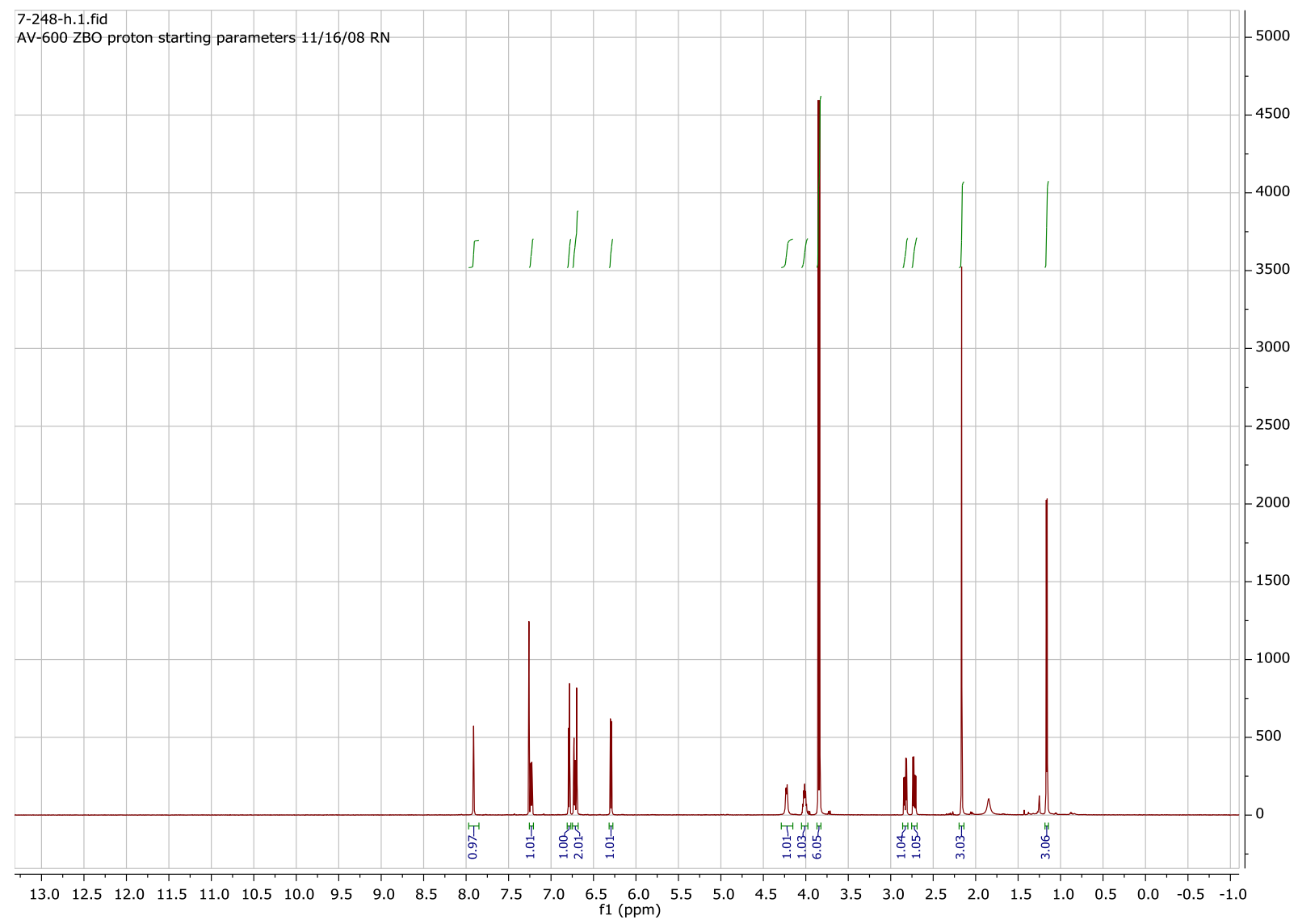


${ }^{13} \mathrm{C}$

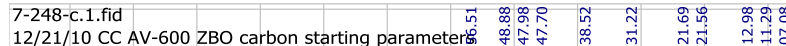

AQ $M O D=C A D$
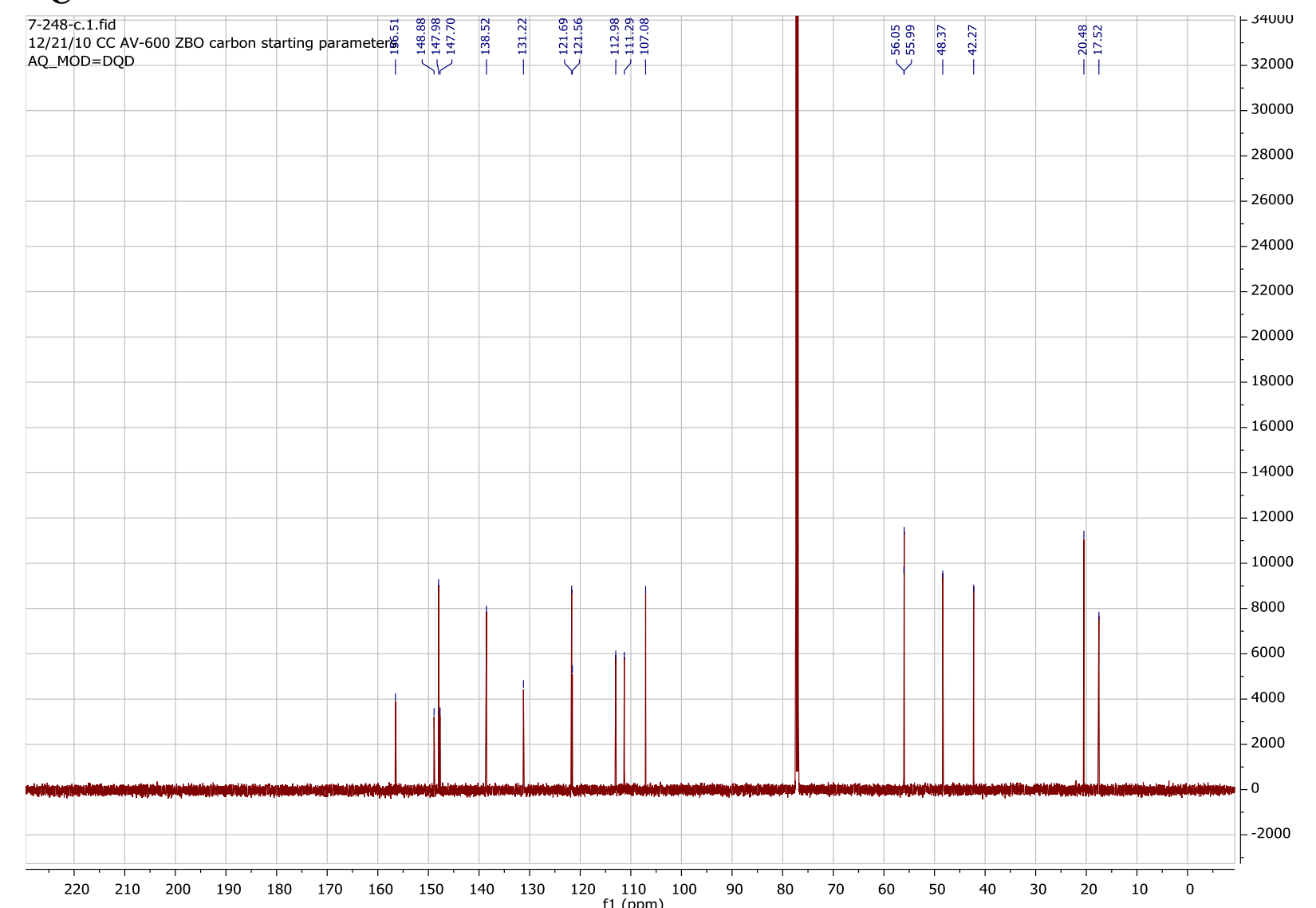
${ }_{1}$

5-methyl- $N$-(1-(4-fluoro-phenyl)propan-2-yl)pyridine-2-amine (6a)

${ }^{1} \mathbf{H}$

co-148-prod1h.1.tıd

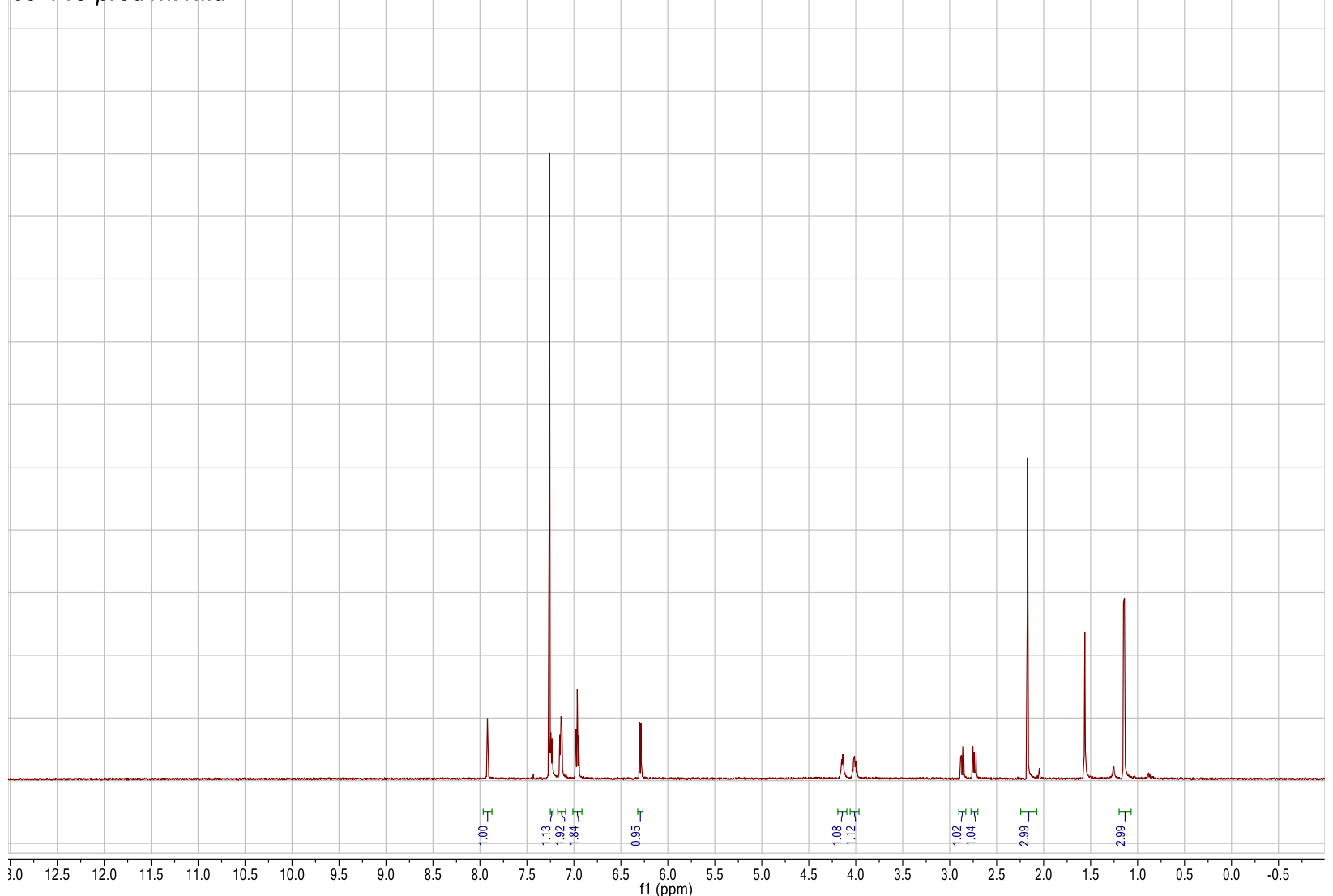


${ }^{13} \mathrm{C}$

co-148-prod13c.1.tid

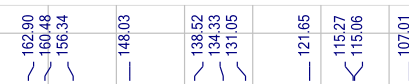
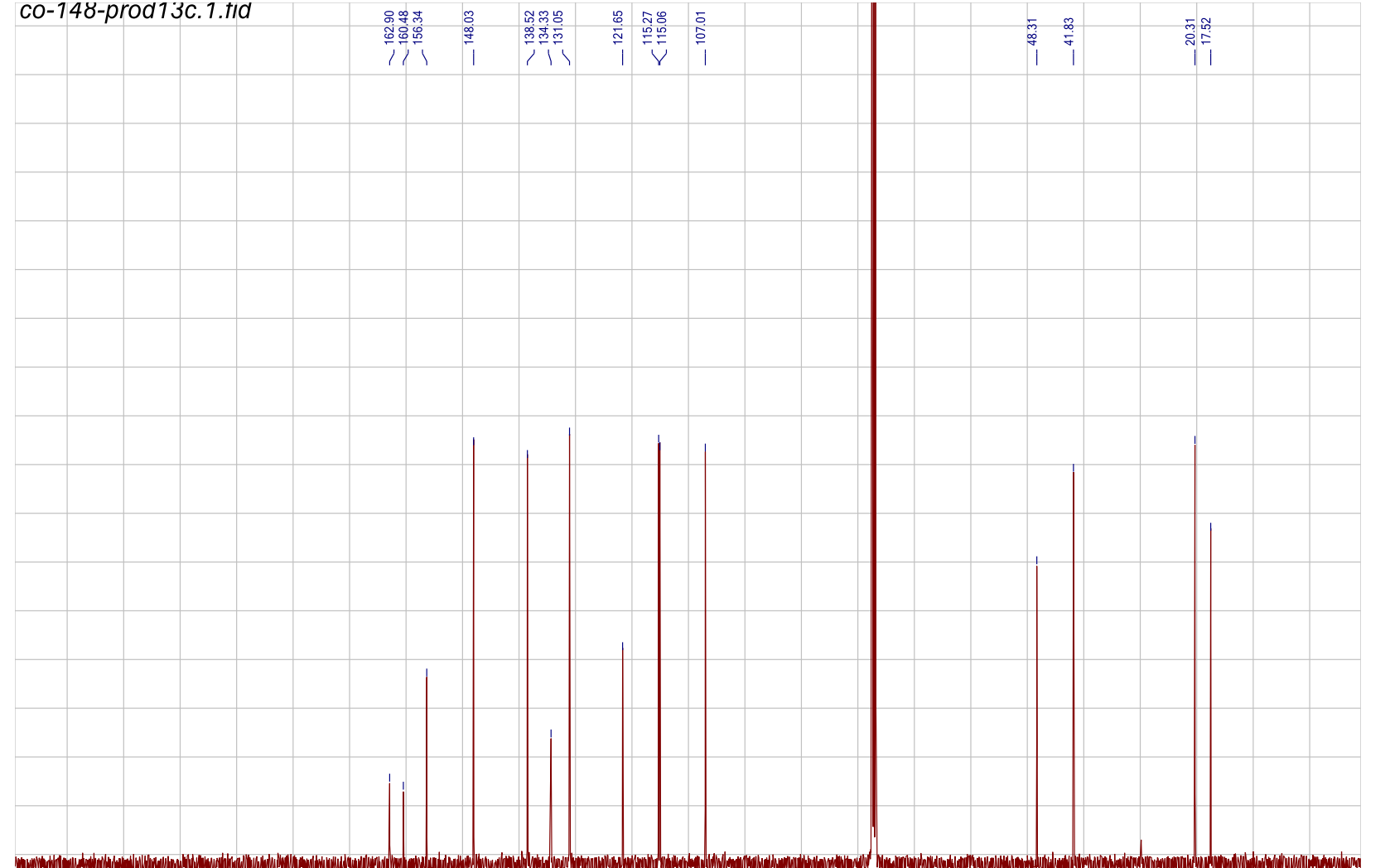

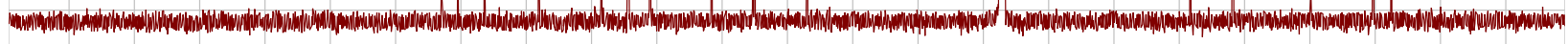

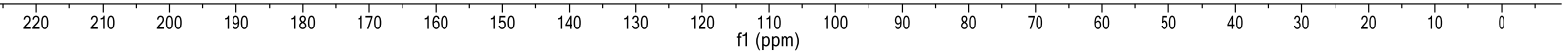


<smiles>CCNC(=O)CCCCCCCC(C)Nc1ccc(C)cn1</smiles>

N,N-diethyl-9-((5-methylpyridin-2-yl)amino)decanamide (7a)

${ }^{1} \mathbf{H}$

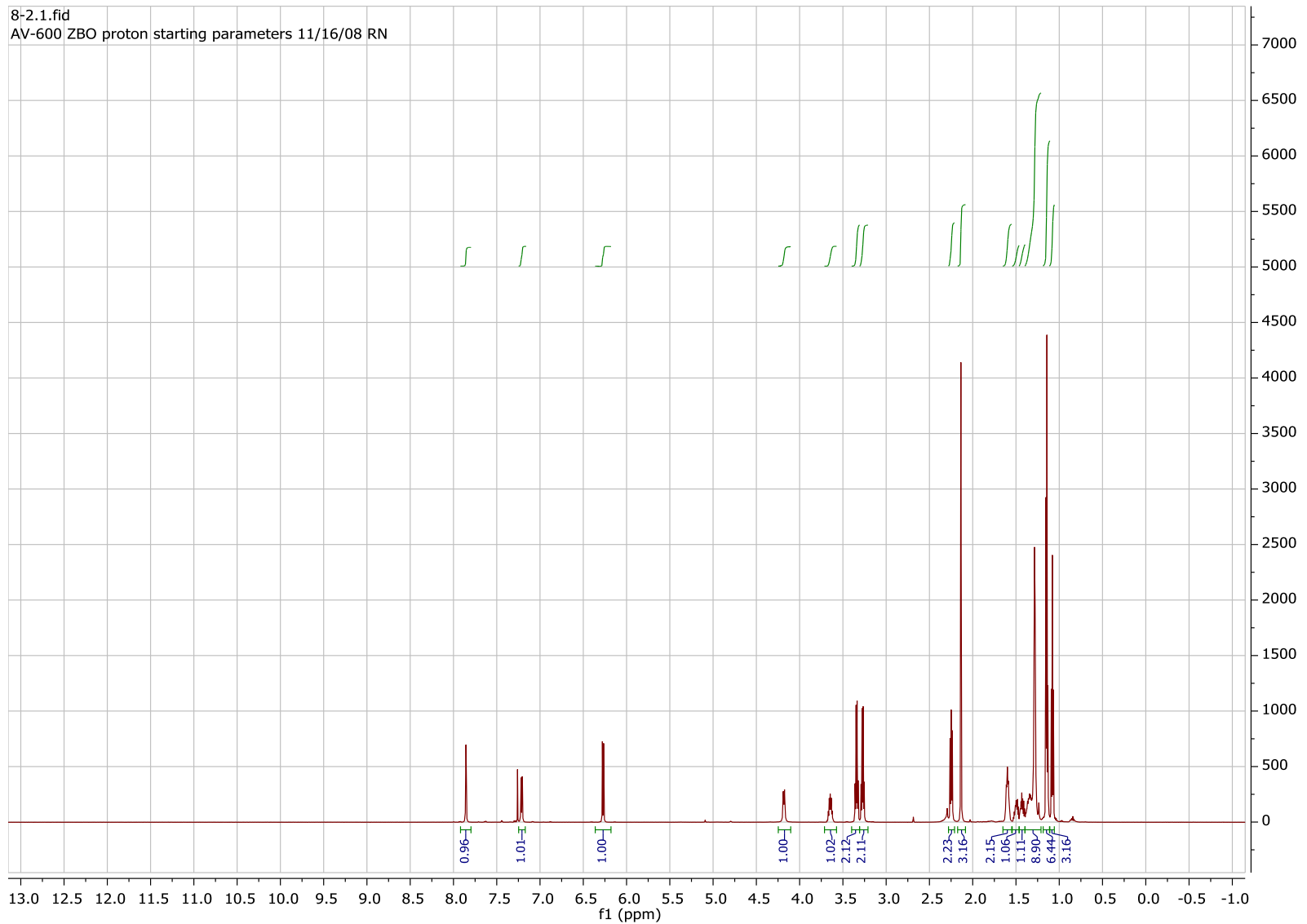


${ }^{13} \mathrm{C}$

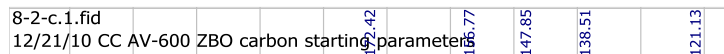

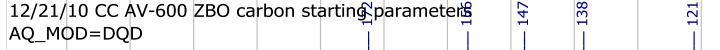

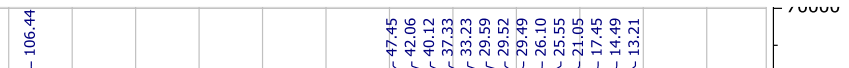

.

$\begin{array}{llll}1 & 1 & 1\end{array}$

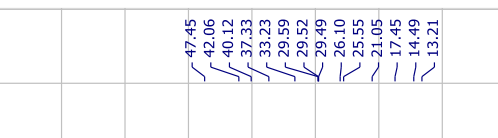

$-65000$

$-60000$
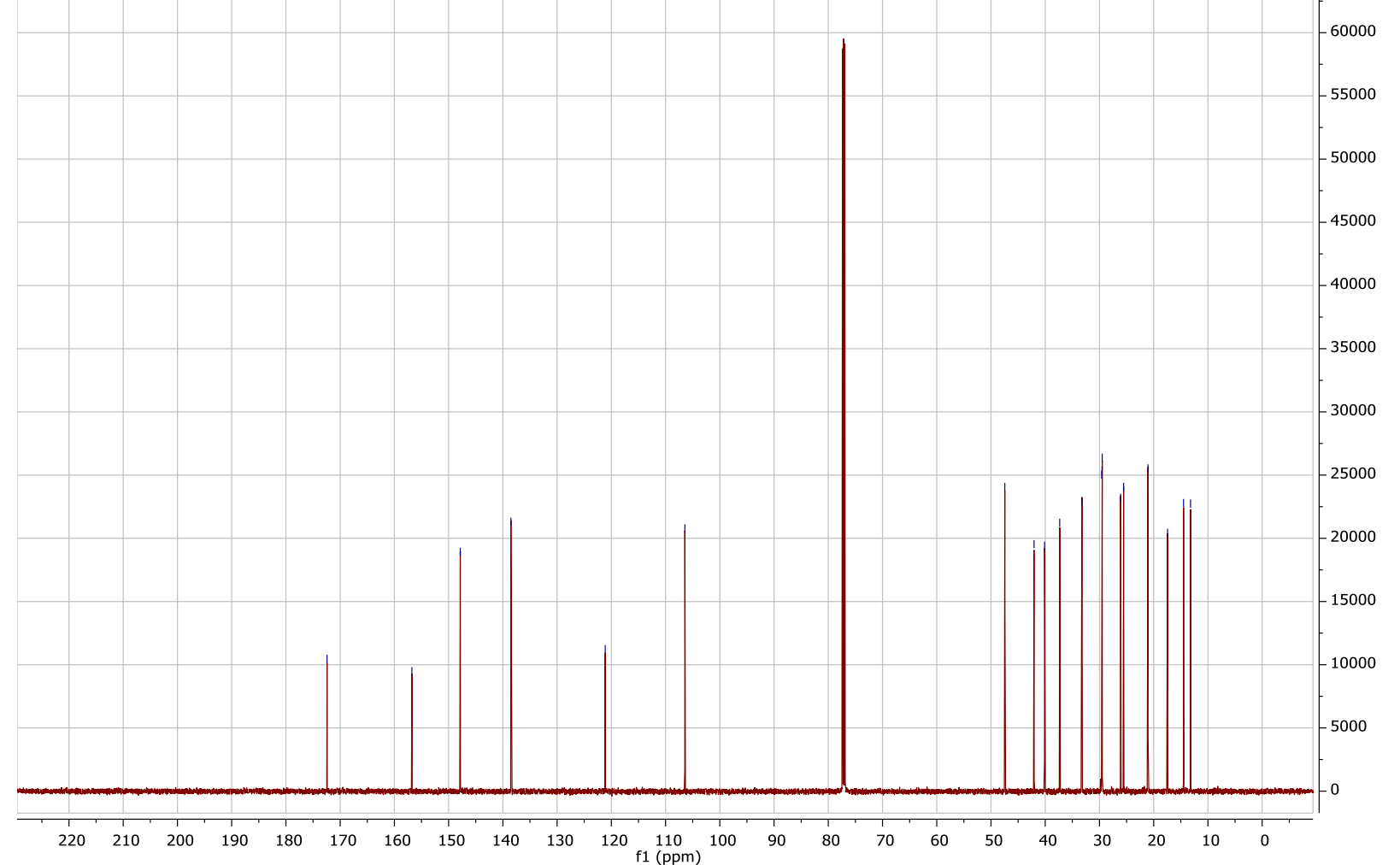


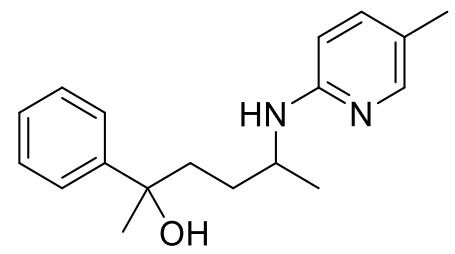

5-((5-methylpyridin-2-yl)amino)-2-phenylhexan-2-ol (8a)

${ }^{1} \mathbf{H}$

co-150-prodfin.1.fid

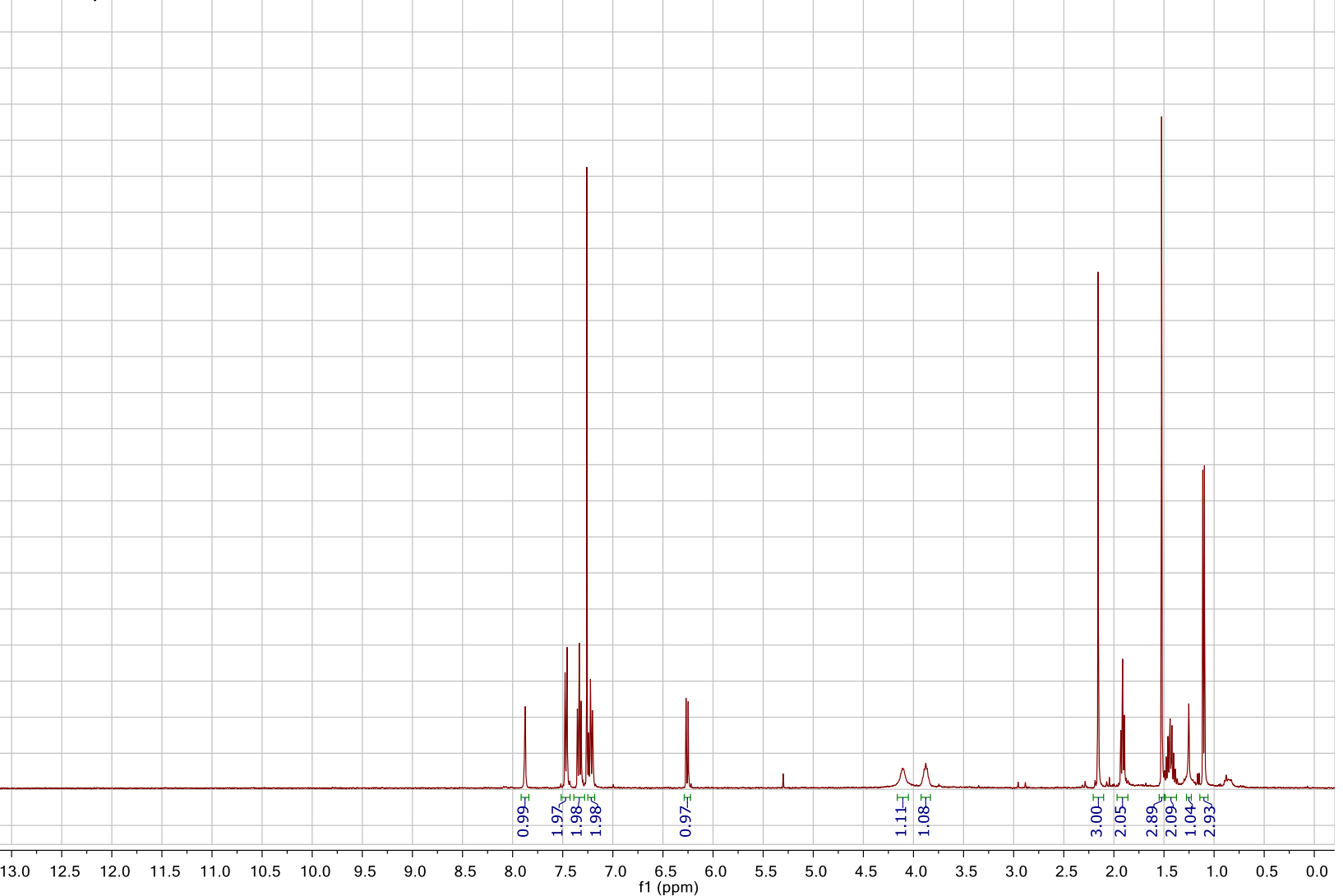


${ }^{13} \mathrm{C}$

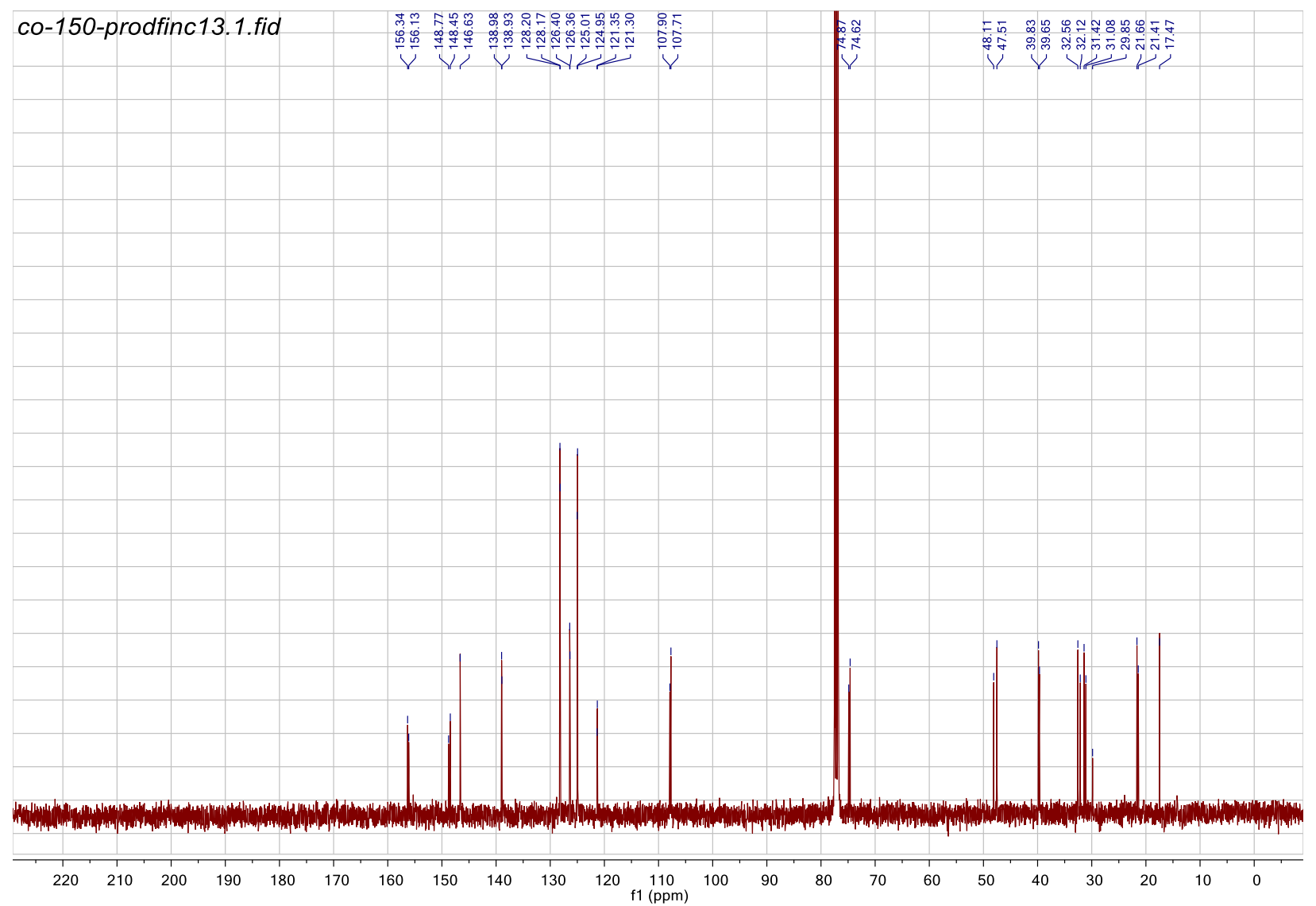


<smiles>Cc1ccc(NC(C)C2CC=CCC2)nc1</smiles>

N-(1-(cyclohex-3-en-1-yl)ethyl)-5-methylpyridin-2-amine (9a) ${ }^{1} \mathbf{H}$

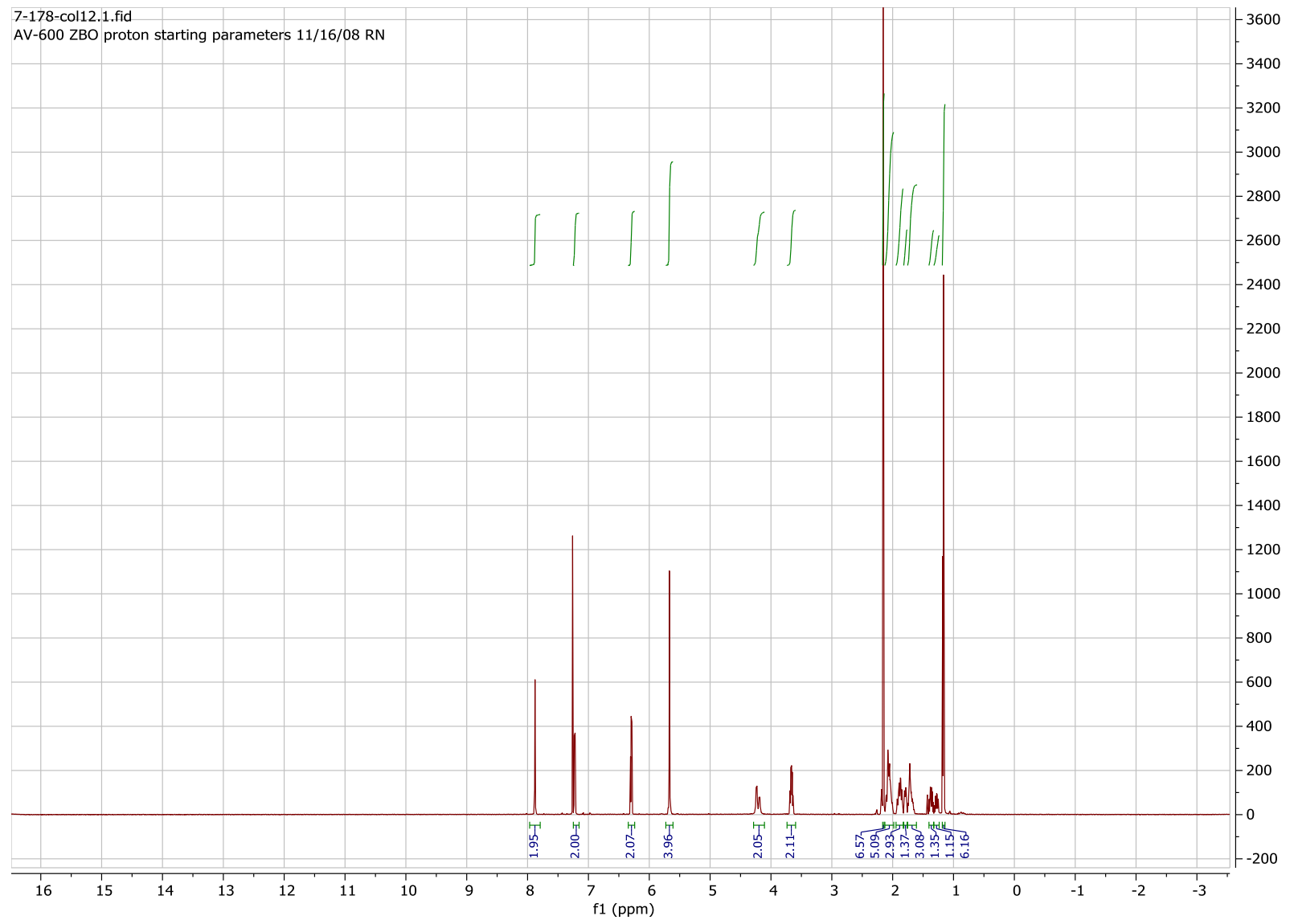


${ }^{13} \mathrm{C}$

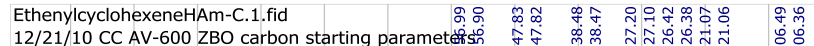

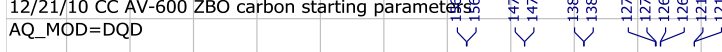

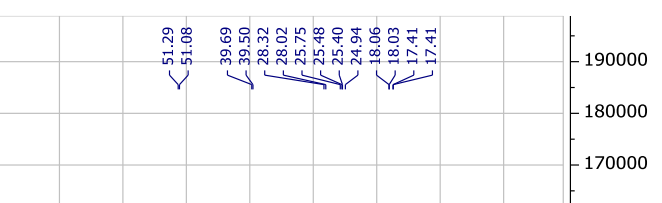

$-160000$

150000

140000

130000

120000

100000

$-90000$

$-80000$

$-70000$

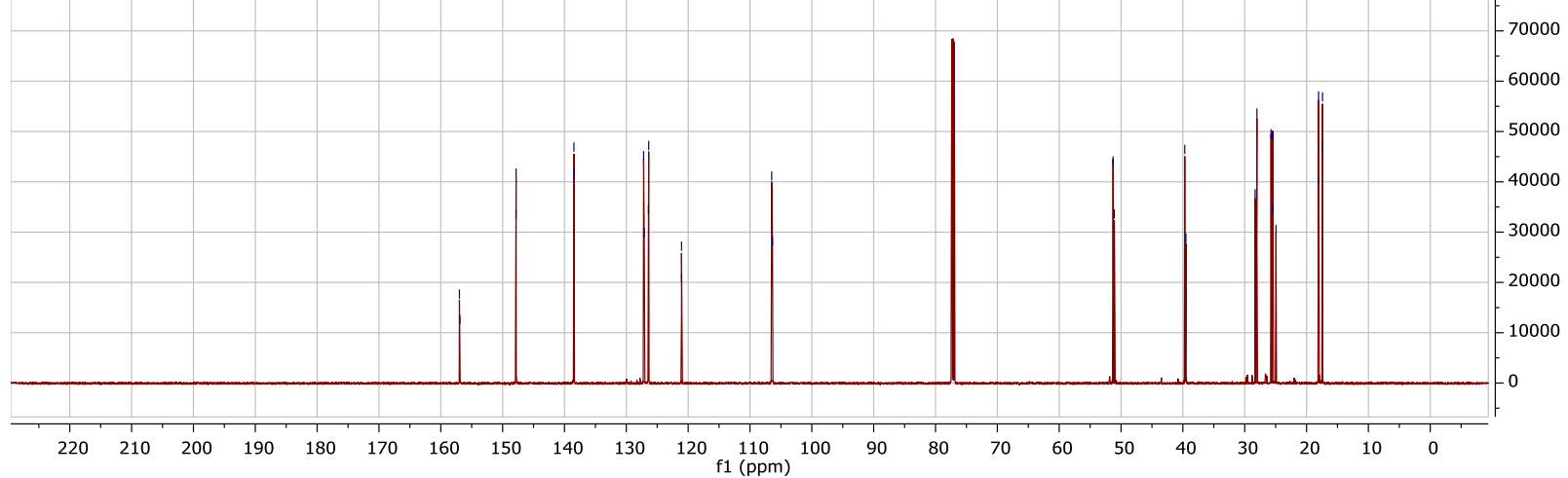




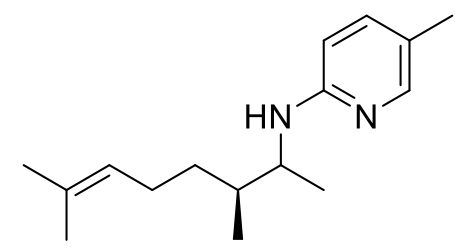

N-((3S)-3,7-dimethyloct-6-en-2-yl)-5-methylpyridin-2-amine (10a)

${ }^{1} \mathbf{H}$

betacitronelleneHAm-conc-H.1.fid

AV-600 ZBO proton starting parameters 11/16/08 RN

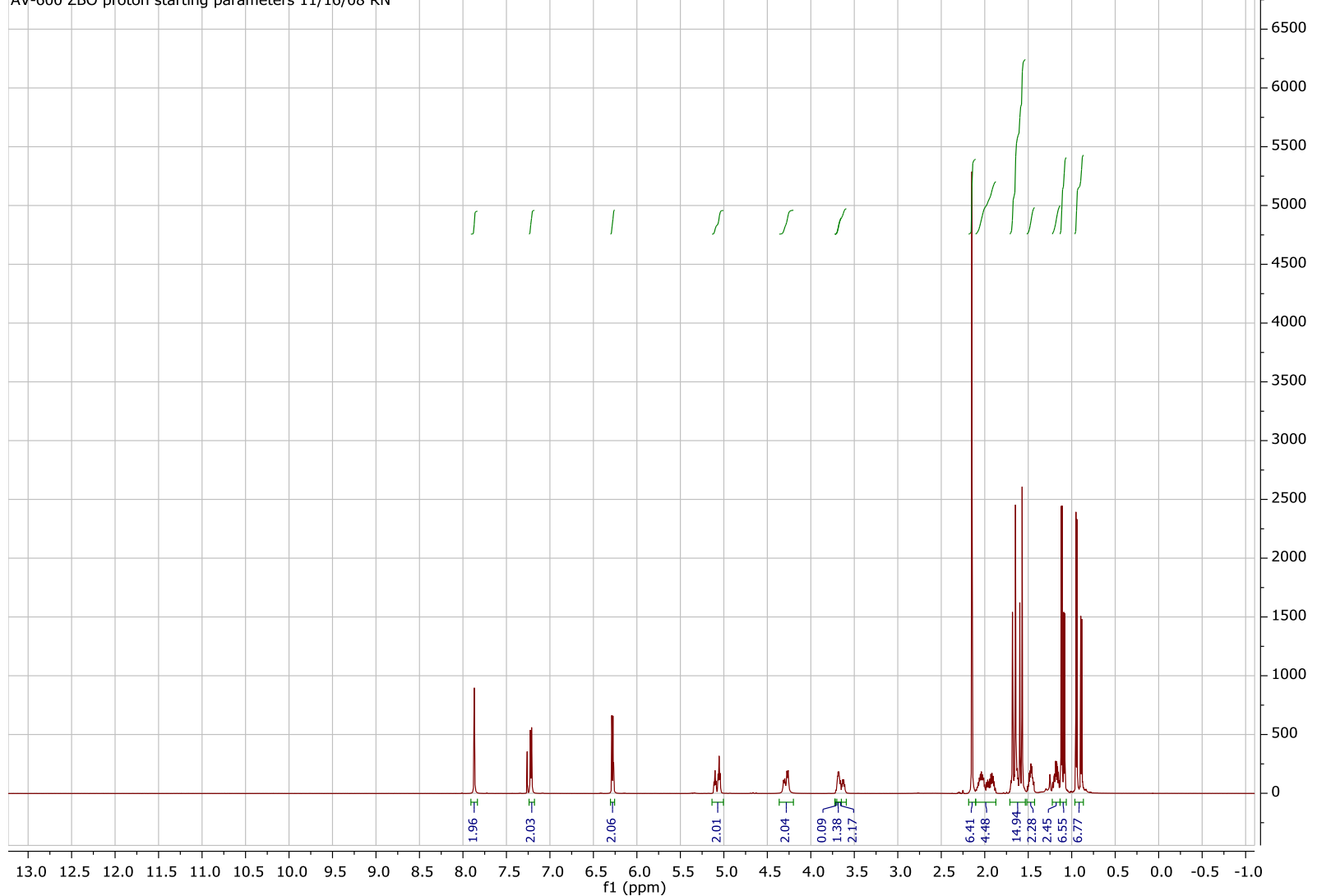


${ }^{13} \mathrm{C}$

betacitronelleneHAm-conc-C.1.fid

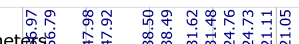

12/21/10 CC AV-600 ZBO carbon starting parametedish

$A Q \_M O D=D Q D$

$\checkmark \bar{Y} Y Y V$

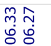

V

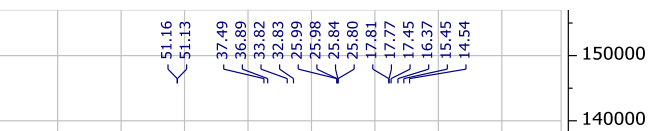

\begin{tabular}{|l|l|l|l|l|l|l|l|l|l|l|l|}
\hline & -140000 \\
\hline
\end{tabular}
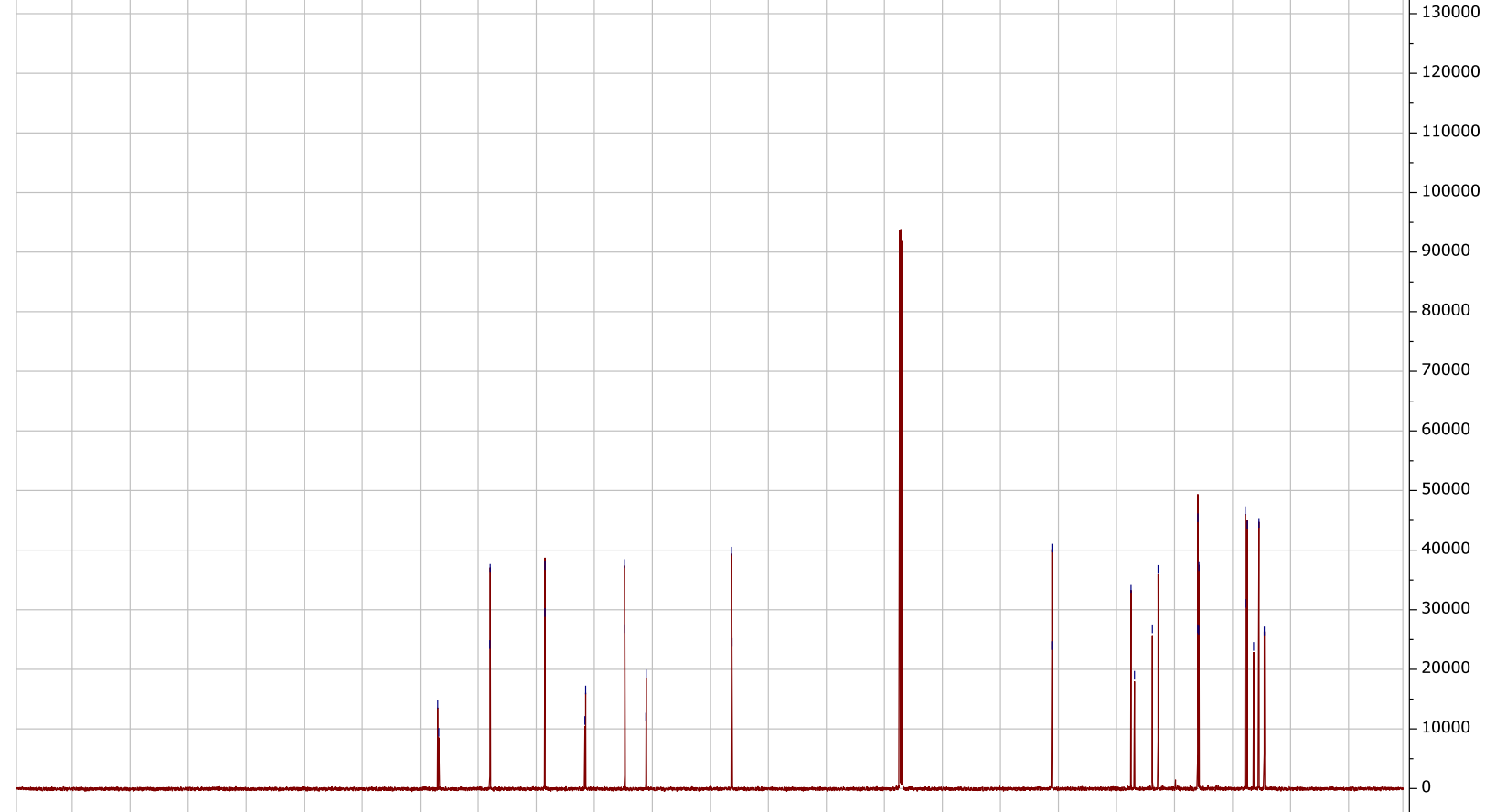

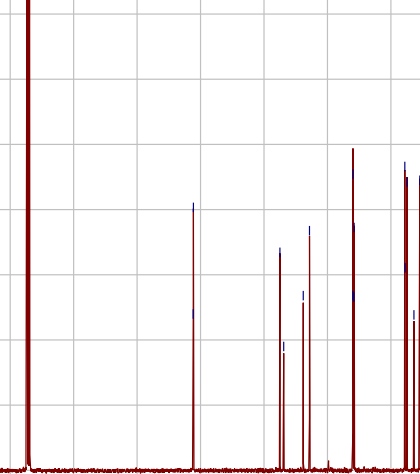

$-70000$

$-60000$

50000

$-40000$

30000

$-20000$

10000

$\begin{array}{llllllllllll}220 & 210 & 200 & 190 & 180 & 170 & 160 & 150 & 140 & 130 & 120 & 110 \\ 100\end{array}$

$90 \quad 80$

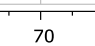

60

$\begin{array}{lllll}1 & 1 & 1 & 1 \\ 40 & 30 & 20 & 10 & 0\end{array}$ 
<smiles>C/C=C/CCC(C)Nc1ccc(C)cn1</smiles>

(E)-N-(hept-5-en-2-yl)-5-methylpyridin-2-amine (11a)

${ }^{1} \mathbf{H}$

co-14/-prod.1.tid

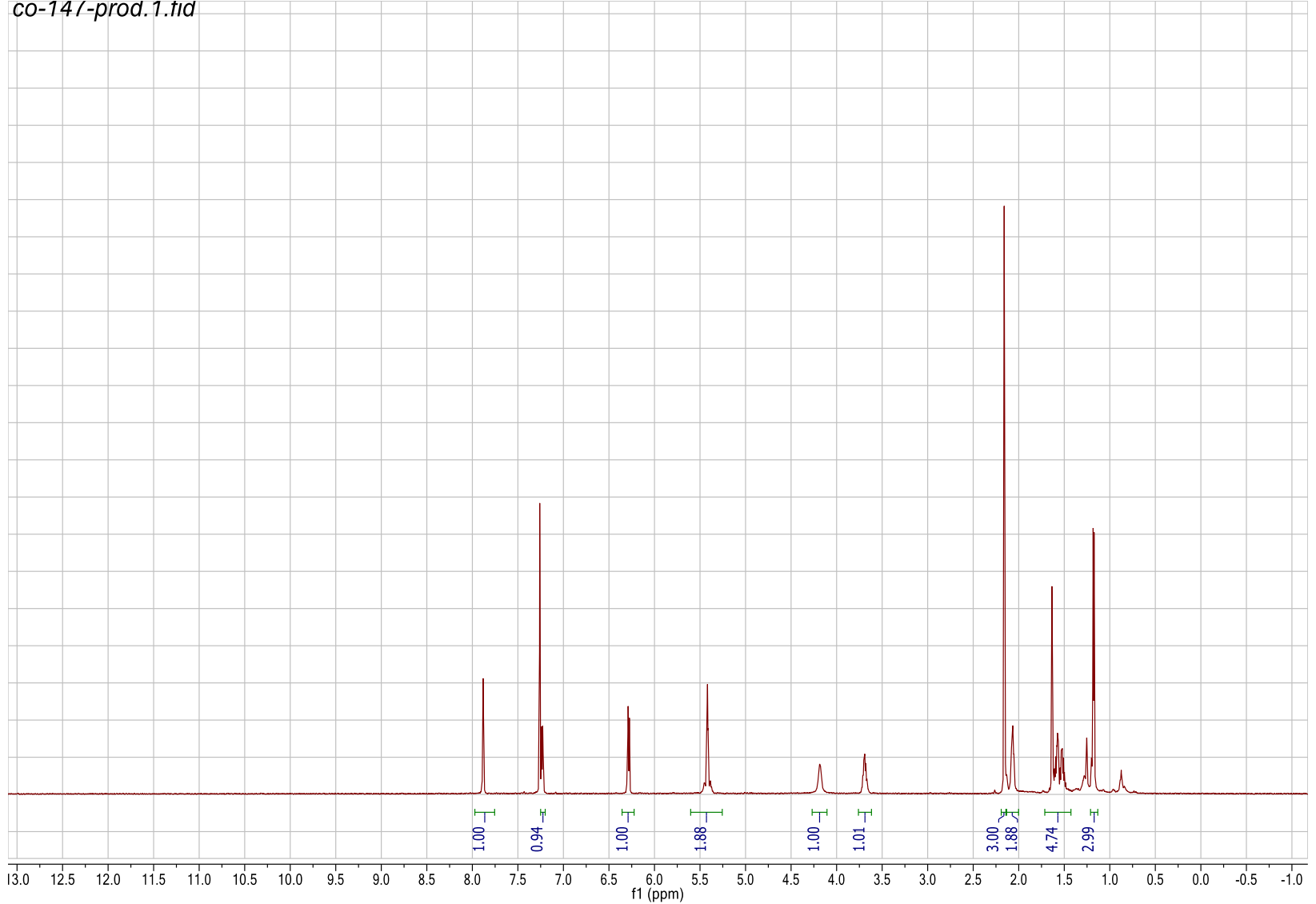


${ }^{13} \mathrm{C}$

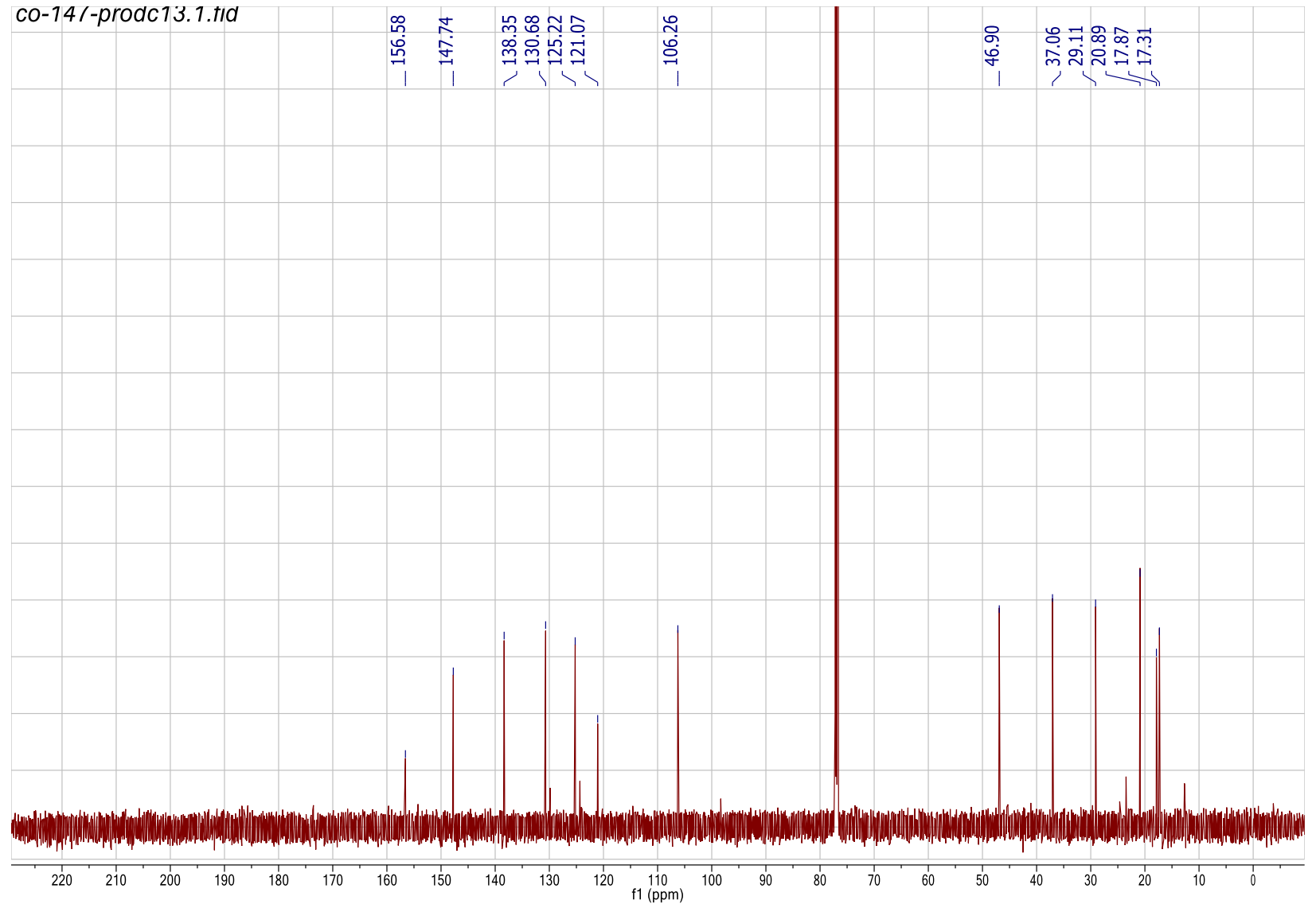


<smiles>Cc1ccc(NC(C)CCCCCCCC(=O)O[C@H]2CC[C@H]3[C@H]4CCC5=CC(=O)C=C[C@]5(C)[C@H]4CC[C@]23C)nc1</smiles>

Aminopyridyl boldenone undecylenate (12a)

${ }^{1} \mathbf{H}$

7-241-41.1.fid AV-500 BBO probe 1H 1 D NMR
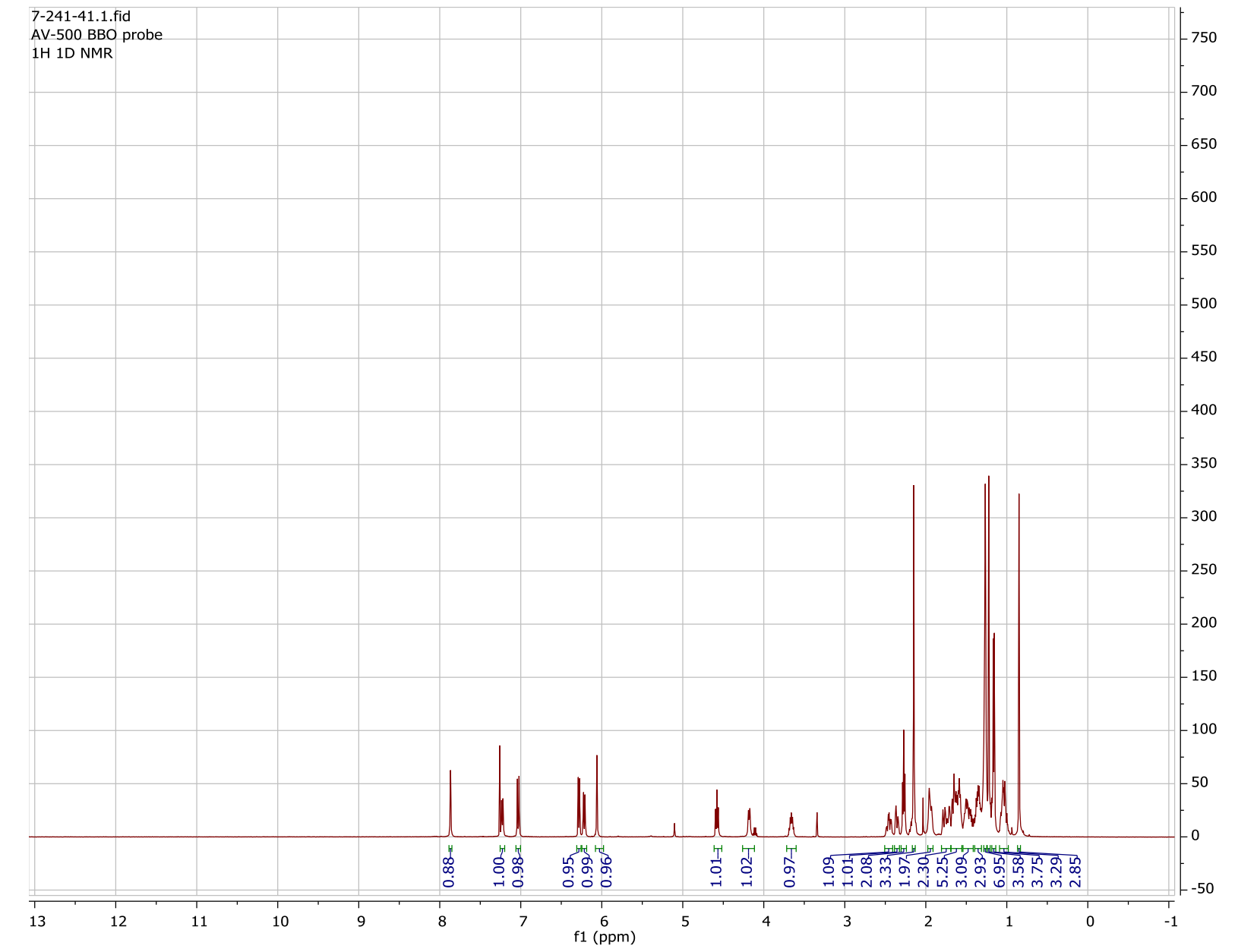
${ }^{13} \mathrm{C}$

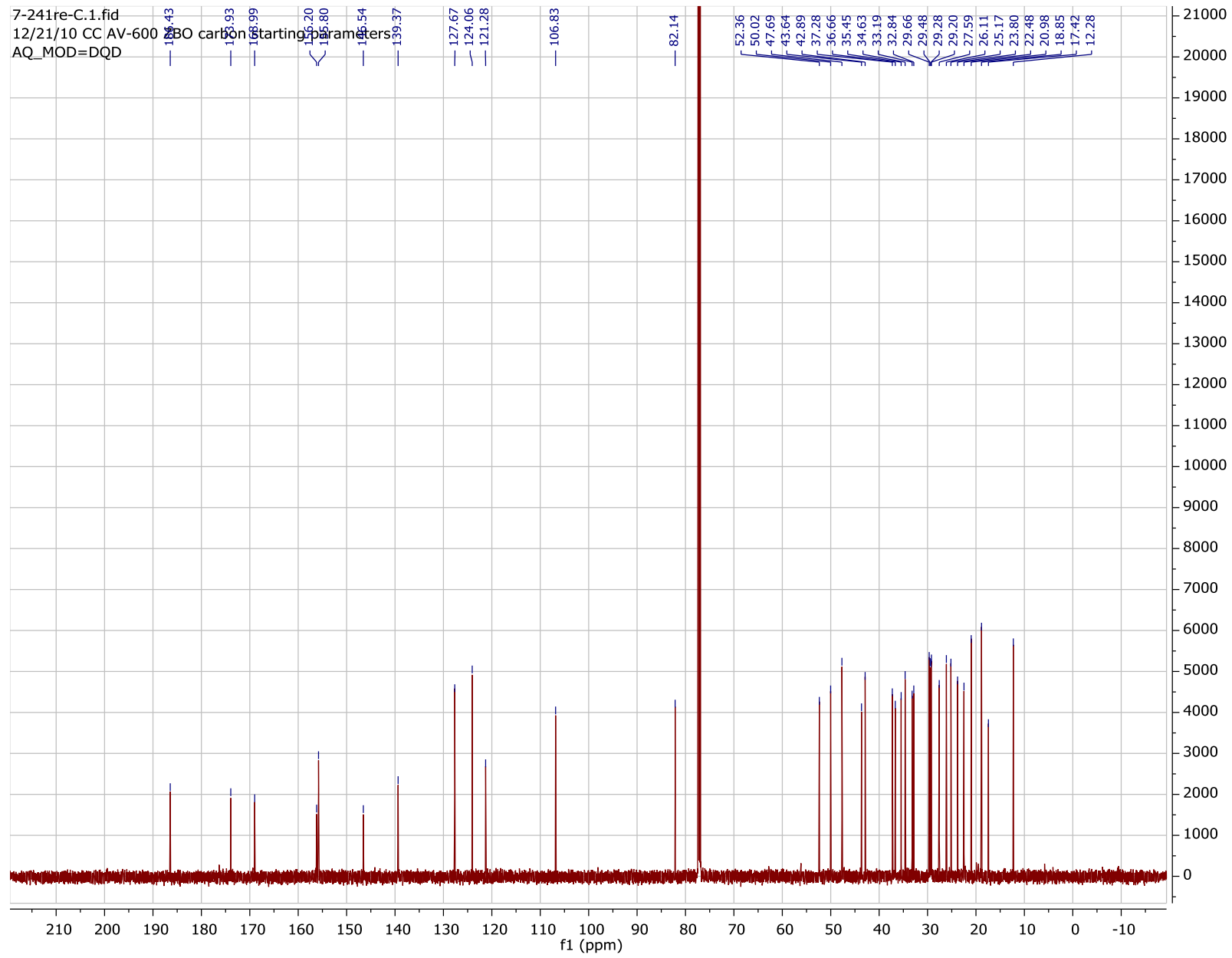


(N)

4-methyl- $N$-(dodecan-2-yl)pyridine-2-amine (13a)

${ }^{1} \mathbf{H}$

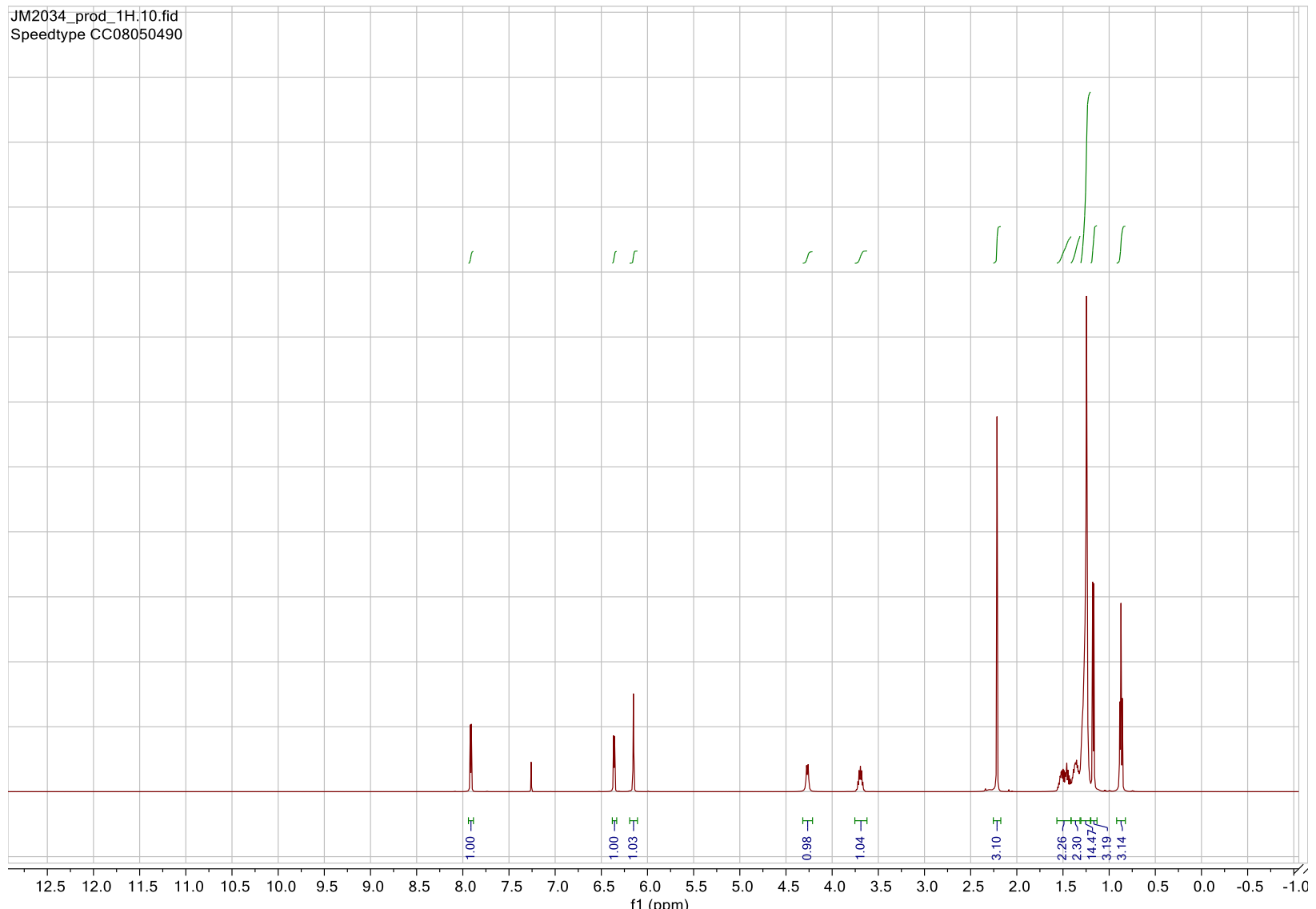




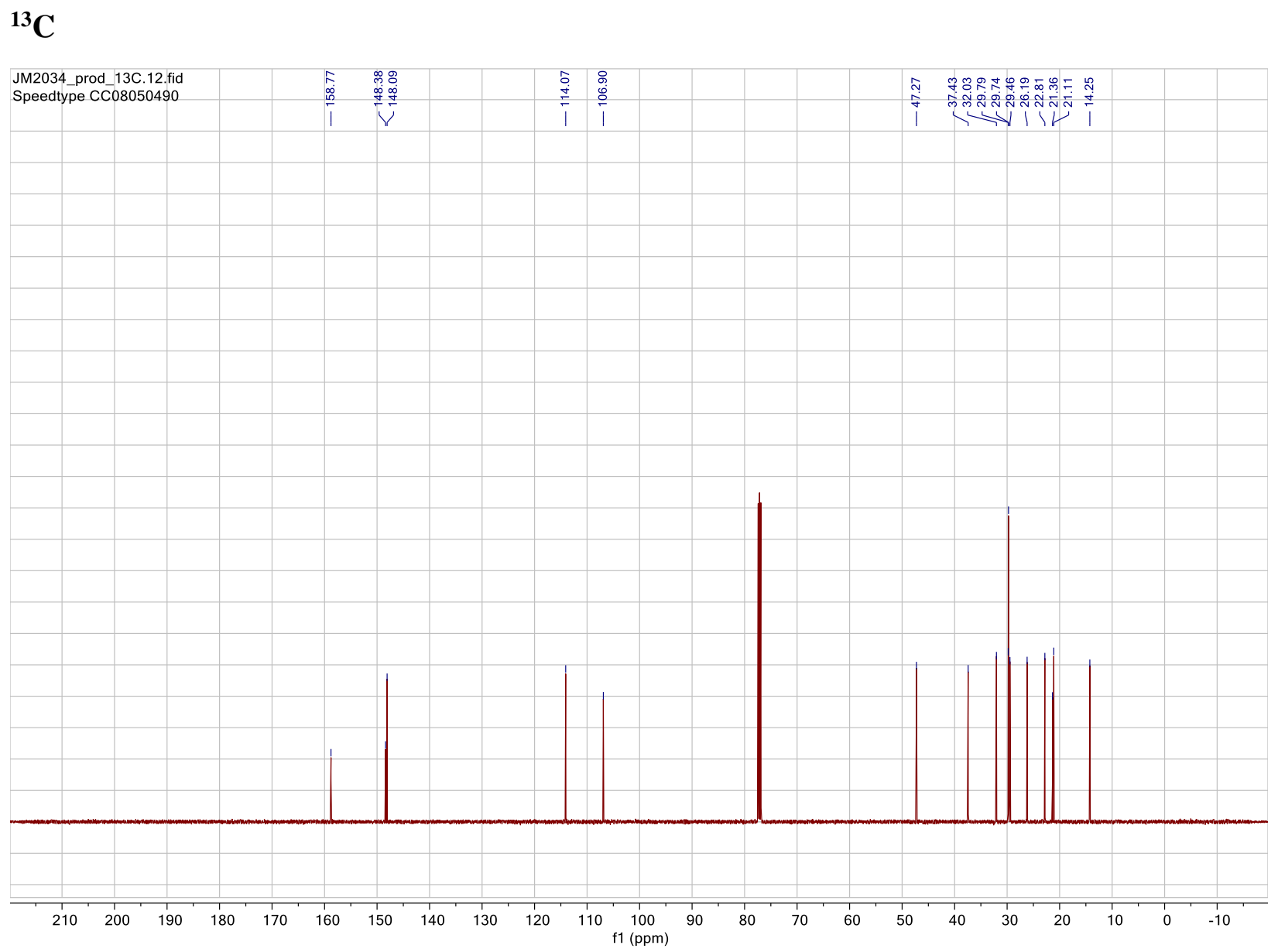


<smiles>CCCCCCC(C)Nc1ccc(OC)cn1</smiles>

\section{5-methoxy-N-(octan-2-yl)pyridin-2-amine (14a)}

${ }^{1} \mathbf{H}$

P-MeO-2-AP-octenyl-H.1.fid
AV-600 ZBO proton starting parameters $11 / 16 / 08$ RN

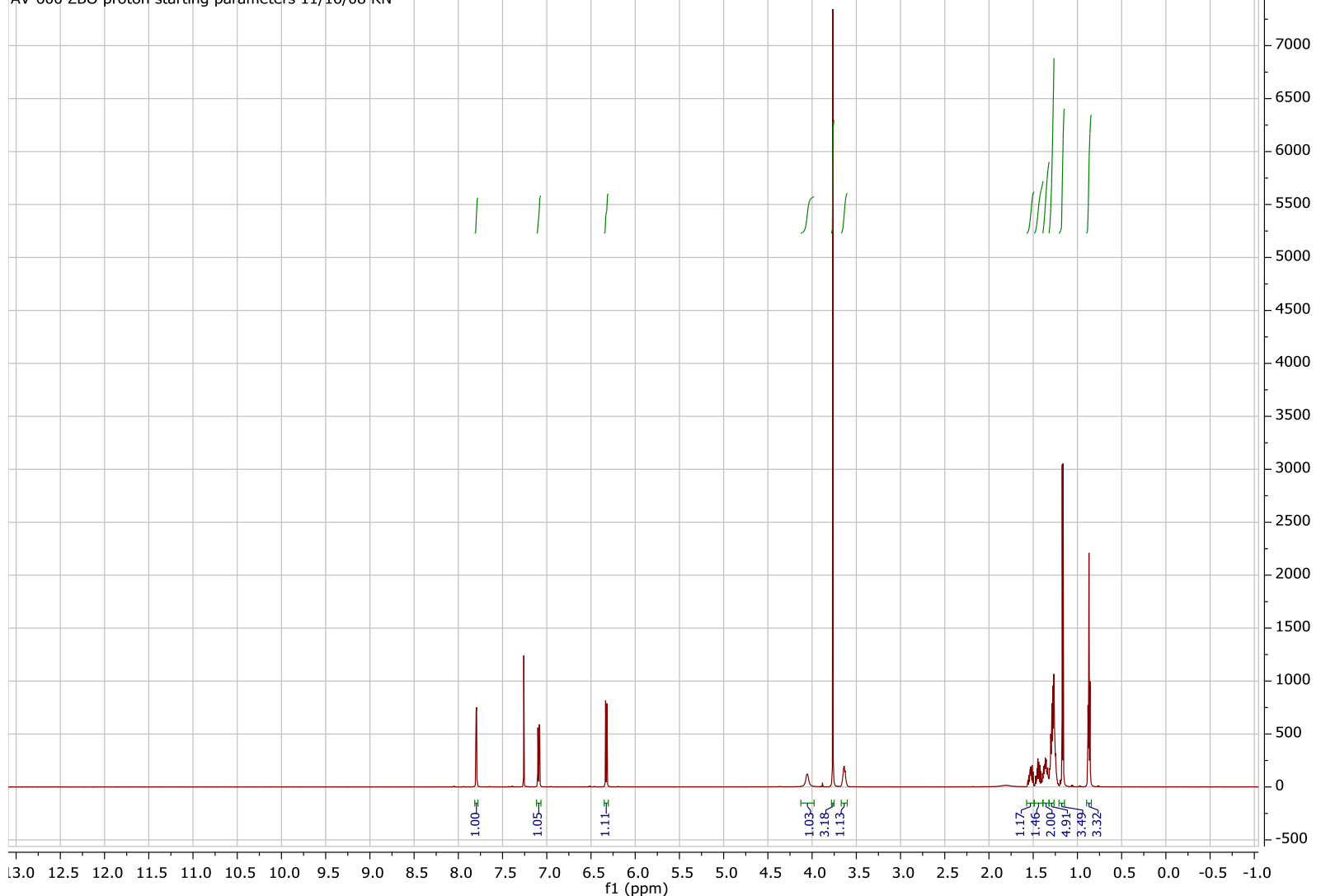


${ }^{13} \mathrm{C}$

p-MeO-2-AP-octenyl-C.1.fid
$12 / 21 / 10$ CC AV-600 ZBO carbon starting parawîn

AQ_MOD $=$ DQD
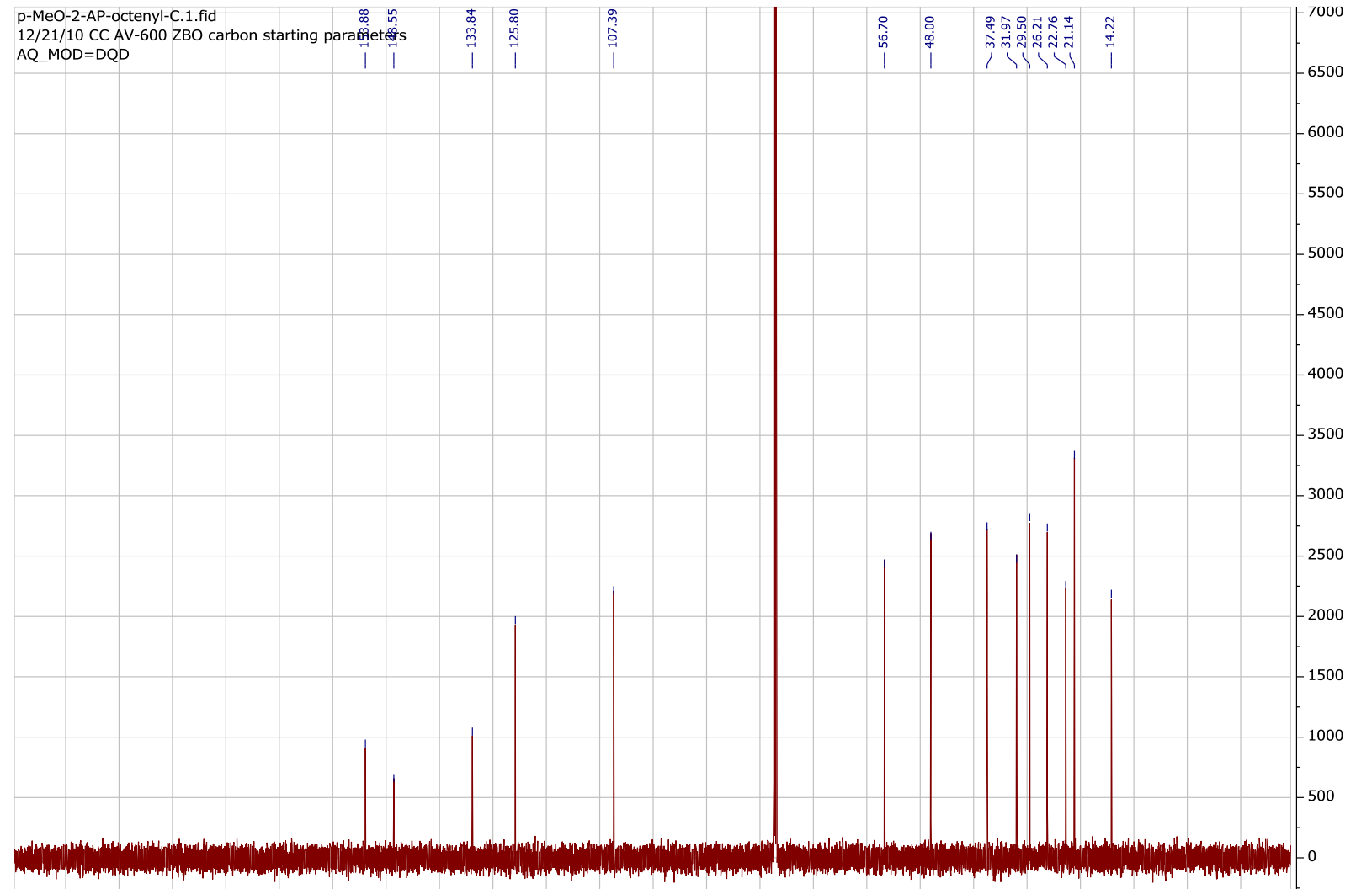

5000 4500
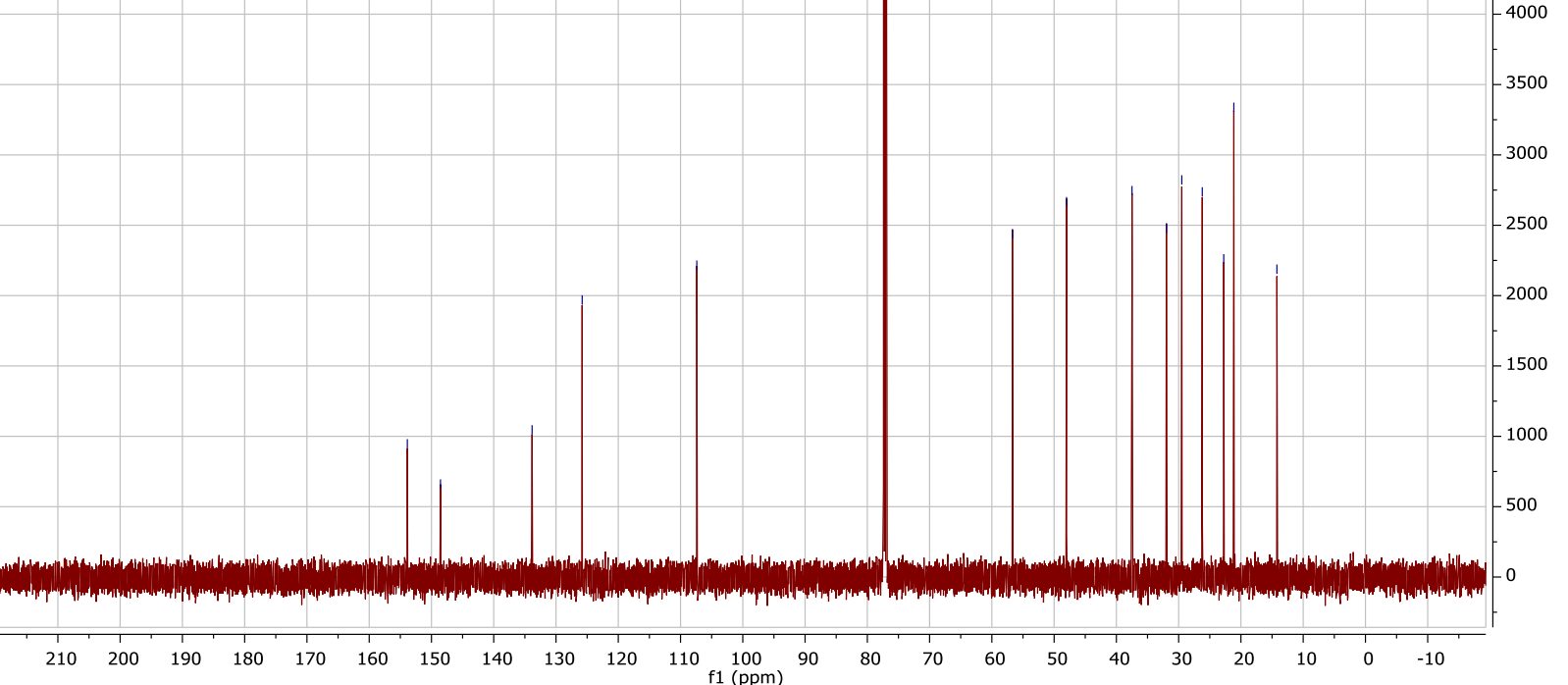


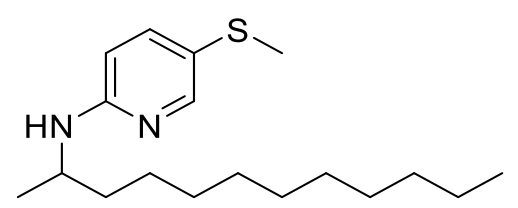

N-(dodecan-2-yl)-5-(methylthio)pyridin-2-amine (15a)

${ }^{1} \mathbf{H}$

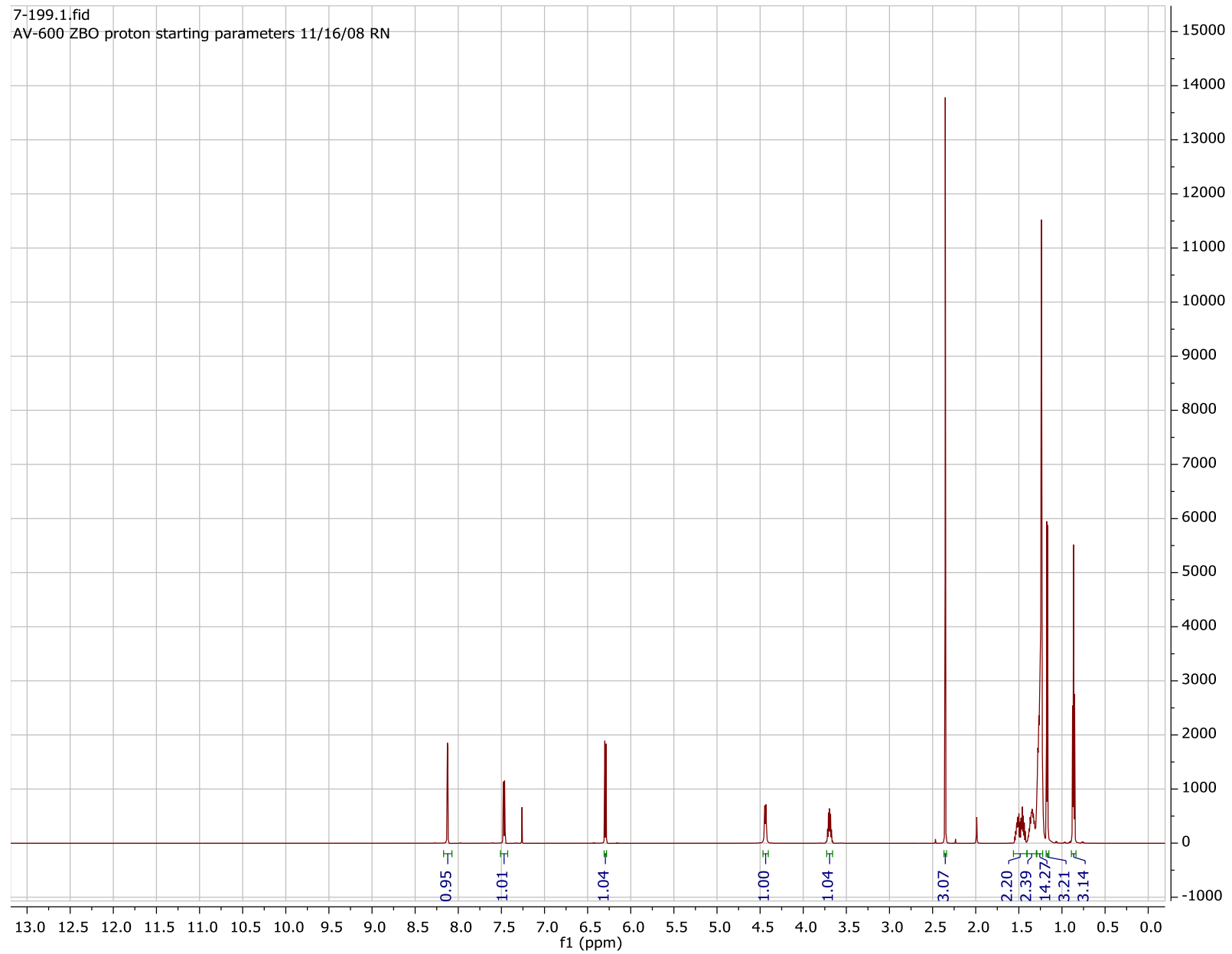


${ }^{13} \mathrm{C}$

7-199-C.1.fid
12/21/10 CC AV-600 ZBO carbon starting parameter

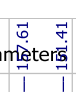

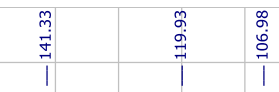

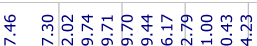

$A Q \quad M O D=D Q D$

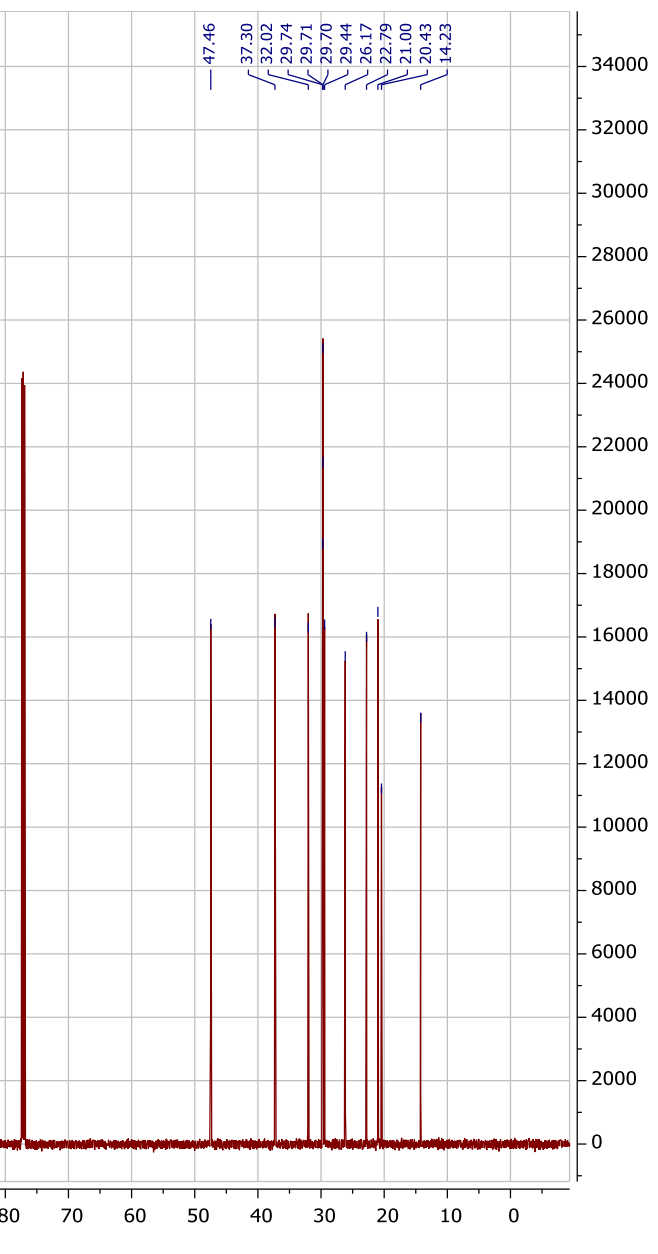


<smiles>CCCCCCCCCCC(C)Nc1ccc(N2CCN(C)CC2)cn1</smiles>

$\mathrm{N}$-(dodecan-2-yl)-5-(4-methylpiperazin-1-yl)pyridin-2-amine (16a) ${ }^{1} \mathbf{H}$

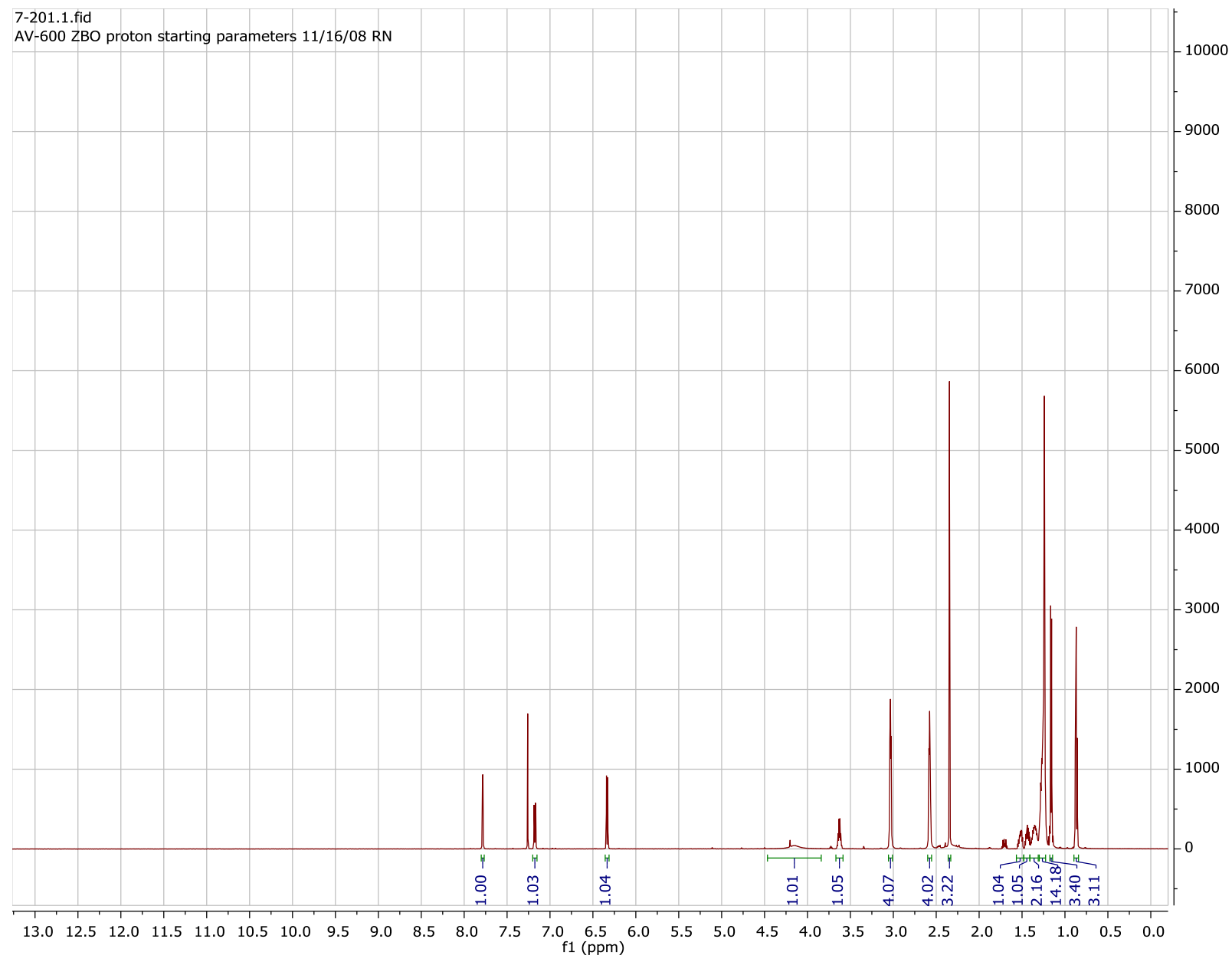


${ }^{13} \mathrm{C}$

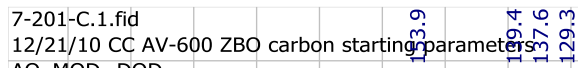

AQ_MOD $=D Q D$

$\underset{-1}{\stackrel{1}{0}}$

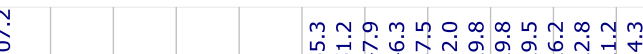

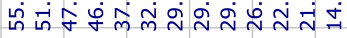

$11 /<>$

45000

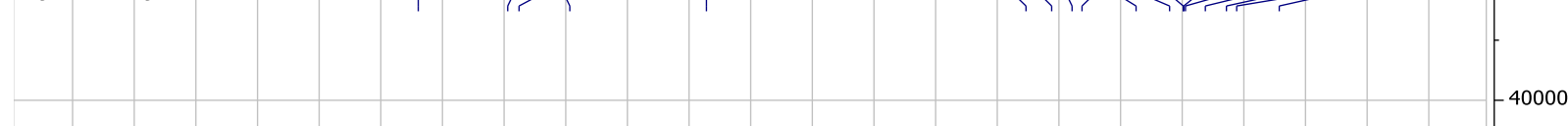

\begin{tabular}{|l|l|l|l|l|l|l|l|l|l|l|l|l|l|l|l|l|l|}
\hline & & & & & \\
\hline
\end{tabular}
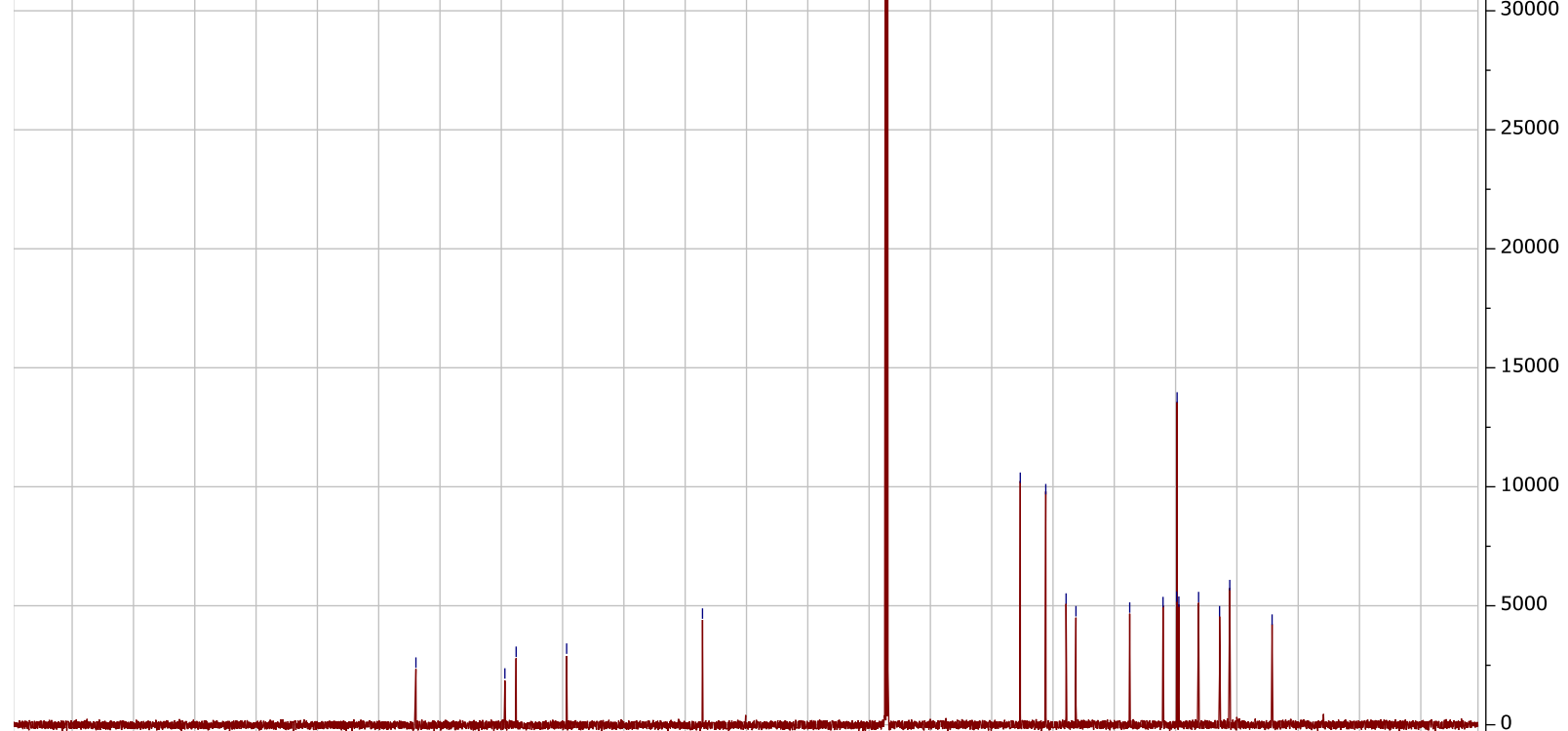

- 
N-(1-cyclohexylethyl)-5-methylpyridin-2-amine (17a)<smiles>Cc1ccnc(NC(C)C2CCCCC2)c1</smiles>

${ }^{1} \mathbf{H}$

JM_vinylcyclohexane_prod_1H.1.fid

1H 1D NMR

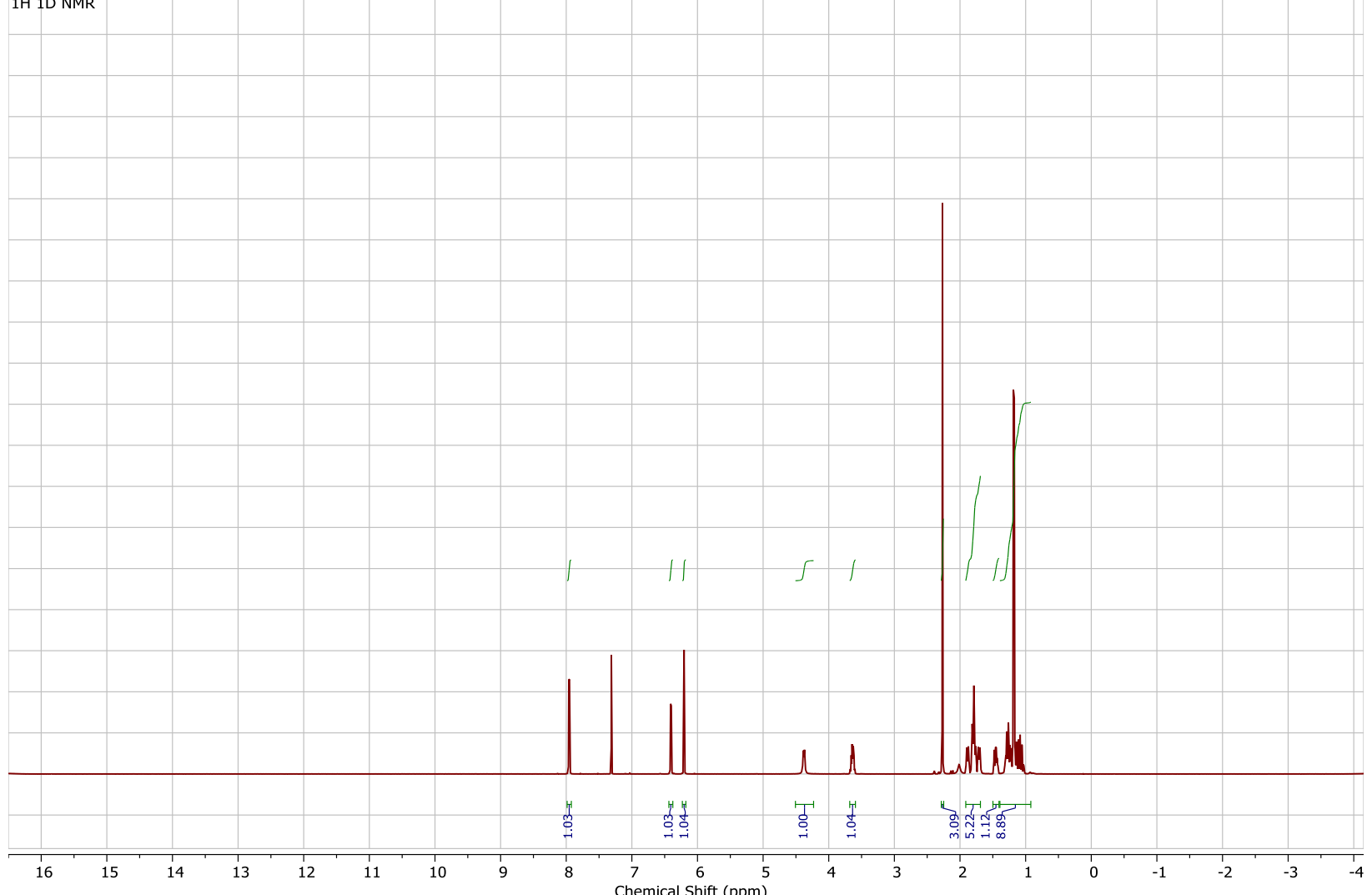




\section{${ }^{13} \mathrm{C}$}

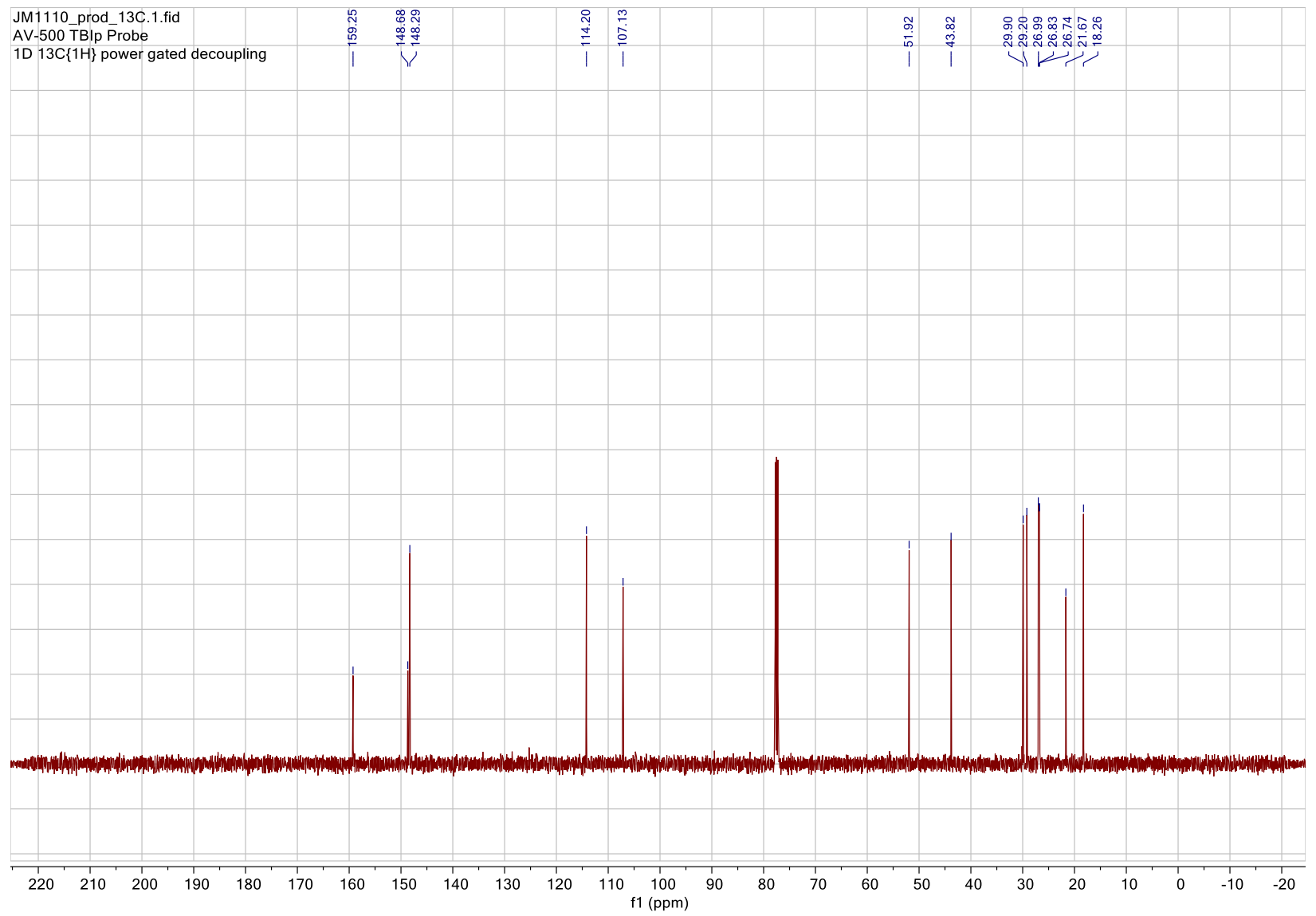


<smiles>Cc1ccnc(NC(C)c2ccccc2)c1</smiles>

\section{4-methyl- $N$-(1-phenylethyl)pyridin-2-amine (18a)}

\section{${ }^{1} \mathbf{H}$}

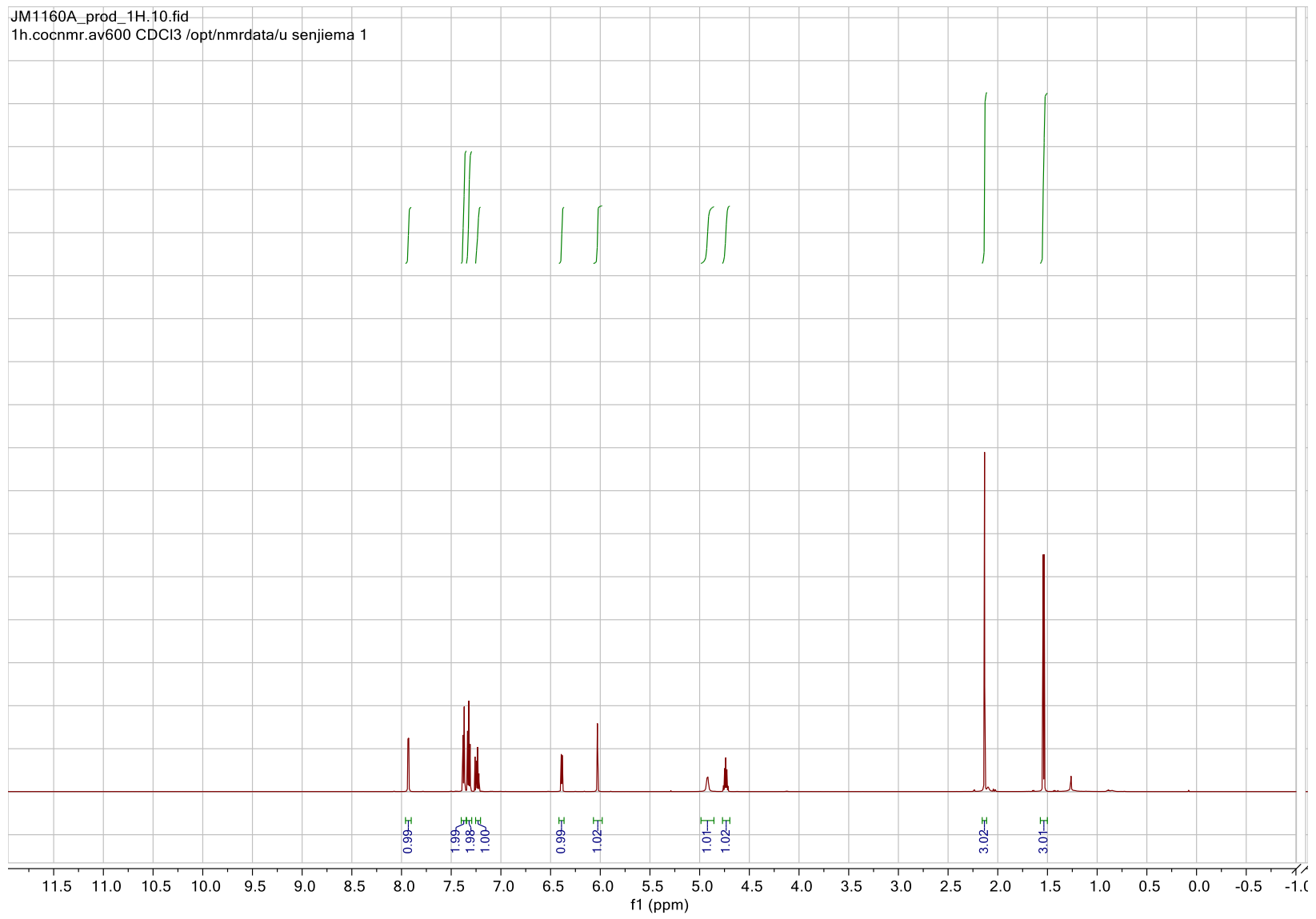


${ }^{13} \mathrm{C}$

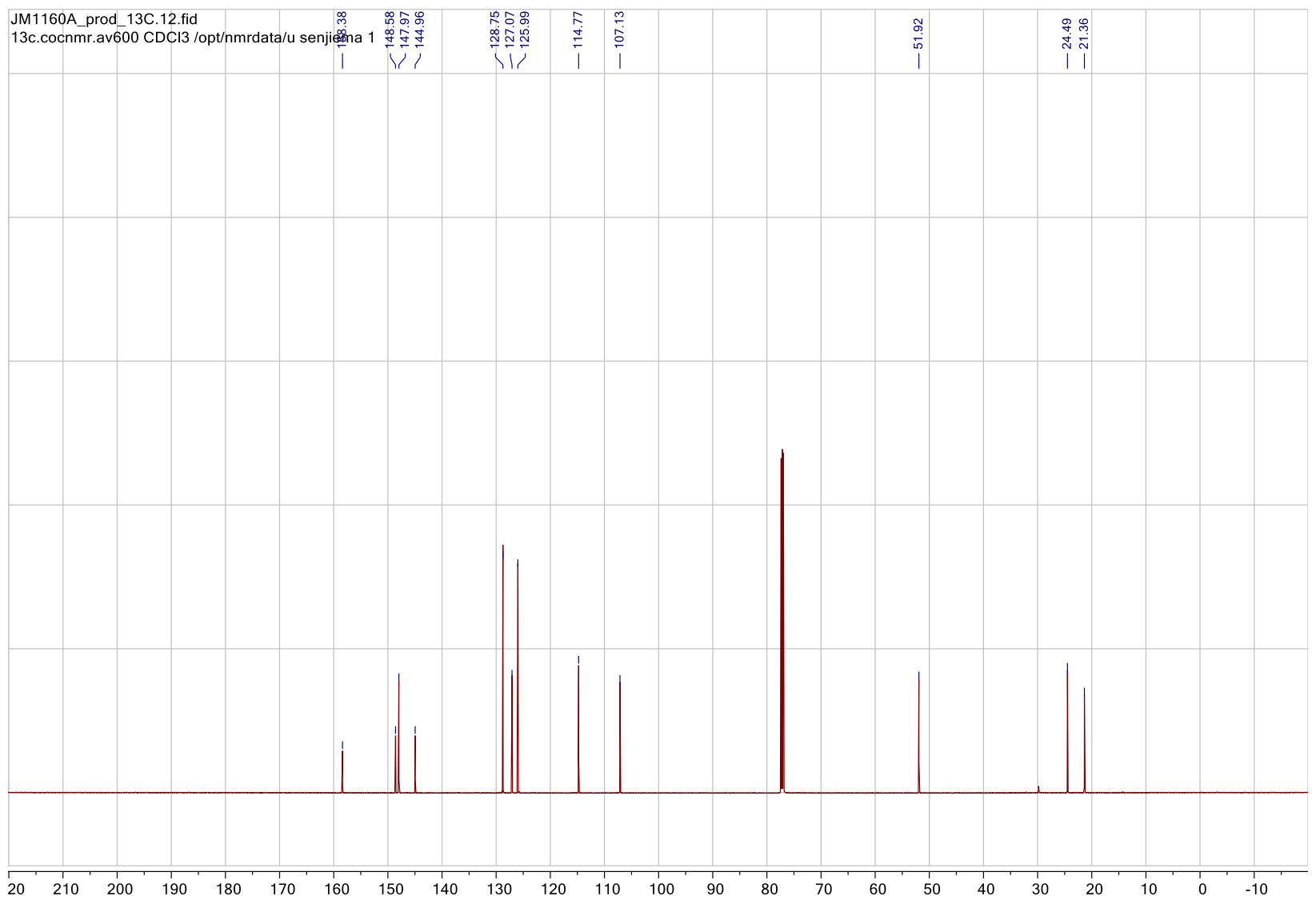

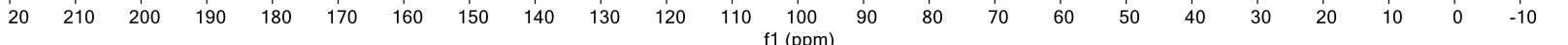


<smiles>Cc1ccc(C(C)Nc2cc(C)ccn2)cc1</smiles>

\section{4-methyl-N-(1-(p-tolyl)ethyl)pyridin-2-amine (19a)}

\section{${ }^{1} \mathbf{H}$}

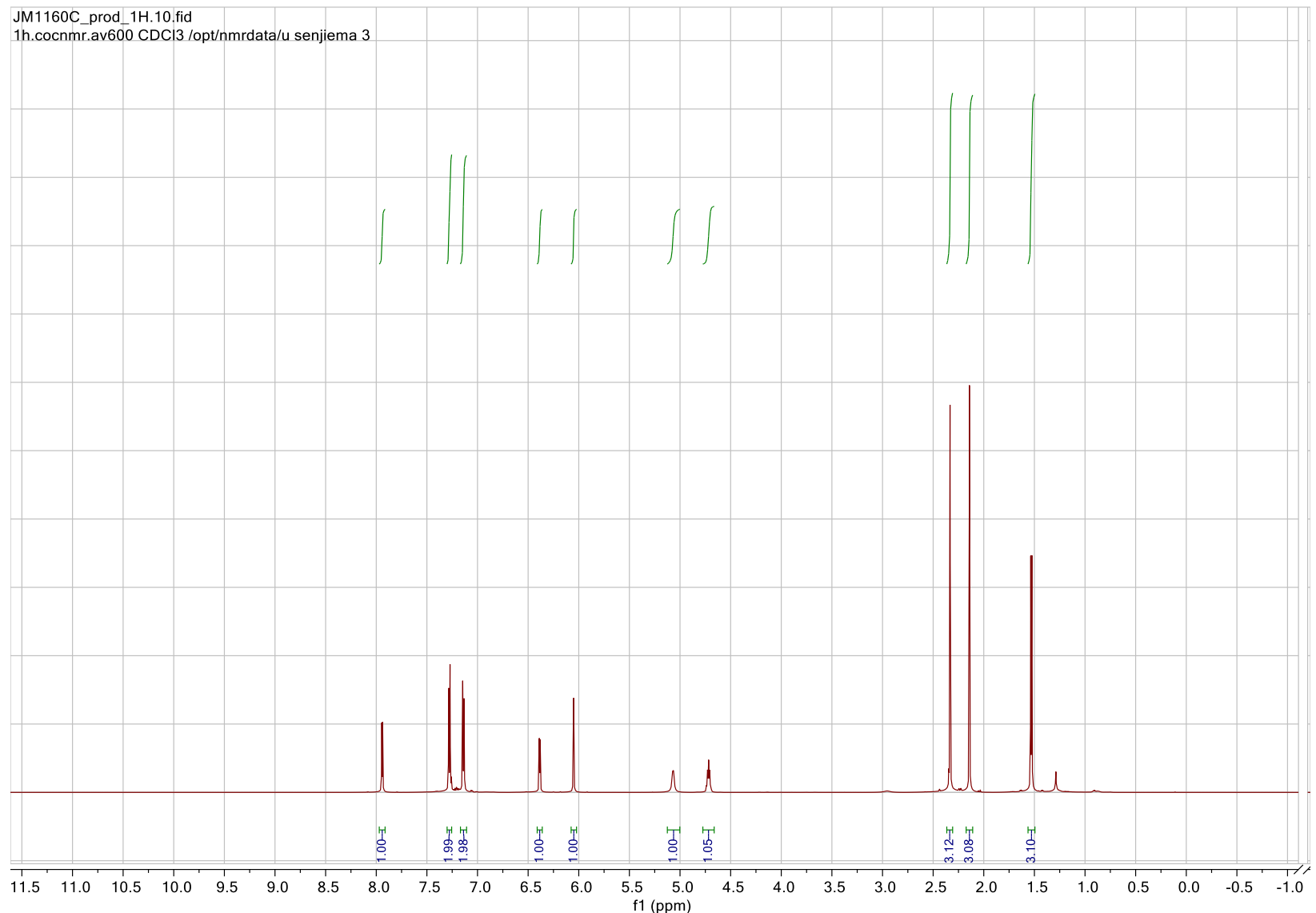


${ }^{13} \mathrm{C}$

JM2012_PMe_13C.13.fid Speedtype CC08050490

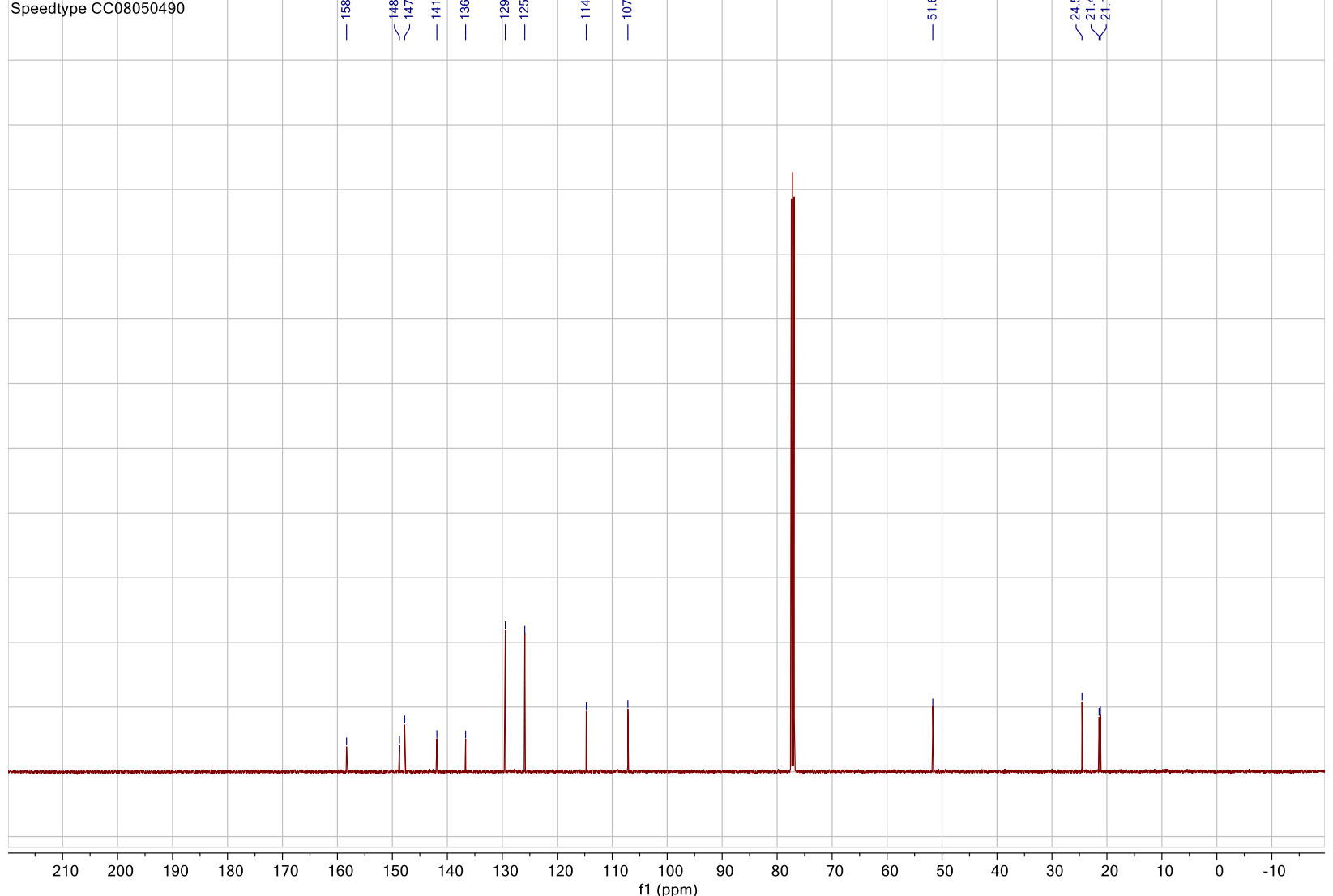


<smiles>Cc1ccnc(NC(C)c2ccc(F)cc2)c1</smiles>

N-(1-(4-fluorophenyl)ethyl)-4-methylpyridin-2-amine (20a)

\section{${ }^{1} \mathbf{H}$}

JM1160B_prod_1H.10.fid

1h.cocn

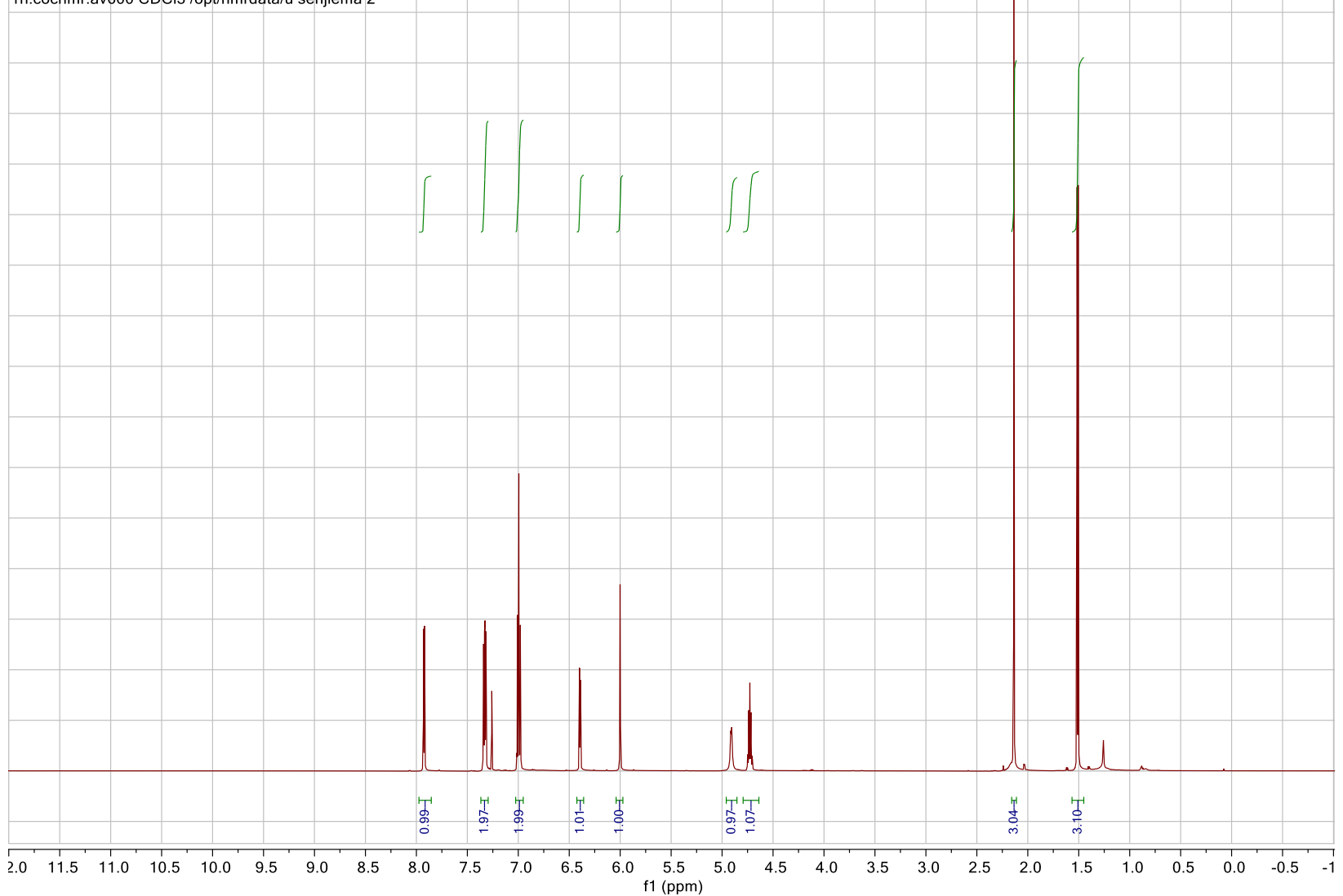


${ }^{13} \mathrm{C}$

JM1160B_prod_13C.15.fid

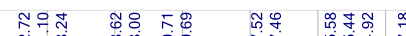

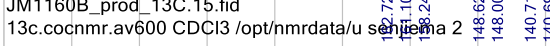

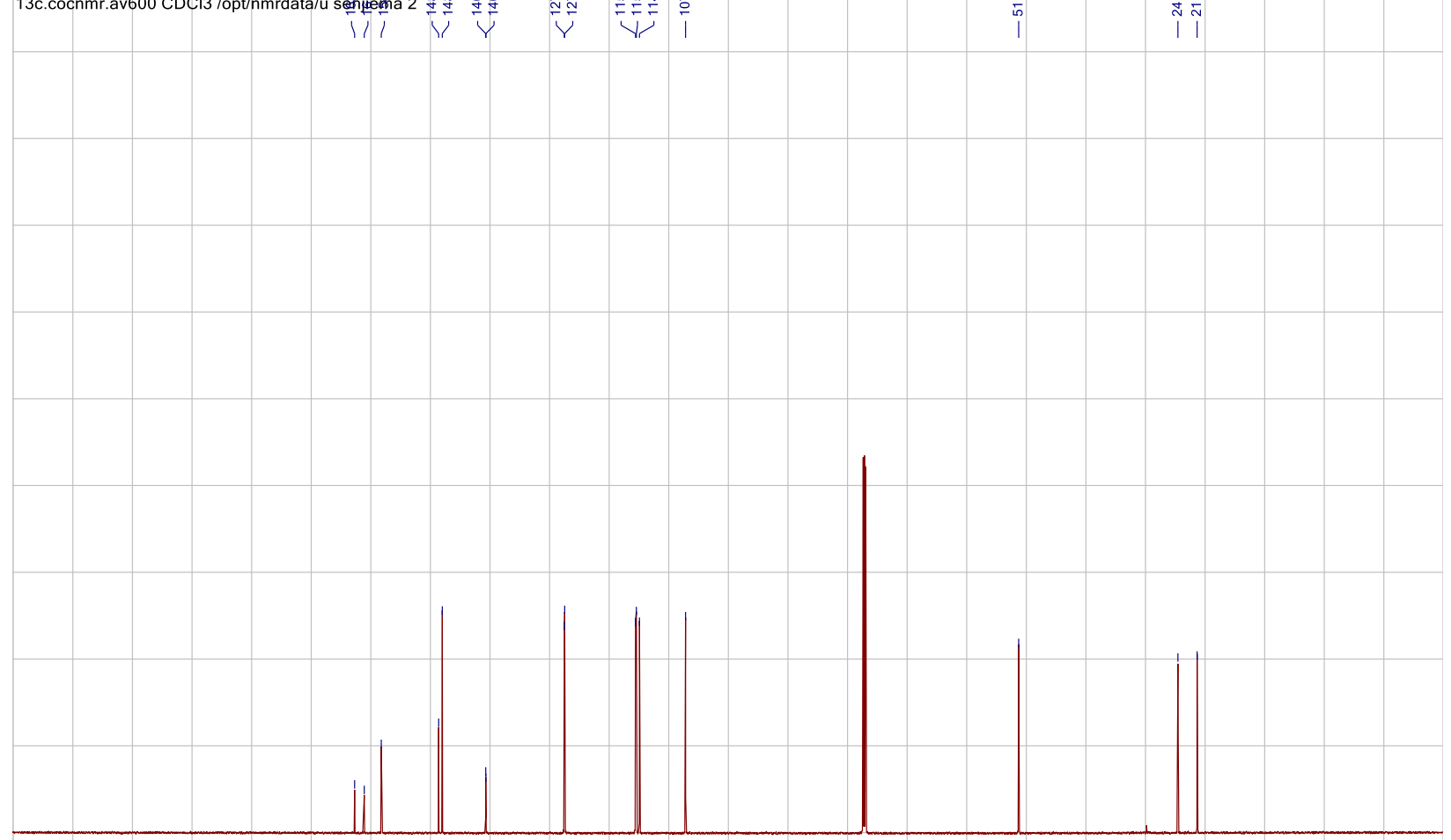

$\begin{array}{llllllllllllllllllllllll}20 & 210 & 200 & 190 & 180 & 170 & 160 & 150 & 140 & 130 & 120 & 110 & 100 & 90 & 80 & 70 & 60 & 50 & 40 & 30 & 20 & 10 & 0 & -10\end{array}$ 


\section{${ }^{19} \mathbf{F}$}

JM1160B_prod_19f.13.fid

19f.cocnmr.av600 CDCl3/opt/nmrdata/u senjiema 2

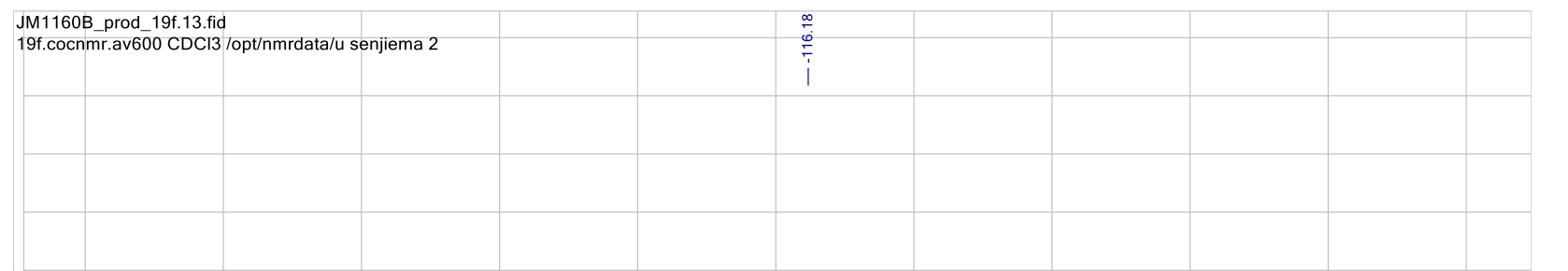

\begin{tabular}{|l|l|l|l|l|l|}
\hline & & & & \\
\hline & & & & \\
\hline
\end{tabular}

\begin{tabular}{|l|l|l|}
\hline & & \\
\hline & & \\
\hline
\end{tabular}

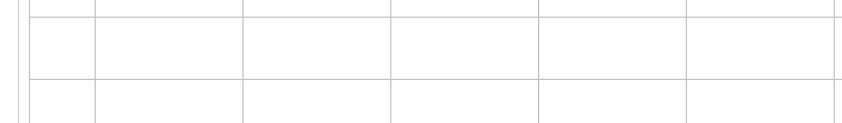

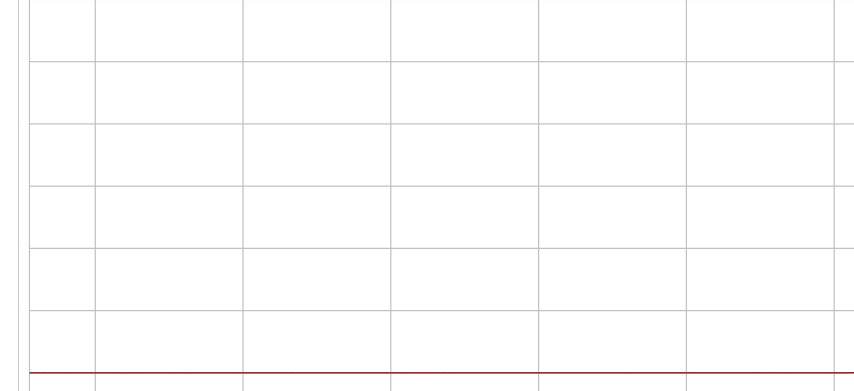

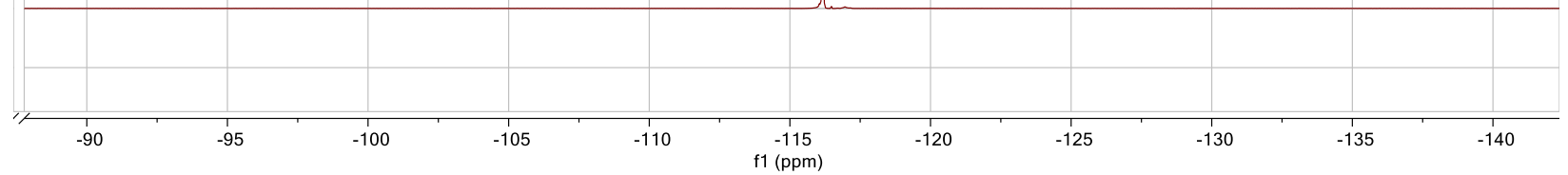


<smiles>COc1ccc(C(C)Nc2cc(C)ccn2)cc1</smiles>

N-(1-(4-methoxyphenyl)ethyl)-4-methylpyridin-2-amine (21a)

\section{${ }^{1} \mathbf{H}$}

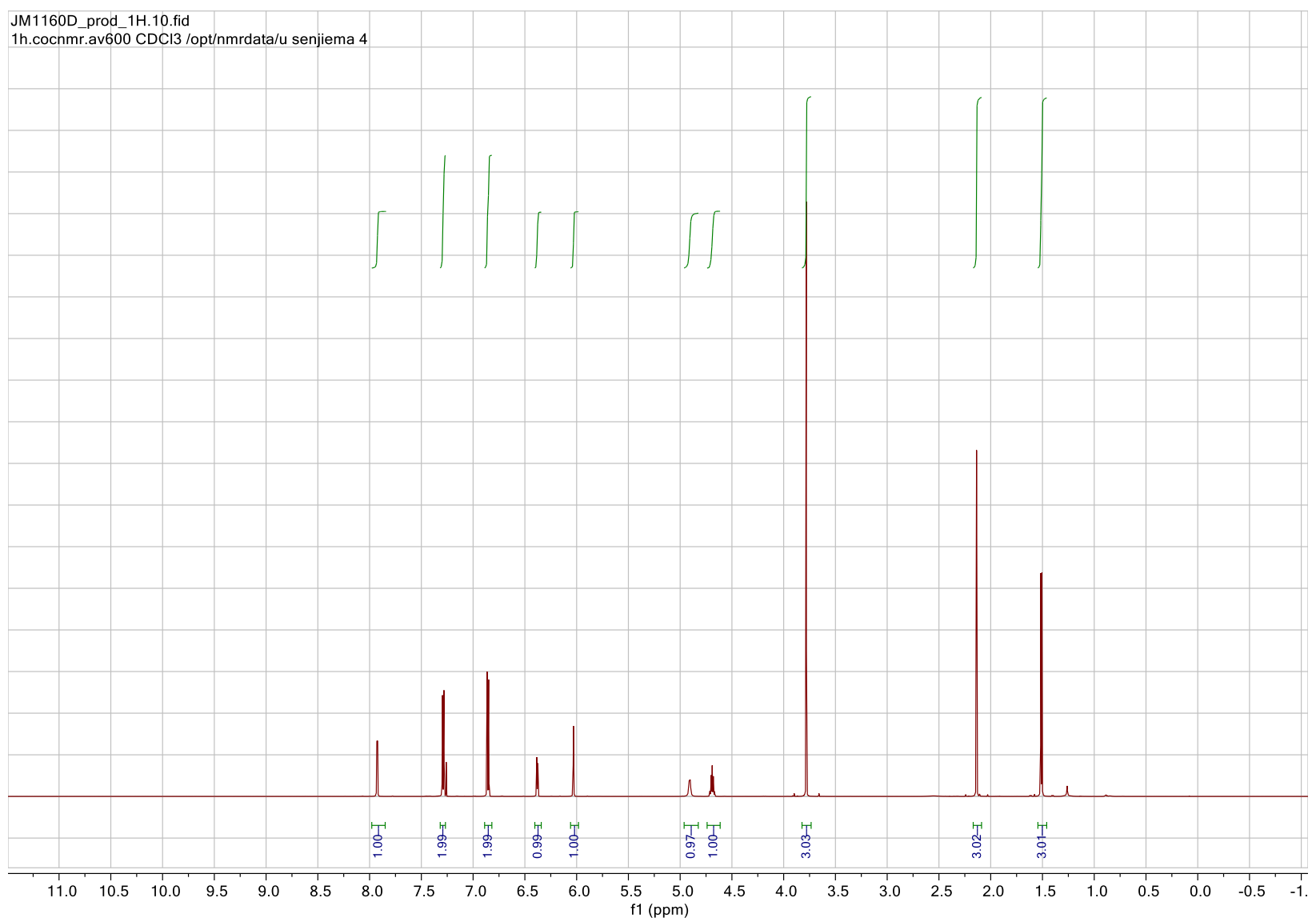


${ }^{13} \mathrm{C}$

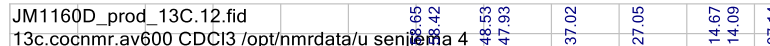

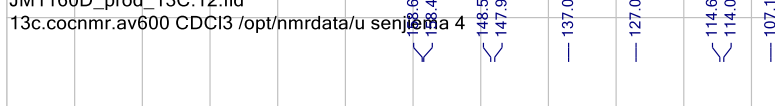
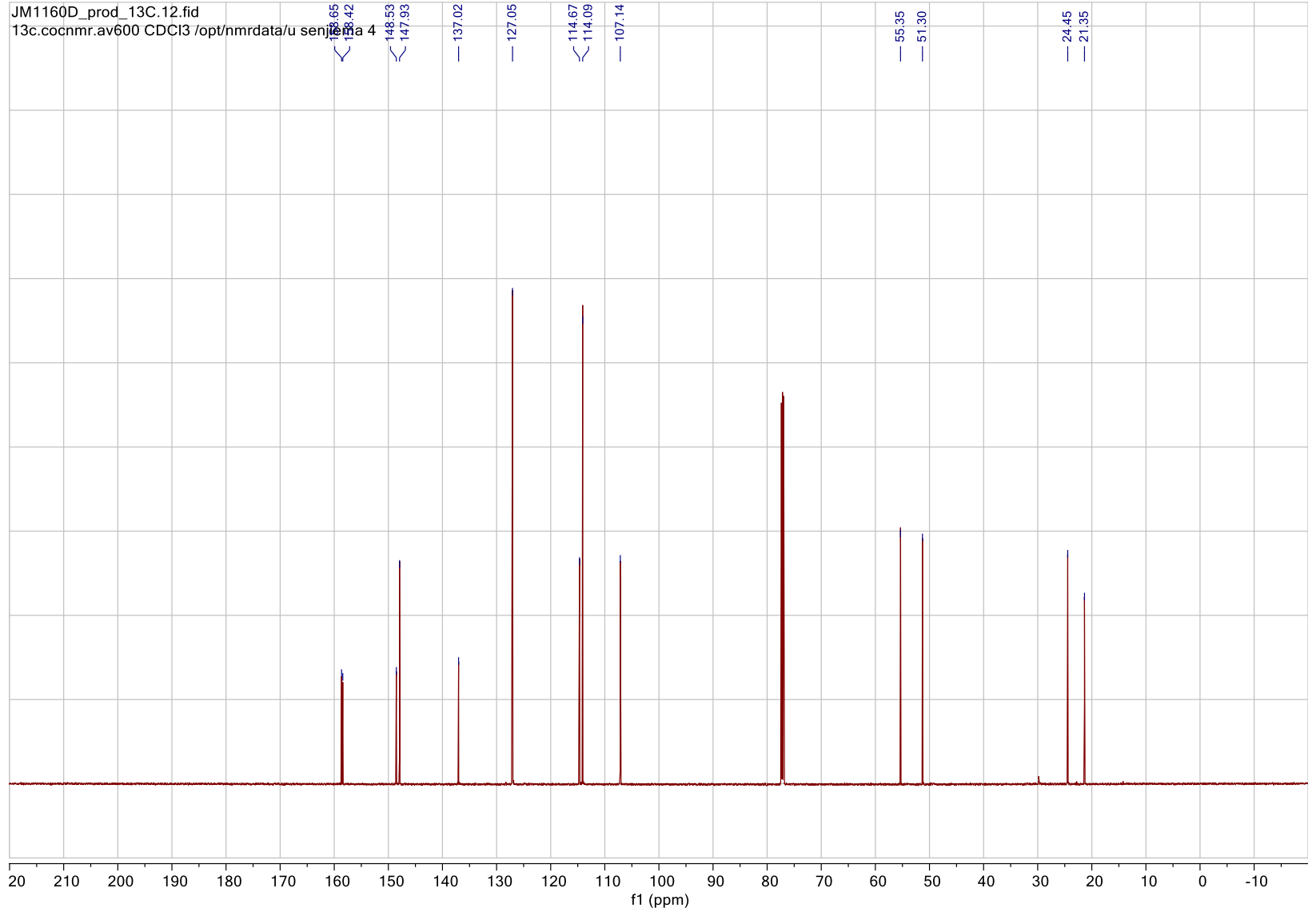
<smiles>Cc1ccnc(NC(C)c2ccc(C(F)(F)F)cc2)c1</smiles>

4-methyl-N-(1-(4-(trifluoromethyl)phenyl)ethyl)pyridin-2-amine (22a)

\section{${ }^{1} \mathbf{H}$}

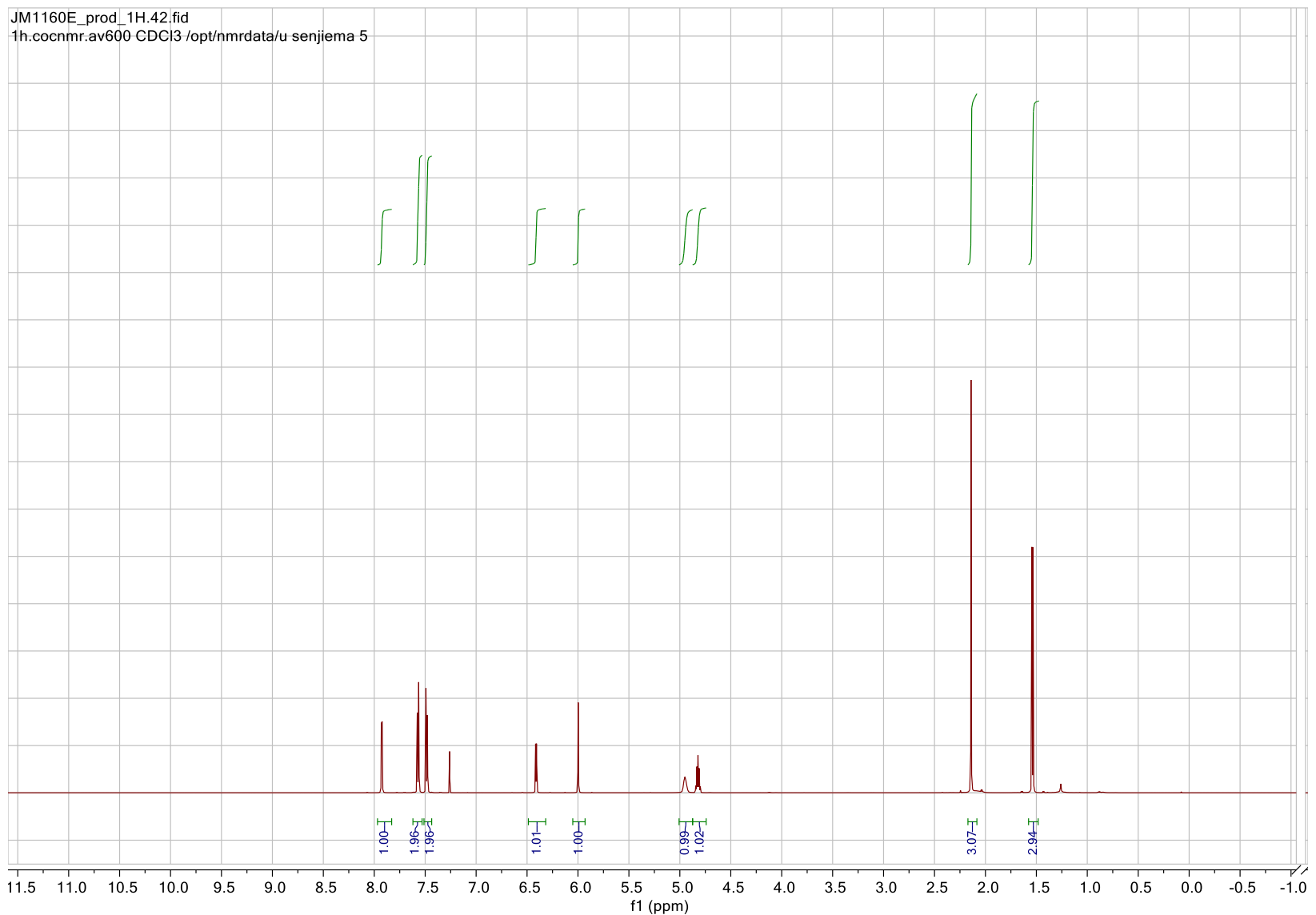


${ }^{13} \mathrm{C}$

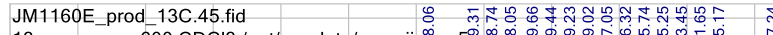

13c.cocnmr.av600 CDCl3/opt/nmrdata/u senjilienha

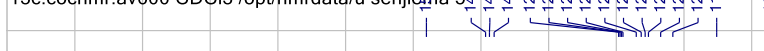
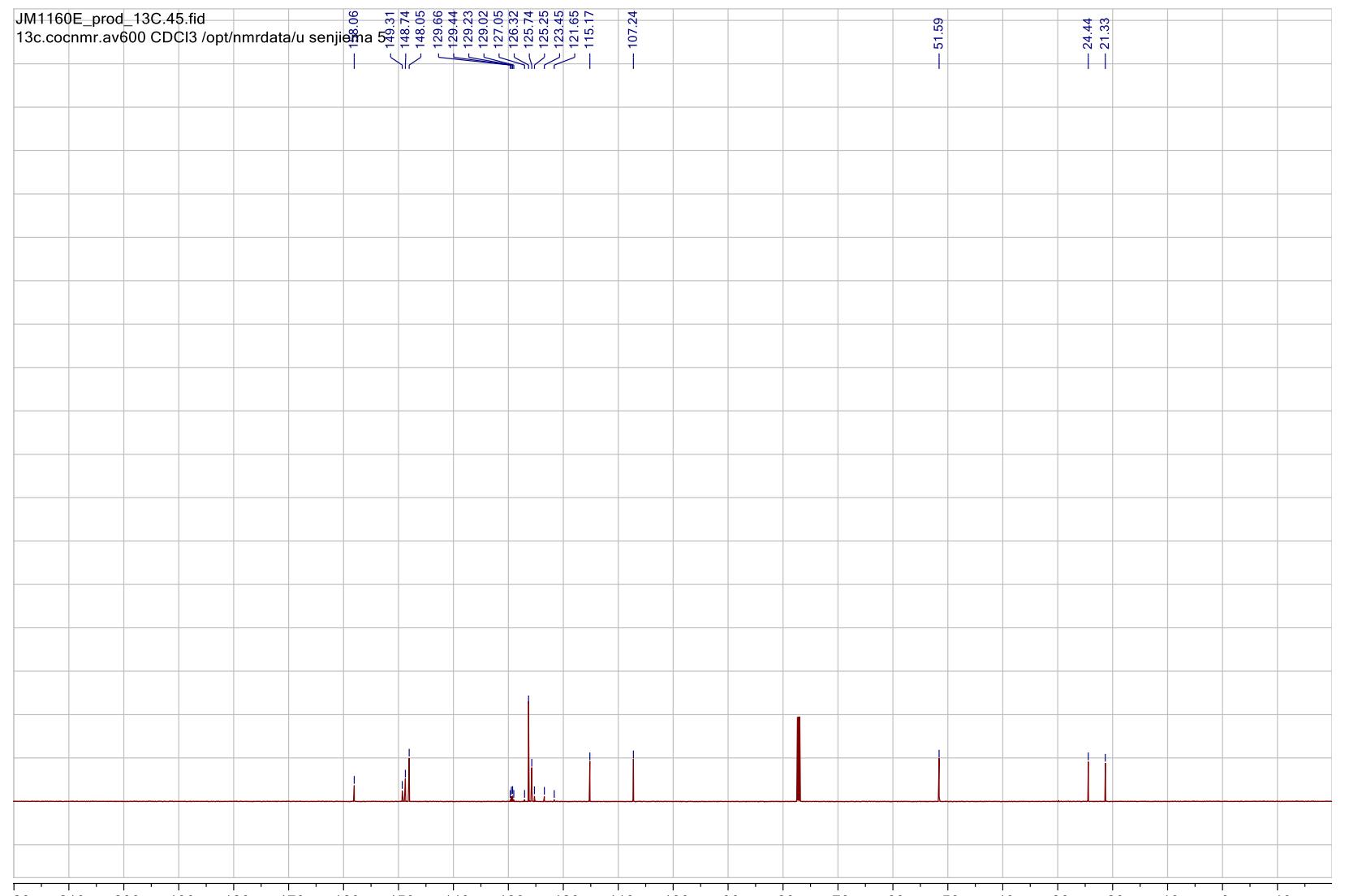

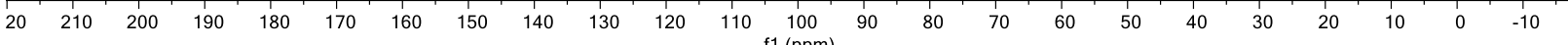




\section{${ }^{19} \mathbf{F}$}

JM1160E_prod_19F.43.fid

19f.cocnmr.av600 CDCl3 /opt/nmrdata/u senjiema 5
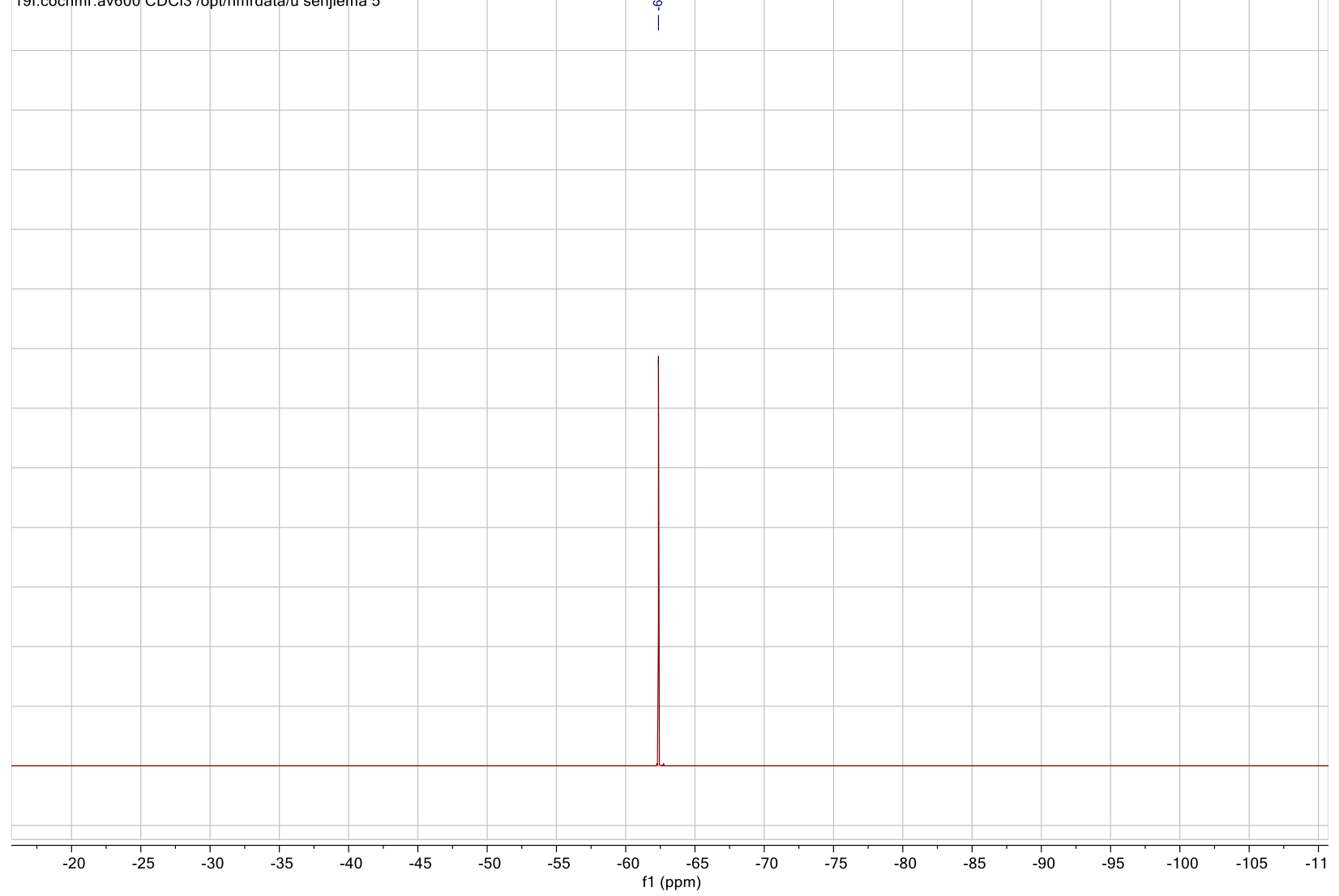

S111 
<smiles>Cc1ccnc(NC(C)c2ccc(Cl)cc2)c1</smiles>

N-(1-(4-chlorophenyl)ethyl)-4-methylpyridin-2-amine (23a)

\section{${ }^{1} \mathbf{H}$}

JM1160F_prod_1H.15.fid

1h.cocnmr.av600 CDCl3 /opt/nmrdata/u senjiema 6

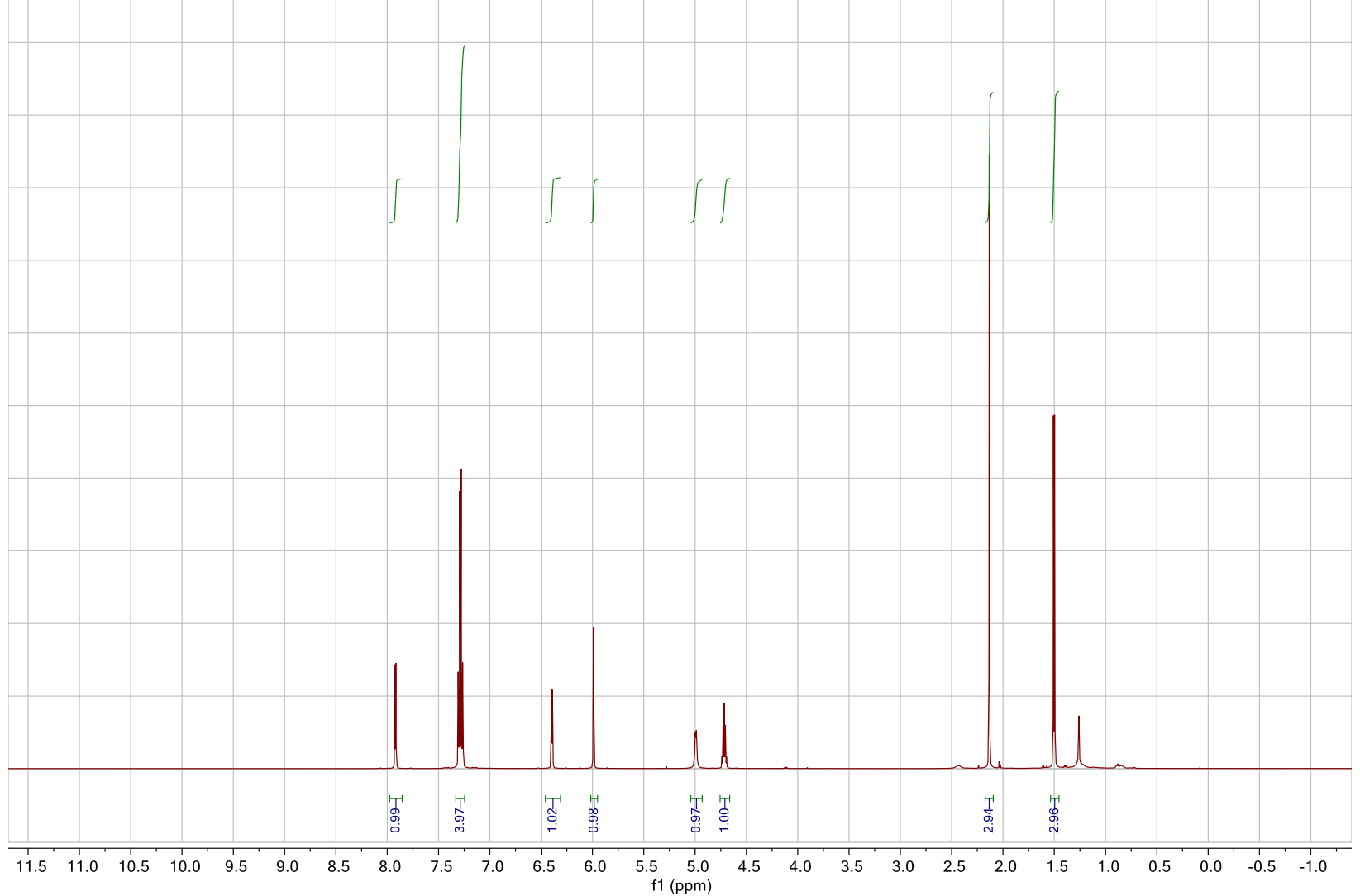


${ }^{13} \mathrm{C}$

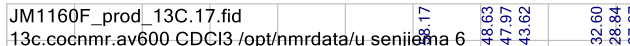

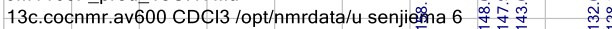

(i)

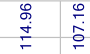

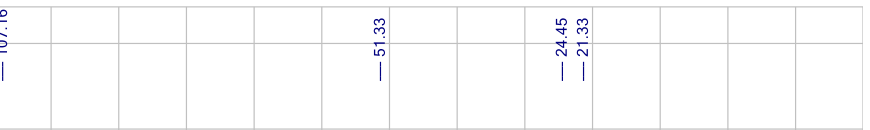

\begin{tabular}{|l|l|l|l|l|l|l|l|l|l|l|l|l|l|l|l|l|l|l|l|}
\hline & & & & & & & & & & & & & & & & & & & \\
\hline
\end{tabular}
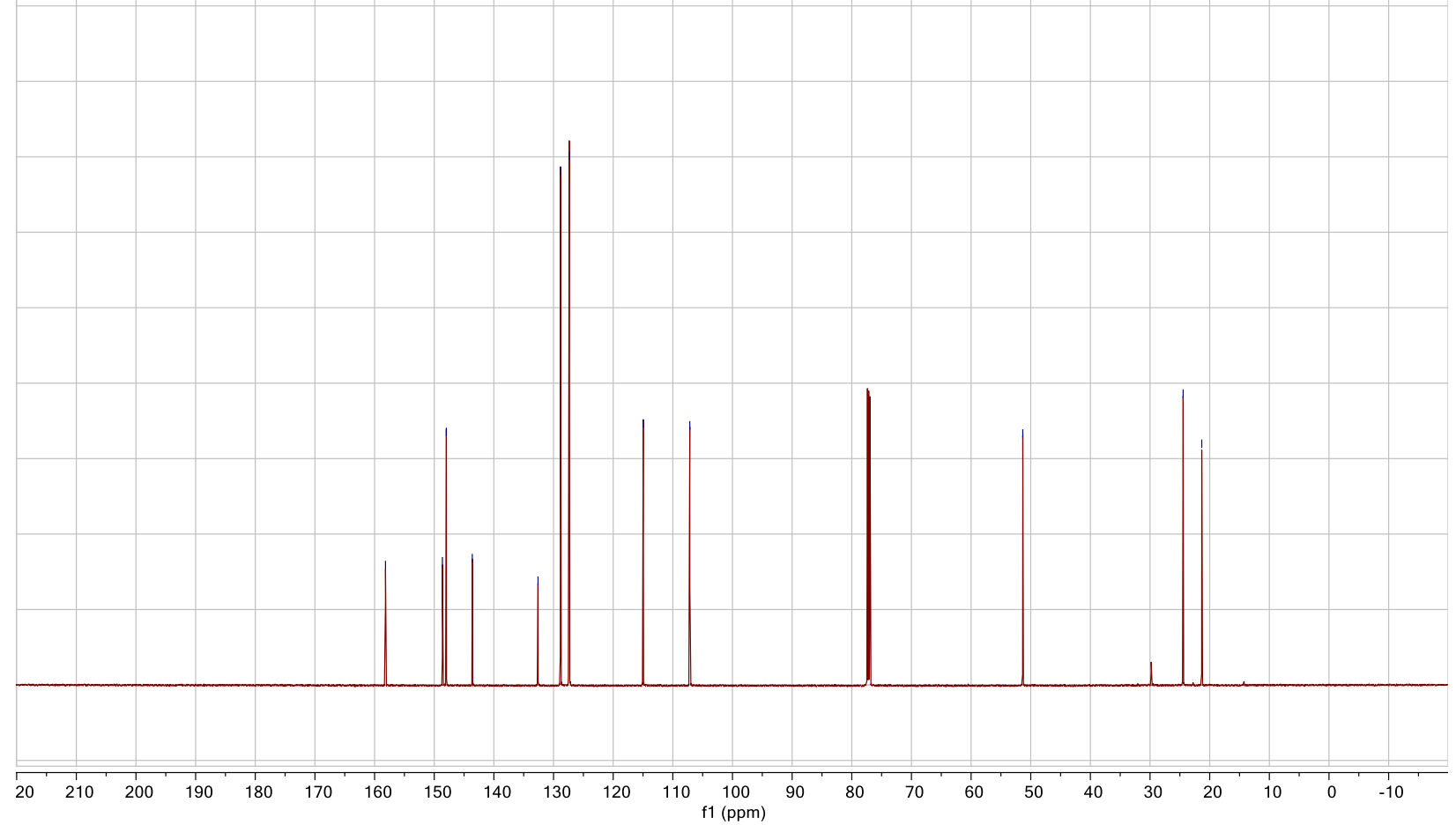
<smiles>Cc1ccnc(NC(C)c2ccc(Br)cc2)c1</smiles>

N-(1-(4-bromophenyl)ethyl)-4-methylpyridin-2-amine (24a)

\section{${ }^{1} \mathbf{H}$}

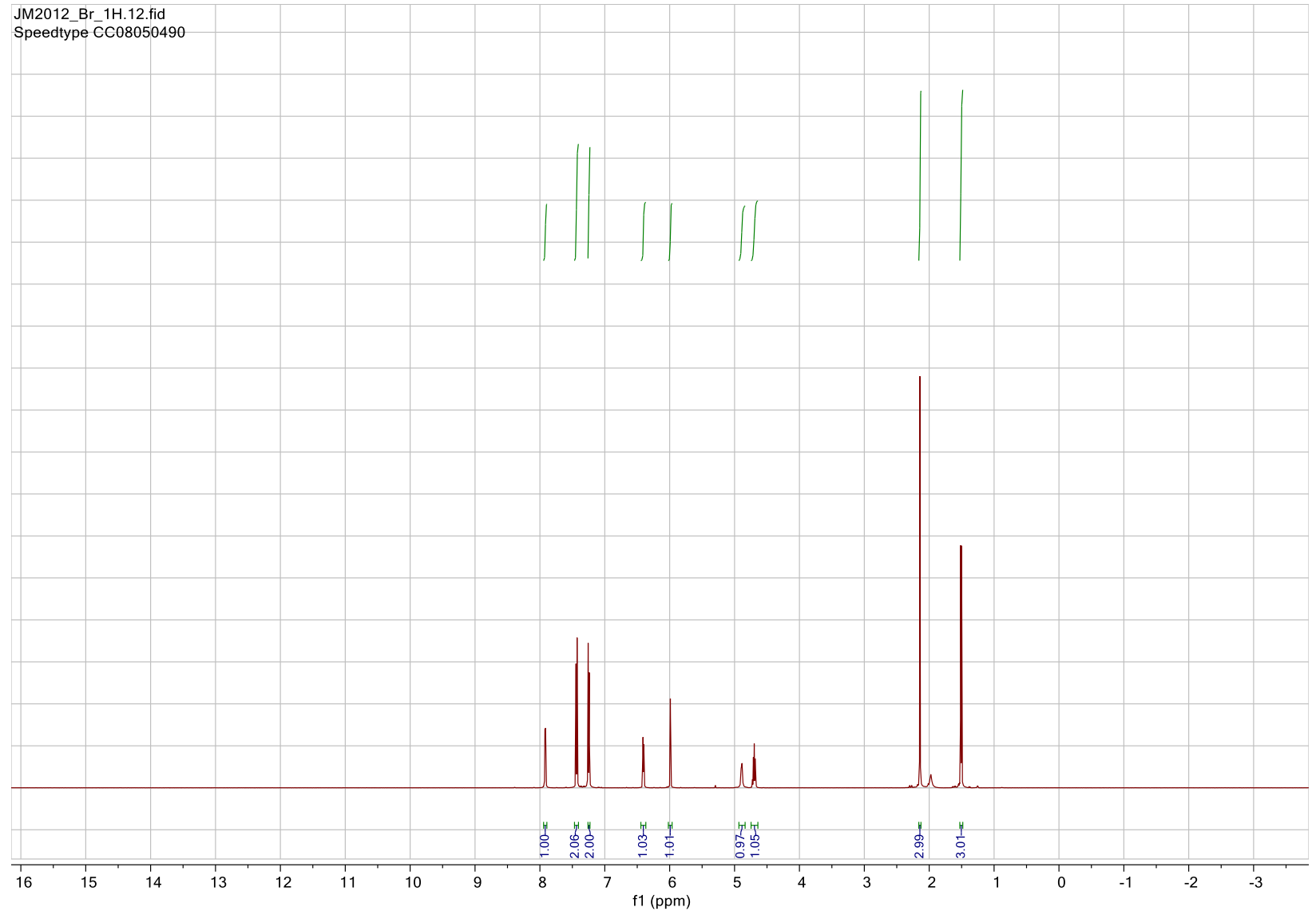


${ }^{13} \mathrm{C}$

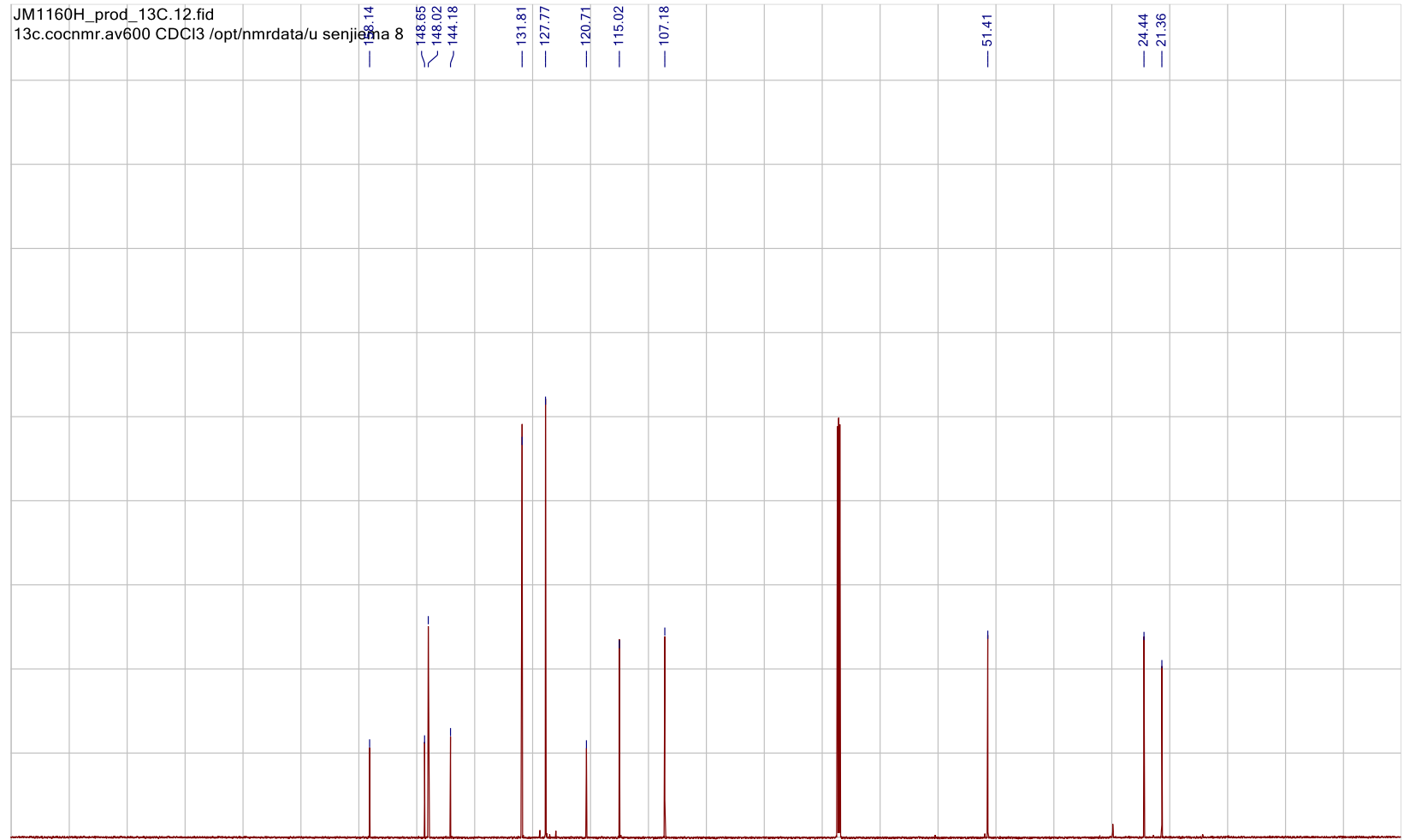

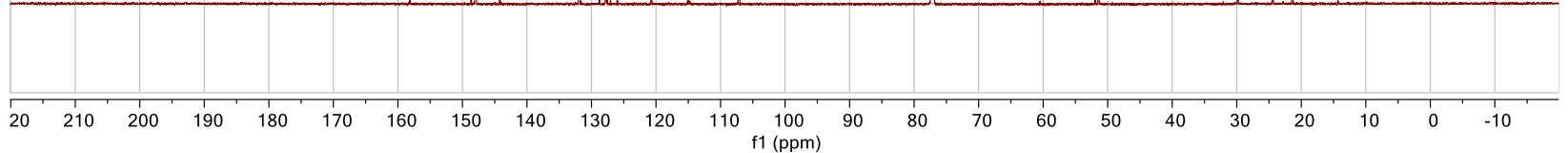




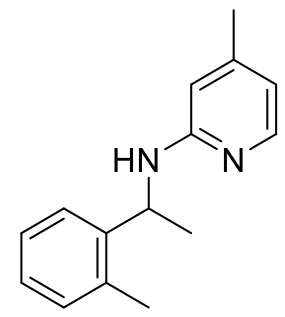

4-methyl-N-(1-(o-tolyl)ethyl)pyridin-2-amine (25a)

\section{${ }^{1} \mathbf{H}$}

JM1160G_prod_1H.10.fid

1h.cocnmr.ave0

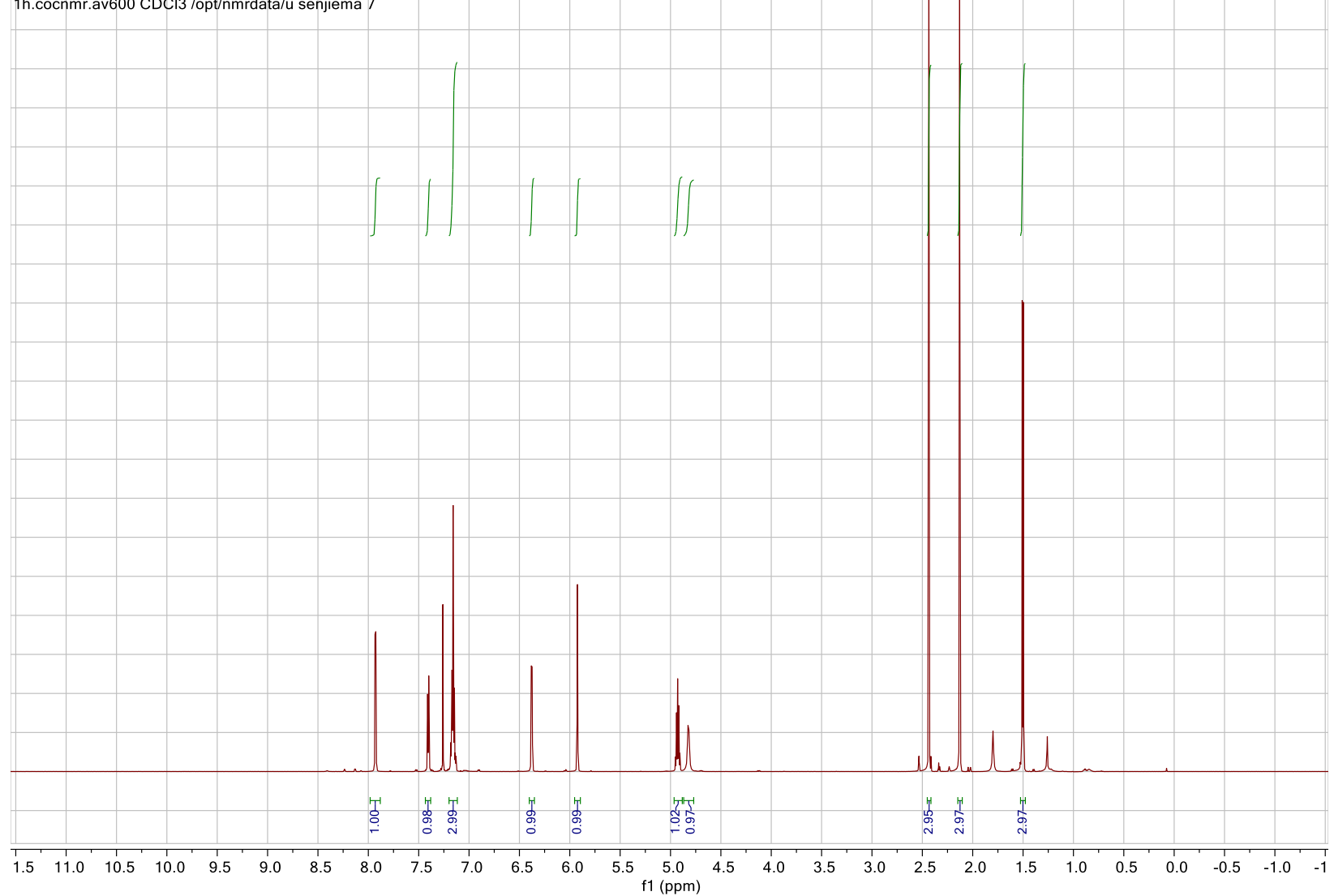

${ }^{13} \mathrm{C}$ 


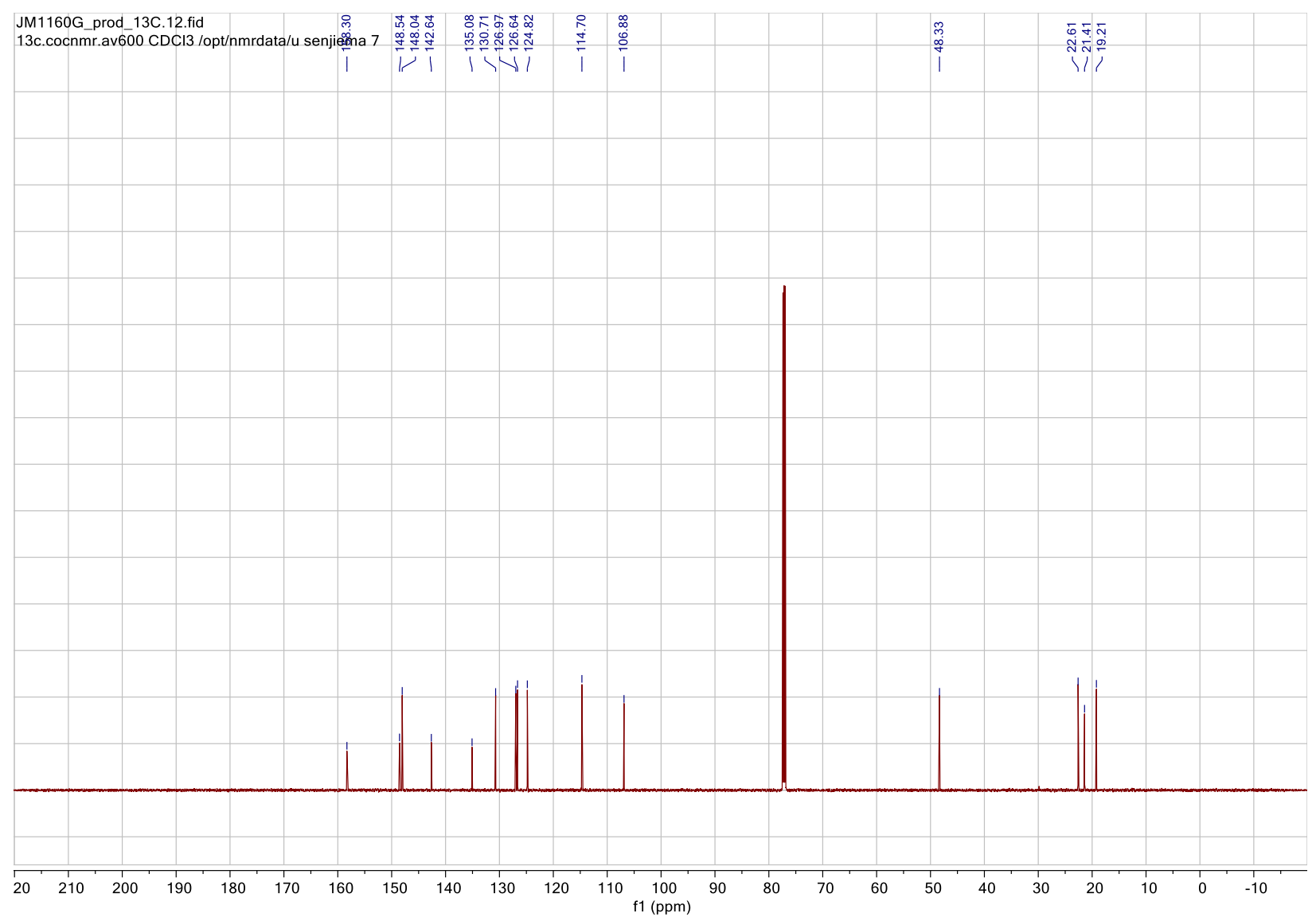




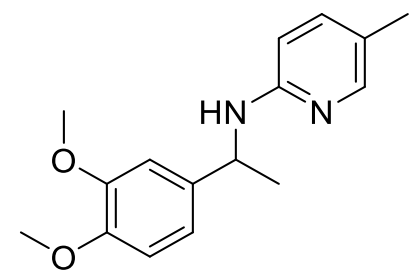

$N$-(1-(3,4-dimethoxyphenyl)ethyl)-5-methylpyridine-2-amine (26a)

${ }^{1} \mathbf{H}$

co-152-prod1h600.1.tid

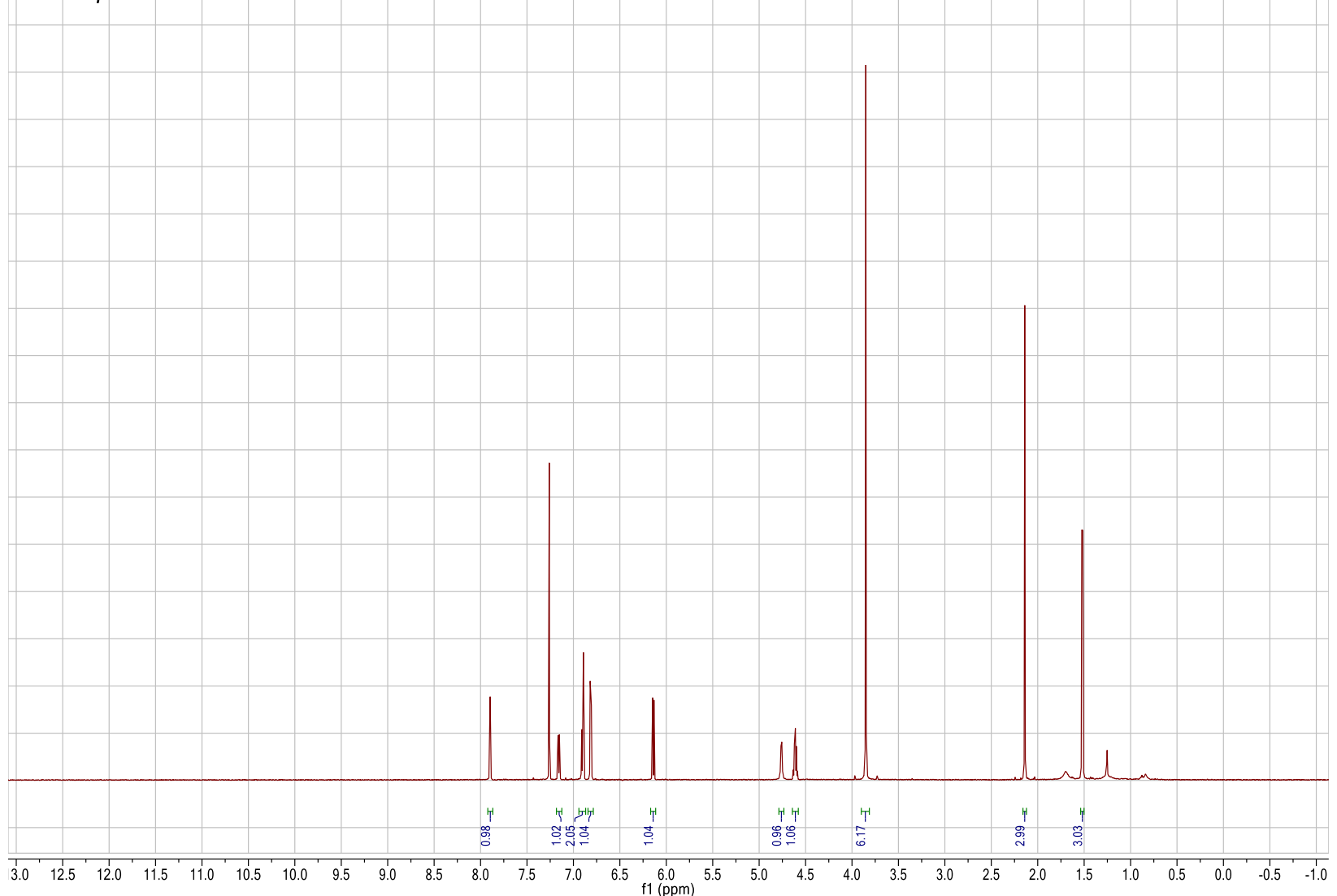


${ }^{13} \mathrm{C}$

co-152-prod13c600.1.tid

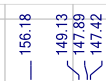

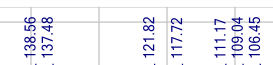

1 पर

i)

1111

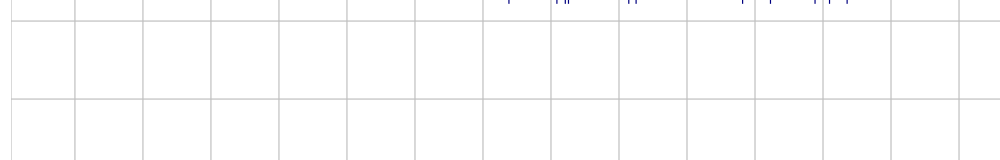
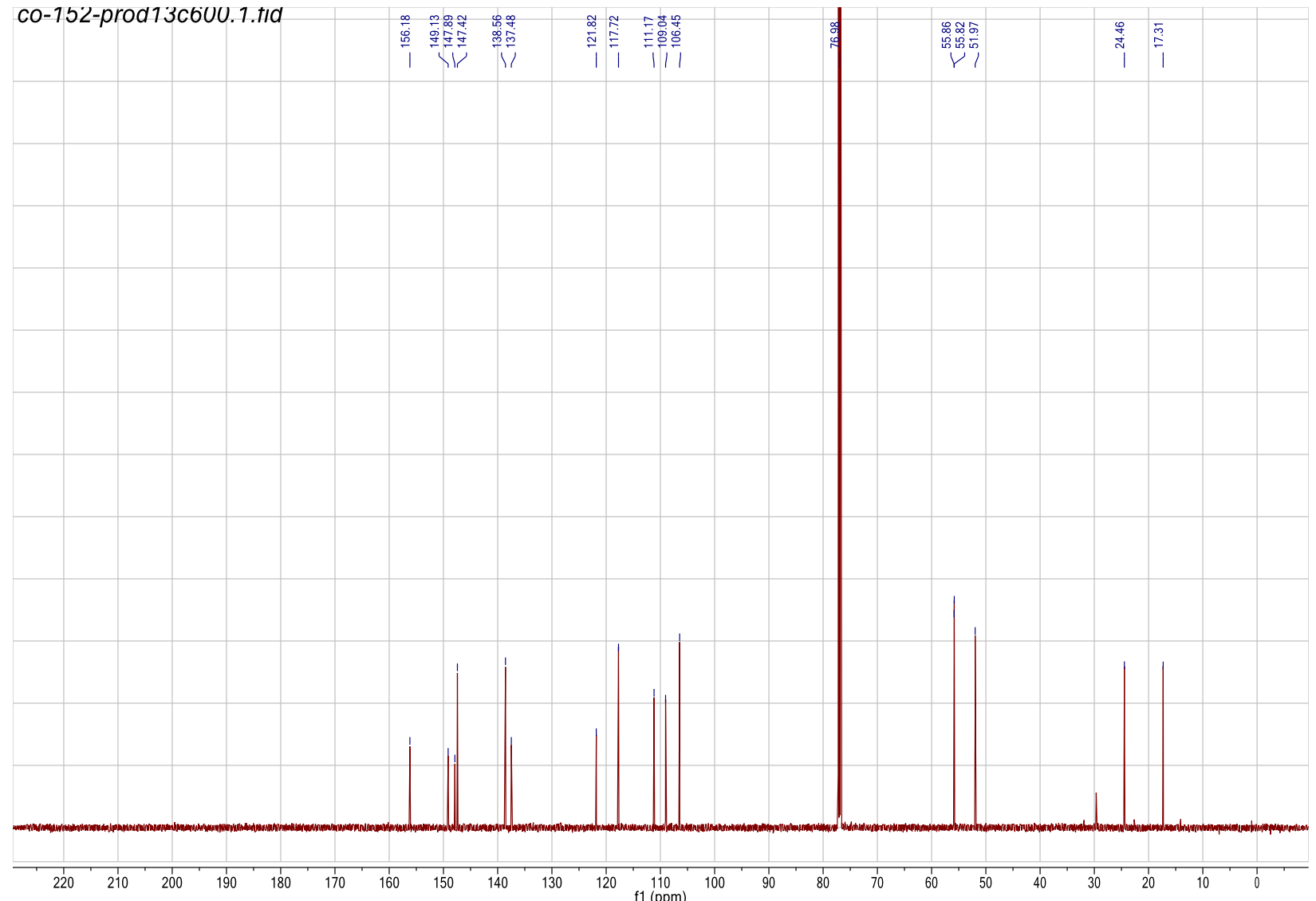

$20 \underset{110}{110} 100$
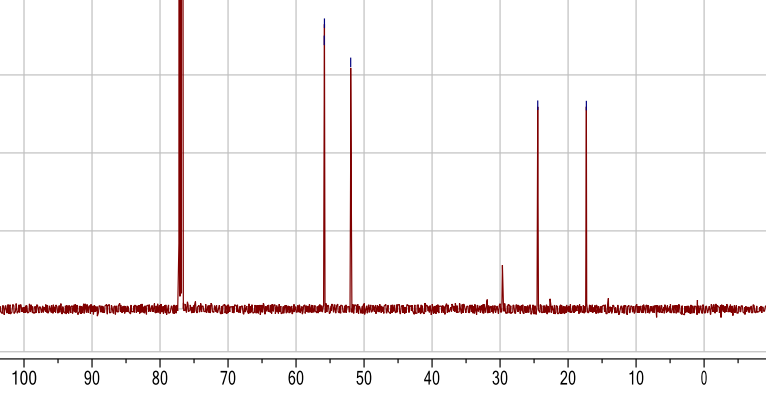
<smiles>Cc1ccc(NC(C)c2ccc3ccccc3c2)nc1</smiles>

$\mathrm{N}$-(ethyl-naphthalene-2-yl)-5-methylpyridin-2-amine (27a) ${ }^{1} \mathbf{H}$

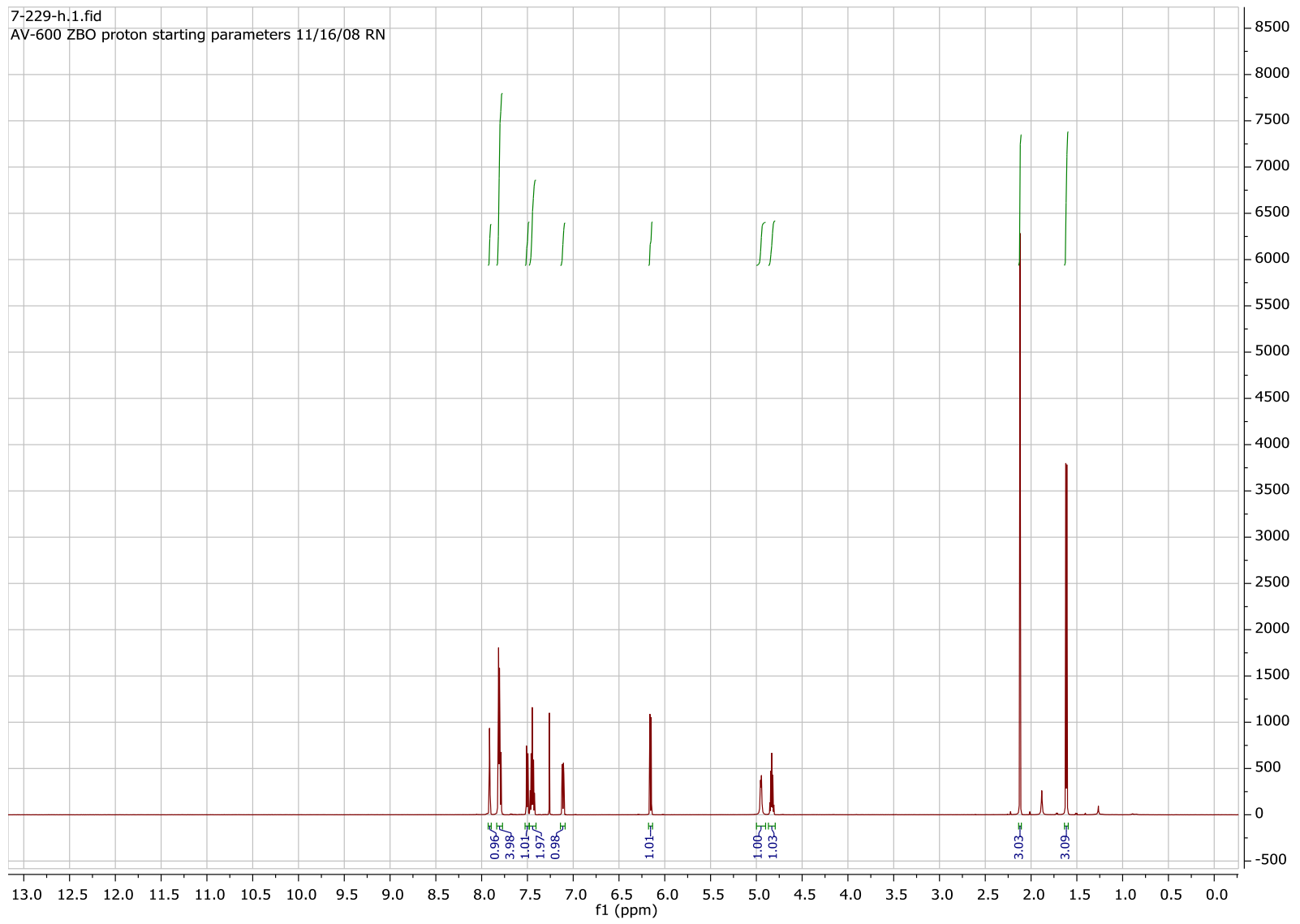


${ }^{13} \mathrm{C}$

7-229-c.1.fid

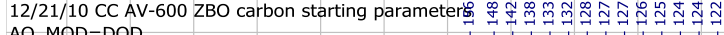

AQ_MOD=DQD

年,
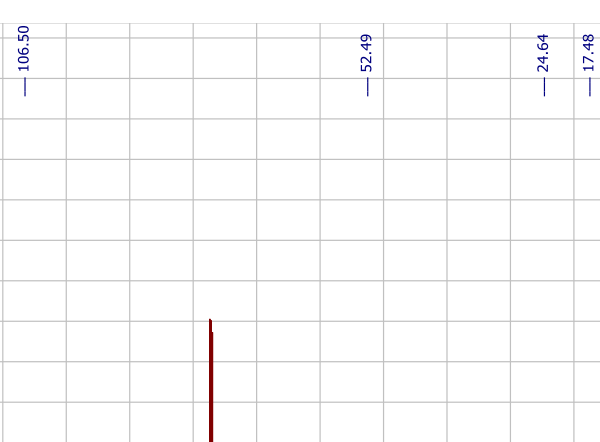

25000

$-24000$

$-23000$

$-22000$

21000

20000

19000

$-18000$

$-17000$

$-16000$

$-15000$

$-14000$

$-13000$

12000

$-11000$

$-10000$

$-9000$

$-8000$

$-7000$

6000

5000

$-4000$

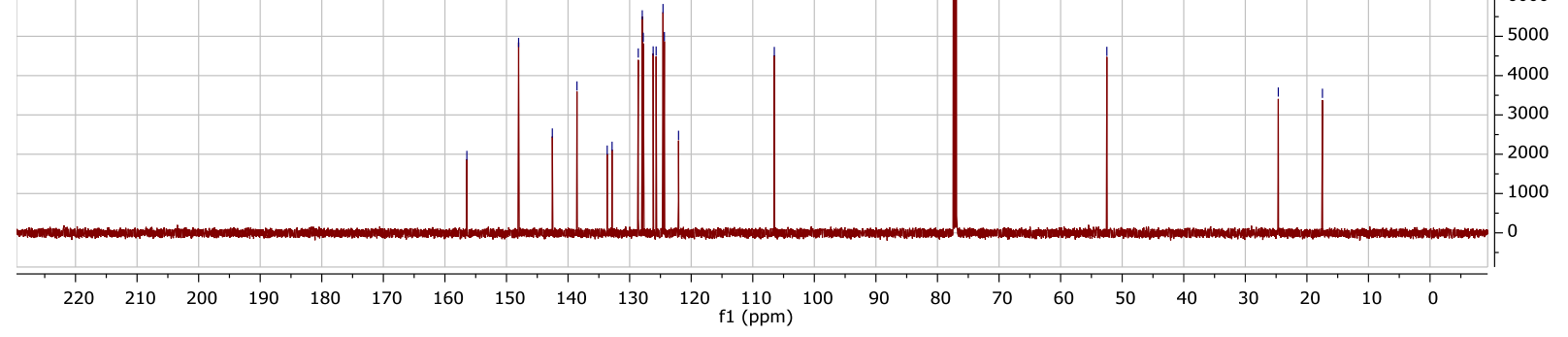




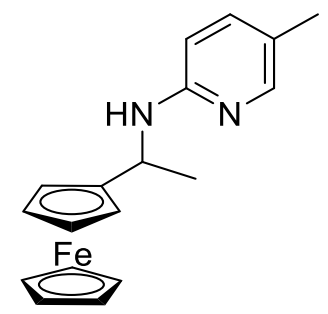

$\mathrm{N}$-(ethylferrocene-2-yl)-5-methylpyridin-2-amine (28a)

${ }^{1} \mathbf{H}$

$7-205.1$ fid

AV-600 ZBO proton starting parameters $11 / 16 / 08 \mathrm{RN}$

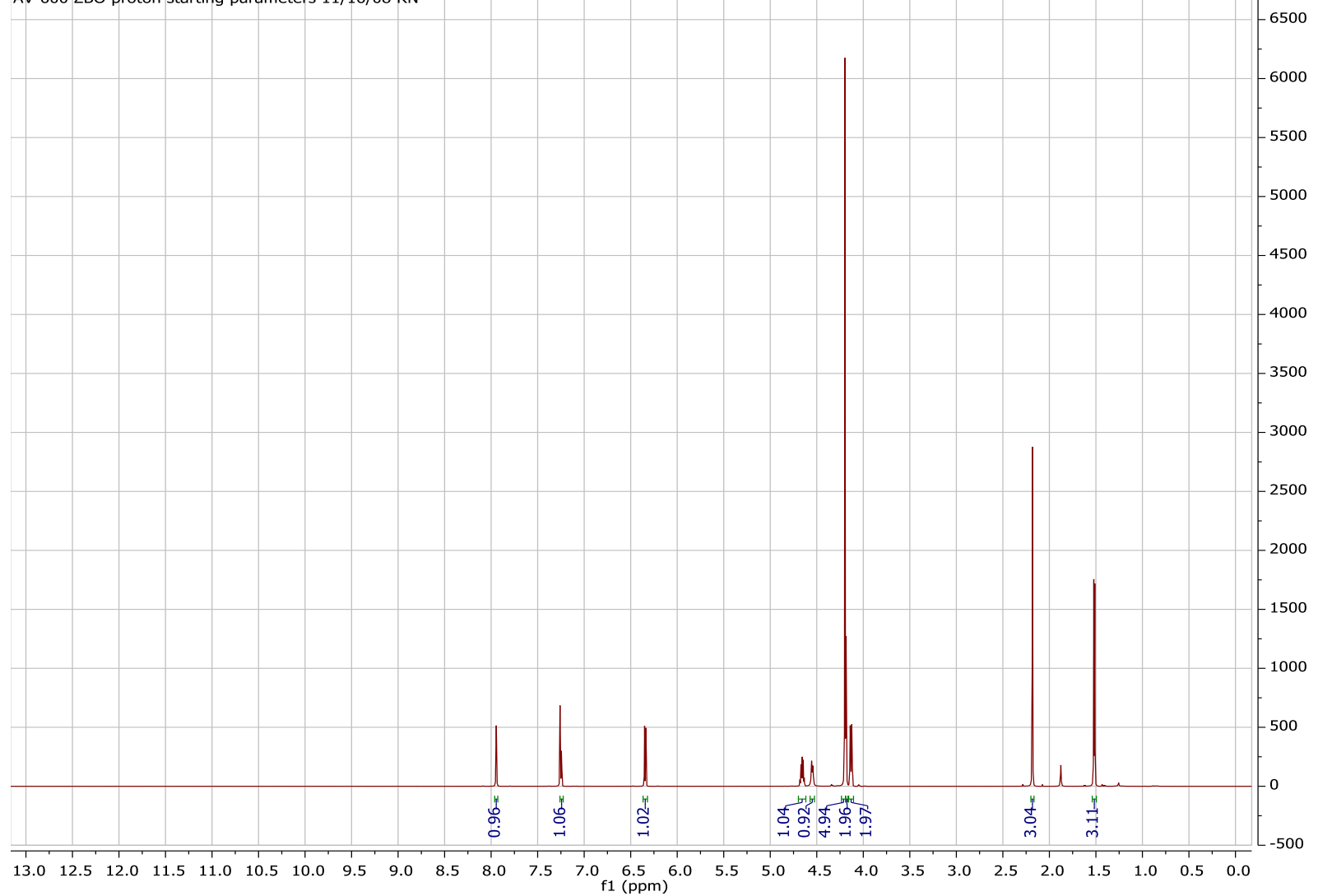


${ }^{13} \mathrm{C}$

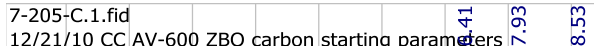

$12 / 21 / 10$ CC AV

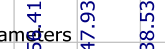

AQ_MOD $=D Q D$

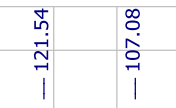

$\stackrel{\substack{\infty \\ m \\ n}}{a}$

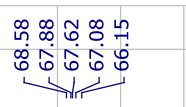

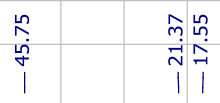

160000

150000

140000

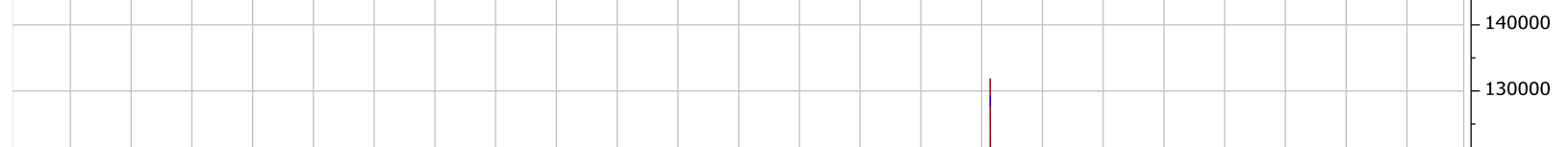

\begin{tabular}{l|l|l|l|l|l|l|l|l|l|l|l|l|l|l|} 
& \\
\hline
\end{tabular}
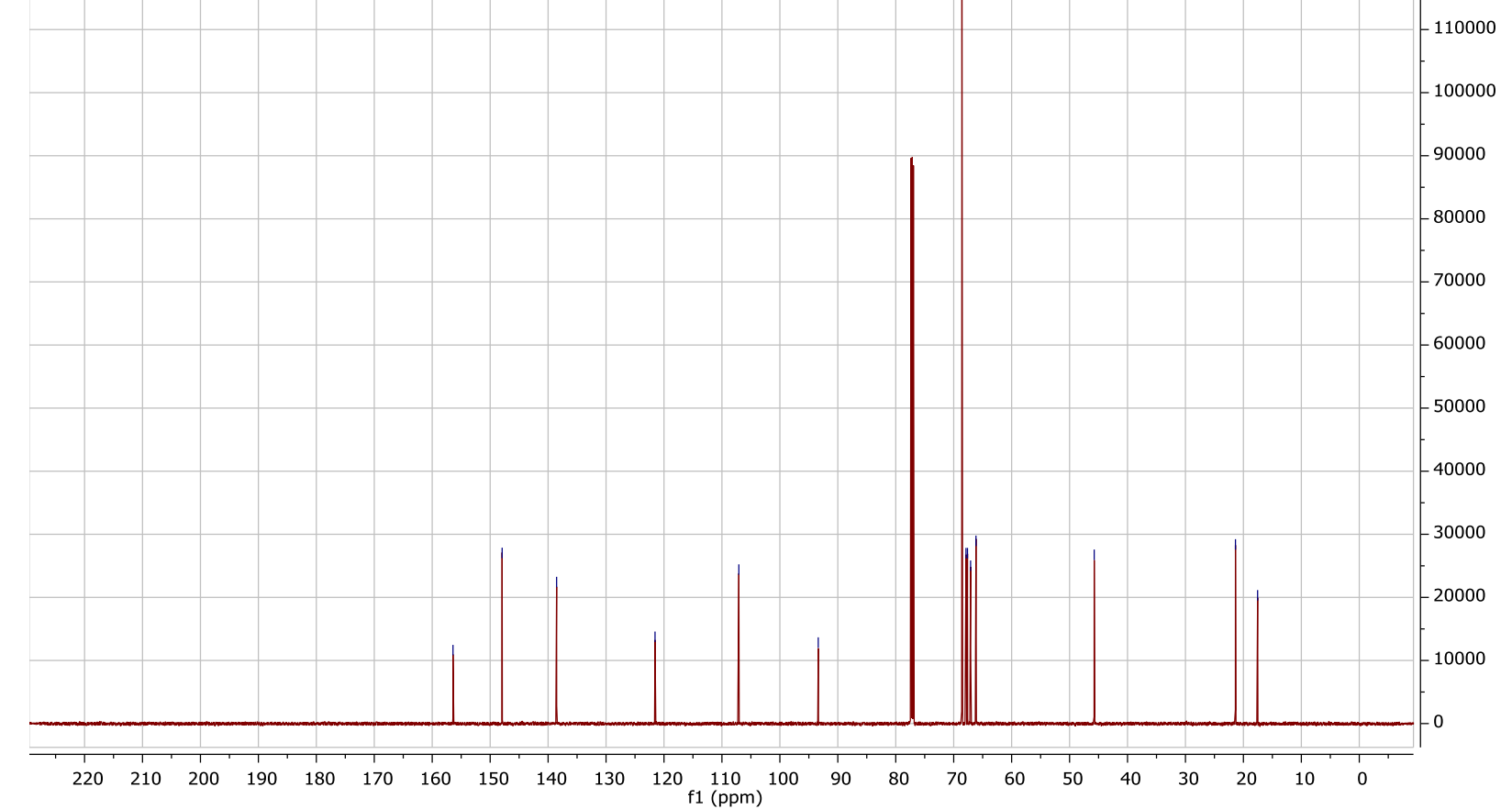
$\overbrace{5}^{N H B o c}$

tert-butyl octan-2-ylcarbamate (2c)

${ }^{1} \mathbf{H}$

JM2053A_1H.1.fid
$\begin{aligned} & \text { 1H starting parameters - HC 06/17/2019 } \\ & \text { No decoupling }\end{aligned}$

No decoupling
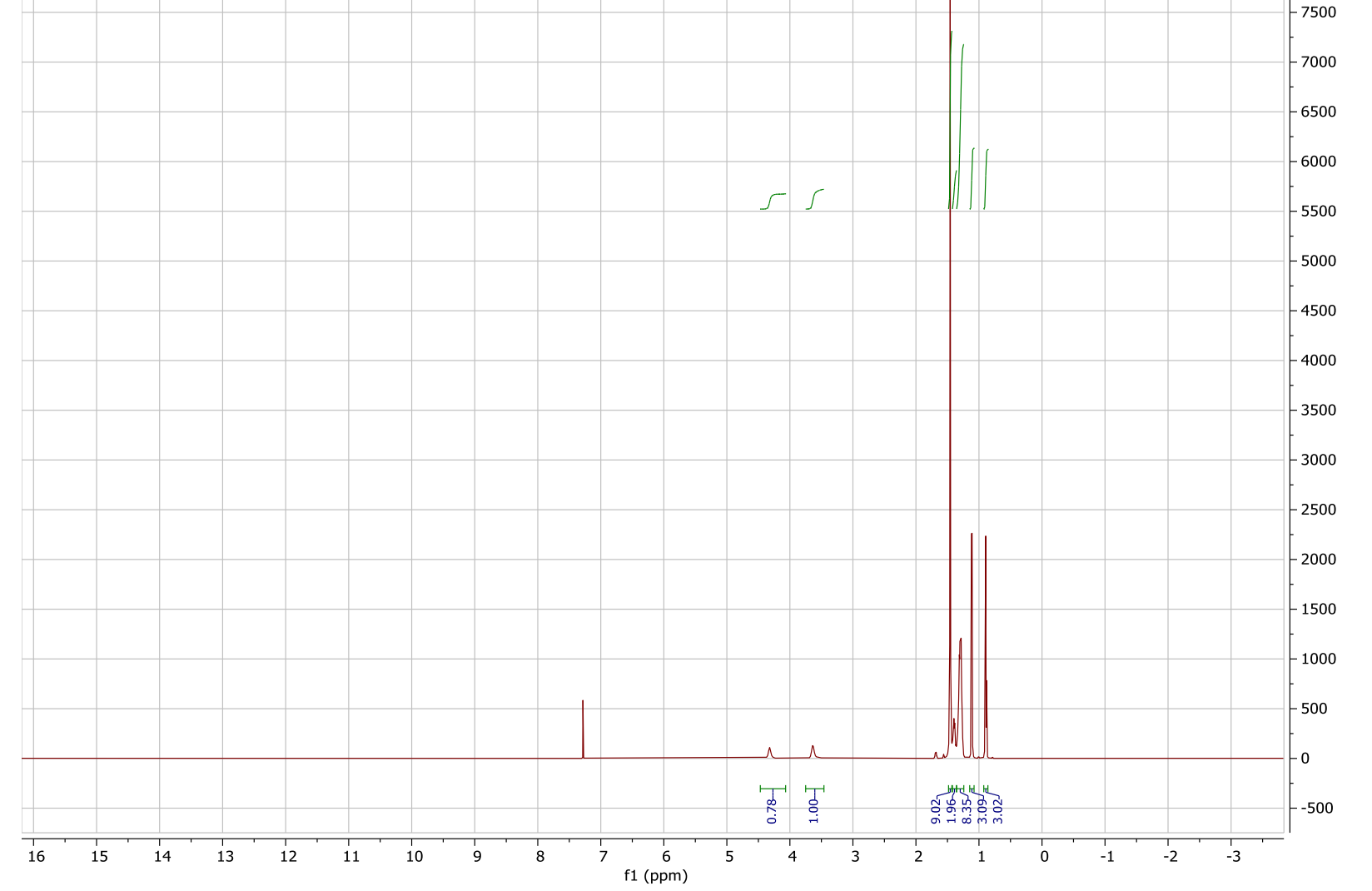


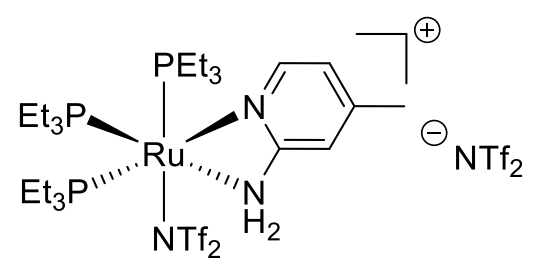

Ru(PEt $)_{3}$ (amine)(NTf $)_{2}$ (29)

\section{${ }^{1} \mathbf{H}$}

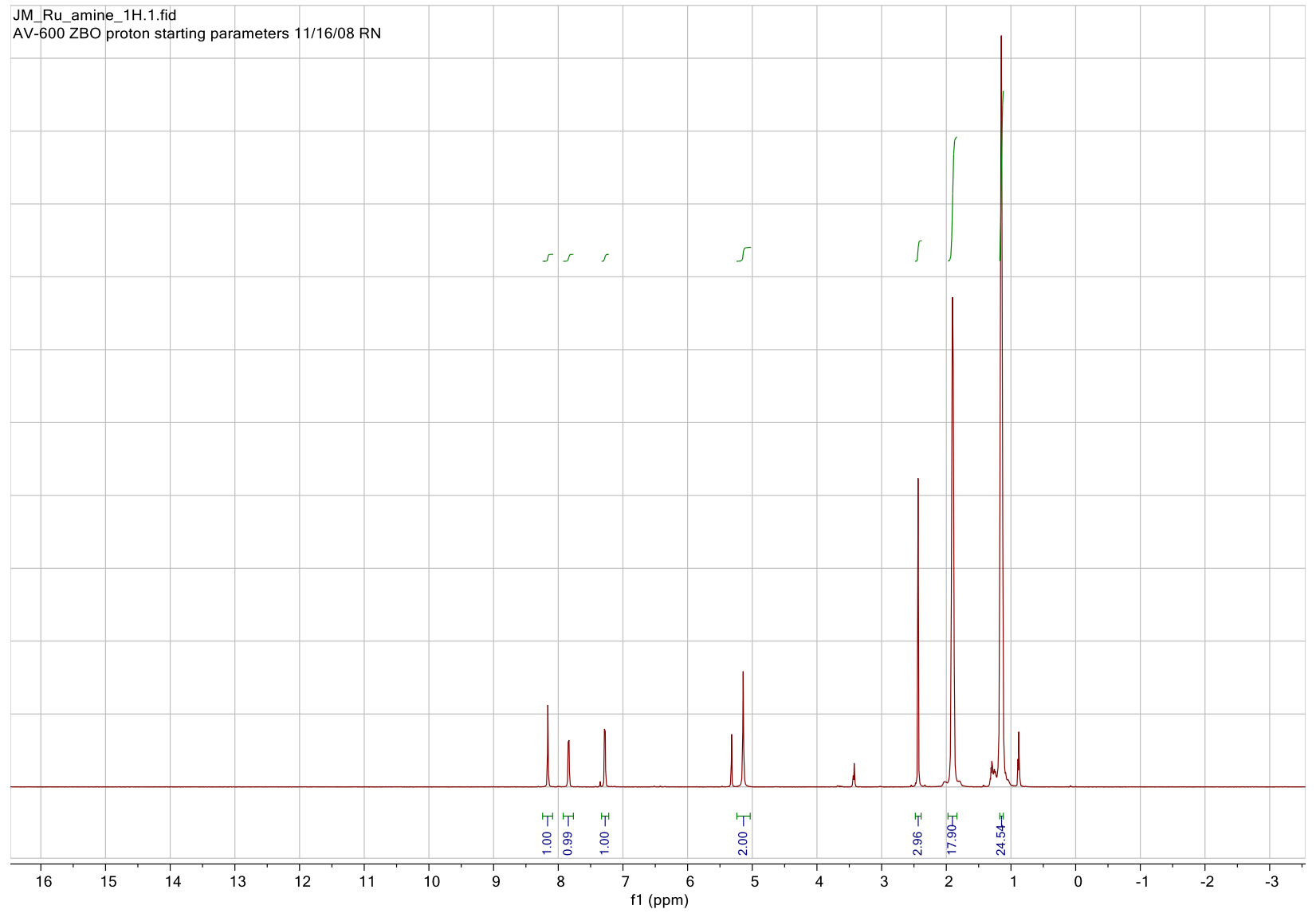




\section{$31 \mathbf{P}$}

JM_Ru_amine_31P.1.fid
AVQ-400 QNP 31P Starting parameters. Trimethyl phosphate=3.0 ppm. 7/16/03 Rev 7/22\% $\stackrel{m}{3}^{m}$ RN

AVQ-400 QNP 31P Starting parameters. Trimethyl phosphate $=3.0 \mathrm{ppm} .7 / 16 / 03$ Rev 7/228 3 RN

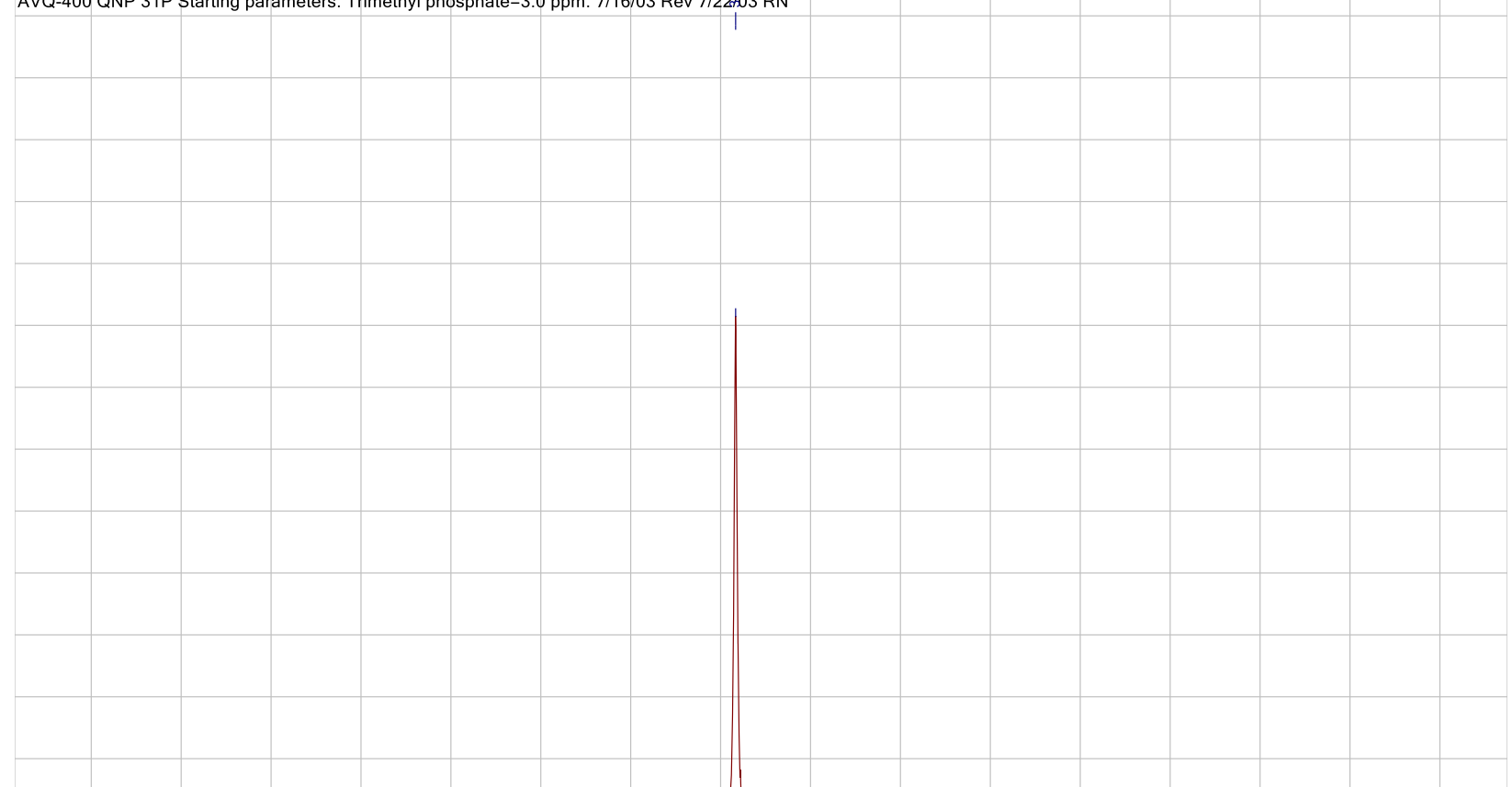

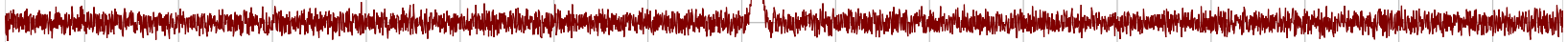

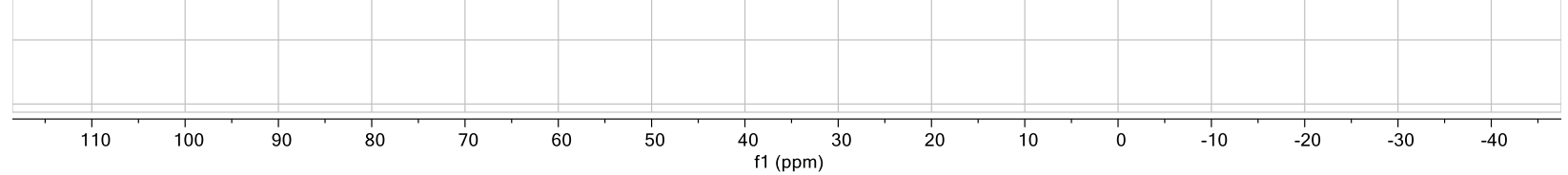




\section{${ }^{19} \mathrm{~F}$}

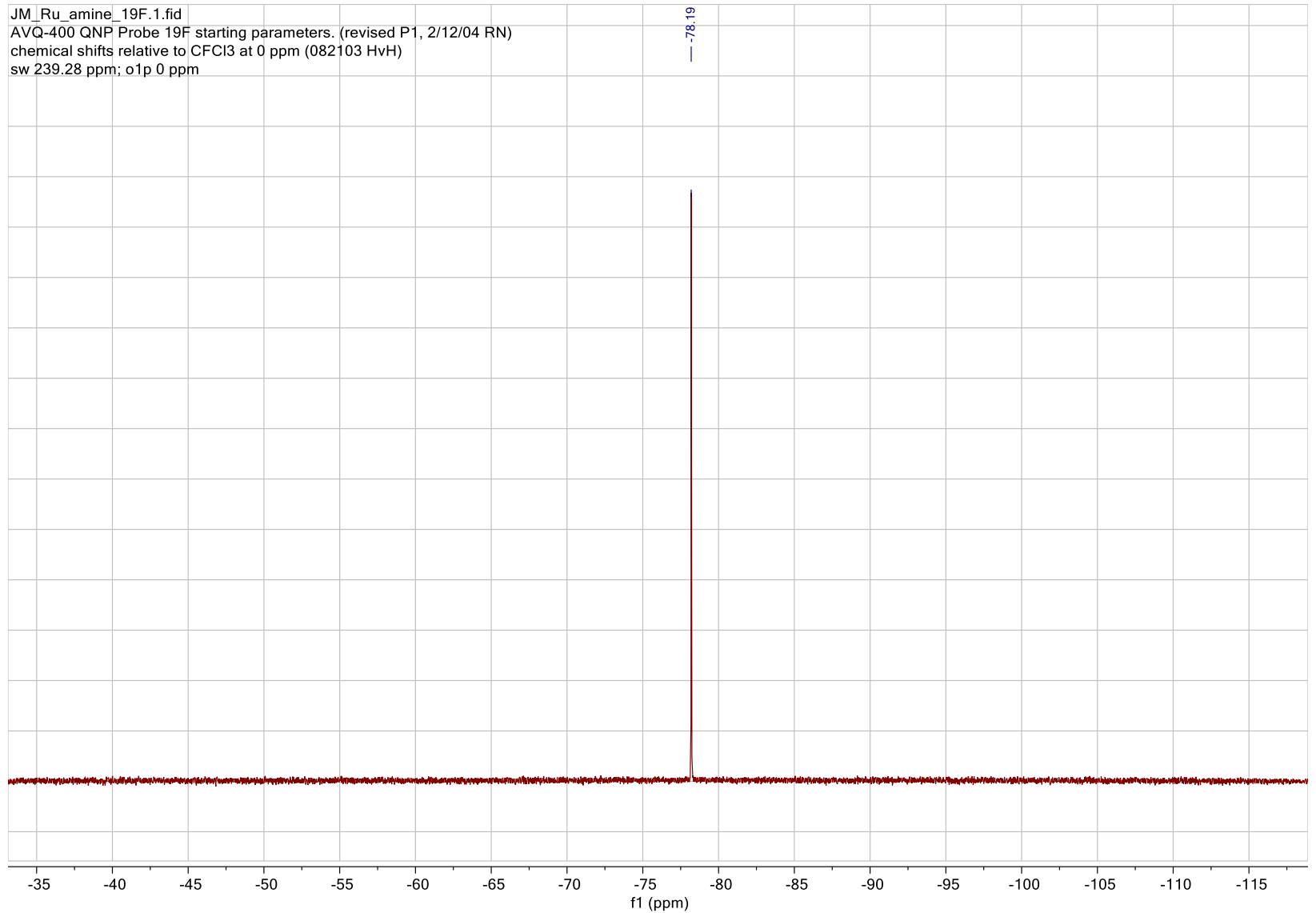




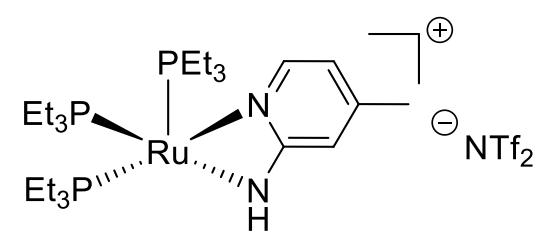

\section{Ru(PEt $)_{3}$ (amide)(NTf 2 ) (30)}

\section{${ }^{1} \mathbf{H}$}

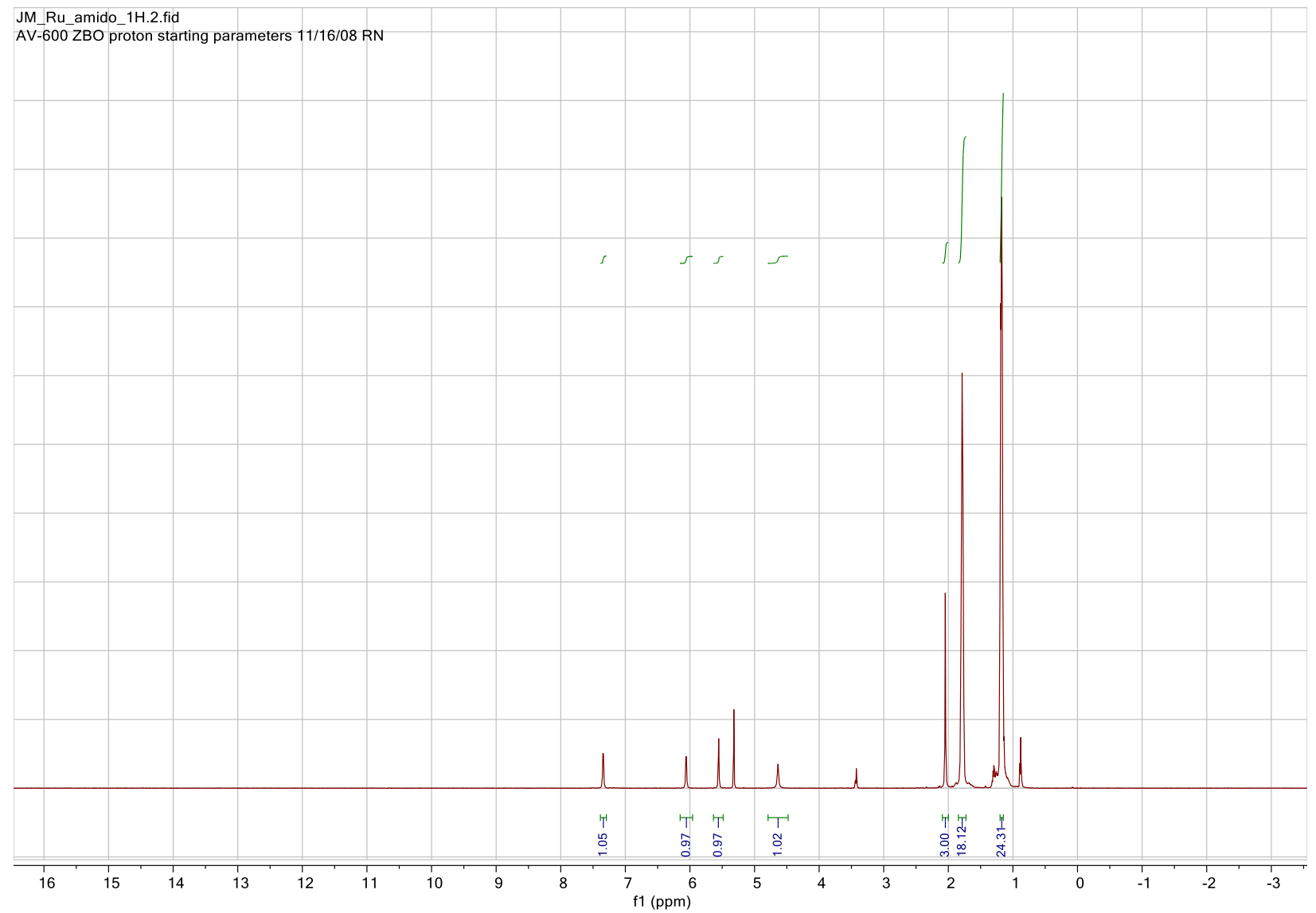




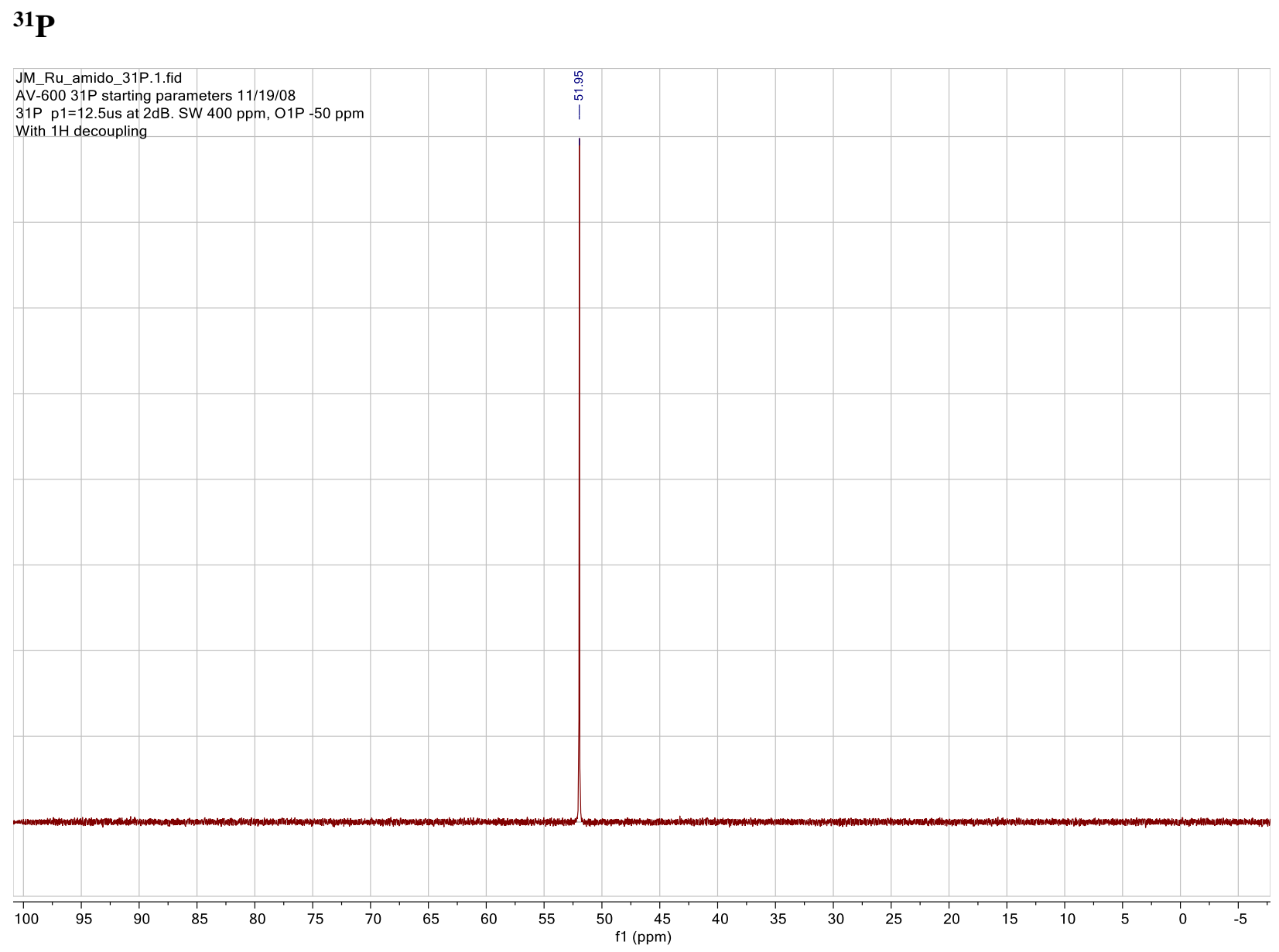




\section{${ }^{19} \mathrm{~F}$}

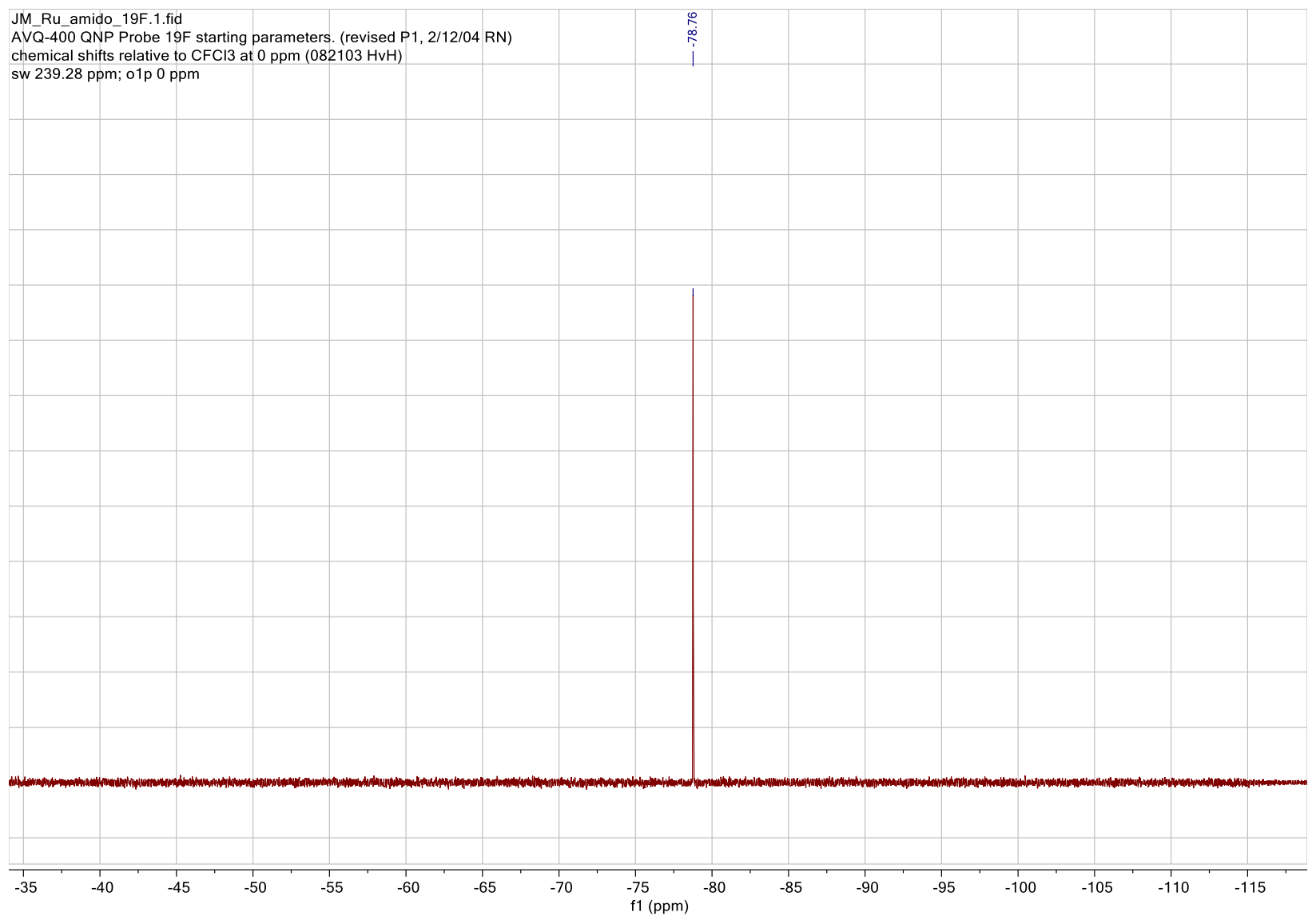


<smiles>CCCCCCCC</smiles>

\section{$\operatorname{Ru}\left(\mathrm{H}_{)}\left(\mathrm{H}_{2}\right)\left(\mathrm{PEt}_{3}\right)_{3}(\right.$ amine $)\left(\mathrm{NTf}_{2}\right)(43)$}

\section{${ }^{1} \mathbf{H}$}

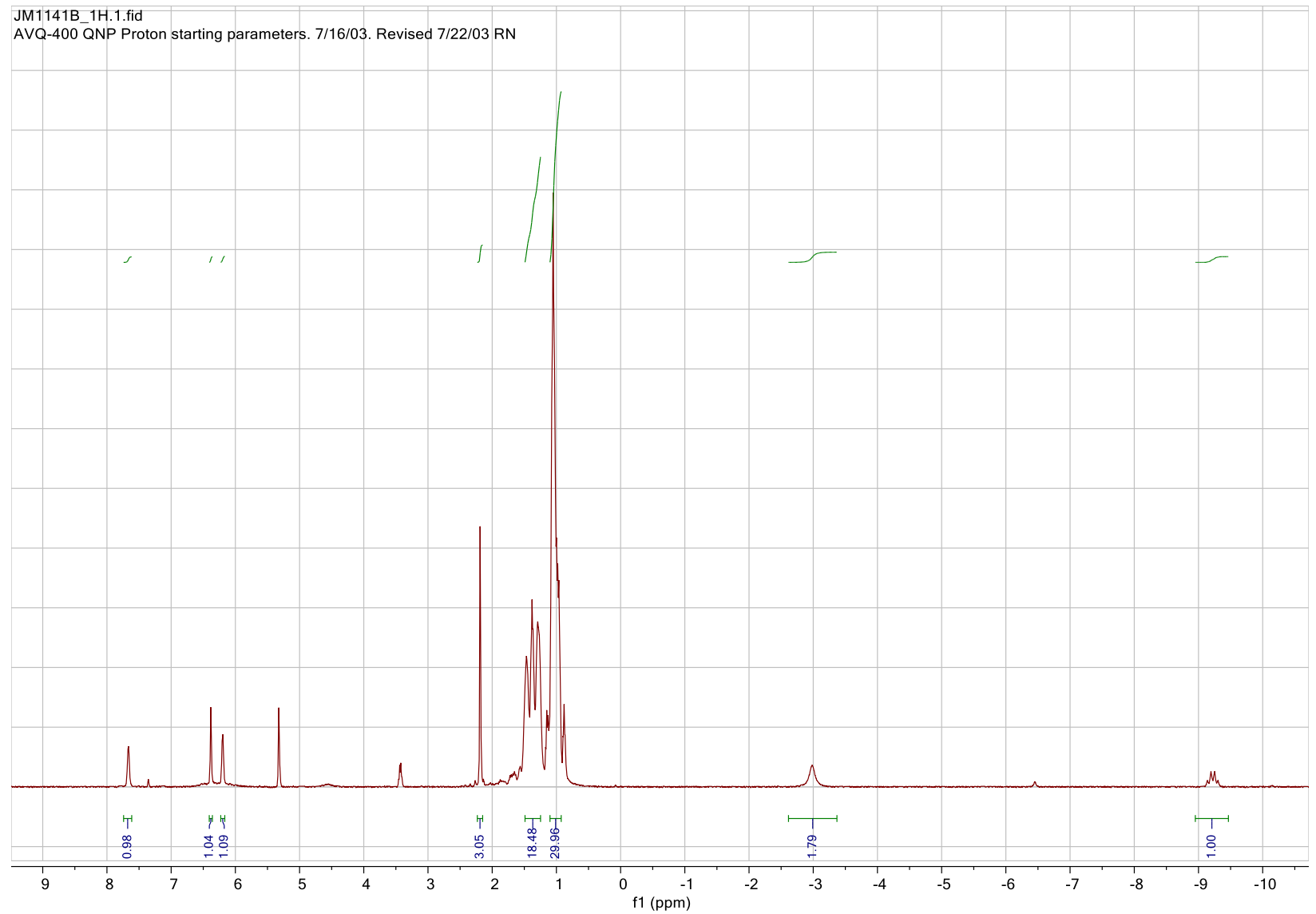




\section{$31 \mathbf{P}$}

JM1141C_31P.1.fid
AVQ-400 QNP 31P Starting parameters. Trimethyl phosphate=3.0 ppm. 7/16/03 Rev 7/22/03 RN AVQ-400 QNP 31P Starting parameters. Trimethyl phosphate $=3.0 \mathrm{ppm} .7 / 16 / 03 \mathrm{Rev} 7 / 22 / 03 \mathrm{RN}$
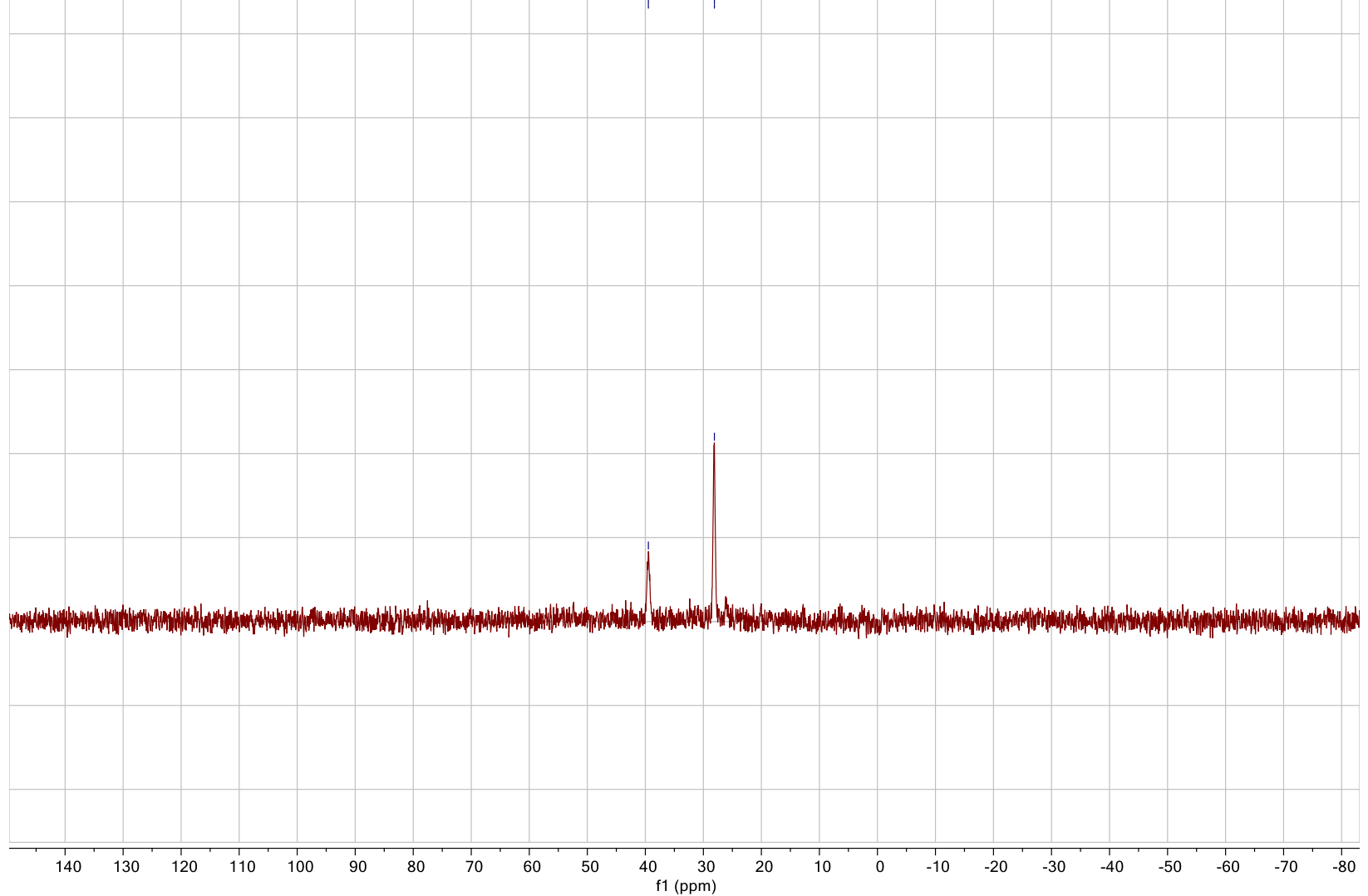